
NUNC COGNOSCO EX PARTE

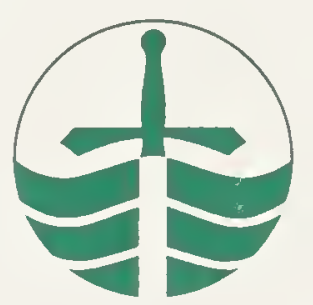

TRENT UNIVERSITY LIBRARY 
Digitized by the Internet Archive in 2019 with funding from Kahle/Austin Foundation 

STANFORD UNIVERSITY PUBLICATIONS U N IVERSITY SERIES

B I O L O G I C A L S C I E N C E S

Volume III

NUMBER 2

\title{
A CLASSIFICATION OF FISHES \\ Including Families and Genera as Far as Known
}

\author{
$B Y$ \\ DAVID STARR JORDAN \\ Chancellor Emeritus of Stanford University
}


QL618.J72

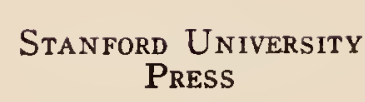

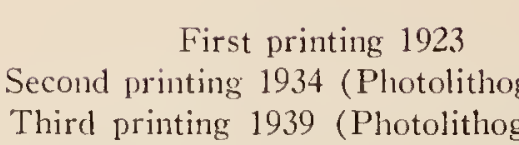




\section{INTRODUCTORY NOTE}

The present paper is a continuation and conclusion to a series of four memoirs issued from 1917 to 1920 under the title of "The Genera of Fishes." In it I have tried to place each genus and subgenus, living and fossil, thus far named or described, whether valid or not, in its proper family and to arrange the families themselves in as natural a sequence as is possible with a linear series which embraces highly divergent lines of evolution. I make no effort to distinguish between valid names, preoccupied names, and synonyms. The multitude of new forms recorded in the last fifty years precludes any such attempt. It will be observed that the number of families I have recognized is, in several groups, greater than accepted by any previous writer. This is due to the fact that analysis must precede synthesis, and it is better to lay a certain stress on aberrant forms than to include them uncritically in expanded groups, the definition of which is impaired or denied by their presence. Investigators who prefer to be more "conservative" can treat these groups as seems best.

In each family I have arranged the genera in the order of time. In most large groups our knowledge is too imperfect to make a detailed natural classification practicable in a compilation like this. In the list, moreover, are many nominal genera, especially among the fossils, in which an arrangement even by families is largely guesswork. As to sequence of orders and families, there is room for wide apparent divergence of opinion. Following recent custom I place the simplest or most ancient forms first, closing with highly specialized types of recent date.

The names of fossil genera are throughout printed in Small Caprtals; those of doubtful eligibility - either because not binomial or else given without type, definition, or explanation-are in Italics. In my personal judgment these stillborn names should not enter the system unless later revived in regular form. Names attached to known species (typonyms) I have, however, admitted without question, and I make, of course, no distinction here between genera and subgenera. In each case I add the date of the genus with the page in "The Genera of Fishes," in which it is more fully treated.

In preparing this paper I have been under special obligation to Allan R. McCulloch of the Australian Museum; to my former student, Carl L. Hubbs of the University of Michigan; and to Theodore D. A. Cockerell of the University of Colorado. Each of these has brought errors or omissions to my attention, and Mr. Hubbs has suggested good reasons for certain important changes in sequence. 
I may again emphasize the distinction between the ancient and modern periods in animal taxonomy. Beginning with 1758, the date of Linnæus' "Systema Naturæ," for sixty years a genus was usually regarded merely as a convenient pigeon-hole into which species are thrown. With Cuvier's "Règne Animal" (1817) came the conception of arrangement "according to organization" or comparative anatomy. With Darwin's "Origin of Species," forty years later (1859), zoologists began, though slowly at first, to realize that a classification must be more than an inventory; that its basis must be genetic, and the problems involved in a natural grouping are vastly more complex than even great morphologists like Cuvier and Agassiz had realized. To paraphrase a saying of Elliott Coues, genera and species are but larger and smaller twigs of a tree which we try to arrange as nearly as possible in accordance ${ }^{a}$ with nature's ramifications. This view applied to taxonomy involves the extensive subdivision of accepted genera, many of which were unnatural in the highest degree, a subdivision in many cases doubtless going too far, but our material must be first analyzed before we can group its parts in natural synthesis. No system of naming can progress beyond the knowledge on which it rests.

I may repeat a warning as old as science itself : that we must not expect a degree of accuracy which the subject in question does not permit.

StaNFORD UNIVERSITY,

David Starr Jordan.

JANUARY 15,1923 


\title{
A CLASSIFICATION OF FISHES
}

\author{
Including Families and Genera as Far as Known
}

\section{Class LEPTOCARDII}

\author{
(Myelozoa)
}

\section{Order AMPHIOXI}

(Cirrostomi)

The Lancelets (or Leptocardii) are without doubt simplest in organization of all fish-like chordates or vertebrates.

Apparently their lowly structure is primitive but the geological history of the group is unknown and likely to remain so, as these creatures possess no hard parts. Whether the lampreys on the one hand or the lower and not fish-like acranite Chordata (Tunicates and Enteropneustans) are derived from Leptocardians can not be known.

\section{Family 1. AMPHIOXIDIDZE}

Amphioxides ${ }^{1}$ Gill, 1895, 467.

${ }^{1}$ This genus of three species, based on the absence of buccal cirri, the unsymmetrical mouth, and some other anatomical details, is now regarded as probably a larval form of Asymmetron rather than as a distinct genus.

\section{Family 2. BRANCHIOSTOMID无 (Amphioxidce)}

\section{(Lancelets)}

Branchiostoma Costa, 1834, 180.

Dolichorhynchus² Willey, 1901, 495.

Amphioxus Yarrell, 1836, 186.

${ }^{2}$ Misprinted Dolichorhamphus by Hubbs, Occ. Papcrs, Univ. Mich., 105, p. 13, 1922.

Family 3. EPIGONICHTHYIDAE

Epigonichthys Peters, 1877, 391.

Paramphioxus ${ }^{4}$ Haeckel, 1893, 461.

Asymmetron ${ }^{3}$ Andrews, 1893.

Heteropleuron ${ }^{4}$ Kirkaldy, 1895, 469.

${ }^{3}$ Studies Johns Hopkins Lab. V, 237; orthotype Asymmetron lucayanum Andrews (overlooked in "Genera of Fishes").

"Paramphioxus and Heteropleuron are synonyms of Epigonichinys. 


\title{
Class MARSIPOBRANCHII
}

\author{
(Myzontes; Cyclostomi; Dermopteri)
}

The Lampreys and Hag-fishes stand next the Lancelets at the foot of the series. They have no hard parts, teeth excepted, and these have never been found fossil.

The name of CONODONTES has been given to a variety of tooth-like structures found in Devonian rocks in Russia and about Lake Erie. But the chemical composition of these differs from that of lamprey-teeth, and it is more likely that they are appendages of Crustaceans or teeth of snails (or worms) rather than of fishes. Most of the generic names given by Pander in 1856, enumerated on page 409 of the "Genera of Fishes," belong to the Palæozoic group of Conodontes; Pander thus classified the Conodonts: (a) Simple structures, slender and sharp, Drepanodus, Acodus, Distacodus, Machairodus, Paltodus, Scolopodus, Oistodus, Acontiodus.

(b) Complex structures, with several cusps. Prioniodus, Belodus, Lonchodus, Ctenognathus, Cordylodus, Gnathodus, Prionognathus, Polygnathus, Centrodus (not of Giebel).

Nothing is known of the geological history of Lampreys, nor can any one say whether Sharks or Ostracophores were in the line of descent from them. The two orders of this class diverge widely from each other, and perhaps, as Lankester has contended, each should be regarded as a distinct class.

\section{Order HYPEROTRETA}

\section{Family 4. MYXINIDEE}

\section{(Hag-fishes)}

Myxine Linnæus, 1758, 406.

Gastrobranchus Bloch, 1797, 53.
Murænoblenna Lacépède, 1803, 68. Anopsus Rafinesque, 1815, 92.

\section{Family 5. EPTATRETID E}

Eptatretus ${ }^{5}$ (Duméril) Cloquet, 1819, 106.

Homea $^{\circ}$ Fleming, 1822.

Heptatrema Duméril, 1856, 268.

Bdellostoma Müller, 1835, 182.

Polistotrema Gill, 1881, 416.

Hexabranchus Schultze, 1835, 184.

This name is often corrected to Heptatretus.

- Philos. Zool. II, 374; type H. banksi Fleming; omitted in "Genera of Fishes"). 


\section{Order HYPEROARTIA}

Family 6. PETROMYZONID王

(Lampreys)

Petromyzon Linnæus, 1758, 11.

Ammocœtus $^{\top}$ Duméril, 1808, 76.

Lampreda Rafinesque, 1815, 93.

Pricus Rafinesque, 1815, 93.

Lampetra Gray, 1851, 249.

Ichthyomyzon Girard, 1859, 291.

Scolecosoma Girard, 1859, 291.

Entosphenus Gill, 1862, 317.

Bathymyzon Gill, 1883, 424.

Caspiomyzon Berg, 1906, 516.
Agnathomyzon Gracianov, 1906, 518.

Haploglossa Gracianov, 1906, 518.

Oceanomyzon Fowler, 1908, 528.

Eudontomyzon Regan, 1911, 540.

Reighardina $^{\text {₹a }}$ Creaser (Charles W.) and Hubbs, 1922.

Tetrapleurodon ${ }^{\text {th }}$ C. \& H., 1922.

Lethenteron $^{\text {2c }}$ C. \& H., 1922.

Okkelbergia $^{\text {ta }}$ C. \& H., 1922.

${ }^{7}$ Usually written Ammocœtes.

7a "A Revision of the Holarctic Lampreys," Occ. Pap. Mus. Zool. Michigan, 120:

2; orthotype Ichthyomyzon fossor Reighard \& Cummins $=$ Petromyzon unicolor Dekay.

${ }^{\text {tb }}$ Loc. cit., 3 ; orthotype Lampetra spadicea Bean.

${ }^{7 c}$ Loc. cit., orthotype Lampetra wilderi Gage $=$ Petromyzon appendix De Kay.

${ }^{r a}$ Loc. cit., 8; orthotype Petromyzon lamotteni Le Sueur $=$ Ammocotes apyptera Abbott.

\section{Family 7. GEOTRIID $\mathbb{E}^{8}$ \\ (Mordaciide)}

Geotria Gray, 1851, 249.

Caragola Gray, 1851, 249.

Velasia Gray, 1851, 249.

Mordacia Gray, 1851, 249.

Thysanochilus Philippi, 1857, 276.
Chilopterus Philippi, 1858, 283.

Exomegas Burmeister, 1868, 348.

Yarra Castelnau, 1872, 363.

Neomordacia Castelnau, 1872, 363.

Macrophthalmia Plate, 1897, 478.

'The name Mordacia may not be tenable, as its synonym Caragola has line priority. 


\section{Class OSTRACOPHORI}

\section{(Aspidoganoidei; Ostracodermi, name preoccupied; Protocephali)}

In regarding the ostracophores as a distinct class I do not pretend to settle any of the vexing questions as to the origin and relations of the group. It may be that the four orders do not belong together, that the Antiarcha are sharks or else Arthrodires, that the group itself is a mailed variant of the Lampreys, that it is ancestral to the sharks, that it is derived from primitive sharks or that it is a variant of primitive crustaceans. In the latter case the ancestry of fishes and through them of other vertebrates, should be sought not in worm-like forms, but in marine creatures with hard shells, related to Limulus and remotely to existing spiders. In general, however, the outer parts are more of ten modified by natural selection than internal structures, hence of less value in determining relations. The outside of an animal tells where it has been, the inside what it really is.

In Hæckel's words, there are three ancestral documents in the study of evolution, morphology, embryology, and palæontology. From the first two no decisive answer is given to the question of the origin of the ostracophores. Paleontology shows that several of the great divisions of fishes had appeared in the Devoniar. The oldest known fragment of a fish (Astraspis) is from the Ordovician in the Trenton horizon at Cañon City, Colorado. With this, however, are other fragments doubtfully referred to a chimara (Dictyorhabdus) and a crossopterygian (Eriptychius). If these are correctly identified the differentiation of fishes had proceeded far when the Palæozoic era began.

Most naturalists have traced the hypothetic ancestry of vertebrates through lancelets or enteropneustans back to soft-bodied, worm-like forms which have left no traces in the rocks. Dr. Patten, however, regards Pteraspis as descended from primitive arachnids with bony carapace. From Pteraspis he derives the other ostracophores, and from these the sharks and other vertebrates appearing progressively later in time. This ingenious theory is developed in the American Naturalist, 1904. Patten regards the lateral fold in some sharks as the result of a fusion of the fringing appendages on the sides of the body. This is evident in his restoration of Cepinalaspis. 


\title{
Order HETEROSTRACI
}

\author{
(Aspidorlini)
}

\section{Family 8. THELODONTID王 (Cololcpide)} family.

Thelodus is identical with Cololepis and should give the name to this

Thelodus Agassiz, 1839, 194.

Celolepis Pander, 1856, 273.

Nostoleris Pander, 1856, 274.

PACH ylepis Pander, 1856, 274.

Thelolepis Pander, 1856, 274.

Gomphodus Pander, 1856, 274.

Schedrosteus Pander, 1856, 273.
Dasylepis, Pander, 1856, 273.

Dictyolepis Pander, 1856, 273.

Oniscolemis Pander, 1856, 273.

Melitromalepis Pander, 1856, 273.

Prionacanthus Pander; 1856, 273.

Spirodus Kade, 1858, 281.

LANARKIA Traquair, 1899, 488.

\section{Family 9. PSAMMOSTEID丑}

Psammolepis Agassiz, (1844) 1845, 217.

Placosteus Agassiz, (1844) 1845, 217.

Psammosteus Agassiz, 1845, 225.

Lophosteus Pander, 1856, 274.

Acanthaspis Newberry, 1875, 379.
Acantholepis Newberry, 1875, 379.

Ganosteus Rohon, 1902, 500.

Pycnosteus Preobrazhenskii, 1910, 537.

Dyptychosteus Preobrazhenskii, 1910, 537.

\section{Family 10. ASTRASPID在}

Astraspis $^{10}$ Walcott, 1892, 459.

${ }^{10}$ See Eastman, Proc. U. S. Nat. Mus., 52: 237, 1917.

\section{Family 11. DREPANASPID FE}

Drepanaspis Schlüter, 1887, 439.

\section{Family 12. CERASPIDE}

CERAsPis Schlüter, 1887, 439.

\section{Family 13. PTERASPIDE}

SPhagodus ${ }^{11}$ Agassiz, 1839, 194.

CHIAstolepis ${ }^{12}$ Eichwald, 1844, 220.

PterAspis Kner, 1847, 232.

ARCh жотеUthis Roemer, 1855, 264.

Paleoteuthis Roemer, 1855, 266.

Tolypelepis Pander, 1856, 273.

SCAPHASPIS Lankester, 1865, 338.
Cyathaspis ${ }^{13}$ Lankester, 1865.

Kallostrakon Lankester, 1870, 358.

HoldASPIS $^{14}$ Lankester, 1873, 370.

Paleaspis Claypole, 1884, 427, 430.

Diplaspis Matthew, 1887, 438.

Tolypaspis Schmidt, 1893, 462.

Pholidosteus Jaekel, 1907, 524.

${ }^{11}$ A very doubtful genus, the type said to be part of the mouth of an Eurypterid Crustacean, as is also a tooth called Aulaconus.

${ }^{12}$ A very doubtful fragment of armor.

${ }^{13}$ Rep. Brit. Assoc. for 1864, p. 58. Omitted in "Genera of Fishes": type Pteraspis banksi Huxley \& Salter.

14 Preoccupied by Holaspis Gray, 1863, a reptile. 


\section{Order OSTEOSTRACI}

\section{Family 14. CEPHALASPID $\mathbb{E}$}

Cephalaspis Agassiz, 1835, 181.

ZEN Aspis Lankester, 1850, 246.

Steganodictyum M'Coy, 1851, 249.

\section{Family 15. ATELEASPIDAE}

Ateleaspis Traquair, 1899, 488.

\section{Family 16. THYESTIDÆ (Menaspida)}

Sclerodus Agassiz, 1839, 194.

Plectrodus Agassiz, 1839, 194.

Menaspis Ewald 1848, 233.

Thyestes Eichwald, 1854, 256.

Auchenaspis Egerton, 1857, 275.

DIDYMASPIS Lankester, 1867, 346.

EukERASPIs Lankester, 1870, 358.

Cephalopterus Powrie, 1870, 359.

\section{Family 17. ODONTODONTIDÆ (Tremataspida)}

The name Odontotodus has priority over Tremataspis.

Stigmolepis Pander, 1856, 273.

Odontotodus Pander, 1856, 274.

Coscinodus ${ }^{14 n}$ Pander, 1856.

Monopleurodus ${ }^{14 a}$ Pander, 1856, 273.
Strosispherus ${ }^{14}$ Pander, 1856, 274.

Tremataspis ${ }^{15}$ Schmidt, 1866, 343.

Ancistrodus $^{14 a}$ Rohon, 1893, 462.

${ }^{14 a}$ The relationship of these forms is apparently quite unknown.

${ }^{15}$ The genus Mycterops Cope (1886) should be erased. According to Woodward it is based on a shield of an Eurypterid Crustacean.

\section{Family 18. EUPHANEROPID压}

Euphanerops Woodward, 1900, 491.

\section{Order ANTIARCHA}

(Placodermi part; Placoganoidei; Azygostei part)

It is not yet certain that this order should be retained among the ostracophores. Hay and others place it with the arthrodires in a subclass Azygostei or a superorder Placodermi.

\section{Family 19. ASTROLEPID瓜 (Bothrolepida; Pterichthyida)}

Pleiopterus Agassiz, 1835, 181.

Astrolepis ${ }^{16}$ Eichwald, 1840, 206.

Bothriolepis Eichwald, 1840, 206.

Pterichthys Hugh Miller, 1841, 209.

Placothorax Agassiz, 1844, 218.

Pamphractus Agassiz, 1844, 218.

Homothorax Agassiz, 1845, 225.
Odontacanthus Agassiz, 1845, 225.

Narcodes Agassiz, 1845, 225.

Actinolepis Agassiz, 1845, 225.

Pterichth yodes Bleeker, 1859, 289.

Microbrachium Traquair, 1888, 443.

Glyptosteus Agassiz, (1844) 1891, 217.

Millerichthys S. A. Miller, 1892, 458.

\footnotetext{
${ }^{16}$ Usually written Asterolepis.
} 


\title{
Order ANASPIDA
}

\author{
Family 20. LASANIID无
}

Lasanius Traquair, 1899, 488.

Family 21. BIRKENIID压

Birkenia Traquair, 1899, 488.

Ctenopleuron Matthew, 1907, 525.

\section{Order CYCLIAE}

\section{Family 22. PALAOSPONDYLID $\mathbb{E}$}

I here give the rank of a separate order to the singular little fish-like organism of the Scottish Devonian age known as Palaospondylus gunni. In doing so, I do not assume to decide what its relationships may be, whether a minute but specialized primitive lamprey, an offshoot from the arthrodires or the ostracophores or whatever else may be possible. Perhaps, as suggested by Huxley, it may be a "baby Coccosteus."

Paleospondylus Traquair, 1890, 453. 


\section{Class ARTHRODIRA}

Order STEGOPHTHALAMI

Family 23. MACROPETALICHTHYIDA

Macropetalichtizs Norwood and Owen, 1846, 229.

Family 24. ASTEROSTEIDE

Asterosteus Newberry, 1875, 379.

\section{Order TEMNOTHORACI}

\section{Family 25. CHELONICHTHYID Z2⿰}

ICHTH yoSAUroides Kutorga, "1837, 189. Honosteus ${ }^{17}$ Asmuss, 1856, 267.
Heterosteus Asmuss, 1856, 267.

Chelonichthys ${ }^{18 z}$ Agassiz, (1842) 1891, 217.

\footnotetext{
${ }^{16 a}$ Agassiz quotes this name from "Murchison's Report, 1842.

${ }^{17}$ Name misprinted Homostius.
}

\section{Order ARTHROTHORACI}

\section{Family 26. COCCOSTEIDE}

Coccosteus Agassiz, 1844, 218.

Cinelyophorus Agassiz, 1845, 225.

Siphonodus, Fischer von Waldheim, $1852,250$.

Physichthys Meyer, 1855, 266.

AgAssichthys Newberry, 1857, 276.

Aspidophorus Newberry, 1870, 359.

A spidichthys Newberry, $1873,371$.

Lioginathus Newberry, 1873, 371.

BRACriydruU $^{18}$ Koenen, 1880, 404.

Anomalichthys Koenen, 1883, 424.
Lophostracon Lankester, 1884, 429.

Titanichthys ${ }^{18}$ Dames, 1887, 437.

Gigantichthys Dames, 1888, 440.

Glyptaspis Newberry, 1889, 447.

Trachosteus Newberry, 1889, 447.

Phlyctanius Traquair, $1890,452$.

Phimctanaspis Traquair, 1890, 453.

Asteroplax Woodward, 1891, 456.

Ponerichthys Miller, 1892, 458.

Lispognathus Miller, 1892, 458.

${ }^{x 8}$ The name Leptodus was based on a Brachiopod shell and should be erased.

"According to Regan, as quoted by Cockerell, Gigantichthys (Titanichthys Dames, not Newberry) is based on a Reptilian tooth.

\section{Family 27. DINICHTHXIDAE}

Dinichtemys ${ }^{20}$ Newberry, $1868,371$.

Gorgonichthys Claypole, $1892,457$.

Brontichthys Claypole, 1894, 464.

Platyaspis Koenen, 1895, 469.

Stenognathus Newberry, 1898, 479, 483.

Phlyctanium Zittel, 1900, 491.

Pachyosteus Jaekel, 1903, 503.

BRACHYGNATHUS Hussakof, 1909, 533.
Hussamofia Cossman, 1910, 535.

Copanognathus Hussakof and Bryant, 1919, 566.

Mach zirognathus Hussakof and Bryant, 1919, 566.

Perissognathus Hussakof and Bryant, 1919, 566.

\footnotetext{
${ }^{20}$ Proc. Amer. Asso. Adv. Sci. for 1867, 17 : 148 (1868).
} 


\section{Family 28. TITANICHTHYID $\mathbb{E}$}

Titanichthys Newberry, 1885, $433 . \quad$ Protitanichthys Eastman, 1907, 521.

Holopetalichthys Koenen, 1895, 469.

\section{Family 29. MYLOSTOMID㤰}

Typodus $^{21}$ Meyer, 1847, 232.

Dinomylostoma Eastman, 1906, 517.

Mylostoma Newberry, 1883, 425.

Dinognathus Hussakof, 1909, 533.

${ }^{21}$ Misprinted Tylodus in "Genera of Fishes," p. 232.

\section{Family 30. SELENOSTEID}

Diplognathus Newberry, 1878, $397 . \quad$ Stenosteus Dean, 1901, 492.

Selenosteus Dean, 1901, 492.

\section{Family 31. PHYLLOLEPID开}

Phyllolepis Agassiz, 1844, 219.

Holonema Newberry, 1889, 447. 


\title{
Class ELASMOBRANCHII
}

\author{
(Selachii; Plagiostomata; Chondropterygii; Antacea)
}

\section{Subclass SELACHII}

\section{(Sharks)}

Whatever may have been the origin of sharks, whether from lampreylike or other soft bodied forms, or through degeneration from more specialized types, I have little doubt that the most primitive form is the order Pleuropterygii. It seems to me, as to Dr. Dean, that the pectoral fin of Cladoselache, apparently derived or derivable from a lateral fold, comes nearest the primitive condition. It is conceivable that the robust pectoral spine of Acanthoëssus is homologous with the fin of Cladoselache, the anterior fin-rays being gathered together to be united in a thick spine.

If, however, we regard the pectoral limb as developed from gillstructures, the conception of origin would be changed. The jointed limb of Orthacanthus would be the nearest approach to the primitive pectoral limb, and from the ancestors or allies of the Ichthyotomi all other sharks and through them all the higher fishes as well as the higher vertebrates would be descended. Whether the pectoral limb or archipterygium of the Crossopterygii or the Dipneusti is homologous with that of the Ichthyotomi, or separately derived is not yet known.

The weight of authority, at present, favors the theory that the paired fins are derived from a fold of skin, and that of the known forms Cladoselache of the Devonian is the most primitive. We therefore adopt a sequence in accord with this theory, recognizing at the same time that weight of authority is unimportant when decisive evidence is wanting.

Leaving aside certain ichthyodorulites or detached fin-spines with other fragments of uncertain character the earliest known sharks (Acanthoësside) appear in the lower Devonian, while Orthacanthus and certain Heterodontids occur in the Carboniferous. In the Triassic all the early forms of sharks, Orodontide excepted, disappear, and all or nearly all the living forms are descended from this Heterodontid type. To this statement the Notidani with Chlamydoselachus may form an exception, for their peculiar traits seem to be primitive, though no specimens are found before the Jurassic.

The chimæras represent a very early divergence from the sharks, being apparently nearly as old geologically as the sharks. If Dictyorhabdus from the Ordovician is really a chimæra, which Dr. Dean doubts, these fishes are older than any known sharks. 


\title{
Order PLEUROPTERYGII
}

\author{
(Cladoselachea)
}

Family 32. CLADOSELACHID正

Cladoselache ${ }^{22}$ Dean, 1894.

"Contributions to the Morphology of Cladoselache," Journ. Morphol., 9:87; type Cladodus fyleri Newberry. (Omitted in "Genera of Fishes.")

\section{Order ACANTHODEI}

This order does not differ widely from the Pleuropterygii. If we assume that its fin spines are simply massed rays, as Dr. Dean asserts, the essential structure of the two groups is identical.

\section{Family 33. ACANTHÖ̈SSID无 (Acanthodide)}

The name Acanthoëssus has priority over Acanthodes. Those genera marked Ich are known only from Ichthyodorulites, fin spines of more or less uncertain relations.

ACANтrö̈ssus Agassiz, 1832, 141.

Acanthodes Agassiz, 1833, 145, 177.

Cheiracanthus Agassiz, 1835, 181.

Haplacanthus Agassiz, 1845, 225.

Homacanthus Agassiz, 1845, 225, Ich.

Byssacanthus Agassiz, 1845, 225, Ich.

Holacanthodes Beyrich, 1848, 233.

Pelonectes Gistel, 1848, 234.

Macheracanthus Newberty, 1857, 276 Ich.
MACh ERIUS Rouault, 1858, 284, Ich.

Acanthodopsis Hancock and Atthey, 1868, 352.

Rhadinacanthus Traquair, 1888, 443.

Mesacanthus Traquair, 1888, 443.

HeteraCANThus ${ }^{23}$ Newberry, 1889, 447

Ich.

Traquairia Fritsch, 1893, 460.

Protacanthus Fritsch, 1893, 460. 1836.

${ }^{2}$ Ganiodus Newberry should be erased. It is a misprint for Goniodus Agassiz, Family 34. ISCHNACANTHIDE (Ictinocephalida) Ischnacanthus ${ }^{24}$ Powrie, $1864 . \quad$ Ictinocephalus Page, (1859) 1864, 334.

${ }^{24}$ Quart. Journ. Geol., 419, 1864; type Diplacanthus gracilis Egerton. (Omitted in "Genera of Fishes.")

Family 35. DIPLACANTHIDÆ

Diplacanthus Agassiz, 1842, 210, 225.

Parexus Agassiz, 1845; 225.

Climatius Agassiz, 1845, 225.
Brachyacanthus Egerton, 1860, 295.

Euthacanthus Powrie, 1864, 334.

Marsdenius Wellburn, 1902, 501.

\section{Order ICHTHYOTOMI}

\section{Family 36. XENACANTHIDE (Pleuracanthida; Orthacanthida)}

The name Pleuracanthus is preoccupied (Coleoptera Gray, 1832), as is also Diplodus Agassiz. The name next oldest, Orthacanthus, seems to 
belong to a different genus, while Xenacanthus appears to be an exact synonym of Plcuracanthus.

Pleuracanthus Agassiz, 1837, 186.

Diplodus Agassiz, 1843, 213.

Orthacanthus Agassiz, 1843, 213.

Acanthopleurus Agassiz, 1844, 217.

Xenacanthus Beyrich, 1848, 233.

Triodus Jordan, 1849, 243.

Compsacanthus Newberry, 1856, 273.

Dittonus Owen, 1867, 346.

Ochlodus Owen, $1867,347$.

Aganodus Owen, 1867, 347
Pternodus Owen, 1867, 347.

Thrinacodus St. John and Worthen, $1875,380$.

Phricacanthus Davis, 1879, 399.

Lophacanthus Stock, 1880, 406.

Anodontacanthus Davis, 1881, 415.

Didymodus Cope, $1883,422$.

Diacranodus ${ }^{25}$ Garman, 1885, 432.

Doliodus Traquair, 1892, 459.

${ }^{25}$ Misprinted Dicranodus in "Genera of Fishes."

Family 37. CLADODONTIDÆ

Clanodus Agassiz, 1843, 213.

Crillodus Giebel, 1847, 231.

Phcerodus St. John and Worthen, 1875 , 380.

LAMBdodus St. John and Worthen, 1875 , 380.

Bathycheilodus St. John and Worthen, 1875,380 .
Hybocladodus $^{20}$ St. John and Worthen, $1875,380$.

Pternodus Garman, 1885, 432.

Dicentrodus Traquair, 1888, 443.

Styptobasis Cope, 1891, 454.

Protodus Woodward, 1892, 459.

Monocladodus Claypole, 1893, 460.

Symmorium Cope, 1893, 460.

${ }^{26}$ The insertion of Hybocladodus Hay, 1899, in the "Genera of Fishes" is an error.

Family 38. CHONDRENCHELYID压

Chondrenchelys Traquair, 1888, 443.

Family 39. GYRACANTHID $Æ$

Gyracanthus Agassiz, 1837, 186.

Mitrodus Owen, 1867, 347.

\section{Order POLYSPONDYLI}

We may here insert the hypothetical order Polyspondyli, regarded by Hasse as a primitive type known from fin-spines or Ichthydorulites only, and these all of uncertain relationship, some of them perhaps Heterodontid.

Onchus occurs in the upper Silurian.

\section{Family 40. ONCHID叛}

Onchus Agassiz, 1837, 186.

Naulas Agassiz, 1845, 225.
Leptocheles M'Coy, 1853, 408.

Arch Æacanthus Kade, 1858, 281.

\section{Order CESTRACIONTES}

\section{Suborder JANASSIDES}

I here arrange provisionally certain families of sharks, some or all with flattened bodies like the rays, but occurring in Palæozoic time they are probably not genetically connected with the flattened skates and rays of 
recent times. Except the genus Janassa, the species are mostly known from teeth only, these mostly large and fitted for grinding.

\section{Family 41. JANASSID压 (Petalodontida)}

JANASSA Münster, 1832, 176.

Ctenoptychius Agassiz, 1838, 190.

Petalodus $^{27}$ Owen, 1840, 207.

Dictea Münster, 1840, 207.

Byzeños Münster, 1843, 216.

Carcharopsis (Agassiz) Davis, (1843) $1883,214,432$.

Cimmaxodus M'Coy, 1848, 239.

Glossonus M'Coy, 1848, 239.

Polyrhizodus M'Coy, 1848, 239.

Platacanthus ${ }^{28}$ M'Coy, 1848, 408.

Dicrenodus Romanovskii, 1853, 254.

Pristicladodus M'Coy, 1855, 266.

Sicarius Leidy, 1856, 272.

Xystracanthus Leidy, 1859, 292.

Ctenopetalus (Agassiz) Morris \& Roberts (1862) 1881, 320.

Harpacodus (Agassiz) M. \& R., (1862) 1881, 320, 415.

Petalorhynchus (Agassiz) M. \& R., (1862) $1881,320$.

Dactylodus Newberry and Worthen, $1866,342$.

ANtLiodus N. \& W., 1866, 349.

Ageleodus Owen, $1867,347$.

Cymatodus N. \& W., 1870, 359.

Peltodus N. \& W., 1870, 359.
Petalodopsis Barkas, 1874, 372.

Lisgodus St. John \& Worthen, 1875, 380.

Tanaodus St. J. \& W., 1875, 380.

Harpacodus (Agassiz) St. J. \& W., 1875, 380.

Cholodus St. J. \& W., 1875, 380.

Calopodus St. J. \& W., 1875, 380

Fissodus St. J. \& W., 1875, 380.

Batacanthus St. J. \& W., 1875, 381, 425.

Antacanthus ${ }^{29}$ Devalque, 1877.

Strigilina Cope, 1877, 388.

Serratodus Koninck, 1878, 396.

Stich æacanthus Koninck, 1878, 396.

Cymatodus Trautschold, 1879, 401.

Glyphanodus Davis, 1883, 422.

Phoderacanthus Davis, 1883, 423.

Petalodopsis Davis, 1883, 423.

Pnigeacantuus St. John \& Worthen, 1883, 425.

Thoracodus Cope, 1883, 422.

Gomphacanthus Davis, 1884, 427.

Aganacanthus Traquair, 1884, 430.

Callopristodus Traquair, 1888, 443.

Mesolophodus Woodward, 1889, 449.

Stethacanthus Newberry, $1889,447$.

Gamphacanthus Miller, 1892, 458.

Paracymatodus Bogoliubov, 1914, 552.

\footnotetext{
${ }^{27}$ Perhaps a synonym of Ctenoptychilus.

${ }^{28}$ Later amended to Platyacanthus.

${ }^{20}$ Ann. Soc. Geol. Belge, 5:60; type A. insignis Devalque (omitted in "Genera of Fishes").
}

\section{Family 42. PSAMMODONTID $\#$}

Psammodus ${ }^{30}$ Agassiz, 1838.

A podo metes Gistel, 1848, 237.

PaL EOBATES Meyer, 1849, 243.

Characodus (Agassiz) Morris \& Roberts, 1862, 320, 423.

Mylax (Agassiz) M. \& R., 1862, 320.

Pleurogomphus (Agassiz) M. \& R., (1862) $1883,320$.

Mesogomphus (Agassiz) M. \& R., (1862) 1883, 320.

LoBodus (Agassiz) M. \& R., (1862) 1883,320 .
Corodus (Agassiz) M. \& R., (1862), $1883,320,422$.

Solenodus, Trautschold, 1874, 377.

Arcineobatis Newberry, 1878, 397.

Dimyleus (Agassiz) Davis, 1883, 423.

Mylacodus Davis, 1883, 423.

Pinacodus (Agassiz), Davis, 1883, 423.

Homalonus Davis, 1883, 423.

Rhymodus (Agassiz) Davis, 1883, 423.

Astrabodus Davis, 1884, 427.

Mazodus Newberry, 1889, 447.

Lagaronus Jaekel, 1898, 480.

${ }^{30}$ Poissons Fiossiles, 3: 110; type P. rugosus Agassiz (omitted in "Genera of Fishes"). 


\section{Family 43. PERIPRISTID瓜 (Pristodontide)}

The name Peripristis is earlier than Pristodus and belongs to the same genus.

Peripristis ${ }^{31}$ St. John, 1870, 359.

Hoplodus ${ }^{32}$ Etheridge, 1881.
Dionontopsonus Davis, 1881, 415.

Pristodus (Agassiz) Davis, 1883, 424.

${ }^{31}$ Identical with Pristodus, not with Ctenoptychius as stated in "Genera of Fishes."

${ }^{22}$ R. Etheridge, Jr. Geol. Mag. (2) 2: 244, 1825; type Petalorhynchus benniei Etheridge (omitted in "Genera of Fishes").

\section{Family 44. COCHLIODONTIDE}

This family, known only from the teeth, fitted for crushing shells, may be left provisionally with the Janassides, although it has much in common with the Cestraciontes or Heterodontidce. The species belong mostly to the Carboniferous age.

Cochlionus Agassiz, 1838, 191.

Chomatodus Agassiz, 1838, 190.

Helodus Agassiz, 1838, 190.

Pecilodus M'Coy, 1855, 266.

Dichelodus Giebel, 1857, 409.

Psephodus (Agassiz) Morris \& Roberts, (1862) $1883,320$.

Streblonus (Agassiz) M. \& R., (1862) $1883,320$.

Tomodus (Agassiz) M. \& R., (1862) $1883,422$.

Xystrodus (Agassiz) M. \& R., (1862) $1883,320$.

Deltoptychius (Agassiz) M. \& R., (1862) $1883,320$.

Deltodus (Agassiz) M. \& R., (1862) 1883, 319.

Lophodus Romanowsky, 1864, 334.

Trigonodus Niewberry \& Worthen, 1866, 342.

Aspidonus N. \& W., 1866, 342.

Sandalodus N. \& W., 1866, 342.

Pleurodus Hancock \& Atthey, 1872, 366, 213.
Venustodus St. John \& Worthen, 1875, 380.

Periplectronus St. J. \& W., 1875, 380.

Platyodus Newberry, 1875, 379.

Helodopsis Waagen, 1879, 401.

Cranodus Trautschold, 1879, 401.

Oxyтомоdus Trautschold, 1880, 406.

VAticinodus ${ }^{33}$ St. J. \& W., 1883, 405, 425.

Stenopterodus ${ }^{34}$ St. J. \& W., 1883, 405, 425.

Chitonodus ${ }^{36}$ St. J. \& W., 1883, 405, 425.

Deltodopsis ${ }^{36}$ St. J. \& W., 1883, 405, 425.

Orthopleurodus ${ }^{37}$ St. J. \& W., 1880, 405, 425.

TæNiodus St. J. \& W., 1883, 425.

Rhampiodus Davis, 1883, 422.

Diplacodus Davis, 1884, 427.

Cyrtonodus Davis, 1884, 427.

Pleuroplax Woodward, 1889, 449.

Xenodus Miller, 1892, 458.

ICANODUs Miller, 1892, 458.

Platyxystrodus Hay, 1899, 486.

\footnotetext{
${ }^{33}$ Logotype $V$. vetustus St. J. \& W. The date of 1880 given in "Genera of Fishes" is an error.

${ }^{84}$ Logotype S. planus St. J. \& W., as fixed by Hay.

${ }^{35}$ Logotype C. springeri St. J. \& WW.

${ }^{86}$ Orthotype $D$. augustus St. J. \& W.

${ }^{37}$ Orthotype O. carbonarius St. J. \& W.
} 


\section{Family 45. TAMIOBATID $Æ$}

This family is known from a single species, from the Devonian of Kentucky, one of the oldest sharks known. It has the depressed form of a ray, probably a matter of analogy only.

Tam iobatis Eastman, 1897, 476.

\section{Suborder PROSARTHRI}

\section{Family 46. ORODONTIDE}

The Orodontida, allies or ancestors of the Hetcrodontida, are the only kinds of sharks living in the Triassic, all the earlier types being then extinct.

Orodus Agassiz, 1838, 190.

CAM Podus Koninck, 1844, 220.

Asteroptycinus Agassiz, 1848, 2.39.

Adiapneustes Gistel, 1848, 234.

Petrodus M'Coy, 1848, 239.

Octinaspis Trautschold, 1874, 377.

Mesodmodus St. John \& Worthen, 1875, 380.
Acondylacanthus St. J. \& W., 1875, 380.

Stemmatodus St. J. \& W., $1875,380$.

Leiodus St. J. \& W., 1875, 380.

Desmiodus St. J. \& W., 1875, 380.

Stem matias Hay, 1899, 486.

Eoorodus Branson, 1914, 552.

\section{Family 47. HETERODONTID $\mathbb{E}$ (Cestraciontida, Hybodontida)}

\section{(Bull-head Sharks)}

To this family, characterized among other features by strong dorsal spines, many of the Ichthyodorulites are supposed to belong. Those genera of very uncertain relations are marked $I c h$.

Cestracion Klein, 1775, 40.

Heterodontus Blainville, 1816, 95.

Cestracion Cuvier, 1817, 97.

Centracion Gray; 1831, 138.

TrISTyChiUs $^{38}$ Agassiz, 1837, 186.

Ptychacanthus Agassiz, 1837, 186.

Sphenacanthus Agassiz, 1837, 186.

Asteracanthus Agassiz, 1837, 186.

Leiacantuus Agassiz, 1837, 186.

Нyвоdus $^{39}$ Agassiz, 1837, 186.

Acrodus Agassiz, 1837, 189, 191.

Ctenacanthus Agassiz, 1837, 186, Ich.

Nemacanthus Agassiz, 1837, 186, Ich.

Strophodus Agassiz, 1838, 191.

Oroptychus $^{\text {*0 Agassiz, } 1842 .}$

Meristodon Agassiz, 1843, 214.
Sphenonchus Agassiz, 1843, 213.

WODNIKA ${ }^{41}$ Münster, 1843, 216.

Thectodus Meyer \& Plieninger, 1844, 221.

Centrodus Giebel, 1847, 231.

Centrodus M'Coy, 1848, 243, 408.

Physonemus M'Coy, 1848, 239, Ich.

Styracodus Germar, 1849, 242.

SelachideA Quenstedt, 1852, 251.

Pycnacanthus Fischer von Waldheim, 1852, 250, Ich.

Desmacanthus Quenstedt, 1858, 283, Ich.

Psilacanthus Quenstedt, 1858, 283, Ich.

Xystrodus Plieninger, 1860, 298.

LopHodus Symonds, 1861, 309.

Gyropleurodus Gill, 1862, 317.

Tropidodus Gill, 1862, 317.

${ }^{38}$ Tristychius Portlock should be omitted as an error for Tristychius Agassiz.

${ }^{30}$ Stensiö (1921) regards Hybodus reticulatus Agassiz as the type of this genus.

${ }^{4}$ A manuscript name, apparently never defined, quoted in the Nomenclator Zoologicus from Misc. Coll. as one of the "Cestraciontes."

"The so-called Wodnika ocoye Jordan, "Fossil Fishes of Southern California," 1919,69 , from California Miocene proves to be not a tooth but a concretion. 
Nemacanthus Mackie, 1863, 327.

Curtodus Sauvage, 1867, 347.

LePracanthus Owen, 1869, 355, Ich.

Lophodus Newberry, 1870, 359.

Paleospinax Egerton, 1872, 365.

Drepanephorus Egerton, 1872, 365.

Agassizonus St. John \& Worthen, 1875, 380.

A macanthus St. J. \& W., 1875, 381, Ich.

Bythiacanthus St. J. \& W., 1875, 381.

Glymmatacanthus ${ }^{42}$ St. J. \& W., 1875, 381,425 , Ich.

Geisacanthus St. J. \& W., 1875, 381, Ich.

Hoplonchus Davis, 1876, 385, Ich.

Hybodopsis Barkas, 1878, 392.

Tomodus $^{\star 3}$ Trautschold, 1879.

Chiastodus Trautschold, 1879, 401.

Arpagodus Trautschold, 1879, 401.

Thaumatacanthus Waagen, 1879,401 , Ich.

OxутомоDUs ${ }^{44}$ Trautschold, 1880.
Bdellodus Quenstedt, 1882, 421.

Meristodon Sauvage, 1883, 425.

Diclitodus Davis, 1883, 422.

Eunemacanthus St. J. \& W., 1883, 425, Ich.

Acondylacanthus St. J. \& W., 1883, 425, Ich.

Lispacanthus Davis, 1883,422 , Ich.

Citalazacanthus Davis, 1883,422 , Ich.

Echinodus Davis, 1884, 427.

Synechodus Woodward, 1888, 444.

Acrodonchus Fraas, 1889, 446.

Polyacrodus Jaekel, 1889, 447.

Hybodonchus Fraas, 1889, 446.

FAyolia Renault \& Zeiller, 1890, 452.

Rhabdiodus Rohon, 1893, 462.

Euphyacanthus Traquair, 1894, 465.

Ortri ybodus Jaekel, 1898, 480.

Parhybodus Jaekel, 1898, 480.

Pseudacrodus Ameghino, 1906, 516.

${ }^{42}$ Misprinted in "Genera of Fishes."

"Nouv. Mem. Soc. Imp. Nat. Moscow, 14: 55; type T. argutus Trautschold (omitted in "Genera of Fishes").

"Bull. Soc. Imp. Nat. Moscoze 2: 140; substitute for Tomodis, preoccupied; type Tomodus argutus Trautschold. (Omitted in "Genera of Fishes.")

\section{Family 48. EDESTIDE .}

A peculiar type of sharks, known only by singular bony structures now supposed fused symphyseal whorls of teeth of cestraciont sharks.

Edestus Leidy, 1856, 272.

Cynopodius Traquair, 1881, 417.

Euctenius Traquair, 1881, 417.

SpIraxis Newberry, 1885 , 434, Ich.

Prospiraxis Williams, $1887,439$.

Protopirata Trautschold, 1888, 443.
SPHenophorus Newberry, 1889, 447.

Estophorus Miller, 1892, 458.

HeLICOPRION Karpinskii, 1899, 487.

CAMPYLOPRION Eastman, 1902, 498.

Lissoprion Hay, 1907, 524.

Toxoprion Hay, 1910, 536.

\section{Series ICHTHYODORULITES}

(Shark-spines)

We place here a number of fin spines belonging perhaps to Heterodonti$d a$ or to Chimarida, their relationship not yet determined.

Pristacanthus Agassiz, 1837, 186, Ich. Cricacanthus ${ }^{45}$ Agassiz, 1843, 213, Ich. Cosmacanthus Agassiz, 1845, 225, Ich. Dimeracanthus Keyserling, 1846, 229, Ich.

Gyropristis" (Agassiz) King, (1843) 1850,213 , Ich.

Rytidolepis Pander, 1856, 273, Ich.
Stenacanthus Leidy, 1856, 273, Ich. Marracanthus St. John \& Worthen, 1875,381 , Ich.

Compsacanthus Davis, 1883,422 , Ich. Brachiacantius Fritsch, 1889, 446, Ich. Tubulacanthus Fritsch, 1889, 446, Ich. Platyacanthius Fritsch, 1889, 446, Ich. Callognathus Newberry, 1889, 447, Ich. 
Phlyctenacanthus Eastman, 1898, 479, Ich.

Eucentrurus Traquair, 1901, 495, Ich.

EuCtenodopsis Wellburn, 1901, 495.
Eczematolepis Miller, 1892, 458, Ich. Atopacantrus Hussakof \& Bryant, 1919, 566, Ich.

${ }^{45}$ Name only ; the species, $C$. jonesi Agassiz, misprinted in "Genera of Fishes," 213.

* Name only; used in 1850 by W. King: "Permian Fossils," Pal. Soc. 1850, 222.

\section{Order SELACHOPHIDICHTHYOIDEI}

\section{(Pternodonta)}

This order and the next seem to find place somewhere between the Cladoselachide and the Hetcrodontida, although so far as geological evidence both groups long preceded them in time.

\section{Family 49. CHLAMYDOSELACHID $A$ E}

(Frilled Sharks)

Chlamydoselachus Garman, 1884, 428.

\section{Order NOTIDANI \\ (Diplospondyli; Protoselachii) \\ Suborder OPISTHARTHRI \\ Family 50. HEXANCHID $\approx$ \\ (Cow Sharks)}

Hexanchus Rafinesque, 1810, 78.

Monopterhinus Blainville, 1816, 95.

Notidanus Cuvier, 1817, 97.

Holodus $^{48}$ Agassiz, 1842.
Heptranchias ${ }^{47}$ Rafinesque, $1810,78$.

Notorhynchus Ayres, 1855, 262.

Notidanion $^{48 a}$ Jordan \& Hannibal, 1923.

${ }^{47}$ Sometimes perverted to Hepianchus, the original name a condensation of Heptabranchus, seven-gilled.

${ }^{48}$ Apparently a manuscript name never defined, quoted from "Misc. Coll." in the Nomenclator Zoologicus, Appendix, p. 3, as equivalent to Notidanus.

${ }^{48}$ Notidanion Jordan \& Hannibal, new genus; orthotype Notidanus primigenius Agassiz, Poisson Fossiles, plate 27, fig. 9: "Lateral teeth with the first cusp very large, the others few and large, 4 to 6 in number; anterior serrations very coarse."

\section{Order EUSELACHII}

\section{Suborder GALEI}

(Scyllioidei)

\section{Family 51. HEMISCYLIIID E}

The sharks of this family are ovoviviparous, those of the other scyllioid genera have large oblong quadrate leathery egg-cases, with filaments at the corners. Some of the genera enumerated among the Scylliorhinida may prove to belong here.

Hemiscyllium A. Smith, 1837, 190.

Cirrhoscyllium H. M. Smith, 1913, 551.

Chiloscyllium Müller \& Henle, 1838, 192. 


\section{Family 52. SCYLLIORHINID $\mathbb{E}$}

(Cat-Sharks)

Catulus Valmont, 1768, 27.

Scylliorhinus Blainville, 1816, 95.

Scyllium Cuvier, 1817, 97.

Catulus Smith, 1837, 190.

Poroderma Smith, 1837, 190.

Pristiurus Müller \& Henle, 1838, 573.

Pristidurus Bonaparte, 1839, 194.

Pristiurus Bonaparte, 1841, 208.

Thyelinina Agassiz, 1843, 214.

Scylliodus Agassiz, 1843, 214.

Paleoscyllium Wagner, 1857, 276.

Orthodon Coquand, 1860, 295.

Cephaloscyllium Gill, 1861, 306.
Halælurus Gil1, 1861, 306.

Parascyllium Gill, 1861, 306.

Pal aosCyllium von der Marck, 1863, 298.

MESiteia Kramberger, 1885, 433.

Cantioscyllium Woodward, 1889, 449.

Proscyllium Hilgendorf, 1904, 508.

Parmaturus Garman, 1906, 518.

Haploblepharus Garman, 1913, 548.

Apristurus Garman, 1913, 548.

Atelomycterus Garman, 1913, 548.

Catulus (Valmont) Garman, 1913, 548.

\section{Family 53. PENTANCHIDAE}

First dorsal wanting.

Caninoa ${ }^{10}$ Nardo, 1844, 221.

Caninotus Nardo, 1844, 222.
Thalassoklephtes Gistel, 1848, 235.

Pentanchus ${ }^{\mathrm{s0}}$ Smith, 1912, 546.

${ }^{40}$ This genus, which has received three names, is perhaps mythical.

${ }^{\text {so }} \mathrm{This}$ genus, distinguished by the absence of the first dorsal, is regarded by Regan as a mutilated scylliorhinoid; this is an error as a second (undescribed) species, of this family, locally known as Kagurazame, occurs in Sagami Bay.

\section{Family 54. ORECTOLOBID}

Orectolobus Bonaparte, 1837, 187.

Stegostoma Müller \& Henle, 1837, 192, 573.

Crossorhinus M. \& H., 1838, 192.

Synchismus Gill, 1861, 306.
Brachælurus Ogilby, 1906, 519.

Heteroscyllium Regan, 1908, 530.

Cirriscyllium Ogilby, 1908, 529.

Eucrossorhinus Regan, 1908, 530.

TomonoN ${ }^{51}$ Leidy, 1865, 338.

${ }^{61}$ Tomodon = Diplotomodon Leidy, 1868, "Genera of Fishes," 352, is regarded by Dr. Hay as the tooth of a Mosasaur reptile. The name is preoccupied.

\section{Family 55. GINGLYSTOMID $\approx$}

This family is scarcely distinct from the Orectolobidce.

Nebrius Rüppell, 1835, 184.

Ginglymostoma Müller \& Henle, 1837, 190.

${ }^{52}$ Plagiostomia, 56; type Nebrius concolor Rüppell substituted for Nebrius, regarded as preoccupied. (Omitted in "Genera of Fishes.")

Family 56. PSEUDOTRIAKIDE

Pseudotriakis $^{53}$ Capello, 1868, 349.

\footnotetext{
${ }^{*}$ Also written Pseudotriacis.
}

ACroDOBatis Leidy, 1877, 391.
Nebrodes $^{62}$ Garman, 1913. 


\title{
Family 57. RHINEODONTID丑
}

\author{
Rhincodon ${ }^{54}$ Smith, (1849), 174, 244. Micristodus Gill, 1865, 337.
}

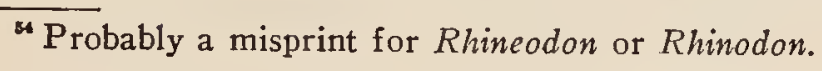

\section{Series LAMNOIDEI}

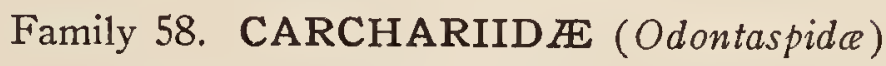

(Sand Sharks)

Carcharias $^{55}$ Rafinesque, 1810, 77.

Odontaspis Agassiz, 1836, 184.

Triglochis Müller \& Henle, 1838, 192.
Oxytes Giebel, 1847, 231.

Eugomphodus Gill, 1864, 331.

XIP HODOLAMIA ${ }^{55 a}$ Leidy, 1877, 301.

${ }^{*}$ The status of the name Carcharias as determined by the International Commission of Zoological Nomenclature makes it identical with Odontaspis, although the obvious intent of Rafinesque was to apply it to the group called Carcharhimus.

${ }^{65}$ This genus, having the teeth with bifid roots, the branches closely appressed, can not belong to the Hexanchida, in which family the teeth all have solid roots.

\section{Family 59. MITSUKURINIDE (Goblin Sharks)}

Rhinognathus Davis, $1885,431$.

Mitsukurina Jordan, 1898, 480.

SCAPANORHYNCHUS Woodward, 1889, 449.

\section{Family 60. LAMNID \\ (Mackerel Sharks; Man-Eaters)}

Isurus Rafinesque, 1810, 78.

Lamna Cuvier, 1817, 97.

Lamia Risso, 1826, 119.

Selanonius Fleming, 1828, 122.

Oxyrhina Ágassiz, 1838, 190, 218.

Corıx Agassiz, 1843, 214.

Sphenodus Agassiz, 1843, 214.

Carcharodon Müller \& Henle, 1838, 190, 192, 574.
Otonus Agassiz, 1843, 214.

Exoles Gistel, 1848, 236.

Plectrostoma Gistel, 1848, 237.

Isuropsis Gill, 1861, 306.

Xenonola mia ${ }^{\text {Bga }}$ Leidy, 1877.

Orthacodus Woodward, 1889, 449.

Pseudocorax Priem, 1897, 478.

Leptostyrax Williston, 1900, 491.

Carcharocles ${ }^{56}$ Jordan \& Hannibal, 1923.

${ }^{s} \mathrm{New}$ genus; orthotype Carcharodon auriculatus (Blainville). Similar to Carcharodon, but with a strong denticle on each side of the base of the tooth. Teeth narrower and more erect than in Carcharodon, their edges finely serrated.

Journ Acad Nat. Sci. Phila., 8: 251; logotype X. pravus Leidy. (Omitted in "Genera of Fishes.")

\section{Family 61. ALOPIID正}

Vulpecula Valmont, 1768, 27.

Alopecias Müller \& Henle, 1838, 192.

Alopias Rafinesque, 1810, 78.

Vulpecula (Valmont) Garman, 1913, 548.

\section{Family 62. CETORHINID压}

\section{(Basking Sharks)}

Tetroras $^{57}$ Rafinesque, 1810, 77.

Cetorhinus Blainville, 1816, 95.

Selache Cuvier, 1817, 97.
Polyprosopus Couch, 1862, 313.

Hannovera Van Beneden, 1871, 360.

${ }^{67}$ A name given to a doubtful or perhaps wholly mythical account apparently of the Basking Shark (Cetorhimus). 


\section{Series GALEOIDEI}

\section{Family 63. GALEIDE (Galeorhinide; Carcharhinida)}

(Gray Sharks)

Galeus Valmont, 1768, 27.

Mustelus Valmont, 1768, 27.

Galeus Klein, 1775, 37.

Cynocephalus Klein, 1777, 42, 407.

Mustelus Linck, 1790, 49.

Aodon $^{58}$ Lacépède, 1798, 55.

Carcharias $^{50}$ Rafinesque, 1810, 77.

Galeus Rafinesque, 1810, 78.

Mustelus Leach, 1812, 84.

Mustellus Fischer, 1813, 85.

Carcharhinus Blainville, 1816, 95.

Galeorhinus Blainville, 1816, 95.

Carcharias Cuvier, 1817, 97.

Galeus Cuvier, 1817, 97.

Mustelus Cuvier, 1817, 97.

Leptocarias Smith, 1837, 190.

Scoliodon Müller \& Henle, 1837, 190.

Galeocerdo M. \& H., 1837, 192, 573.

Leptocharias M. \& H., 1837, 193, 573.

Physodon $^{80}$ M. \& H., 1838.

Aprion M. \& H., 1838, 192.

Hypoprion M. \& H., 1838, 192.

Triakis M. \& H., 1838, 192.

Triznodon M. \& H., 1838, 192.

Loxodon M. \& H., 1838, 192.

Thalassorhinus M. \& H., 1838, 192.

Prionodon M. \& H., 1838, 192.

Hemipristis Agassiz, 1843, 214.

Glyphis Agassiz, 1843, 402.

Myrmillo Gistel, 1848, 237.

Prionace Cantor, 1849, 241.

Hemigaleus Bleeker, 1852, 250.
Galeodes Heckel, 1854, 259.

Microdus Emmons, 1857, 275.

Protogaleus Molin, 1860, 298.

Platypodon Gill, 1861, 305, 331.

Hypoprionodon Gill, 1861, 306.

Eulamia Gill, 1861, 306.

Isogomphodon Gill, 1861, 306.

Lamiopsis Gill, 1861, 306.

Isoplagiodon Gill, 1861, 306.

Aprionodon Gill, 1861, 303, 306.

Cynocephalus (Klein) Gill, 1861, 306.

Boreogaleus Gill, 1861, 306.

Chænogaleus Gill, 1861,'306.

Rhinotriacis Gill, 1862, 317.

Eugaleus Gill, 1864, 330.

Pleuracromylon Gill, 1864, 330.

Alopiopsis $^{61}$ Lioy, 1865, 388.

Leptocarcharias (Smith) Günther, 1870, 357.

Dirrhizodon Klunzinger, 1870, 358.

Pseudogaleus Jaekel, 1894, 465.

Scylliogaleus Boulenger, 1902, 497. Cynias $^{82}$ Gill, 1903.

Hunsruckia Traquair, 1903, 506.

Gemundina Traquair, 1903, 506.

Carcharioldes Ameghino, 1906, 516.

Calliscyllium Tanaka, 1912, 546.

Carcharoides Chapman, 1913, 547.

Eridacnis Smith, 1913, 551.

Rhizoprion Ogilby, 1915, 557.

Gyrace $^{\text {*3 }}$ Jordan \& Hannibal, 1922.

\footnotetext{
${ }^{58}$ Perhaps imaginary.

"As "rigidly construed" the name Carcharias of Rafinesque must be excluded from this family.

"Plagiostomen, 30; orthotype Carcharias mulleri Müller \& Henle. (Omitted in "Genera of Fishes.")

${ }^{11}$ Misprinted Hopiopsis by Zittel.

${ }^{22}$ Proc. U. S. Nat. Mus., 960, 1903; orthotype Squalus canis Mitchill. (Omitted in "Genera of Fishes.")

${ }^{\natural 7}$ Gyrace new genus; orthotype Scymnus occidentalis Agassiz, California Miocene, allied to Galeorhinus but with the longer teeth peculiarly twisted, and coarsely serrated at base.
} 


\section{Family 64. SPHYRNID无}

(Hammer-head Sharks)

This family is hardly separable from the Galcida, differing only in the form of the head.

Cestracion Klein, 1775, 40.

Sphyrna Rafinesque, 1810, 82.

Sphyrnias Rafinesque, 1815, 92.

Cestrorhinus Blainville, 1816, 95.

Zygæna Cuvier, 1817, 97.

Sphyrichthys Thienemann, 1828, 173.

Platysqualus Swainson, 1839, 204.

Sphyra Van der Hoeven, 1849, 243.

Cestracion (Klein) Gill, 1861, 306, 303.

Eusphyra Gill, 1861, 306.

Reniceps Gill, 1861, 306.

Cestracion (Walbaum after Klein)

Ogilby, 1916, 560.

\section{Family 65. PRISTIOPHORID Æ}

(Saw Sharks)

Pristiophorus Müller \& Henle, 1837, 192, 573.

\section{Family 66. PLIOTREMID $A$}

Pliotrema Regan, 1906, 520.

\section{Order TECTOSPONDYLI \\ Suborder SQUALOIDEI \\ Family 67. SQUALID $\mathbb{E}$ \\ (Dog-fishes)}

These sharks which, like the rays, lack the anal fin have also simplified or degenerate vertebræ in which the hard material is arranged in rings without radiation. In the most primitive family, Squalida, spines are present in each of the two dorsal fins, a character which may indicate descent from allies of the Heterodontida, in which group (Cestraciontes) such spines also occur.

Squalus Linnæus, 1758, 11.

Dalatias Rafinesque, 1810, 77.

Cerictius $^{64}$ Rafinesque, 1810, 78.

Etmopterus Rafinesque, 1810, 78.

Acanthorhinus Blainville, 1816, 95.

Spinax Cuvier, 1817, 97.

Acanthias Risso, 1826, 119.

Centroscyllium Müller \& Henle, 1838, 193.

Centrophorus M. \& H., 1838, 192.

Acanthidium Lowe, 1839, 195.

Spinax Bonaparte, 1845, 225.

Lepidorhinus Bonaparte, 1845, 225.

Acanthias Bonaparte, 1845, 225.

Carcharias Gistel, 1848, 234.
Centropterus Costa, 1861, 301.

Entoxychirus Gill, 1862, 317.

Scymnodon Bocage \& Capello, 1864, 329.

Centroscymnus B. \& C., 1864, 329.

Machephilus Johnson, 1867, 346.

Centrophoroides Davis, 1885, 431.

Paracentroscyllium Alcock, 1889, 444.

Deania Jordan \& Snyder, 1902, 499.

Zameus Jordan \& Fowler, 1903, 504.

Nasisqualus Smith, 1912, 546.

Centroselachus $^{65}$ Garman, 1913.

Protospinax Woodward, 1918, 565.

Atractophorus Gilchrist, 1922. (See index.)

${ }^{64}$ Mythical.

${ }^{85}$ Plagiostomia, 306; orthotype Centrophorus crepidater Bocage and Capello. (Omitted in "Genera of Fishes.") 


\section{Family 68. OXYNOTID压}

Oxynotus Rafinesque, $1810,82$.

Centrina Cuvier, 1817, 97.

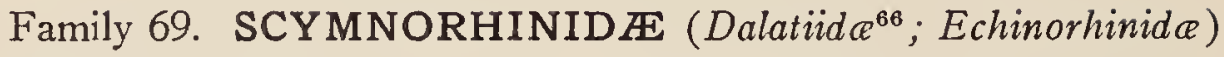

Echinorhinus Blainville, 1816, 95.

Scymnus Cuvier, 1817, 97.

Goniodus Agassiz, 1836, 184.

Scymnorhinus Bonaparte, 1845, 225.

Borborodes Gistel, 1848, 237.
Euprotomicrus ${ }^{67}$ Gill, 1864, 331.

Isistius Gill, 1864, 331.

Leius Kner, 1865, 338.

Cirrhigaleus Tanaka, 1912, 546.

Squaliolus Smith, 1912, 546.

${ }^{*}$ The earliest logotype of Dalatias Rafinesque was one of the Squalida, possibly Centrophorus granulosus.

or This genus and the next two are very aberrant, and each might stand as type of a distinct family. The same is also true of Echinorhinus.

\section{Family 70. SOMNIOSID瓜}

\section{(Sleeper Sharks)}

Somniosus LeSueur, 1818, 107.

Læmargus Müller \& Henle, 1838, 192.

Leiodon Wood, 1847, 232.
Rhinoscymnus Gill, 1864, 331.

Heteroscymnus Tanaka, 1912, 546.

\section{Suborder SQUATINOIDEI}

\section{Family 71. SQUATINIDÆ}

(Angel Sharks)

Rhina Klein, 1775, 39.

Squatina Duméril, 1906, 75.

Rhina Rafinesque, 1810, 78.

Squalraia De la Pylaie, 1835, 183.

Thaumas Münster, 1842, 212.
Phorcynus Thiollière, 1854, 261.

Rhina (Klein) Gill, 1861, 306.

Scaldia Le Hon, $1871,361$.

Trigonodus Winkler, 1878, 398.

\section{Order BATOIDEI}

(Hypotremata)

Suborder SARCURA

Family 72. PRISTID在

Pristis Klein, 1775, 41.

Pristis Linck, 1790, 49.

Pristis Latham, 1794, 52.

Pristibatis Serville, 1829, 134.

Myriosteon Gray, 1864, 332.

Propristis Dames, 1883, 422.
(Saw-fishes)

Amblypristis Dames, 1888, 440.

SClerorhy NChus Woodward, 1889, 449.

Pristiopsis Fowler, 1905, 512.

Eopristis Stromer, 1905, 516.

ONYChOPRIstis ${ }^{88}$ Stromer, 1918.

${ }^{\circ}$ Abhandl. Bayer. Akad., 28: 17; orthotype Gigantichthys mumidus Haug.

Family' 73. RHINIDEE (Rhamphobatida; Rhynchobatide)

Rhina Bloch \& Schneider, 1801, 59.

Rhamphobatis Gill, 1861, 306.

Rhynchobatus $^{\circ}$ Müller \& Henle, 1837, 192, 573.

\footnotetext{
Sometimes spelled Rhynchobatis. The above is the original form.
} 


\section{Family 74. RHINOBATID正}

(Guitar-fishes)

Rhinobatos Klein, 1775, 40.

Rhinobatus Linck, 1790, 49.

Rhinobatus Bloch \& Schneider, 1801, 59.

Leiobatus Rafinesque, 1810, 78.

Squatinoraja Nardo, 1824, 116.

Aellopos Münster, 1836, 185.

Syrrhina Müller \& Henle, 1838, 192.

Trygonorrhina $^{70}$ M. \& H., 1838, 192.
Euryarthra Agassiz, 1843, 214.

Cyclarthrus Agassiz, 1843, 214.

Glaucostegus Bonaparte, 1845, 225.

Demiurga Gistel, 1848, 237.

Spathobatis Thiollière, 1849, 244, 261.

Rhynchobatis Philippi, 1857, 276.

Zapteryx Jordan \& Gilbert, 1880, 404.

Tarșistes Jordan, 1919, 567.

${ }^{70}$ This name appears first in Ann. Mag. Nat. Hist. (2), 2: 90, 1838, spelled with " $r$."

\section{Family 75. ASTERODERMID $\mathbb{E}$}

Asterodermus ${ }^{71}$ Agassiz, 1843, 214.

Belemnobatis Thiollière, 1854, 261. 1829.

"Asterodermus Agassiz, 1843, is perhaps preoccupied by Astrodermus Bonelli,

Family 76. PLATYRHINID $\#$ (Discobatide; Analithide)

Platyrhina Müller \& Henle, 1838, 193.

Analithis Gistel, 1848, 237.

Discobatus Garman, 1880, 402.

Platyrhinoidis Garman, 1880, 402 .

Zanobatus Garman, 1913, 548.

Arhynchobatis Waite, 1909, 534.

\section{Family 77. RAJID $Æ$}

(Rays; Skates)

Raja $^{72}$ Linnæus, $1758,11$.

Leiobatus Klein, 1775, 38.

Dipturus Rafinesque, 1810, 78.

Cephaleutherus Rafinesque, 1810, 83.

Platopterus Rafinesque, 1815, 92.

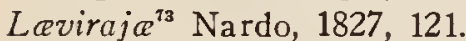

Propterygia Otto, 1824, 117.

Rajabatis De La Pylaie, 1835, 183.

Batis Bonaparte, 1837, 187.

Læviraja Bonaparte, 1837, 187.

Dasybatus Bonaparte, 1837, 187.

Sympterygia Müller \& Henle, 1838, 193.
Uraptera M. \& H., 1838, 193.

Hieroptera Fleming, 1841, 209.

Actinobatis Agassiz, 1843, 214.

Psammobatis Günther, 1870, 357.

Oncobatis Leidy, $1871,362$.

Leucoraja Malm, 1877, 391.

Amblyraja Malm, 1877, 391.

Malacorhina Garman, 1877, 389.

Acanthobatis Larrazet, 1886, 436.

Platyspondylus Haug, 1905, 514.

Malacobatis Gracianov, 1907, 523.

Dactylobatus Bean \& Weed, 1909, 531.

${ }^{72}$ Also spelled Raia by authors.

${ }^{73}$ Name given in plural form only.

\section{Family 78. ARTHROPTERIDÆ}

Arthropterus Agassiz, 1843, 214.

\section{Suborder NARCACIONTES}

Family 79. TORPEDINID $Æ$ (Narcaciontida; Narcobatida)

(Torpedos; Numb-fishes)

Torpedo ${ }^{74}$ Forskål, 1775, 32.

Narcacion Klein, 1775, 39.
Torpedo Duméril, 1806, 75.

Torpedo Rafinesque, 1810, 82. 
Narcobatus Blainville, 1816, 95.

Narke Kaup, 1826, 121.

Temera Gray, 1831, 138.

Narcine Henle, 1834, 180.

Astrape Müller \& Henle, 1838, 193.

Syrraxis (Jourdan) Bonaparte, 1841, 208.

Narcopterus Agassiz, 1843, 214.

Discopyge Tochudi, 1846, 230.

Hypnos Duméril, 1852, 250.

Narcacion (Klein) Bleeker, 1866, 340.

Gonionarce Gi1l, 1861, 307.
Cyclonarce Gill, 1861, 307.

Tetranarce $^{75}$ Gill, 1861, 307.

Fimbriotorpedo Fritsch, 1886, 435.

Gymnotorpedo Fritsch, 1886, 435.

Benthobatis Alcock, 1898, 478.

Hypnarce Waite, 1902, 501.

Tetronarcine Tanaka, 1908, 531.

Bengalichthys Annandale, 1909, 531.

Typhlonarke Waite, 1909, 534.

Eunarce Fowler, 1910, 535.

Heteronarce $^{75 a}$ Regan, 1921.

${ }^{74}$ In part; the name is used in an oblique case only, and the Electric Ray is confused with the Electric Cat-fish.

${ }^{75}$ Misprinted Tetronarce.

ive Orthotype $H$. garmani Regan.

\section{Suborder MASTICURA \\ (Centrobaies)}

Family 80. DASYATID $¥$ (Trygonida; Dasybatida)

(Sting-Rays)

Dasybatus Klein, 1775, 39.

Dasyatis Rafinesque, 1810, 78.

Uroxis Rafinesque, 1810, 83.

Trygonobatus Blainville, 1816, 94.

Leiobatus Blainville, 1816, 95.

Trygon (Adanson) Cuvier, 1817, 98.

Trygon Isidore Geoff roy St. Hilaire, 1826, 120.

Pastinace ${ }^{76}$ Nardo, 1827, 121.

Pastinachus Rüppel1, 1828, 122.

Anacanthus (Ehrenberg) Cuvier, 1829, 131.

Tæniura Müller \& Henle, 1837, 189.

Himantura M. \& H., 1837, 190.

Gymnura M. \& H., 1837, 190.

Urogymnus M. \& H., 18.37, 190.

Hemitrygon M. \& H., 1837, 190.

Pteroplatea M. \& H., 1838, 193.

Trygonoptera M. \& H., 1838, 193.

Urolophus M. \& H., 1838, 193.

Hyplophus M. \& H., 1838, 193.
Pastinaca Dekay, 1842, 210.

Cyclobatis Egerton, 1844, 220.

Rhachinotus Cantor, 1849, 241.

Pastinaca Gronow, 1854, 258.

Pastinaca Duméril, 1856, 268.

Alexandrinus Molin, 1861, 308.

Ưrotrygon Gill, 1863, 324.

Neotrygon Castelnau, 1873, 368.

Gryphodobatis Leidy, 1877, 391.

Heliobatrs Marsh, 1877, 391.

Xiphotrygon Cope, 1879, 399.

Dasybatus (Klein) Garman, 1885, 432.

Discobatis Miklukho-Maclay \& Macleay, 1886, 436.

Discotrygon Fowler, 1910, 535.

Pteroplatytrygon Fowler, 1910, 535.

Hypolophrtes Leriche, 1913, 549.

Amphotistius ${ }^{77}$ Garman, 1913.

Urobatis Garman, 1913, 548.

Dasybatus (Klein, Walbaum) Ogilby, 1916, 560 .

${ }^{78}$ Name given in plural form only.

"Plagiostomia, 8; type Trygon sabina Le Sueur. (Omitted in "Grenera of Fishes.")

\section{Family 81. POTAMOTRYGONIDÆ (Elipesuride)}

Elipesurus Schomburgk, 1842, 212.

Paratrygon Duméril, 1865, 337.

Dynatobatis Larrazet, 1886, 436.

Disceus Garman, 1877, 389.

Potamotrygon Garman, 1877, 389. 


\section{Family 82. BRACHIOPTERID $\mathbb{E}$}

Brachioptera Gracianov, 1906, 518.

Phanerocephalus $^{78}$ Gracianov, 1906, 518.

${ }^{78}$ Misprinted Planerocephalus.

\section{Family 83. PTYCHODONTID瓜}

Pтrсhodus Agassiz, 1839, 194.

Hemirtychodus Jaekel, 1894, 465.

Sporetodus Cope, 1874, 375.

\section{Family 84. MYLIOBATID正 (Ä̈tobatida)}

(Eagle Rays)

Aëtobatus $^{70}$ Blainville, 1816, 95.

Goniobatis Le Hon, 1871, 361.

Myliobatis (Duméril) Cuvier, 1817, 573.

Aëtoplatea Müller \& Henle, 1838, 193.

Mesobatis Leidy, 1877, 391.

Ptychopleurus Agassiz, 1838, 190.

Bates Probst, 1877, 392.

Stoasodon Cantor, 1849, 241.

Aulodus Dixon, 1850, 245.

Goniobatis Agassiz, 1858, 277.

Holorhinus Gill, 1862, 317.

Plintuicus Cope, 1869, 354.

Apocoponon Cope, 1886, 434.

Promyliobatis Jaekel, 1894, 465.

Aëtomylæus Garman, 1908, 528.

Pteromylæus Garman, 1913, 548.

HyL.eobatis Woodward, 1915, 558.

${ }^{78}$ Also written Aëtobatis.

\section{Family 85. RHINOPTERID卌}

Rhinoptera (Kuhl) Cuvier, 1829, 173.

Zygobatis Agassiz, 1836, 184, 214.

Mylorhina Gill, 1865, 337.

Micromesus Gill, 1865, 337.

Family 86. MOBULID王 (Mantide; Cephalopteride)

(Devil Rays)

Cephalopterus Risso, 1810, 77.

Mobula Rafinesque, 1810, 82.

Apterurus Rafinesque, 1810, 83.

Dicerobatus Blainville, 1816, 95.

Manta Bancroft, 1829, 174, 573.
Ceratoptera Müller \& Henle, 1838, 193.

Pterocephala Swainson, 1839, 204.

Brachioptilon Newman, 1849, 244.

Diabolichthys Holmes, 1856, 270.

Ceratobatis Boulenger, 1897, 476.

\section{Subclass HOLOCEPHALI \\ Order CHIMAEROIDEI \\ (Chismopnea) (Chimæras)}

\section{Family 87. PTYCTODONTID卌}

Aulacosteus Eichwald, 1846 (1860), 295, Rhynchodus Newberry, 1873, 371. 229.

Ramphodus Jaekel, 1903, 504.

Ptyctodus Pander, 1858, 283.

Rinodus Newberry \&. Worthen, 1866, 342.

ACMONIODUs Hussakof \& Bryant, 1919, 566.

Deinodus H. \& B., 1919, 566.

\section{Family 88. SQUALORAIID $\not E$}

SQUaloraia Riley, 1826, 173.

Chalcodus Zittel, 1887, 439.

Spinacorhinus Agassiz, 1836, 184. 


\section{Family 89. MYRIACANTHIDE}

Ctenacanthus ${ }^{80}$ Agassiz, 1836, 186.

Myriacanthus Agassiz, 1837, 186.

Physonemus ${ }^{80}$ M'Coy, 1848, 239.

Xystracanthus ${ }^{80}$ Leidy, 1859, 292.

Drepanacanthus ${ }^{80}$ Newberry \&

Worthen, 1866, 342.

Prognathodus Egerton, 1872, 365.
Batacanthus ${ }^{80}$ St. John \& Worthen, $1883,425$.

Acond ylacanthus St. J. \& W., 1883, 425.

Metopacanthus Zittel, 1887, 439.

Снiм жеropsis Zittel, 1887, 439.

${ }^{80}$ Probably all these represent frontal claspers of a chimæroid; see Eastman, Proc. U. S. Nat. Mus., 52: 262, 1917.

\section{Family 90. CHIMÆRID压 \\ (Chimaras or Elephant-fishes)}

Chimæra Linnæus, 1758, 11.

Leptacanthus Agassiz, 1837, 186.

Oracanthus Agassiz, 1837, 186.

EDAPHODOn Buckland, 1838, 191.

PASSALODON Buckland, 1838, 191.

Ameibodon Buckland, 1838, 191.

Ischyodus Egerton, 1843, 214.

Elasmodus Egerton, 1843, 214.

Gaxodus Agassiz, 1843, 214.

Psittacodon Agassiz, 1843, 214.

Erismacanthus ${ }^{81}$ M'Coy, 1848, 239.

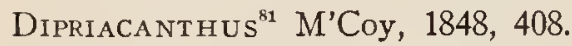

Mylognathus Leidy, 1856, 272.

Chim.eracanthus Quenstedt, 1858, 283.

Hydrolagus Gill, 1862, 317.

Auluxacanthus Sauvage, 1867, 347.

Leptomylus Cope, 1869, 354.

Dipristis Gervais, 1869, 504.

Sphagepcea Cope, 1870, 356.

Dipristis Marsh, 1870, 359.

Eumylonus Leidy, $1873,370$.

LeCracanthus ${ }^{81}$ St. John \& Worthen, $1875,381$.

GampsaCANthus ${ }^{81}$ St. J. \& W., 1875, 381.
Isotenia Cope, $1875,379$.

Diphrissa Cope, 1875, 379.

Bryactinus Cope, $1875,379$.

Hedronchus Cope, 1876, 385.

Myledaphus Cope, 1876, 385.

Elasmognathus Newton, 1878, 397.

Cladacanthus (Agassiz) Davis, (1842) $1883,213,422$.

Harpacanthus Traquair, 1886, 436.

Elasmodectes Newton, 1888, 444

Stethacanthus Newberry, 1889, 447.

$V_{\text {AILlantoönIA }}{ }^{82}$ Meunier, 1891, 455.

Palcchimara $^{83}$ Walcott, 1891, 456.

Elasmodectes Newton, 1891, 456.

PALÆOM YLUS Woodward, 1891, 456.

Dictyorhabdus ${ }^{83}$ Walcott, 1892, 459.

PACHYMylus Woodward, 1892, 460.

BRACH Y M YLUS Woodward, 1892, 460.

AMyLodon Storms, 1894, 466.

BelemnaCanthus Eastman, 1898, 479.

Bathyalopex Collett, 1901, 492.

Psychichthys Fowler, 1907, 523.

Acanthorhina Fraas, 1910, 535.

${ }^{81}$ These four nominal genera are regarded by Eastman as identical and based on frontal claspers of male chimæroids.

${ }^{82}$ Fossil egg-case of a chimæroid.

${ }^{83}$ It seems by no means certain that Dictyorhabdus (Palcechimara) is really a fish relic. Occurring in the Ordovician, it is far older than any known Chimara. It may be a Graptolite. Cockerell informs me that he "has good material showing surface sculpture and is entirely convinced that it is not a vertebrate." 
Note on Ichthyodorulites: The fin-spines named below may have belonged to species of Chimarida or of some related family.

Cylindracanthus Leidy, 1856, 273.

Rabdacanthus Pander, 1856, 274.

Byssacanthus Salter, 1861, 309.

Listracantiuus Newberry \& Worthen, $1870,359$.

Cyrtacantuus Newberry, 1873, 371.
Ostracacanthus Davis, 1879. 399.

Euctenius Traquair, 1881, 417.

Euncanthus Trautschold, 1883, 426.

Gnathacanthus Davis, 1883, 422.

Apateacanthus Woodward, 1891, 456.

Glyptorhynchus Leriche, 1905, 515.

\section{Family 91. RHINOCHIMERIDAE}

Harriotta Goode \& Bean, 1894, 464.

Anteliochimæra Tanaka, 1909, 534.

Rhinochimæra Garman, 1901, 493.

Family 92. CALLORHYNCHIDE

Callorhynchus Gronow, 1763, 18.

Callorhynchus (Gronow) Cuv., 1817, 98. 


\section{Class PISCES}

\section{(True Fishes) \\ Subclass CROSSOPTER YGII}

The fishes of this group possess a jointed pectoral limb which may be homologous with that of the primitive sharks of the order Ichthyotomi. It is not certain, however, that this is not a case of analogy, in which case it does not indicate genetic relationship. The class is certainly very old in geologic time, standing at the base of the series of true fishes, while at the same time holding an ancestral relation to the amphibia and the higher vertebrates. A few species are still extant.

\section{Order RHIPIDISTIA}

(Taxistia)

\section{Family 93. HOLOPTYCHID E}

Holoptychus ${ }^{84}$ Agassiz, 1839, 194.

Dendrodus Owen, 1841, 209.

Platygnathus Agassiz, 1844, 218.

GLy PTOLEPIS Agassiz, 1844, 218.

Sclerolepis Eichwald, $1844,220$.
Lam nodus Agassiz, 1845, 225.

ApEnodus Leidy, 1858, 283.

ERIPTychius $^{85}$ Walcott, 1892, 459.

Mawsonia Woodward, 1907, 525.

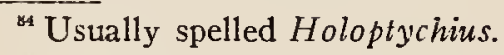

${ }^{85}$ Scales of the Ordovician age, doubtful as to interpretation.

Family 94. MEGALICHTHYID $\mathbb{E}^{86}$ (Rhizodontida)

Megalichthys Agassiz \& Hilbert, 1836, Gastrodus Owen, 1867, 347. 185.

Rhizodus Owen, 1840, 207.

Criconus Agassiz, 1844, 217.

SAURIPTERIS $^{87}$ Hall, 1843, 214.

Colonodus M'Coy, 1848, 239.

Gyroptycinius M'Coy, 1848, 239.

Rabdiolepis Emmons, 1857, 275.

Polyplacodus Pander, 1860, 298.

TRISTICHOPTERus Egerton, 1861, 301.

Strepsodus Young, 1866, 344.

RHIzoDopsis Young, 1866, 344.

Gastrodus Owen, $1867,347$.

GaNolodus Owen, 1867, 347.

Archichthys Hancock \& Atthey, 1870, 358.

LABYRINTHODONTOSAURUS Barkas, 1873, 366.

Orthognathus Barkas, 1873, 366.

Sigmodus $^{\text {87a }}$ Waagen, 1879.

Eusthenopteron ${ }^{88}$ Whiteaves, 1881, 418.

Cerosteus ${ }^{89}$ Newberry, 1887.

Dictyonosteus ${ }^{30}$ Stromer, 1918.

Dendroptychius (Huxley) Young, 1866, 344.

${ }^{s e}$ The name Megalichthys has priority over its synonym Rhizodus.

${ }^{87}$ Usually written Sauripterus.

8: Loc. cit., 10; orthotype S. dubius Waagen. (Omitted in "Genera of Fishes.")

${ }^{8}$ Misprinted Eusthenopleuron in "Genera of Fishes."

${ }^{60}$ Trans. N. Y. Ac. Sci, 6: 137; type C. ferox Newberry. (Omitted in "Genera of Fishes."

${ }^{\infty}$ Bull. Geol. Soc. Upsala, 16: 116; orthotype D. arcticus Stromer. 
Family 95. OSTEOLEPID王 (Rhombodipterida)

Osteolepis Valenciennes, 1829, 134.

Diplopterus Agassiz, 1835, 181.

Megalichthys Agassiz, 1844, 217.

Glyptopomus Agassiz, 1844, 218.

Tripterus M'Coy, 1848, 239.

Parabatrachus Owen, 1853, 254.

Diplopterax M'Coy, 1855, 265.

Triplopterus, M'Coy, 1855, 266.

Gyrolepis Kade, 1858, 281.

Gr.yp'Tol emus (Huxley) Anderson, 1859, 287.
Sporolepis Romanowsky, 1864, 334.

Plintholepis Romanowsky, 1864, 334.

RномвортусіIUs (Huxley) Young, 1866, 344.

Ectosteoriachis Cope, $1880,402,422$.

Thursius Traquair, 1888, 443.

Glyptognathus Woodward, 1891, 456.

Porolepis Woodward, 1891, 456.

Gyropeltus Rohon, 1893, 462.

PAleosteus Rohon, 1893, 462.

\section{Family 96. ONYCHODONTIDAE}

Onychodus Newberry, 1857, 276.

\section{Order ACTINISTIA} (Colacanthini)

\section{Family 97. CEELACANTHIDA}

Undina Münster, 1834, 180.

Maсropoma Agassiz, 1835, 181, 280.

Libys Münster, 1842, 212.

Celacanthus Agassiz, 1844, 218.

Hoplopygus Agassiz, 1844, 217.

Heptanema Bellotti, 1857, 274.

KoKkonerma ${ }^{\text {91 }}$ Quenstedt, 1858, 283.

Graphiurus Kner, 1865, 341.

LOPHOPRIONOLEPIS Steinla, 1868, 350.

Peplorhina Cope, 1873, 368.

Conchiopsis Cope, 1873, 368.
Diplurus Newberry, 1878, 396.

R.habDonerma Reis, 1888, 443.

CelaCANThopsis Traquair, 1901, 576.

LeIoner Ma ${ }^{22}$ Stensiö, 1918.

Wimania ${ }^{93}$ Stensiö, 1921.

SASSENIA $^{94}$ Stensiö, 1921.

Axelia ${ }^{\text {B5 }}$ Stensiö, 1921.

Mylacanthus ${ }^{20}$ Stensiö, 1921.

Scleracanthus ${ }^{97}$ Stensiö, 1921.

Diplocercides $^{\text {98 }}$ (Jaekel) Stensiö, 1922.

"Later corrected to Coccoderma.

${ }^{n}$ Erik A. Son Stensiö, "Zur Kenntniss des Devons und des Kulms an der Klaas, Billenbag Spitzbergen," Geol. Inst. Bull. Upsala, 16; orthotype Leioderma sinuosa Stensiö; name preoccupied, replaced by Wimania Stensiö, 1921.

28 "Triassic Fishes from Spitzbergen," p. 51; orthotype Leioderma sinwosa Stensiö.

"Loc. cit., p. 84; orthotype Sassenia tuberculata Stensiö.

${ }^{25}$ Loc. cit., p. 89; orthotype Axelia robusta Stensiö.

${ }^{n}$ Loc. cit., p. 107; orthotype Mylacanthus lobatus Stensiö.

${ }^{" L}$ Loc. cit., p. 111 ; orthotype Scleracanthus asper Stensiö.

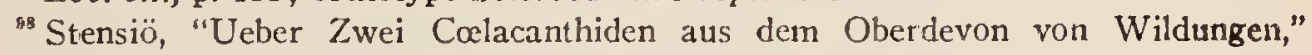
Palcontologisches Zeitschrift, 4: 168; orthotype Holoptychius kayseri von Koenen.

\section{Family 98. TARRASIID 2 E}

TARRASIUS Traquair, 1881, 418.

PAL.EOPHICHTHY'S Eastman, 1908, 527.

Conchopoma Kner, 1868, 352. 


\title{
Order CLADISTIA
}

Family 99. POLYPTERIDE

Polypterus St. Hilaire, 1798, 54.

\section{Family 100. CALAMOICHTHYIDE}

Herpetoichthys (Smith, J. A.) Günther, Erpetoichthys Smith, 1865, 339. $1865,338$.

Calamoichthys Smith, 1866, 343.

\section{Subclass DIPNEUSTA}

(Dipnoi; name preoccupied)

\section{Order CTENODIPTERINI}

\author{
(Ctenodipneusta)
}

Family 101. DIPTERIDE

Dipterus Sedgwick \& Murchison, 1828, Pal adaphus Van Beneden \& De Ko125

Catopterus Agassiz, 1833, 177.

Polyphractus Agassiz, 1844, 218. ninck, 1864, 329.

GOMPHOLEPIS Barrande, 1872, 362.

GANORH YNCHUS Traquair, $1873,371$.

Stagonolepis Agassiz, 1845, 225.

Osteoplax M'Coy, 1848, 239.

Conchodus M'Coy, 1848, 239.

Cheirodus Pander, 1858, 283.

Heliodus Newberry, 1875, 379.

Dipnoites Fritsch, 1888, 441.

TyLodus Rohon, 1893, 462.

Chelomodus Rohon, 1893, 462.

ARCh enNeCtES $^{\text {29 }}$ Von Meyer, 1859.

Pentlandia Watson \& Day, 1916, 561.

"Palcontographica, 7: 12; type A. pertusus Von Meyer. (Omitted in "Genera of Fishes.")

Family 102. PHANEROPLEURID $\mathbb{E}$

Phaneropleuron (Huxley) Anderson, Scaumenacia Traquair, 1893, 463.

$1859,287$.

\section{Order SIRENOIDEI}

\section{Family 103. CTENODONTIDE}

Ctenodus Agassiz, 1838, 191.

Rhadamista Gistel, 1848, 235.

Campylopleuron Huxley, 1867, 346.

SAGENOdus Owen, $1867,347$.
Ptyonodus Cope, 1877, 388.

Megapleuron Gaudry, 1883, 423.

GNATHORHIZA $^{100}$ Cope, $1883,422$.

Hemictenodus Jaekel, 1890, 452.

${ }^{100} \mathrm{~A}$ genus of unoertain relationship.

Family 104. URONEMID $\approx$

Uronemus Agass1z, 1844, $217 . \quad$ Ganopristodus Traquair, 1881, 417.

\section{Family 105. CERATODONTID丑}

\section{(Lung-fishes)}

Ceratodus Agassiz, 1838, 191.

Scropha Gistel, 1848, 235.

Neoceratodus ${ }^{101}$ Castelnau, 1876, 384.

Ompax ${ }^{101}$ Castelnau, 1879, 399.
Gosfordia Woodward, 1890, 453.

Epiceratodus Teller, 1891, 456.

Synthetodus Eastman, 1898, 476.

Metaceratodus Chapman, 1910, 534.

${ }^{101}$ Perhaps mythical, in part at least. 
Family 106. LEPIDOSIRENID $\nsubseteq$

(Mud Sirens)

Lepidosiren Fitzinger, 1837, $189 . \quad$ Amphibichthys Hogg, 1841, 209.

Family 107. PROTOPTERID在

Protopterus Owen, 1839, 196.

Protomelus Hogg, 1841, 209.

Rhinocryptis Peters, 1844, 222.

Subclass ACTINOPTERI

Superorder GANOIDEI

Order CHONDROSTEI

(Lysopteri; Podopterygia; Lyomeri; Heterocerci; Glaniostomi)

Family 108. PALEONISCIDE

Palæoniscum ${ }^{103}$ Blainville, 1818, 108.

Paleothrissum Blainville, 1818, 108.

ACrolepis Agassiz, 1833, 177.

Gyrolepis Agassiz, 1833, 177.

Amblypterus Agassiz, 1833, 177.

Pygopterus Agassiz, 1833, 177.

Cheirolepis Agassiz, 1835, 181.

Coccolepis Agassiz, 1844, 218.

MiCrolepis Eichwald, 1844, 220.

Isodus M'Coy, 1848, 239.

Elonichthys Giebel, 1848, 233.

Urosthenes Dana, 1848, 233.

Trachelacanthus Fischer von Waldheim, 1850, 246.

Propaleoniscus Pomel, 1853, 254.

Oxygnathus Egerton, 1855, 264.

MeColepis Newberry, 1856, 273.

Rhabdolepis Troschel, 1857, 276.

Eurylepis Newberry, 1857, 276.

URoLEPIS Bellotti, 1857, 274.

Cosmolepis Egerton, 1858, 280.

Cen rrolepis Egerton, 1858, 280.

Thrissonotus (Agassiz), (1844) 1858, 217.

MYRIoLEPIS Egerton, 1864, 330.

Cycloptychius Young, 1866, 344.

Ganacrodus Owen, 1867, 347.

Platysiagum Egerton, 1872, 365.

LEIOLEPIS Goldenburg, 1873, 369.

Nematoptychius Traquair, 1875, 382.

Hypterus Owen, 1876, 386.
Sph cerolepis Fritsch, 1877, 389.

ACEntrophorus Traquair, 1877, 392.

Gonatodus Traquair, 1877, 392.

Cosmoptychius Traquair, 1877, 392.

Microconodus Traquair, 1877, 392.

Rhadinichthys Traquair, 1877, 392.

Holurus Traquair, 1881, 418.

CRYPHIOLEPIS Traquair, 1881, 418.

Canobius Traquair, 1881, 418.

Eupalæoniscus Rzehak, 1881, 417.

ANAGLYPHUS Rzehak, 1881, 417.

ACtinophorus Newberry, 1889, 447.

Atherstonia Woodward, 1889, 449.

Apateolepis Woodward, 1890, 453.

Drydenius Traquair, 1890, 453.

Mesopoma Traquair, 1890, 453.

Paleoniscinotus Rohon, 1890, 452.

Edua Sauvage, 1891, 456.

ARCH ÆONISCus Sauvage, 1891, 456.

Tegeolepis Miller, 1892, 458.

HAPLOLEPIS Miller, 1892, 458.

TRISSOLEPIS Fritsch, 1893, 460.

Ganolepis Woodward, 1893, 463.

Pyritocephalus Fritsch, 1894, 464.

SCEleptophorus Fritsch, 1894, 464.

Microconodus Traquair, 1895, 470.

FARNellia Traquair, 1898, 484.

Psilichthys ${ }^{100}$ Hall, 1900.

Schizospondylus Fritsch \& Bayer, 1902, 498.

Diphyodus Lambe, $1902,500$.

${ }^{102}$ Written by Agassiz Palaoniscus.

${ }^{108}$ Thomas Sergeant Hall, "A New Genus and a New Species of Fish from the Mesozoic Rocks of Victoria," Mem. Royal Soc. Victoria, New Series, 12: 121. Triassic, Melbourne. 
ElpISOPHOLIs Woodward, 1908, 531.

Helichthys Broom, 1909, 532.

Hydropessum Broom, 1909, 532.

Pelichthys Broom, 1913, 547.

Disichтнуs Broom, 1913, 547.
Caruichthys Broom, 1913, 546.

Birgeria $^{104}$ Stensiö, 1921.

Boreosomus ${ }^{105}$ Stensiö, 1921.

GLAUCOLEPIS ${ }^{16 \mathrm{~A}}$ Stensiö, 1921.

ACrorhabdus ${ }^{107}$ Stensiö, 1921.

${ }^{104}$ "Triassic Fishes, Spitzbergen," p. 151, 1921; orthotype Saurichthys mougeot Agassiz.

${ }^{108}$ Loc. cit., p. 211; orthotype Acrolepis arcticus Woodward.

${ }^{206}$ Loc. cit., p. 200; orthotype Glaucolepis gyrolepidoides Stensiö.

${ }^{107}$ Loc. cit., p. 220 ; orthotype Acrorhabdus berteli Stensiö.

\section{Family 109. PLATYSOMIDEE (Scrotide)}

Eurynotus Agassiz, 1835, 181.

Platysomus Agassiz, 1835, 181.

Globulodus Münster, 1842, 212.

Cheirodus M'Coy, 1848, 239, 408.

Strotes Gistel, 1848, 237.

Notacmon Gistel, 1848, 236.

Plectrolepis (Agassiz) Egerton, (1835) 1850, 181, 218, 246.

TurSEODUS Leidy, 1858, 283.

Mesolepis Young, 1866, 344.

EURY SOMUS ${ }^{108}$ Young, 1866, 344.
Amphicentrum Young, 1866, 344.

Wardichthys Traquair, $1875,382$.

Benedenius Traquair, 1878, 396.

Phanerosteon Traquair, 1881, 418.

Cheirodopsis Traquair, 1881, 418.

Hemiclanodus Davis, 1884, 427.

Benedenichthys Traquair, 1890, 453.

UROPTERYX (Agassiz) Woodward, 1891, 457.

Pododus Agassiz, 1901, 217.

ECrinesomus Woodward, 1910, 537.

${ }^{106}$ Misprinted Euryosomus in "Genera of Fishes."

\section{Family 110. DORYPTERID死}

Dorypterus Münster, 1842, 212.

\section{Family 111. DICTYOPYGID $\mathbb{E}$ (Catopterida)}

Catopterus Redfield, 1837, 190.

Dictyopyge Egerton, 1847, 231.

Redfieldius Hay, 1899, 486.

Perleidus $^{109}$ de Alessandri, 1910.

${ }^{100}$ Gulio de Alessandri, "Studii sui pesci triasici della Lombardia," Mem. Soc, Ital. Sci. Nat. Mus. Civico Stor. Nat. Milano, 7: 49; orthotype Semionotus altolepis Deecke.

\section{Family 112. ASTERODONTIDE (Colobodontida)}

Asterodon Münster, 1841, 209.

Colobodus Agassiz, 1844, 217.

Tholonus Meyer, 1846, 229, 249.

Omphalodus Meyer, 1847, 322.

Cenchrodus Meyer, 1847, 407.

Hemilopas Meyer, 1847, 408.

Nephrotus Meyer, 1851, 249.
Charitodon ${ }^{108 a}$ Schmid, 1861.

Eupleurodus Gürich, 1884, 428.

Dactylolepis Kunisch, $1885,433$.

Crenilepis Dames, 1888, 440.

Dollopterus Abel, 1906, 516.

Meridensia ${ }^{110}$ Andersson, 1916.

Paralepinotus ${ }^{111}$ Stolley, 1920. Fishes.")

${ }^{1092}$ Loc. cit., 30; orthotype C. glabridens Schmid. (Omitted in "Genera of

${ }^{110}$ Erik Andersson, "Ueber Einige Triasfische aus der Cava Trefontane, Tessin,"

Bull. Geol. Inst. Upsala, 15: 25; orthotype Pholidophorus meridensis de Alessandri.

${ }^{101}$ Ernst Stolley, "Beitrage zur Kenntnis des deutschen Muschelkalken," Palconto. graphica, 63: 41; orthotype Colobodus ornatus Agassiz. 
Family 113. BELONORHYNCHID王

Saurichthys Agassiz, 1834, 179.

STYLORHYNCH Us ${ }^{13}$ Martin, 1873.

IсHTH YORHYNCHUS Bellotti, 1857, 274.

BELONORHYNCHUS Bronn, 1858, 279.
Gufronus Costa, 1862, 312.

Browneichthys Woodward, 1889, 449.

SaURORHYNCHUS ${ }^{118}$ (Münster) Reis, 1891, 207.

119 Carl Martin, "Ein Beitrage zur Kenntniss fossiler Euganoiden," Zeitsch. Deutsche Geol. Gesellsch., 25 : 725; type S. tenuirostris Martin. (Omitted in "Genera of Fishes.")

11 Name only in 1840, defined by Reis in 1891 ; Teleosaurus, p. 346, is a genus of reptiles.

\section{Family 114. DIPHYODONTID丑}

Diphyodus Lambe, 1902, 500.

Stenopropoma Hay, 1903, 503.

\section{Family 115. CHONDROSTEID开}

Chondrosteus (Agassiz) Egerton, (1844) $1858,217,280$.
Groosteus (Agassiz) Egerton, (1844), $1858,217,280$.

\section{Order GLANIOSTOMI}

\section{Family 116. ACIPENSERID平}

(Sturgeons)

Acipenser Linnæus, 1758, 11.

Ichthyocolla Geoffroy, 1767,.24.

Sterleta Güldenstädt, 1772, 168.

Sturio Rafinesque, 1810, 82.

Dinectus $^{114}$ Rafinesque, 1818, 108.

Sterletus Rafinesque, 1820, 113.

Sterletus Brandt \& Ratzeburg, 1833, 178.

Helops B. \& R., 1833, 178.

Huso B. \& R., 1833, 177.

Scaphirhynchus Müller, 1835, 182.

Sturio Müller, 1835, 182.

Antaceus Heckel \& Fitzinger, 1836, 185.
Scaphirhynchus Heckel, 1836, 185.

Lioniscus H. \& F., 1836, 185.

Sterledus Bonaparte, 1845, 225.

Ellops Gistel, 1848, 236.

Scaphirhynchops Gill, 1863, 323.

Schipa Brandt, 1869, 354.

Kessleria Boghdanov, 1882, 418.

Pseudoscaphirhynchus Nikolskii, 1900. 490.

Parascaphirhynchus Forbes \& Richardson, 1905, 512.

Hemiscaphirhynchus Berg, 1912, 542.

\section{Order SELACHOSTOMI}

\section{Family 117. POLYODONTID在}

(Paddle-fishes)

Polyodon Lacépède, 1797, 54.

Polyodon Bloch \& Schneider, 1801, 59.

Spatularia Shaw, 1804, 74.

Platirostra Le Sueur, 1818, 107.
Proceros $^{115}$ Rafinesque, 1820, 113.

Crossopholis ${ }^{1164}$ Cope, 1866, 421, 434.

Psephurus Günther, 1873, 369.

Pholmurus Woodward, 1889, 449.

\footnotetext{
${ }^{116}$ A mythical sturgeon.
}

${ }^{11 s}$ Mythical.

${ }^{115 a}$ Misprinted Crossophilus in "Genera of Fishes," p. 434. 


\section{Order PYCNODONTI}

\section{Family 118. PYCNODONTID在}

PaL.ÆOBalistum Blainville, 1818,108

Pycnodus Agassiz, 1833, 177.

Gyrodus Agassiz, 1833, 177.

Microdon Agassiz, 1833, 177.

Scaphodus Agassiz, 1844, 217, 453.

Gyronchus Agassiz, 1844, 217.

Acrotemnus Agassiz, 1844, 217.

Periodus Agassiz, 1844, 217.

Proscinetes Gistel, 1848, 237.

Coccodus Pictet, 1850, 247.

Mesodon Wagner, 1851, 250.

Ancistrodon Derby, 1852, 251.

Glossodus Costa, 1853, 253.

Cálodus Heckel, 1856, 270.

Stemmatodus Heckel, 1856, $270,409$.

Anomiophthalmus Costa, 1856, 268.
HADRODUs $^{117}$ Leidy, 1858, 283.

TyPODUS ${ }^{118}$ Quenstedt, 1858, 283.

Mesturus Wagner, 1862, 321.

Phaconus Cope, 1869, 354.

Cromm yodus Cope 1870,356 .

Ellipsodus Cornuel, 1877, 389.

Uranoplosus Sauvage, 1879, 401.

Cosmodus Sauvage, 1879, 401.

Athrodon Sauvage, 1880, 405.

XeNopholis Davis, 1885, 431.

Anom ceodus Forir, 1887, 437.

Grypodon Hay, 1899, 486.

Polypsephis Hay, 1899, 486.

Macromesodon Blake, 1905, 576.

EoMesodon Woodward, 1918, 565. is earlier.

${ }^{117}$ Perhaps the tooth of a reptile.

${ }^{118}$ This can not replace the preoccupied name Mesodon, as Typodus Meyer, 1847,

Family 119. SEMIONOTIDIE (Lepidotida; Stylodontida; Dapediida; Sphcrodontida)

DAPEDIU ${ }^{119}$ Leach, 1822, 115.

Tetragonolepis Brown, 1830, 136, 177.

Semionotus Agassiz, 1832, 140.

LePIDOTES ${ }^{120}$ Agassiz, 1832, 141.

LePidosaurus Von Meyer, 1832, 141.

Sph cerodus Agassiz, 1833, 177.

Ambly URUs Agassiz, 1836, 184.

Sargodon Plieninger, 1847, 232.

Isch YPtERUS Egerton, 1847, 231.

Fholidotus Williamson, 1849, 244.

Pleurolepis Quenstedt, 1852, 251.

Prionopleurus ${ }^{121}$ Fischer de Waldheim, 1852.

SERRolepis Quenstedt, 1852, 251.

ÆicH MODUS Egerton, 1854, 256.

Dipteronotus Egerton, 1854, 256.

Heterostichus Wagner, 1860, 300.

HOM COLEPIS Wagner, 1860, 300.
Urocomus Costa, 1862, 312.

Scrobodus Von der Marck, 1863, 327.

Hzterostrophus Wagner, 1863, 329.

Plesiodus Wagner, 1863, 329.

Cleithrolepis Egerton, 1864, 330.

Euvlepidotus Egerton, 1868, 349.

Omalopleurus Costa, 1873, 368.

Gyrolepidotus Rohon, 1889, 448.

Archaosemionotus Deecke, 1889, 445.

Pristisomus Woodward, 1890, 453.

DOLICHOPTERUS Compter, 1891, 454.

Lepidopterus Pohlig, 1892, 459.

Prolepidotus Michael, 1893, 462.

ZitheOLEPIS Woodward, 1895, 470.

APH NELEPIS Woodward, 1895, 470.

Paraikichthys Ameghino, 1899, 484.

Eosemionotus ${ }^{122}$ Stolley, 1920.

${ }^{119}$ Written Dapedius by Agassiz.

${ }^{120}$ Afterwards written Lepidotus.

${ }^{121}$ Bull. Sci. Imp. Nat. Moscow, 25: 171; type P. bronni Fischer. (Omitted in "Genera of Fishes.")

${ }^{122}$ Op. cit., 68; orthotype Allolepidotus vogelii Fritsch. 


\section{Order HOLOSTEI \\ Suborder GINGLYMODI \\ (Lepidostei)}

\section{Family 120 . ASPIDORHYNCHID $\mathbb{E}$}

Aspidorhynchus Agassiz, 1833, 177.

Belonostomus Agassiz, 1834, 180.

Dichelospondylus Costa, 1856, 268.

Ophirachis Costa, 1856, 268.
Platycerhynchus Costa, 1864, 330. HEMIRHYNCH US Kner, 1867, 346.

Apateopholis Woodward, 1890, 453. Vinctifer Jordan, 1920, 570.

\section{Family 121. LEPISOSTEID压}

(Gar-Pikes)

Acus Catesby, 1771, 31.

Psalisostomus Klein, 1781, 44.

Lepisosteus ${ }^{123}$ Lacépède, 1803, 66.

Sarchirus Rafinesque, 1818, 109.

Litholepis $^{124}$ Rafinesque, 1818, 109.
Cylindrosteus Rafinesque, 1820, 112.

Atractosteus Rafinesque, 1820, 112.

Pneumatosteus Cope, 1870, 356.

Clastes Cope, 1873, 368.

${ }^{123}$ Usually corrected to Lepidosteus.

${ }^{124}$ Mythical Gar-Pike.

\section{Order HALECOMORPHI}

(Cycloganoidei; Protospondyli; Amioidei)

\section{Family 122. PACHYCORMID㤅}

(Pelecopteridce; Erisichthida; Protosphyranida)

SaUropsis Agassiz, 1832, 140.

PACHyCormus Agassiz, 1833, 177.

SaURostomus Agassiz; 1833, 177.

Protosphyrana Leidy, 1856, 273.

PAChylePIS Quenstedt, 1858, 283.

Lycodus Quenstedt, 1858, 283.

Hypsocormus Wagner, 1860, 300.

Euthynotus Wagner, 1860, 300.

Cyclospondylus Quenstedt, 1867, 347.

Erisichthe Cope, 1872, 364.

Cephenoplosus Sauvage, 1874, 377.
Pseudothrissops Sauvage, 1875, 381.

Heterothrissops Sauvage, 1875, 381.

Pelecopterus Cope, 1875, 379.

Agassizia Vetter, 1881, 418.

Diplolepis Vetter, 1881, 418.

Leedsia Woodward, 1889, 448.

LeEDsichthys Woodward, 1889, 449.

Parathrissors Sauvage, 1891, 456.

Prosauropsis Sauvage, 1893, 462.

Protosauropsis Sauvage, 1894, 465.

\section{Family 123. MACROSEMIID $\approx$}

Notagogus Agassiz, 1833, 177.

Macrosemius Agassiz, 1834, 179.

Propterus Agassiz, 1834, 179.

Orhiopsis Agassiz, 1834, 179.

DisticholePIS Thiollière, 1850, 247.

Petalopteryx Pictet, 1850, 247.

Elenniomeus Costa, 1850, 245.

Rhynchoncodes Costa, 1850, 245.
Calignathus Costa, 1853, 253.

Histionotus Egerton, 1854, 256.

LEGNONOTUS Egerton, 1854, 256.

Orth URUs Kner, 1866, 341.

Aphanepygus Bassani, 1879, 398.

Eusemius Vetter, 1881, 418.

Macrepistius Cope, 1894, 464.

ENCHELYOLEPIS Woodward, 1918, 565. 
Family 124. FURID互 (Eugnathide; Isopholide)

The name Eugnathus is preoccupied. Of the substitute names, Furo and Isopholis, the former has priority.

URAUS Agassiz, 1832, 140.

Ptycholepis Agassiz, 1832, 140.

Caturus Agassiz, 1834, 179.

Eugnathus Agassiz, 1839, 194.

Amblysemius Agassiz, 1844, 217.

Conodus Agassiz, 1844, 217.

Furo Gistel, 1848, 236.

Strobilodus Wagner, 1851, 250.

Lof Hiostomus ${ }^{126}$ Egerton, 1852, 250.

Endactis Egerton, 1858, 280.

Macropoma (Agassiz) Egerton, (1844) $1858,217$.

Callopterus Thiollière, 1858, 284.

Вгаснуіснтну Winkler, $1862,320$.

EURYCORMUS Wagner, 1863, 329.

Ditaxionus Owen, $1866,342$.

ThlatTodus Owen, 1866, 342.
EuryPoma Huxley, 1866, 341.

IsocoluM Egerton, 1868, 349.

Osteorhachis Egerton, 1868, 349.

Heterolepidotus Egerton, 1872, 365.

Harpactes Egerton, 1876, 385.

HARPACTIRA Egerton, 1876, 385.

LissolePIS Davis, 1884, 227.

Isop HoLIS Zittel, 1887, 439.

CRenilepis Dames, 1888, 440.

Neorhombolepis Woodward, 1888, 444.

Allolepinotus Deecke, 1889, 445.

Otomitla Felix, 1891, 454.

Asthenocormus Woodward, 1895, 470.

Sfaniolepis Kramberger, 1905, 514.

Dallopterus Abel, 1906, 516.

Ainia Jordan, 1919, 567.

${ }^{125}$ Misprinted Lophistomus in "Genera of Fishes."

\section{Family 125. ARCH正OM正NID王}

Arch fomenne Woodward, 1895, 470.

\section{Family 126. LIODESMID \&}

LIODEsMus Wagner, 1863, 377, $329 . \quad$ LCPHiURUs Vetter, 1881, 418.

\section{Family 127. AMIIDE (Amiatida)}

(Bow-fins)

Amia Linnæus, 1766, 23.

Amiatus Rafinesque, 1815, 91.

Megalurus Agassiz, 1833, 177.

Cyclurus Agassiz, 1844, 218.

Noteus Agassiz, 1844, 218.

Synergus Gistel, 1848, 236.
Amiopsis Kner, 1863, 327.

Pappichthys Cope, 1873, 368.

Protamia Leidy, $1873,370$.

Hypamia Leidy, 1873, 370.

URoCles Jordan, 1919, 567.

Superorder TELEOSTEI

(The Bony Fishes)

Order ISOSPONDYLI

(Physostomi; Malacopterygii)

(The Soft-rayed Fishes)

Suborder PHOLIDOPHOROIDEI

Family 128. PHOLIDOPHORIDA

Рholidophorus Agassiz, 1832, 140.

Microps Agassiz, 1833, 177.
Ceramurus (Egerton) Brodie, 1845, 226.

Periergus Gistel, 1848, 237. 
Noтнозомus ${ }^{120}$ (Agassiz) Egerton, (1844) 1858, 217, 280.

THORACOPTERUS Bronn, 1858, 279. Pleuropholis Egerton, 1858, 280. Phozidopleurus Bronn, 1858, 279. Megalopterus Kner, 1866, 341.
Peltopleurus Kner, $1866,341$.

Pterygopterus Kner, 1867, 346.

Prohalecites Deecke, 1889, 445.

Baleitcitthys Rohon, 1890, 452.

Gigantopterus Abel, 1906, 516.

${ }^{120}$ Misprinted Nothosoma in "Genera of Fishes." Nothosomus Bassani, 434, "Genera of Fishes," should be canceled.

\section{Family 129. OLIGOPLEURIDA}

Oligopleurus Thiollière, 1850, 247.

IoNoscopus $^{127}$ Costa, 1853, 253.

Holochondrus $^{\text {177. }}$ Thiollière, 1854.

MACRORHIPIS Wagner, 1863, 329.

Enoscopus Costa, 1864, 330.
Opsigonus ${ }^{123}$ (Kramberger). Bassani, $1882,418$.

Spathiurus Davis, 1887, 431.

AmphilaPh URUS Davis, 1887, 431.

ATtAKEOPSIS Thiollière, 1858, 284, 366.

${ }^{127}$ Altered to Enoscopus by authors.

177. Name undefined.

${ }^{128}$ Opsigonus, 1895, "Genera of Fishes," p. 469, should be canceled.

\section{Family 130 . LEPTOLEPID压}

Ichthyolithus Germar, 1826, 120.

Lleptolepis Agassiz, 1832, 141.

Thrissops Agassiz, 1833, 177.

Ascalabos Münster, 1839, 196.

Aithalion Münster, 1842, 212.

Megastoma Costa, 1845, 226.

Oxygonius (Agassiz) Brodie, 1845, 226.

Tharsis Giebel, 1848, 233.

LYCOPTERA. Müller, 1848, 239.
Sarginites Costa, 1850, 245.

Eurystethus Sauvage, 1877, 392.

Ctenolepis (Agassiz) Woodward, (1844) 1895, 217, 470.

VidAlia Sauvage, 1903, 506.

Tharrhias Jordan \& Branner, 1908, 528.

Cearana J. \& B., 1908, 528.

Parathrissops Eastman, 1914, 553.

PACHYTHRISSOPS ${ }^{128}$ Woodward, 1918.

${ }^{128 \pi}$ Monogr. Pal. Soc., 1918, 128, orthotype $P$. lævis, Woodward.

\section{Suborder ELOPOIDEA}

\section{Family 131. ELOPID $\mathbb{E}$}

\section{(Ten-pounders)}

This family, of which but one genus is now living, shows much variation in dentition. Spaniodon, Enneles, and other genera differing widely from the usual type. It should perhaps be further subdivided. With the Megalopida and Pachyrhizodontida, the Elopidae agrees with Amia in the presence of the gular plate, wanting in all other groups of fishes.

Elops Linnæus, 1766, 23.

Mugilomorus Lacépède, 1803, 67.

Trichonotus Rafinesque, 1815, 90.

RHACOLEPIS ${ }^{120}$ Agassiz, 1841, 208.

Calamopleurus Agassiz, 1841, 208.
Aulolepis Geinitz, 1849, 408.

Sa URopsidium Costa, 1850, 245.

SPANiodon Pictet, 1850, 247.

Histialosa Gervais, 1855, 409.

Fi.lopopsis Heckel, 1856, 270.

${ }^{120}$ Misprinted Phacolepis in the original paper. 
RhabDolepis Von Der Marck, 1863, 327. Cexus ${ }^{130}$ Costa, 1864, 264.

Hyptius Costa, 1864, 330.

HolColepis Von Der Marck, 1865, 339, 352.

Dypterolepis (Steinla) Geinitz, 1868, 349.

Micropetalolepis (Steinla) Geinitz, 1868, 349.

Perigram matolepis (Steinla) Geinitz, 1868, 349.

Acrogram matolepis (Steinla) Geinitz, 1868,350 .

Leptogrammatolepis (Steinla) Geinitz, $1868,349$.

A N $\mathbb{A}$ DOPOGON Cope, 1871,360 .

THRISSOPATER Günther, 1872, 365.

THRISSOPTEROIDES Von der Marck, 1873, 370.

${ }^{130}$ Misprinted Cacus.

Family 132. RAPHIOSAURID王 (Pachyrhizodontide)

Megalodon Agassiz, 1835, 181.

Raphiosaurus Owen, 1842, 212.

PACHYRhizodus (Agassiz) Dixon, 1850, 246.

Conosaurus Gibbes, 1850, 246.

ONCHOSAURUS $^{131}$ Gervais, 1852, 408.
Osmerolepis Fritsch, 1875, 379.

Hemielopopsis Bassani, 1879, 398.

I.rwisia Davis, 1885, 431.

Protelops Laube, 1885, 433.

HyPSOSPONDYLUS Kramberger, 1885, 433.

LYROLEPIS Romanovsky, 1886, 436.

Esocelops Woodward, 1901, 495.

E.Lopides (Ag.) Woodward, 1901, 495, 219.

Notelops Woodward, 1901, 495.

Eurygnathus (Agassiz) Woodward, (1845) $1901,224$.

Parelops Fritsch \& Bayer, 1902, 498.

Dinelops Woodward, 1907, 527.

En Neles Jordan \& Branner, 1908, 528.

Helmint holepis Cockerell, 1919, 565.

Ectasis Jordan \& Gilbert, 1919, 569.

ENNELICHTHYs Jordan, 1920, 570.

BRANNERION Jordan, 1920, 570.

${ }^{131}$ It is likely that both Onchosaurus and Ischyrhiza are reptilian teeth.

\section{Family 133. MEGALOPID $\mathbb{E}$}

(Tarpons; Grande-Écailles)

Amia Browne, 1789, 46.

Megalops Lacépède, 1803, 66.

Oculeus (Commerson) Lacépède, 1803, 70.

\section{Family 134. GANOLYTID王}

Etringus Jordan, 1907, 525.

Ganolytes Jordan, 1919, 568.

GANOËssus Jordan, 1920, 571.

\section{Suborder ALBULOIDEI}

Family 135. ALBULIDA

\section{(Lady-fishes)}

Albula Gronow, 1763, 20.

Albula Scopoli, 1777, 42.

Albula Bloch \& Schneider, 1801, 59.

Butyrinus Lacépède, 1803, 65.

Glossodus Cuvier, 1815, 93.

Pisodus Agassiz, 1845, 227.
Conorhynchus (Nozemann) Gill, 1861, 303.

Petalolepis (Steinla) Geinitz, 1868, 350.

Apsopelix Cope, 1871, 360.

Cycloides $^{182}$ Winkler, 1878, 398.

Dixonina Fowler, 1911, 538.

${ }^{132}$ Very doubtful, perhaps not a fish scale. 
Family 136. THRYPTODONTIDE (Plethodontide) $)^{133}$

Plethodus Dixon, 1850, 245.

Anogmius Cope, 1877, 388.

Thryptodus Loomis, 1900, 490.

Pseudothiryptodus Loomis, 1900, 490.

${ }^{133}$ The name Plethodontida is already used for a family of Salamanders.

\section{Suborder CLUPEOIDEI}

\section{Family 137. CHANID瓜}

Chanos Lacépède, 1803, 67.

Lutodeira Rüppell, 1828, 122, 184.

Scoliostomus Rüppell, 1828, 122.
Ptycholepis Gray, 1842, 211.

Prochanos Bassani, 1879, 398.

Chanoides Woodward, 1901, 495.

\section{Family 138. ANCYLOSTYLID E}

A single genus, apparently related to the Chanida.

Ancylostylos Kramberger, 1895, 469.

\section{Family 139. CHIROCENTRID $A$}

It is not certain that all of the genera named below belong to one family, or to the same family as the single living genus, Chirocentrus.

Monopteros Volta, 1796, 169.

Chirocentrus Cuvier, 1817, 99.

Saurocephalus Harlan, 1824, 118.

Saurodon Hays, 1830, 136.

Platinx Agassiz, 1835, 181.

Cologaster Agassiz, 1835, 181.

Thrissopterus Heckel, 1856, 270.

Andreiopleura Costa, 1865, 336.

Heteroleiris Costa, 1865, 336.

TELEPHOLIS Marck \& Schlüter, 1865, 339.

Credem nolepis Steinla, 1868, 349.
Polypterolepis Steinla, 1868, 350.

Oölepis Steinla, 1868, 350.

Agram matolepis Steinla, 1868, 350.

Crom m iolepis Steinla, 1868, 350.

Cyclolepis Geinitz, 1868, 349.

Hemicyclus Geinitz, 1868, 350.

Ptycholepis Steinla, 1868, 350.

Heliolepis Steinla, 1868, 350.

Mixogrammatolepis Steinla, 1868, 350.

Chiromystus Cope, 1886, 434.

Eubiodectes Hay, 1903, 503.

\section{Family 140. ERYTHRINOLEPID E}

ERYthrinolepis Cockerell, 1919, 566.

\section{Family 141. PTEROTHRISSIDE (Bathythrisida)}

Kymatopetalepis (Steinla) Geinitz, $1868,349$.

Pterothrissus Hilgendorf, 1877, 390.
Bathythrissa Günther, 1877, 389.

Syntegmodus Loomis, 1900, 490.

Chicolepis Cockerell, 1919, 565.

\section{Family 142. ICHTHYODECTID $\&$}

Hypsodon Agassiz, 1887, 186, 219.

Chirocentrites Heckel, 1849, 242.

Tomognathus Dixon, 1850, 246.

Spathodactylus Pictet \& Loriol, 1859, 292.

Andreiopleura Costa, 1865, 336.
Ichth yodectes Cope, 1870, 356.

Xiphiactinus Leidy, 1870, 358.

Prymnetes Cope, $1871,360$.

Portheus Cope, 1872, 364.

Gillicus Hay, 1898, 480.

Proportheus Jackel, 1909, 533. 


\title{
Family 143. SAURODONTIDEE
}

Saurocephalus Harlan, 1824, 118.

Daptinus Cope, $1872,364$.

SAURODON Hays, 1830, 136.

\section{Family 144. GONORHYNCHID}

It is doubtful whether the extinct forms of this group (Notogoneus, Charitostomus) really belong to the same family as the living Gonorhynchus.

Gonorhynchus Gronow, 1763, 19.

Gonorhynchus Scopoli, 1777, 42.

Rhynchana Richardson, 1844, 407.

\section{Family 145. NOTOGONEID瓜}

ANormurus Blainville, 1818, 171.

Sphenolepis Agassiz, 1844, 218.

Solenognathus Pictet, $1866,342$.

Сharitosomus Marck, 1885, 433.
Notogoneus ${ }^{184}$ Cope, 1885, 430.

Protocatostomus Whitfield, 1890, 453.

GONORH YNCHOPS Cockerell, 1919, 566.

\begin{abstract}
${ }^{184}$ Cockerell observes, in lit., May 13, 1922: "As to Notogoneus, I don't think it could be a characin. If not a gonorhynchid, then the type of a new family, Notogoneide. I suppose the Gonorhynchid type of scales, originating during the Mesozoic, may have persisted in several distinct branches of the original stem, of which our modern Gonorhynchida constitute one only. One of the other branches would be represented by Notogoneus. I think the resemblance is too close for convergence from entirely different stems."

The view expressed above implies that the Cretaceous Gonorhynchops and Charitosomus are probably not true Gonorhynchida, in the modern sense, but represent a family ancestral to the modern one. Whether such a fanily could be defined is another matter.
\end{abstract}

\section{Family 146. HIODONTID丑}

\section{(Moon-fishes)}

Hiodon $^{\text {185 }}$ Le Sueur, 1818, 107.

Glossodon Rafinesque, 1818, 108.

Amphiodon Rafinesque, 1819, 110.
Clodalus Rafinesque, 1820, 111.

Glossodon Heckel, 1842, 211.

Elattonistius Gill \& Jordan, 1877, 390.

${ }^{285}$ Later more correctly written Hyodon.

\section{Family 147. CLUPEID平}

\section{(Herrings)}

Clupea Linnæus, 1758, 15.

Harengus Geoffroy, 1767, 24.

Harengus Catesby, 1771, 31.

Harengus Klein, 1775, 38.

Alosa Linck, 1790, 49.

Odontognathus Lacépède, $1800,56$.

Pomatias Bloch \& Schneider, 1801, 60.

Gnathobolus B. \& S., 1801, 60.

Opisotomus (Commerson) Lacépède, $1803,70$.
Trichis (Plumier) Lacépède, 1803, 73.

Pristigaster Cuvier, 1817, 99.

Pomolobus Rafinesque, 1820, 111.

Alosa $^{188}$ Cuvier, 829, 130.

Corica Hamilton, 1822, 115.

Raconda Gray, 1831, 138.

Apterygia Gray, 1833, 179.

Apterogașterus De La Pylaie, 1835, 184.

Platygaster Swainson, 1839, 203.

Ilisha Gray, 1845, 227.

\footnotetext{
${ }^{230}$ Also written Alausa.
} 
Spratella Cuvier \& Valenciennes, 1847, Potamalosa Ogilby, 1896, 475. 231.

Kowala Cuv. \& Val., 1847, 231.

Clupeonia Cuv. \& Val., 1847, 231.

Omochetus Ogilby, 1897, 477.

Odaxothrissa Boulenger, 1899, 485.

Leptichtics Stewart, 1899, 487.

Rogenia Cuv. \& Val., 1847, 231.

Sardinella Cuv. \& Val., 1847, 231.

Harengula Cuv. \& Val., 1847, 231.

Histiothrissa Woodward, 1901; 495.

Halecopsis (Agassiz) Woodward, (1844) 1901, 217, 218, 496.

Alausa Cuv. \& Val., 1847, 231.

Meletta Cuv. \& Val., 1847, 231.

Pellona Cuv. \& Val., 1847, 231.

Amblygaster Bleeker, 1849, 240.

Histiurus Costa, 1850, 245.

Clupeoides Bleeker, 1851, 247.

Dascillus Gronow, 1854, 259.

Clupeichthys Bleeker, 1855, 263.

Sardinia Poey, 1860, 299.

Alosina Wagner, 1860, 300.

Opisthopterus Gill, 1861, 303.

Alausella Gill, is61, 303.

Opisthonema Gill, 1861, 303.

Brevoortia Gill, 1861, 303.

SCOMBRociupea Kner, 1863, 327.

UROPTERINA LiOY, 1865, 338.

Ptericepralina Lioy, 1865, 339.

PSEUdoberyx Pictet \& Humbert, 1866, 342.

Pellonula Günther, 1868, 351.

Chirocentrodon Günther, 1868, 351.

Paralosa Bleeker, 1872, 362.

Syllemus Cope, 1875, 379.

Clupeonella Kessler, 1877, 391.

Diplomystus Cope, 1877, 388.

Clupeors Sauvage, 1880, 402.

HaceUeTia ${ }^{137}$ Szajnocha, 1886, 436.

Hyperlophus Ogilby, 1892, 459.

Lile Jordan \& Evermann, 1896, 473.

Microthrissa Boulenger, 1902, 496.

Lyroleris Romanovskii, 1904, 510.

Copeichthys Dollo, 1904, 507.

Sardina Antipa, 1905, 512.

Knightia Jordan, 1907, 525.

Ellipes Jordan, 1910, 536.

Dastilbe Jordan, 1910, 536.

Heringia Fowler, 1911, 539.

Gudusia Fowler, 1911, 539.

Rhinosardinia Eigenmann, 1912, 543.

Clupeonella Berg, 1913, 546.

Ellimma Jordan, 1913, 548.

Zunasia Jordan \& Metz, 1913, 548.

Caspialosa Berg, 1915, 555.

Paralosa Regan, 1916, 560.

Ethmalosa Regan, 1916, 560.

Hilsa Regan, 1916, 560.

Ethmidium Thompson, 1916, 561.

Pœcilothrissa Regan, 1917, 563.

Potamothrissa Regan, 1917, 563.

Cynothrissa Regan, 1917, 563.

Stolothrissa Regan, 1917, 563.

Limnothrissa Regan, 1917, 563.

IQvius Jordan, 1919, 568.

Xyne Jordan \& Gilbert, 1919, 568.

Elim michrнy Jordan, 1919, 568.

Alisea J. \& G., 1919, 568.

Xyrinius J. \& G., 1919, 569.

Quisque Jordan, 1920, 571.

${ }^{137}$ Not identifiable.

\section{Family 148. DUSSUMIERIID}

\section{(Round Herrings)}

Stolephorus ${ }^{138}$ Lacépède, 1803, 67.

Dussumieria Cuvier \& Valenciennes, $1847,231$.

Spratelloides Bleeker, 1851, 248.

Etrumeus Bleeker, 1853, 253.

Leptogaster Bleeker, 1872, 362.
Perkinsia Eigenmann, Rosa S., 1891, 454.

Jenkinsia Jordan \& Evermann, 1896, 47.3.

Rhomurus Jordan, 1919, 568.

Smithites Jordan \& Gilbert, 1919, 568.

Qu

Lygisma J. \& G., 1919, 568.

${ }^{188}$ This application of this name is still uncertain. It originally included a species of Dussumieriida and one of Engraulida. 


\section{Family 149. DOROSOMIDEE \\ (Gizzard Shads)}

Clupanodon $^{138}$ Lacépède, 1803, 68.

Thrissa Rafinesque, 1815, 90.

Dorosoma Rafinesque, 1820, 111.

Gosostoma Van Hasselt, 1823, 172.

Chatoëssus Cuvier, 1829, 130.

Anodontostoma Bleeker, 1849, 241.
Signalosa Evermann \& Kendall, 1898, 480.

Konosirus Jordan \& Snyder, 1900, 490.

Nematalosa Regan, 1916, 560.

Gonialosa Regan, 1916, 560.

${ }^{239}$ The proper application of this name has been questioned. Apparently it should replace Konosirus among the Dorosomida.

\section{Family 150. ENGRAULIDE}

\section{(Anchovies)}

Menidia Browne, 1789, 46.

Stolephorus ${ }^{140}$ Lacépède, 1803, 67.

Encrasicholus (Commerson) Lacépède, $1803,71$.

Mystus Lacépède, 1803, 67.

Engraulis Cuvier, 1817, 98.

Thrissa ${ }^{141}$ Cuvier, 1817, 98.

Encrasicholus Fleming, 1828, 122.

Coilia Gray, 1831, 138.

Trichosoma Swainson, 1839, 203.

Setipinna Swainson, 1839, 203.

Chætomus McClelland, 1844, 220.

Leptomurus Bleeker, 1849, 241.
Stethochætus Gronow, 1854, 259.

Osteoglossum Basilewski, 1855, 262.

Telara Günther, 1868, 351.

Cetengraulis Günther, 1868, 351.

Pterengraulis Günther, 1868, 351.

Heterothrissa Günther, 1868, 351.

Lycengraulis Günther, 1868, 351.

Lycothrissa Günther, 1868, 351.

Anchovia Jordan \& Evermann, 1895, 468, 473.

Anchoviella Fowler, 1911, 539.

Thrissocles Jordan, 1917, 562.

${ }^{x * 0}$ This genus is based on two species, belonging to different families. The final disposition of the name awaits decision.

${ }^{14}$ Later written Thryssa by Cuvier.

\section{Family 151. ALEPOCEPHALID $\approx$}

Triurus Lacépède, 1800, 56.

Pomatias Bloch \& Schneider, 1801, 60.

Alepocephalus Risso, 1820, 113.

Esunculus Kaup, 1856, 272.

Xenodermichthys Günther, 1878, 395.

Bathytroctes Günther, 1878, 395.

Platytroctes Günther, 1878, 395.

Aleposomus ${ }^{142}$ Gill, 1884.

Leptoderma Vaillant, 1886, 436, 444.

Anomalopterus Vaillant, 1886, 436, 444.

Aulostomatomorpha Alcock, 1890, 449.

Tauredophidium Alcock, 1890, 449.
Narcetes Alcock, 1890, 449.

Conocara Goode \& Bean, 1895, 467.

Talismania G. \& B., 1895, 467.

Mitchillina Jordan \& Evermann, 1896, 473.

Ericara Gill \& Townsend, 1897, 477.

Leptochilichthys ${ }^{13}$ Garman, 1899.

Dolichopteryx Brauer, 1901, 492.

Asquamiceps Zugmayer, 1911, 541.

Xenognathus Gilbert, 1915, 556.

Benthosphyraena Cockerell, 1919, 566.

Rouleina ${ }^{144}$ Jordan, 1923.

${ }^{142}$ Amer. Nat., 18: 433; type Aleposomus copei Gill; regarded as a synonym of Xenodermichthys. (Omitted in "Genera of Fishes.")

143 "Deep Sea Fish," 284 ; orthotype L. agassizii. (Omitted in "Genera of Fishes.")

14 New genus; type Aleposomus gïntheri Alcock, same as Aleposomus Roule, not of Gill, which is a synonym of Xenodermichthys Günther. 
Family 152. MACRISTIID \&

Macristium Regan, 1903, 505.

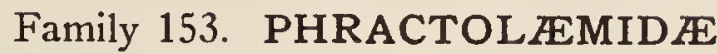

Phractolæmus ${ }^{145}$ Boulenger, 1901.

${ }^{145}$ Boulenger, Proc. Zool. Soc. Lond., 3, 1901; type P. ansorgi Boulenger. (Omitted in "Genera of Fishes.")

\section{Family 154. KNERIID F}

Kneria Steindachner, 1866, 343.

Xenopomatichthys Boulenger, 1910, 534.

\section{Family 155. CROMERIIDE}

Cromeria Boulenger, 1901, 492.

\section{Suborder OSTEOGLOSSOIDEA}

\section{Family 156 . OSTEOGLOSSID $\mathbb{E}$}

This family and the next two should perhaps be merged into one.

Osteoglossum (Vandelli) Cuvier, 1829, Dapedoglossus Cope, 1877, 388. 131.

Ischnosoma Spix, 1829, 132.

Cladocyclus s' Agassiz, 1841, 208.

Platops Owen, 1901, 260.

Brychetus (Agassiz) Woodward, 1901, 224, 495.

Phareodus ${ }^{147}$ Leidy $1873,370$.

Pomaphractus (Agassiz) Woodward, (1845) 1901, 224.

14 The original species of this genus from Brazil belongs certainly to or near the Osteoglossid $a$, and is distinguished by the great development of intermuscular bones, attached to the occiput forming a brush three times as long as the head and extending backward along the sides, looking in the fossil example like the wing of a flying fish. Cockerell suggests that it may be an ancestral characin.

${ }^{147}$ This name seems to me tenable; the description of the genus is included in that of the species, as with Protamia and Hypamia of the same paper.

\section{Family 157. ARAPAIMID王}

Sudis Cuvier, 1817, 99.

Vastres Cuvier \& Valenciennes, 1846, 228.

Arapaima Müller, 1843, 216, 229.

\section{Family 158. CLUPISUDIDA (Heterotida)}

Clupisudis Swainson, 1839, 202.

Helicobranchus Hyrtl, 1854, 260.

Heterotis (Ehrenberg) M üller, 1843,

$131,216,229$.

\section{Family 159. PANTODONTIDFE}

Pantodon Peters, 1877, 391.

\section{Suborder NOTOPTEROIDEI \\ Family 160. NOTOPTERID $\mathbb{E}$}

Notopterus Lacépède, 1800, 56.

Xenomystus Günther, 1868, 351.

Glanis Gronow, 1854, 259. 


\section{Suborder SCYPHOPHORI}

Family 161. MORMYRIDIE

Mormyrus Linnæus, 1758, 15.

Scrophicephalus Swainson, 1839, 198.

Mormyrops Müller, 1843, 216.

Petrocephalus Marcusen, 1854, 334, 260.

Phagrus Marcusen, 1854, 334.

Marcusenius Gill, 1862, 314.

Mormyrodes Gill, 1862, 314.

Hyperopisus Gill, 1862, 314.

Isichthys Gill, 1862, 317.
Gnathonemus Gill, 1862, 317.

Solenomormyrus Bleeker, 1874, 372.

Campylomormyrus Bleeker, 1874,372 .

Oxxymormyrus Bleeker, 1874, 372.

Stomatorhinus Boulenger, 1898, 478.

Myomyrus Boulenger, 1898, 479.

Genyomyrus Boulenger, 1898, 479.

Hippopotamyrus Pappenheim, 1906, 520.

\section{Family 162. GYMNARCHID王}

Gymnarchus Cuvier, 1829, 131.

\section{Suborder SALMONOIDEI \\ Family 163. SALMONIDZE (Salmon; Trout)}

The next three families are usually and perhaps properly united with Salmonide.

Salmo Linnæus, 1758, 14.

Trutte $^{148}$ Linnaus, 1758, 14.

Trutta Geoffroy, 1767, 24.

Trutta Klein, 1775, 37.

Salvelinitis Nilsson, 1832, 140.

Salvelinus Richardson, 1836, 186.

Baione Dekay, 1842, 210.

Thaumaturus Reuss, 1844, 222.

Fario Cuvier \& Valenciennes, 1848, 233.
Salar Cuv. \& Val., 1848, 233.

Umbla Rapp, 1854, 261.

Oncorhynchus Suckley, 1860, 299.

Hypsifario Gill, 1862, 317.

Hucho Günther, 1866, 340 .

Rhabdofario Cope, 1870, 356.

Cristivomer Gill \& Jordan, 1878, 395.

Epitomynis Schulze, 1889, 448.

Phyllogephyra Boulenger, 1898, 479.

${ }^{148}$ This name with Osmeri, Coregoni, and Characini were given in plural form only by Linnæus.

${ }^{149}$ Given originally in plura! form only, latinized by Richardson, 1836.

\section{Family 164. PLECOGLOSSID}

(Ayu)

Plecoglossus Temminck \& Schlegel, 1846, 230.

\section{Family 165. COREGONID $\#$}

(White-fishes; Ciscoes)

Coregoni ${ }^{250}$ Linnæus, $1758,14$.

Tripteronotus Lacépède, 1803, 65.

Coregonus Lac., 1803, 66.

Stenodus Richardson, 1836, 185.

Argyrosomus Agassiz, 1850, 245.

Brachymystax Günther, 1866, 340.

Luciotrutta Günther, 1866, 340.
Leucichthys Dybowski, 1874, 375.

Allosomus ${ }^{151}$ Jordan, 1878.

Prosopium Milner, 1878, 395.

Cisco Jordan \& Evermann, 1911, 539.

Thrissominus Gill, 1911, 539.

Irillion Jordan, 1919, 567.

LEUCiCHTH Yops Cockerell, 1919, 566.

${ }^{150}$ Given in plural form only, as Coregoni.

${ }^{161}$ Man. Vert., Ed. 2, 361; type Coregonus tullibee Richardson. (Omitted in "Genera of Fishes.") 


\section{Family 166. THYMALLID E}

(Grayling)

Thymallus Linck, 1790, 49. Thymallus Cuvier, 1829, 130. Choregon Minding, 1832, 140. Orthocolus Gistel, 1848, 237.
Cyclolepis Geinitz, 1868, 349.

Prototh y mallus Laube, 1901, 495.

Thymalloides Berg, 1908, 527.

Salmothymus Berg, 1908, 527.

\section{Family 167. OSMERID $Æ$}

(Smelt)

A group scarcely distinct as a family from the Argentinida.

Osmeri $^{\text {i52 }}$ Linnæus, 1758, 14.

Osmerus Lacépède, 1803, 66.

Mallotus Cuvier, 1829, 130.

Eperlanus Gaimard, 1849, 249.

Eperlanus Basilewski, 1855, 262.

Thaleichthys Girard, 1859, 290.
Mesopus ${ }^{153}$ Gill, 1862, 313.

Hypomesus Gill, 1862, 313.

Spirinchus Jordan \& Evermann, 1896, 473.

Therobromus Lucas, 1898, 482.

Eperlanio Jordan, 1919, 567.

${ }^{163}$ In plural form only, as Osmeri.

${ }^{163}$ A name left in type through failure to correct proof sheets.

\section{Family 168. ARGENTINID $\$$}

Argentina Linnæus, 1758, 15.

Silus Reinhardt, 1833, 179.

Acantholepis Kröyer, 1846, 229.
Glossanodon Guichenot, 1866, 341.

Leuroglossus Gilbert, 1890, 451.

\section{Family 169. MICROSTOMID \&E}

Microstoma Cuvier, 1817, 99.

Halaphya Günther, 1889, 447.

Nansenia Jordan \& Evermann, 1896, 473.

\section{Family 170. BATHYLAGID $\mathbb{E}$}

Bathylagus Günther, 1878, 395.

Bathymacrops Gilchrist, 1922. (See Index.)

\section{Family 171. RETROPINNIDÆ \\ (New Zealand White-bait)}

Retropinna Gill, 1862, 313.

Richardsonia Steindachner, 1866, 343.

\section{Family 172. SALANGIDÆ}

(Chinese White-bait)

Albula Osbeck, 1762, 17.

Salanx Cuvier, 1817, 99.

Leucosoma Gray, 1831, 139.
Salangichthys Bleeker, 1860, 293.

Parasalanx Regan, 1908, 530.

Hemisalanx Regan, 1908, 530.
Galaxias Cuvier, 1817, 99.

\section{Family 173. GALAXIID}

Mesites Jenyns, 1842, 212.
Neochanna Günther, 1867, 345.

Austrocobitis Ogilby, 1899, 487.

Family 174. APLOCHITONID瓜

Aplochiton $^{154}$ Jenyns, 1842, 212.

Farionella Cuvier \& Valenciennes, 1849, 242.
Prototroctes Günther, 1864, 333.

Jenynsella Ogilby, 1908, 529.

Lovettia McCulloch, 1915, 556.

${ }^{154}$ Later spelled Haplochiton. 


\section{Suborder ENCHODONTOIDEI \\ Family 175. ENCHODONTID死}

Some of the genera named below have been referred, perhaps justly, to the Dercetida, a family which may be indeed closely related to the Enchodontider.

Halec Agassiz, 1834, 180.

Enchodus Agassiz, 1835, 181.

Isodus Heckel, 1849, 243.

SAURORHAMPH US Heckel, 1850, 246.

Pomognathus Dixon, 1850, 245.

Eurypholis Pictet, 1850, 247.

Phasganodus Leidy, 1858, 283.

ISCHYROCEPHALUS von der Marck, 1858, 283.

Pal folycus von der Marck, 1863, 327.

Plinthophorus Günther, 1864, 333.
Archagogadus Marck \& Schlüter, 1865, 339.

Tetheodus Cope, 1874, 375.

Trichiurides Winkler, 1878, 398.

Solenodon Kramberger, 1881, 416.

HoLCoDon Kramberger, 1885, 433.

Phylactocephalus Davis, 1885, 431.

Pantopholis Davis, 1885, 431.

Eurygnathus Davis, 1885, 431.

DipNolepis Fritsch, 1893, 461.

Halecodon Cockerell, 1919, 566.

\section{Suborder STOMIAT IOIDEI}

We may apply this term to a group of isospondylous fishes, which for the most part retain the adipose fin and which have the side of the upper jaw formed by the maxillaries, differing in this regard strongly from the Iniomi, with which they have been usually associated. Most members of both groups are bathypelagic fishes, degenerate in many regards. The division into families as here accepted must be regarded as provisional.

\section{Family 176. STOMIATID正}

Stomias Cuvier, 1817, 99.

Echiostoma Lowe, 1843, 215.

Stomianodon Bleeker, 1849, 241.

Stomiasunculus Kaup, 1860, 297.

Lucifer Döderlein, 1882, 418.

Bathyophilus Giglioli, 1883, 423.

Hyperchoristus Gill, 1883, 424.

Eustomias Filhol, 1884, 427.

Pachystomias Günther, 1887, 438.

Opostomias Günther, 1887, 438.

Photonectes Günther, 1887, 438.

Eustomias Vaillant, 1888, 444.

Photostomias Collett, 1889, 444.

Thaumastomias Alcock, 1890, 449.
Grammatostomias ${ }^{155}$ Goode \& Bean, 1895, 468.

Dactylostomias Garman, 1899, 486.

Macrostomias Brauer, 1902, 497.

Melanostomias Brauer, 1902, 497.

Leptostomias Gilbert, 1905, 513.

Neostomias Gilchrist, 1906, 518.

Trichostomias Zugmayer, 1911, 541.

Nematostomias Zugmayer, 1911, 541.

Benthalbella Zugmayer, 1911, 541.

Lamprotoxus Holt \& Bryne, 1913, 548.

Aristostomias Zugmayer, 1913, 552.

Gnathostomias Pappenheim, 1914, 554.

Zastomias Gilbert, 1915, 556.

${ }^{165}$ Grammatostomias Holt \& Byrne, p. 536, should be canceled. G. flagellibarba Holt \& Byrne is the type of Lamprotoxus, p. 548.

\section{Family 177. TOMOGNATHID $\mathbb{E}$}

Tomognathus Dixon, 1850, 246. 


\section{Family 178. ASTRONESTHID丑}

Astronesthes Richardson, 1844, 222. Bathylychnus Brauer, 1902, 497.

Phænodon Lowe, 1850, 247.

Borostomias Regan, 1908, 529.

\section{Family 179. STYLOPHTHALMID王}

Stylophthalmus Brauer, 1906, 497, 517. Stylophthalmoides Sanzo, 1922. (See Index.)

\section{Family 180. CHAULIODONTID丑}

Vipera Catesby, 1771, 32.

Leptodes Swainson, 1839, 203.

Chauliodus Bloch \& Schneider, 1801, 59.

\section{Family 181. GONOSTOMID死}

Gonostoma Rafinesque, 1810, 83.

Phosichthys ${ }^{\text {1se }}$ Hutton \& Hector, 1872, 366.

Cyclothone Goode \& Bean, 1882, 419.

Sigmops Gill, 1883, 424.

Neostoma ${ }^{186 x}$ Filhol, 1884, 427, 444.
Yarrella G. \& B., 1895, 467.

Bonapartia G. \& B., 1895, 467.

Manducus G. \& B., 1895, 468.

Zaphotias G. \& B., 1898, 482.

Triplophos Brauer, 1902, 497.

AzALoIs Jordan \& Gilbert, 1919, 568.

${ }^{158}$ Later written Photichthys.

${ }^{150 a}$ Neostoma Vaillant, p. 444 , should be canceled.

\section{Family 182. IDIACANTHID瓜}

Idiacanthus Peters, 1876, 386.

Bathyophis Günther, 1878, 394.

\section{Family 183. MAUROLICID王}

Maurolicus Cocco, 1838, 191.

Ichthyococcus Bonaparte, 1840, 141, 206

Coccia Günther, 1864, 333.

Diplophos Günther, 1873, 369.

Vinciguerria Jordan \& Evermann, 1895, 468.

\section{Family 184. OPISTHOPROCTID正}

Opisthoproctus Vaillant, 1888, 443.

Winteria Brauer, 1901, 492.

\section{Family 185 . STERNOPTYCHID压}

Sternoptix ${ }^{187}$ Hermann, 1781, 44.

Argyropelecus Cocco, 1829, 133.

Pleurothyris ${ }^{188}$ Lowe, 1843, 215.
Valenciennellus J. \& E., 1895, 468.

Zalarges Jordan \& Williams, 1895, 469.

Argyripnus Gilbert \& Cramer, 1897, 477.

Lychnopoles Garman, 1899, 486.

Triareus Waite, 1910, 537.

${ }^{157}$ Usually written Sternoptyx.

${ }^{168}$ Misprinted Pleurothysis by Günther.

${ }^{158 a}$ P. Jespersen and A. V. Täning. Nat. Medd., 70:222; orthotype M. obtusirostris

J. \& T.: Cadiz Bay.

\section{Family 186. ANOTOPTERIDE}

Anotopterus Zugmayer, 1911, 541. 


\section{Order LYOPOMI}

\section{Family 187. HALOSAURID王}

Echidnocephalus von der Marck, 1858, Halosaurichthys Alcock, 1889, 444.

283.

Halosaurus Johnson, 1863, 327.

Aldrovandia Goode \& Bean, 1895, 468.

ENCHELURus von der Marck, 1863, 328.

Halosauropsis Collett, 1896, 471.

\section{Family 188. LIPOGENYID压}

Lipogenys Goode \& Bean, 1894, 465.

\section{Order HETEROMI}

\section{Family 189. PRONOTACANTHID Æ}

Pronotacanthus Woodwąrd, 1900, 491.

\section{Family 190. NOTACANTHID.玉}

(Spiny-eels)

Notacanthus Bloch, 1787, 44.

Campylodon Fabricius, 1793, 53.

Acanthonotus Bloch, 1797, 53.

Polyacanthonotus Bleeker, 1874, 372.
Paradoxichthys Giglioli, 1882, 419.

Teratichthys Giglioli, 1882, 419.

Gigliolia Goode \& Bean, 1894, 465.

Macdonaldia G. \& B., 1894, 465.

\section{Suborder HOPLOPLEURI}

\section{(Dercetiformes)}

The relations of this extinct group are still obscure. Some have regarded them as allies of the Enchodonts among the Isospondyli. Others have placed them near the sticklebacks (Gasterosteida). Cockerell, who has the latest guess, places them with the Heteromi.

\section{Family 191. DERCETID压}

Dercetis Agassiz, 1834, 180.

Prionolepis Egerton, 1850, 245.

Palimphemus Heckel, 1862, 319.

Pelargorhynchus von der Marck, 1858 , 283.

Leptotrachelus von der Marck, 1863, 328.
Aspidopleurus Picket \& Humbert, 1866, 342.

Trimnaspis Cope, 1878, 394.

LEPTECODON Williston, 1899, 488.

Apateopholis Woodward, 1890, 453.

\section{Family 192. STRATODONTID $\#$}

Cimolichthys Leidy, 1856, $273 . \quad$ Empo Cope, 1872, 364.

Stratodus Cope, 1872, 364.

\section{Family 193. RHINELLID $\$$}

Rhinellus Agassiz, 1844, $217 . \quad$ IChthyotringa Cope, 1878, 394.

\section{Order SYMBRANCHIA}

Eel-like fishes of tropical rivers and swamps, having distinct maxillary and premaxillary, the latter forming the border of the jaw; apparently not related to the true eels. 


\section{Suborder ICHTHYOCEPHALI}

Family 194. FLUTIDE (Monopteride)

Les Monoptères Lacépède, $1800,56$.

Fluta Bloch \& Schneider, 1801, 60.

Monopterus (Lacépède) Duméril 1806, 56.
Ophicardia McClelland, 1844, 220.

Apterigia Basilewski, 1855, 262.

\section{Suborder HOLOSTOMI}

\section{Family 195. SYNBRANCHID $\approx$}

Synbranchus ${ }^{159}$ Bloch, 1795, 53.

Typhlobranchus Bloch \& Schneiider, 1801, 60.

Unibranchapertura Lacépède, 1803, 68.
Ophisternon McClelland, 1844, 220.

Tetrabranchus Bleeker, 1851, 248.

Unipertura Kaup, 1856, 268.

Macrotrema Regan, 1912, 545.

${ }^{160}$ Usually corrected to Symbranchus.

\section{Family 196. AMPHIPNOID $\$$}

Amphipnous Müller, 1839, 196.

Pneumabranchus McClelland, 1844, 220.

Ophichthys Swainson, 1839, 205.

\section{Suborder ALABIFORMES}

\section{Family 197. ALABID $\mathbb{E}$}

Alabes (Cuvier) Oken, 1817, 101. Cheilobranchus Richardson, 1844, 223.

\section{Order OPISTHOMI}

Eel-shaped fishes, with dorsal spines and with the shoulder girdle attached behind the occiput as in the Heteromi.

\section{Family 198. CHAUDHURIID压}

Chaudhuria Annandale, 1918, 563.

\section{Family 199. MASTACEMBELID $\mathbb{E}$}

Mastacembelus Gronow, 1763, 21.

Mastacembelus Scopoli, 1777, 42.

Macrognathus Lacépède, 1800, 56.
Rhynchobdella Bloch \& Schneider, 1801, 59.

Pararhychobdella Bleeker, 1874, 372.

\section{Order APODES}

\section{Suborder CARENCHELI}

As these eel-shaped fishes have a distinct maxillary and premaxillary, it is very doubtful whether their place is among or even near the eels.

\section{Family 200. DERICHTHYIDEE}

Derichthys Gill, 1884, 428.

\section{Suborder ARCHENCHELI}

Eel-like forms with well developed ventral fins. It is doubtful whether these fishes are really to be placed among the eels. 
Family 201. ANGUILLAVID $\mathbb{E}$

Anguillavus Hay, 1903, 503.

\section{Suborder ENCHEL YCEPHALI \\ Series ANGUILLOIDEI}

Body with rudimentary scales; eggs minute.

\section{Family 202. ANGUILLIDE}

(True Eels)

Anguilla Shaw, 1803, 73.

Tribranchus Peters, 1844, 407.

\section{Fahily 203. SIMENCHELYID死}

Simenchelys ${ }^{100}$ (Gill) Goode \& Bean, Conchognathus Collett, 1889, 444. 1879.

Gymnosimenchelys Tanaka, 1908, 531.

${ }^{100}$ Bull. Essex Inst., 27; orthotype Simenchelys parasilicus Gill. (Omitted in "Genera of Fishes.")

Family 204. SYNAPHOBRANCHID $\approx$

Synaphobranchus Johnson, 1862, 319. Nettophichthys Holt, 1891, 455.

Histiobranchus Gill, 1883, 424.

\section{Family 205. ILYOPHID死}

Ilyophis Gilbert, 1891, 454.

\section{Series CONGROIDEI}

Body scaleless; eggs large.

Family 206. DYSOMMID压

Dysomma Alcock, 1889, 444.

Dysommopsis Alcock, 1891, 454.

Family 207. XENOCONGRID $\mathbb{E}$

Xenoconger Regan, 1912, 545.

\section{Family 208. MYROCONGRID丑}

Myroconger Günther, 1870, 357.

Family 209. MURÆNESOCIDÆ

Murzesox McClelland, 1844, $220 . \quad$ Brachyconger Bleeker, 1865, 335.

Cynoponticus Costa, 1846, 228. Congresox Gill, 1890, 451.

Family 210. SAUROMURANESOCID五

Sauromurænesox Alcock, 1889, 444.

Family 211. CONGRID在161 (Leptocephalida)

(Conger Eels)

Leptocephalus Gronow, 1763, 22.

Conger (Cuvier) Oken, 1817, 101.

Conger Klein, 1775, 37.

Ariosoma Swainson, 1838, 193, 198.

Leptocephalus Scopoli, 1777, 41 (22).

Ophisoma Swainson, 1839, 205.

Oxyurus Rafinesque, 1810, 79. 
Congrus Richardson, 1844, 223.

Helmichthys Costa, 1854, 256.

Leptocephalichthys Bleeker, 1856, 267.

Hyoprorus Kölliker, 1853, 254.

Uroconger Kaup, 1856, 272.

Congermuræna Kaup, 1856, 272.

Congerodon Kaup, 1856, 271, 237.

Neoconger Girard, 1859, 291.

Hoplunnis Kaup, 1860, 297.

Gnathophis Kaup, 1860, 297.

Isognatha (Dekay) Gill, 1861, 303.

Diaphanichthys Peters, 1864, 334.

Oxyconger Bleeker, 1865, 335.

Pocilogconger Günther, 1871, 361.

Coloconger Alcock, 1889, 444.

Promyllantor Alcock, 1890, 449.
Xenomystax Gilbert, 1891, 454.

Euleptocephalus Strömman, 1895, 470.

Todarus Grassi \& Calandruccio, 1896, 471.

Scolenchelys Ogilby, 1897, 477.

Bathycongrus Ogilby, 1898, 483.

Congrellus Ogilby, 1898, 483.

Atopichthys Garman, 1899, 486.

Congrosoma Garman, 1899, 486.

Veternio Snyder, 1904, 511.

Metopomycter Gilbert, 1905, 513.

Microconger Fowler, 1912, 543.

Mayerina Silvester, 1915, 557.

Pseudophichthys Roule, 1915, 557.

Rhechias $^{102}$ Jordan, 1921.

Brachyconger ${ }^{182}$ Regan, 1922.

Endeconger ${ }^{10 \%}$ Jordan, 1923.

\footnotetext{
${ }^{16}$ Usually called Congride; Leptocephalus, an earlier name than Conger, is the larval form of Conger, but the name has been long used for larval eels in general.

${ }^{10}$ Proc. U.S. Nat. Mus., $59: 644$; orthotype R. armiger Jordan.

103: Two new fishes from New Britain and Japan: Ann. Mag. Nat. Hist. X. 217; orthotype B. platyrhynchus, Regan; not of Bleeker, 1865.

${ }^{100}$ New name for Brachyconger, preoccupied.
}

\section{Family 212. NETTASTOMID王}

\section{(Duck-bill Eels)}

Nettastoma Rafinesque, 1810, 81.

Chlopsis Rafinesque, 1810, 82.

Saurenchelys Peters, 1864, 334.
Nettastomops Steindachner, 1906, 521.

Nettastomella Facciolà, 1914, 353.

Venefica Jordan \& Davis, 1892, 458.

\section{Family 213. HETEROCONGRID $\mathbb{E}$}

Heteroconger Bleeker, 1868, 348.

Tæniconger ${ }^{163}$ Herre, 1923.

Herre, "Preliminary Account of Philippine Eels" (in press); body ribbonlike, the depth eighty-six times in length; pectorals present.

\section{Family 214. ENCHELIID $\approx$}

Enchelion Hay, 1903, 503.

\section{Family 215. URENCHELYID在}

URENCHELYS Woodward, 1900, 491.

\section{Family 216. ECHELID屟 (Myrida)}

Echelus Rafinesque, 1810, 81 .

ENCHELyopus Agassiz, 1844, 181, 218.

Myrophis Lütken, 1851, 249.

Chilorhinus Lütken, 1851, 249.

Murænichthys Bleeker, 1853, 252.

Myrus Kaup, 1856, 271.

Paranguilla Bleeker, 1865, 335.

Paramyrus Günther, 1870, 357.

Holopterura Cope, 1871, 361.

Bathymyrus Alcock, 1890, 450.
Ahlia Jordan \& Davis, 1892, 458.

Verma Jordan \& Evermann, 1896, 473.

Myropterura Ogilby, 1897, 477.

Eom yrus Storms, 1898, 484.

RHYNCHORHINUS (Agassiz) Woodward, 1901, 219, 496.

Eomyrus (Agassiz) Eastman, 1905, 512.

Mylomyrus Woodward, 1910, 537.

Garmannichthys Seale, 1917, 563. 


\section{Family 217. NEMICHTHYID $\approx$}

(Thread Eels)

Nemichthys Richardson, 1848, 240.

Leptorhynchus Lowe, 1852, 251.

Belonopsis Brandt, 1854, 256.

Labichthys Gill \& Ryder, 1883, 424.

Spinivomer G. \& R., 1883, 424.

Serrivomer G. \& R., 1883, 424.
Gavialiceps Alcock, 1889, 444.

Avocettina Jordan \& Davis, 1892, 458.

Nematoprora Gilbert, 1905, 513.

Stemonidium Gilbert, 1905, 513.

Ceromitus Weber, 1913, 551.

\section{Family 218. CYEMID㤅}

Cyema Günther, 1878, 395.

\section{Family 219. OPHICHTHYID死}

Ophichthus $^{164}$ Ahl, 1787, 45.

Cocula Vahl, 1794, 52.

Sphagebranchus Bloch, 1795, 53.

Ophisurus Lacépède, 1800, 56.

Cæcilia Lacépède, 1800, 56.

Colubrina ${ }^{165}$ Lacépède, 1803, 65.

Apterichthys Duméril, 1806, 75.

Oxystomus Rafinesque, 1810, 83.

Dalophis Rafinesque, 1810, 81.

Cogrus Rafinesque, 1810, 81.

Pterurus Rafinesque, 1810, 82.

Helmictis Rafinesque, 1810, 83.

Typhlotes Fischer, 1813, 83.

Branderius Rafinesque, 1815, 92.

Leptognathus Swainson, 1839, 205.

Ophisternon McClelland, 1844, 220.

Ptyobranchus- McClelland, 1844, 220.

Ophithorax McClelland, 1844, 220.

Leptorhynchus ${ }^{105 \mathrm{n}}$ Smith, 1847, 244.

Anepistomon Gistel, 1848, 236.

Stethopterus Bleeker, 1853, 252.

Leiuranus Bleeker, 1853, 253.

Leiurus Kaup, 1856, 268.

Scytallurus Kaup, 1856, 268.

Centrurophis Kaup, 1856, 271.

Pœcilocephalus Kaup, 1856, 271.

\section{(Snake Eels)}

Microdonophis Kaup, 1856, 271.

Cocilophis Kaup, 1856, 271.

Herpetoichthys Kaup, 1856, 271.

Elapsopsis Kaup, 1856, 271.

Brachysomophis Kaup, 1856, 271.

Mystriophis Kaup, 1856, 271.

Murcenopsis $^{\text {1esb }}$ Kaup, 1856, 271.

Echiopsis Kaup, 1856, 271, 297.

Scytalophis Kaup, 1856, 271.

Leptorhinophis Kaup, 1856, 271.

Lamnostoma Kaup, 1856, 271.

Pisodonophis $^{168}$ Kaup, 1856, 271.

Ophisurapus Kaup, 1856, 271.

Cirrhimuræna Kaup, 1856, 271.

Callechelys Kaup, 1856, 271.

Anguisurus Kaup, 1856, 271.

Myrichthys Girard, .1859, 290.

Crotalopsis $^{187}$ Kaup, 1860, 297.

Cryptopterus Kaup, 1860, 297.

Achirophichthys Bleeker, 1865, 335 .

Uranichthys Poey, 1867, 347.

Macrodonophis Poey, 1867, 347.

Letharchus Goode \& Bean, 1882, 419.

Bascanichthys Jordan \& Davis, 1892, 456.

Quassiremus J. \& D., 1892, 458.

Scytalichthys J. \& D., 1892, 458.

${ }^{104}$ Usually written Ophichthys.

${ }^{105}$ Probably fictitious; eel-shaped, long-nosed, with snake-like scales on head; abdominal ventrals, no dorsal; tail forked.

${ }^{165 a}$ Leptorhynchus must be prior to its substitute Anepistomon.

${ }^{105 b}$ Murcenopsis as used by Kaup is a simple misprint for Murcenophis.

${ }^{106}$ Written also Pisoodontophis.

${ }^{107}$ Misprinted Crotalopis in "Genera of Fishes." 
Xyrias Jordan \& Snyder, 1901, 494.

Chlevastes J. \& S., 1901, 494.

Jenkinsiella Jordan \& Evermann, 1905, 576.
Hemerorhinus Weber \& Beaufort, 1916 , 561.

Omochelys ${ }^{188}$ Fowler, 1918.

Syletor Jordan, 1919, 567.

Acanthenchelys $^{168 n}$ Regan, 1922

${ }^{168}$ Proc. Acad. Nat. Sci. Phila., 8, 1818; orthotype Pisodonophis cruentifer Goode \& Bean. Same as Syletor Jordan, 1920; preoccupied in beetles (Syletor Tchitcherine, 1899).

108a "A new Eel from Tobago." Ann. Mag. Nat. Hist. X. 296; orthotype A. spinicauda Regan.

\section{Family 220. NEENCHELYID $\approx$}

Neenchelys Bamber, 1915, 555.

\section{Family 221. MORINGUID E}

Rataboura Gray, 1831, 138.

Moringua Gray, 1831, 138.

Pseudomoringua Bleeker, 1865, 335.

Pterurus Swainson, 1839, 205.

Pachyurus Swainson, 1839, 205.

Ptyobranchus McClelland, 1844, 220.

Aphthalmichthys Kaup, 1856, 272.

Stilbiscus Jordan \& Bollman, 1888, 442.

Gordiichthys Jordan \& Davis, 1892, 458.

Cryptophthalmus Franz, 1910, 535.

Unagius Jordan, 1919, 567.

\section{Suborder COLOCEPHALI}

\section{Family 222. HETERENCHELYID压}

Heterenchelys Regan, 1912, $545 . \quad$ Panturichthys Pellegrin, 1913, 549.

\section{Family 223. MURÆNIDÆ}

(Morays)

Muræna Linnæus, 1758, 11.

Echidna Forster, 1777, 42.

Gymnothorax Bloch, 1795, 53.

Murænophis Cuvier, 1798, 54.

Murænophis Lacépède, 1803, 68.

Gymnomuræna Lacépède, 1803, 68.

Megaderus Rafinesque, 1815, 92.

Gymnopsis Rafinesque, 1815, 92.

Haliophis Rüppell, 1828, 122.

Ichthyophis Lesson, 1828, 123.

Uropterygius Rüppell, 1835, 184.

Strophidon McClelland, 1844, 220, 574.

Lycodontis McClelland, 1844, 220.

Thærodontis McClelland, 1844, 220.

Channo-Muræna Richardson, 1844, 223.

Molarii ${ }^{189}$ Richardson, 1844, 223.

Ichthyapus Brisout de Barneville, 1847, 231.
Enchelynassa Kaup, 1855, 265, 271.

Enchelycotte Kaup, 1856, 268.

Eurymyctera Kaup, 1856, 271.

Siderea Kaup, 1856, 271.

Enchelycore Kaup, 1856, 271.

Thyrsoidea Kaup, 1856, 271.

Limamuræna Kaup, 1856, 271.

Polyuranodon Kaup, 1856, 272.

Pœcilophis Kaup, 1856, 272.

Murænoblenna Kaup, 1856, 272.

Neomuræna Girard, 1859, 291.

Priodonophis Kaup, 1860, 297.

Tæniophis Kaup, 1860, 297.

Pseudomuræna Johnson, 1862, 319.

Pseudechidna Bleeker, 1863, 322.

Pythonichthys Poey, 1867, 347.

Rhinomuræna Garman, 1888, 441.

Rabula Jordan \& Davis, 1892, 458.

${ }^{100}$ In plural form only. 
Scutica Jordan \& Evermann, 1896, 473. Emasia Jordan \& Snyder, 1901, 494.

Scuticaria J. \& S., 1901, 494.

Evenchelys Jordan \& Evermanin, 1902, 498.
Anarchias Jordan \& Seale, 1906, 519.

Rhabdura Ogilby, 1907, 526.

Rhinechidna Barbour, 1908, 527.

Ahynnodontophis Fowler, 1912, 543.

Deprandus ${ }^{170}$ Jordan \& J. Z. Gilbert, 1921.

170 "Fish Fauna California Tertiary," 252; orthotype D. lestes Jordan \& Gilbert.

\title{
Suborder LYOMERI
}

Family 224. SACCOPHARYNGID压

\author{
(Gulpers)
}

Saccopharynx Mitchill, 1824, $117 . \quad$ Ophiognathus Harwood, 1827, 121.

\section{Family 225. EURYPHARYNGID五}

(Swallowers)

According to Dr. Roule, the nominal genera of this group are all identical with Eurypharynx.

Eurypharynx Vaillant, 1882, 421.

Gastrostomus Gill \& Ryder, 1883, 424.
Megalopharynx Brauer, 1900, 489.

Macropharynx Brauer, 1902, 497.

\section{Series OSTARIOPHYSI}

(Plectospondyli)

\section{Order HETEROGNATHI}

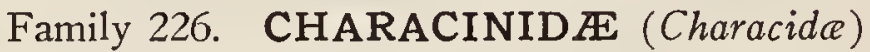

(Characins)

Some of the genera named below are perhaps referable to other families.

Characinus ${ }^{171}$ Linnæus, 1758, 14.

Charax Gronow, 1763, 21.

Erythrinus Gronow, 1763, 21.

Charax (Gronow) Scopoli, 1777, 42.

Erythrinus (Gronow) Scopoli, 1777, 42.

Serrasalmus ${ }^{172}$ Lacépède, 1803, 66.

Characinus Lacépède, 1803, 66.

Tetragonopterus Cuvier, 1815, 93.

Myletes Cuvier, 1815, 93.

Chalceus Cuvier, 1817, 96.

Hydrocyon Cuvier, 1817, 96.

Characinus Cuvier, 1817, 98.

Piabucus $^{172}$ (Cuvier) Oken, 1817, 98.

Erythrinus Agassiz, 1829, 173.

Salminus Agassiz, 1829, 132:
Xiphorhynchus Agassiz, 1829, 132.

Schizodon Agassiz, 1829, 132.

Cynodon $^{174}$ Spix, 1829, 132.

Rhaphiodon Agassiz, 1829, 132.

Erythrichthys Bonaparte, 1833, 175.

Macrodon Müller, 1843, 216.

Myleus Müller \& Troschel, 1844, 221.

Alestes M. \& T., 1844, 221.

Catoprion M. \& T., 1844, 221.

Pygocentrus M. \& T., 1844, 221.

Pygopristis M. \& T., 1844, 221.

Epicyrtus M. \& T., 1844, 221.

Xiphorhamphus M. \& T., 1844, 221.

Hydrolycus M. \& T., 1844, 221.

Brycon M. \& T., 1844, 221.

\footnotetext{
${ }^{1 r 1}$ In plural form only, as Characini.
}

${ }^{172}$ Written Serrasalmo by Cuvier.

${ }^{173}$ Written Piabuca by authors.

${ }^{174}$ The dates of birth and death of Spix are incorrectly given in "Genera of Fishes," p. 173; read 1781-1826. 
Exodon M. \& T., 1844, 221.

Cnidon M. \& T., 1845, 227.

Agoniates M. \& T., 1845, 227.

Grundulus Cuvier \& Valenciennes, 1846, 228.

Lebiasina Cuv. \& Val., 1846, 229.

Pyrrhulina Cuv. \& Val., 1846, 229.

Hydropardus Reinhardt, 1849, 244.

Cynopotamus Cuvier \& Valenciennes, 1849, 242.

Chalcinus Cuv. \& Val., 1849, 242.

Mylesinus Cuv. \& Val., 1849, 242.

Tometes Cuv. \& Val., 1849, 242.

Piabucina Cuv. \& Val., 1849, 242.

Brycinus Cuv. \& Val., 1849, 242.

Astyanax Baird \& Girard, 1854, 255.

Cheirodon $^{175}$ Girard, 1854, 258.

Stevardia Gill, 1858, 281.

Corynopoma Gill, 1858, 281.

Nematopoma Gill, 1858, 281.

Pœcilurichthys Gill, 1858, 281.

Hemigrammus Gill, 1858, 281.

Bryconops Kner, 1859, 292.

Pseudochalceus $^{176}$ Kner, 1863, 327.

Chalcinopsis $^{17 \tau}$ Kner, 1863, 337.

Crenuchus Günther, 1863, 326.

B rachyalestes Günther, 1864, 333.

Hetererythrinus Günther, 1864, 332.

Hemibrycon Günther, 1864, 333.

Scissor Gïnther, 1864, 333.

Creatochanes Günther, 1864, 333.

Creagrutus Günther, 1864, 333.

Anacyrtus Günther, 1864, 333.

Rœstes Günther, 1864, 333.

Rœboides Günther, 1864, 333.

Hystricodon Günther, 1864, 333.

Sarcodaces Günther, 1864, 333.

Oligosarcus Günther, 1864, 333.

Psalidostoma $^{178}$ Kner, 1864, 338.

Piabina Reinhardt, 1866, 343.

Aphyocharax Günther, 1868, 351.

Megalobrycon Günther, 1869, 504.

Holotaxis Cope, 1870, 356.

Odontostilbe Cope, 1870, 356.
Stethaprion Cope, 1870, 356.

Plethodectes Cope, 1870, 356.

Proportheus Cope, 1872, 363.

Triportheus Cope, 1872, 363.

Iguanodectes Cope, 1872, 363.

Bryconæthiops Günther, 1873, 369.

Paragoniates Steindachner, 1876, 387.

Lutkenia Steindachner, 1876, 387.

Metynnis Cope, 1878, 394.

Bramocharax Gill, 1877, 389.

Leptogoniates Boulenger, 1887, 437.

Henochilus Garman, 1890, 451.

Pseudocorynopoma Perugia, 1891, 455.

Chalcinopelecus Holmberg, 1891, 455.

Bergia Steindachner, 1891, 456.

Brachychalcinus Boulenger, 1892, 457.

Asiphonichthys Cope, 1894, 464.

Diapoma Cope, 1894, 464.

Petersius Hilgendorf, 1894, 465.

Myloplus Gill, 1895, 467.

Hoplerythrinus Gill, 1895, 467.

Micralestes Boulenger, 1899, 485.

Neoborus Boulenger, 1899, 485.

Catabasis Eigenmann \& Norris, 1900, 489.

Orthomyleus Eigenmann, 1903, 502.

Colossoma Eigenmann, 1903, 502.

Mylossoma Eigenmann, 1903, 502.

Boulengerella Eigenmann, 1903, 502.

Acnodon Eigenmann, 1903, 502.

Myleocollops Eigenmann, 1903, 502.

Gilbertella Eigenmann, 1903, 502.

Evermannella Eigenmann, 1903, 502.

Acestrocephalus Eigenmann, 1903, 502.

Holoshesthes $^{170}$ Eigenmann, 1903, 502.

Mœnkhausia Eigenmann, 1903, 502.

Markiana Eigenmann, 1903, 502.

Othonophanes Eigenmann, 1903, 502.

Holoprion Eigenmann, 1903, 502.

Holopristis Eigenmann, 1903, 502.

Stichonodon Eigenmann, 1903, 502.

Bryconodon Eigenmann, 1903, 502.

Acestrorhynchus Eigenmann, 1903, 502.

Piaractus Eigenmann, 1903, 502.

\footnotetext{
${ }^{175}$ Often corrected to Chirodon.

${ }^{176}$ This name first appears in Sitzb. Acad. Wiss. München, 226, 1863.

${ }^{177}$ Kner, Sitzb. Acad. Wiss. München, 225, 1863.

${ }^{178}$ First published in 1864.

${ }^{170}$ Later written, more correctly, Holesthes.
} 
Acestrorhamphus Eigenmann, 1903, 502.

Gymnocharacinus Steindachner, 1903, 506.

Hoplias Gill, 1903, 503.

Eucynopotamus Fowler, 1904, 507.

Copeina Fowler, 1906, 518.

Ophiocephalops Fowler, 1906, 518.

Cyphocharax Fowler, 1906, 518.

Steindachnerina Fowler, 1906, 518.

Peltapleura Fowler, 1906, 518.

Phoxinopsis Regan, 1907, 526.

Mimagoniates Regan, 1907, 526.

Ctenocharax Regan, 1907, 526.

EOBRYCON Jordan, 1907, 525.

Pellegrinina Fowler, 1907, 523.

Coscinoxyron Fowler, 1907, 523.

Cynocharax Fowler, 1907, 523.

Cyrtocharax Fowler, 1907, 523.

Sphyrænocharax Fowler, 1907, 523.

Belonocharax Fowler, 1907, 523.

Waiteina Fowler, 1907, 523.

Reganina Fowler, 1907, 523.

Starksina Fowler, 1907, 523.

Sealeina Fowler, 1907, 523.

Bryconamericus Eigenmann, 1907, 522.

Gilbertolus Eigenmann, 1907, 522.

Evermannolus Eigenmann, 1907, 522.

Phenacogrammus Eigenmann, 1907, 522.

Phenacogaster Eigenmann, 1907, 522.

Thayeria Eigenmann, 1907, 527.

Deuterodon Eigenmann, 1907, 522.

Astyacinus Eigenmann, 1907, 522.

Fowlerina Eigenmann, 1907, 522.

Joinvillea Steindachner, 1908, 531.

Pristella Eigenmann, 1908, 528.

Poptella Eigenmann, 1908, 528.

Psellogrammus Eigenmann, 1908, 528.

Thayeria Eigenmann, 1908, 527.

Ctenobrycon Eigenmann, 1908, 527.

Cœlurichthys Ribeiro, 1908, 530.

Gymnocorymbus Eigenmann, 1908, 527.

Brycochandus Eigenmann, 1908, 528.

Hyphessobrycon Eigenmann, 1908, 528.

Champsoborus Boulenger, 1909, 531.

Dermatocheir Durbin, 1909, 532.

Triurobrycon Eigenmann, 1909, 532.

Holobrycon Eigenmann, 1909, 532.

Pterodiscus Eigenmann, 1909, 532.

Pœcilocharax Eigenmann, 1909, 533.
Hollandichthys Eigenmann, 1910, 535.

Rhoadsia Fowler, 1911, 539.

Probolodus Eigenmann, 1911, 538.

Psalidodon Eigenmann, 1911, 538.

Spintherobolus Eigenmann, 1911, 538.

Glandulocauda Eigenmann, 1911, 538.

Vesicatrus Eigenmann, 1911, 538.

Hysteronotus Eigenmann, 1911, 538.

Nematobrycon Eigenmann, 1911, 538.

Hasemania Ellis, 1911, 538.

Apodastyanax Fowler, 1911, 539.

Knodus Eigenmann, 1911, 538.

Genycharax Eigenmann, 1912, 543.

Gephyrocharax Eigenmann, 1912, 543.

Acanthocharax Eigenmann, 1912, 543.

Heterocharax Eigenmann, 1912, 543.

Aphyodite Eigenmann, 1912, 542.

Tylobranchus Eigenmann, 1912, 542.

Bivibranchia Eigenmann, 1912, 542.

Parastremma Eigenmann, 1912, 543.

Tyttocharax Fowler, 1913, 548.

Apístogramma Regan, 1913, 550.

Xenurocharax Regan, 1913, 550.

Ephippicharax Fowler, 1913, 548.

Gnathocharax Fowler, 1913, 548.

Pterobrycon Eigenmann, 1913, 547.

Prionobrama Fowler, 1913, 548.

Argopleura Eigenmann, 1913, 547.

Microgenys Eigenmann, 1913, 547.

Zygogaster Eigenmann, 1913, 547.

Ceratobranchia Eigenmann, 1914, 553.

Phanagoniates Eigenmann \& Henn, 1914, 553.

Landonia E. \& H., 1914, 553.

Parecbasis Eigenmann, 1914, 553.

Bleptonema Eigenmann, 1914, 553.

Microbrycon Eigcnmann, 1914, 553.

Xiphocharax Fowler, 1914, 553.

Carlia Meek, 1914, 554.

Gastropristis Eigenmann, 1915, 556.

Rooseveltiella Eigenmann, 1915, 556.

Pristobrycon Eigenmann, 1915, 556.

Mixobrycon Eigenmann, 1915, 556.

Compsura Eigenmann, 1915, 556.

Aphyocheirodon Eigenmann, 1915, 556.

Oligobrycon Eigenmann, 1915, 555.

Microschemobrycon Eigenmann, 1915, 555.

Megalamphodus Eigenmann, 1915, 555. 
Macropsobrycon Eigenmann, 1915, 555. Leptobrycon Eigenmann, 1915, 555.

Equidens Steindachner, 1915, 558.

Pseudocheirodon Meek \& Hildebrand, 1916, 559.
Entomolepis Eigenmann, 1917, 561.

Bertoniolus ${ }^{180}$ Fowler, 1918.

Characilepis Cockerell, 1920, 570.

Phenacobrycon $^{181}$ Eigenmann, 1920.

${ }^{180}$ "A New Characin from Paraguay," Proc. Acad. Nat. Sci. Phila., 141, 1918; orthotype $B$. paraguayensis Fowler.

${ }^{181}$ Eigenmann, Indiana University Studies, 45: 18; orthotype Bryconamericus henni Eigenmann.

\section{Family 227. GASTEROPELECID $\mathbb{E}$}

Gasteropelecus Gronow, 1763, 22.

Gasteropelecus Pallas, 1769, 22.

Thoracocharax Fowler, 1907, 523.

Carnegiella Eigenmann, 1909, 532.

\section{Family 228. XIPHOSTOMID王}

Xiphostoma Agassiz, 1829, 132.

Luciocharax Steindachner, 1876, 387.

Ctenolucius Gill, 1861, 302.

\section{Family 229. ANOSTOMIDIE (Curimatidce: Anodontidce)}

Anostomos Gronow, 1763, 20.

Anostomus (Gronow) Scopoli, 1777, 42.

Curimata Walbaum, 1792, 50.

Anostomus (Gronow) Cuvier, 1817, 98.

Curimatus (Cuvier) Oken, 1817, 98.

Leporinus Spix, 1829, 132.

Prochilodus Agassiz, 1829, 132.

Pacu Spix, 1829, 132.

Anodus Spix, 1829, 132.

Mormyrhynchus Swainson, 1839, 203.

Chilodus Mitiller \& Troschel, 1844, 221.

Histiodromus Gistel, 1848, 234.

Microdus Kner, 1859, 292.

Rhytiodus Kner, 1859, 292.

Cænotropus Günther, 1864, 333.

Læmolyta Cope, 1872, 363.

Leporellus Lütken, 1874, 376.
Curimatopsis Steindachner, 1876, 387.

Elopomorphus Gill, 1878, 394.

Potamorhina Cope, 1878, 394.

Psectrogaster Eigenmann \& Eigenmann, $1889,445$.

Curimatella ${ }^{181 a}$ E. \& E., 1889, 446.

Semitapicis E. \& E., 1889, 446.

Schizodontopsis Garman, 1890, 451.

Lahiliella Eigenmann, 1903, 502.

Pithecocharax Fowler, 1906, 518.

Chilomyzon Fowler, 1906, 518.

Eigenmannina Fowler, 1906, 518.

Garmanina Fowler, 1906, 518.

Abramites Fowler, 1906, 518.

Peltapleura Fowler, 1906, 518.

Anostomoides Pellegrin, 1908, 529, 533

Xyrocharax Fowler, 1913, 547.

${ }^{181 a}$ Curimatella Pellégrin is introduced by error on page 533.

\section{Family 230. HEMIODONTID $\mathbb{E}$}

Hemiodus Müller, 1843, 216, 243.

Parodon Cuvier \& Valenciennes, 1849, 242.

Centrophorus Kner, 1859, 292.

Characidium Reinhardt, 1866, 343.

Saccodon ${ }^{182}$ Kner, 1863, 327.

Nannostomus Günther, 1872, 365.

Chorimycterus Cope, 1894, 464.

Nanognathus Boulenger, 1895, 466.
Anisistia Eigenmann, 1903, 502.

Pocilosomatops Fowler, 1906, 518.

Hemiodopsis Fowler, 1906, 518.

Jobertina Pellegrin, 1909, 533.

Pocilobrycon Eigenmann, 1909, 533.

Archicheir Eigenmann, 1909, 533.

Microcharax Eigenmann, 1909, 533

Apareiodon Eigenmann, 1916, 559. Kner.

${ }^{182}$ Kner, Sitzb. Acad. Wiss. München, 225, 1863; orthotype Saccodon wagneri 


\section{Family 231. CITHARINID $\#$}

Citharinus $^{183}$ (Cuvier) Oken, 1817, 98, 243.

Distichodus Müller \& Troschel, 1845, 227, 242.

Ichthyborus ${ }^{184}$ Günther, 1864, 333.

Phago Günther, 1865, 337.

Xenocharax Günther, 1867, 345.

Nannocharax Günther, 1867, 345.

Nannæthiops Günther, 1871, 361.

Monostichodus Vaillant, 1900, 491.
Hemistichodus Pellegrin, 1900, 491, 557.

Neolebias Steindachner, 1894, 465.

Eugnathichthys Boulenger, 1898, 479.

Paraphago Boulenger, 1898, 485.

Neoborus Boulenger, 1898, 485.

Mesoborus Pellegrin, 1900, 490.

Citharidium Boulenger, 1902, 497.

Microstomatichthyoborus Nichols \& Griscom, 1917, 562.

Paradistichodus ${ }^{185}$ Pellegrin, 1922.

${ }^{183}$ The original spelling, Cytharinus, may be a lapse for Citharinus, as Cuvier wrote "les Citharines" and Valenciennes "Citharinus."

${ }^{184}$ Not Ichthyoborus Kaup, 1845, a genus of birds.

188 "Poissons de l'Oubranchi-Chari," Bull. Zool. Soc. France, 47: 70; orthotype Nannocharax dimidatus Pellegrin.

\section{Order GYMNONOTI}

\section{Family 232. RHAMPHICHTHYIDE}

Rhamphichthys Müller \& Troschel, 1849, 243.

Altona Kaup, 1856, 268.

Hypopomus Gi11,.1864, 330 .

Brachyrhamphichthys Günther, 1870 , 357.

\section{Family 233. APTERONOTID开 (Sternarchida)}

Apteronotus Lacépède, 1800, 56.

Sternarchus Bloch \& Schneider, 1801, 59.

Carapus $^{188}$ Rafinesque, 1810, 82.

Sternarchorhynchus Castlenau, 1855, 264.

Memarchus Kaup, 1856, 268.

Rhamphosternarchus Günther, 1870 , 357.

${ }^{188}$ The final disposition of this generic name is open to question. The decision of the International Commission removes it from this group to replace Fierasfer Cuvier.

\section{Family 234. GYMNOTID $\mathbb{E}$}

Gymnotus, Linnæus, 1758, 12.

Carapus Cuvier, 1817, 101, 243.

Carapo Oken, 1817, 101.

Sternopygus Müller \& Troschel, 1849, 243.
(Carapos)

Giton Kaup, 1856, 268.

Gymnotes Gill, 1864, 330.

Cryptops Eigenmann, 1894, 464.

Eigenmannia Jordan \& Evermann, 1896, 473.

\section{Family 235. ELECTROPHORID IE}

(Electric Eels)

Gymnotus Linnæus, 1766 (not of 1758). Electrophorus Gill, 1864, 330. 


\section{Order EVENTOGNATHI}

\section{Family 236. CATOSTOMID压}

(Suckers; Buffalo Fishes)

Catostomus Le Sueur, 1817, 96.

Hypentelium Rafinesque, 1818, 109.

Cycleptus Rafinesque, 1819, 110.

Ictiobus $^{187}$ Rafinesque, 1820, 112.

Carpiodes Rafinesque, 1820, 112.

Moxostoma ${ }^{188}$ Rafinesque, 1820, 112.

Decactylus Rafinesque, 1820, 113.

Teretulus Rafinesque, 1820, 113.

Eurystomus $^{189}$ Rafinesque, 1820, 113.

Stomocatus Bonaparte, 1840, 206.

Sclerognathus Cuvier \& Valenciennes, 1844, 219.

Hylomyzon Agassiz, 1855, 261.

Ptychostomus Agassiz, 1855, 261.

Bubalichthys Agassiz, 1855, 261.

Acomus Girard, 1856, 269.

Minomus Girard, 1856, 269.
Placopharynx Cope, 1870, 356.

Amyzon Cope, 1872, 364.

Pantosteus Cope, 1876, 384.

Erimyzon Jordan, 1876, 386.

Lagochila Jordan \& Brayton, 1877, 391.

Myxocyprinus Gill, 1878, 394.

Minytrema Jordan, 1878, 395.

Chasmistes Jordan, 1878, 396.

Quassilabia Jordan \& Brayton, 1878, 395.

Lipomyzon Cope, 1881, 415.

Xyrauchen Kirsch, 1888, 443.

Deltistes Seale, 1896, 475.

Megastomatobus Fowler, 1913, 547.

Pithecomyzon Fowler, 1913, 548.

Scartomyzon Fowler, 1913, 548.

Notolepidomyzon Fowler, 1913, 548.

Thoburnia Jordan \& Snyder, 1917, 562.

${ }^{187}$ Spelled Ichthyobus by authors.

${ }^{188}$ Name corrected to Myxostoma.

${ }^{189}$ Mythical.

Family 237. CYPRINID压

(Carps, Dace, Minnows, Chubs, etc.)

Cyprinus Linnæus, 1758, 15.

Leuciscus Klein, 1775, 37.

Brama Klein, 1775, 37.

Mystus Klein, 1775, 38.

Labeo Cuvier, 1817, 99.

Tinca Cuvier, 1817, 99.

Gobio Cuvier, 1817, 99.

Abramis Cuvier, 1817, 99.

Barbus Cuvier, 1817, 99.

Leuciscus Cuvier, 1817, 99.

Cirrhinus $^{100}$ (Cuvier) Oken, 1817, 99.

Exoglossum Rafinesque, 1818, 109.

Maxillingua Rafinesque, 1818, 109.

Notropis Rafinesque, 1818, 108.

Notemigonus Rafinesque, 1819, 110.

Hemiplus Rafinesque, 1820, 110.

Minnilus Rafinesque, 1820, 111.

Dobula Rafinesque, 1820, 112.

Phoxinus Rafinesque, 1820, 112.

Alburnus Rafinesque, 1820, 112.

Luxilus Rafinesque, 1820, 112 :
Chrosomus Rafinesque, 1820, 112.

Semotilus Rafinesque, 1820, 112.

Rutilus Rafinesque, 1820, 112.

Pimephales Rafines๋que, $1820,112$.

Plargyrus Rafinesque, 1820, 112.

Garra Hamilton, 1822, 115.

Morulius Hamilton, 1822, 115.

Cabdio Hamilton, 1822, 115.

Puntius Hamilton, 1822, 115.

Barilius Hamilton, 1822, 115.

Bangana Hamilton, 1822, 115.

Chela Hamilton, 1822, 115.

Danio Hamilton, 1822, 115.

Diplocheilus Van Hasselt, 1823, 172.

Crossocheilus Van Hasselt, 1823, 116.

Lobocheilus Van Hasselt, 1823, 116.

Hampala Van Hasselt, 1823, 116.

Nandina Gray, 1831, 138.

Carassius Nilsson, 1832, 176.

Bengala Gray, 1833, 139, 179.

Tor Gray, 1833, 139, 179.

\footnotetext{
${ }^{100}$ Spelled Cirrhina by authors.
} 
Phoxinus Agassiz, 1835, 180.

Rhodeus Agassiz, 1835, 180.

Chondrostoma Agassiz, 1835, 180.

Aspius Agassiz, 1835, 180.

Pelecus Agassiz, 1835, 180.

Varicorhinus Rüppell, 1836, 186.

Labeobarbus Rüppell, 1836, 186.

Squalius Bonaparte, 1837, 142, 187.

Telestes Bonaparte, 1837, 142, 187.

Scardinius Bonaparte, 1837, 142, 187.

Cyprinopsis Bonaparte, 1837, 142, 187.

Schizothorax Heckel, 1838, 192.

Platycara McClelland, 1839, 195.

Gonorhynchus McClelland, 1839, 195.

Oreinus McClelland, 1839, 195.

Systomus McClelland, 1839, 195.

Perilampus McClelland, 1839, 195.

Opsarius McClelland, 1839, 195.

Esomus Swainson, 1839, 202.

Chedrus Swainson, 1839, 202.

Salmophasia Swainson, 1839, 202.

Salmostoma Swainson, 1839, 198.

Phoxinellus Heckel, 1840, 211, 288.

Aulopyge Heckel, 1841, 209.

Rohtee Sykes, 1841, 210.

Racoma McClelland, 1842, 212.

Stilbe Dekay, 1842, 210.

Capoëta Cuvier \& Valenciennes, 1842, 210.

Rohita Cuv. \& Val., 1842, 210.

Nuria Cuv. \& Val., 1842, 210.

Dangila Cuv. \& Val., 1842, 210.

Tylognathus Heckel, 1842, 211.

Discognathus Heckel, 1842, 211.

Luciobarbus Heckel, 1842, 211.

Leucosomus Heckel, 1842, 211.

Argyreus Heckel, 1842, 211

Pachystomus Heckel, 1842, 211.

Osteobrama Heckel, 1842, 211.

Acanthobrama Heckel, 1842, 211.

Ballerus Heckel, 1842, 211.

Chondrorhynchus Heckel, 1842, 211.

Chondrochilus Heckel, 1842, 211.

Gymnostomus Heckel, 1842, 211.

Scaphiodon Heckel, 1842, 211.

Dillonia Heckel, 1842, 211.

Cyprinion Heckel, 1842, 211.
Gibelion Heckel, 1842, 211.

Soricidens Münster, 1842, 212.

CApitodus Münster, 1842, 212.

Aspidoparia Heckel, 1843, 215.

Schizopyge Heckel, 1843, 215.

Cyrene Heckel, 1843, 215.

Mola Heckel, 1843, 215.

Leucos Heckel, 1843, 215.

Catla Cuvier \& Valenciennes, 1844, 219.

Cenisophius Bonaparte, 1845, 226.

Gardonus Bonaparte, 1845, 226.

Pigus Bonaparte, 1845, 226.

Cephalus Bonaparte, 1845, 226.

Microlepis Bonaparte, 1845, 226.

Hegerius Bonaparte, 1845, 226.

Rhinichthys Agassiz, 1848, 232.

Englottogaster Gistel, 1848, 236.

Cheilobarbus Smith, 1849, 244.

Pseudobarbus Smith, 1849, 244.

Abrostomus Smith, 1849, 244.

Hypsolepis $^{191}$ Baird, 1850, 245, 255.

Gila Baird \& Girard, 1853, 252.

Ceratichthys $^{102}$ B. \& G., 1853, 252, 270.

Pogonichthys Girard, 1854, 257.

Hybopsis Agassiz, 1854, 254.

Cochlognathus Baird \& Girard, 1854, 255.

Lavinia Girard, 1854, 257.

TARSichthys Troschel, 1854, 261.

Cheilonemus Baird, 1855, 266.

Culter Basilewski, 1855, 262.

Nasus Basilewski, 1855, 262.

Cephalus Basilewski, 1855, 262.

Leptocephalus Basilewski, 1855, 262.

Luciosoma Bleeker, 1855, 264.

Epalzeorhynchos Bleeker, 1855, 264.

Schismatorhynchos Bleeker, 1855, 264.

Mylopharodon Ayres, 1855, 262.

Acrocheilus Agassiz, 1855, 261.

Campostoma Agassiz, 1855, 261.

Hyborhynchus Agassiz, 1855, 261.

Hybognathus Agassiz, 1855, 261.

Ptychocheilus Agassiz, 1855, 262.

Mylocheilus Agassiz, 1855, 262.

Opsaridium Peters, 1855, 409.

Agosia Girard, 1856, 269.

Nocomis Girard, 1856, 269.
${ }^{191}$ Later written Hypsilepis.

${ }^{192}$ Misprinted Ceraticthys. 
Cliola Girard, 1856, 269.

Alburnops Girard, 1856, 270.

Alburnellus Girard, 1856, 269.

Richardsonius Girard, 1856, 270.

Codoma Girard, 1856, 270.

Dionda Girard, 1856, 269.

Orthodon Girard, 1856, 269.

Algoma Girard, 1856, 269.

Algansea Girard, 1856, 269.

Clinostomus Girard, 1856, 270.

Hudsonius Girard, 1856, 270.

Tiaroga Girard, 1856, 270.

Tigoma Girard, 1856, 270.

Siboma Girard, 1856, 270.

Cyprinella Girard, 1856, 270.

Moniana Girard, 1856, 270.

Cheonda Girard, 1856, 270.

Blicca Heckel, 1858, 281.

Carpio Heckel, 1858, 281.

Lepidocephalus Bleeker, 1858, 279.

Idus Heckel, 1858, 281.

Leucaspis Heckel \& Kner, 1858, 281.

Balantiocheilos Bleeker, 1859, 287.

Lissorhynchus Bleeker, 1859, 287.

Acanthobrama Bleeker, 1859, 287.

Morara Bleeker, 1859, 287.

Macrochirichthys Bleeker, 1859, 288.

Laubuca Bleeker, 1859, 288.

Pseudophoxinus ${ }^{102 a}$ Bleeker, 1859, 288.

Hemibarbus Bleeker, 1859, 288.

Hypselobarbus Bleeker, 1859, 288.

Rohteichthys Bleeker, 1859, 288.

Barbodes Bleeker, 1859, 288.

Anematichthys Bleeker, 1859, 288.

Siaja Bleeker, 1859, 288.

Hemiculter Bleeker, 1859, 288.

Pseudoculter Bleeker, 1859, 288.

Gonoproktopterys Bleeker, 1859, 288.

Pseudogobio Bleeker, 1859, 287.

Smilogaster Bleeker, 1859, 287.

Opistocheilus Bleeker, 1859, 287.

Semiplotus Bleeker, 1859, 287.

Rohitichthys Bleeker, 1859, 287.

Barbichthys Bleeker, 1859, 287.

Discognathichthys Bleeker, 1859, 287.

Diplocheilichthys Bleeker, 1859, 287.

Chanodich thys Bleeker, 1859, 288.

Leptobarbus Bleeker, 1859, 288.
Trinematichthys Bleeker, 1859, 288.

Hypophthalmichthys Bleeker, 1859, 288.

Barbichthys Bleeker, 1859, 287.

Chrysophekadion Bleeker, 1859, 287.

Amblypharyngodon Bleeker, 1859, 288.

Devario Bleeker, 1859, 288.

Tambra Bleeker, 1859, 287.

Mrigala Blceker, 1859, 287.

Albulichthys Bleeker, 1859, 287.

Acheilognathus Bleeker, 1859, 287.

Amblyrhynchichthys Bleeker, 1859, 287.

Thynnichthys Bleeker, 1859, 288.

Elopichthys Bleeker, 1859, 288.

Shacra Bleeker, 1859, 288.

Bendilisis Bleeker, 1859, 288.

Pseudorasbora Bleeker, 1859, 288.

Rasbora Bleeker, 1859, 288.

Gnathopogon Bleeker, 1859, 288.

Rasborichthys Bleeker, 1859, 288.

Alburnoides Jeitteles, 1861, 308.

Bungia Keyserling, 1861, 308.

Owsianka Dybowski, 1862, 313.

Brama (Klein) Bleeker, 1862, 312.

Bliccopsis Siebold, 1863, 308.

Leptobarbus Bleeker, 1863, 321.

Acra Bleeker, 1863, 321.

Rohitodes Bleeker, 1863, 321.

Paraschizothorax Bleeker, 1863, 322.

Carpionichthys Bleeker, 1863, 322.

Abramidopsis Siebold, 1863, 328.

Isocephalus Heckel, 1863, 321.

Crossocheilichthys Bleeker, 1863, 321.

Gobionichthys Bleeker, 1863, 321.

Cirrhinichthys Bleeker, 1863, 321.

Opsariichthys Bleeker, 1863, 322.

Paraphoxinus Bleeker, 1863, 322.

Trachybrama Heckel, 1863, 322.

Paracheilognathus Bleeker, 1863, 322.

Pseudoperilampus Bleeker, 1863, 322.

Platygobio Gill, 1863, 323.

Peletrophus Günther, 1864, 333.

Scardiniopsis Jäckel, 1864, 334.

Pseudolaubuca Bleeker, 1865, 335.

Acanthobrama Bleeker, 1865, 335.

Paracanthobrama Bleeker, 1865, 335.

Parabramis Bleeker, 1865, 335.

Paralaubuca Bleeker, 1865, 335. 
Paradanio (Bleeker) Day, 1865, 337.

Brachygramma Day, 1865, 337.

Stilbius Gill, 1865, 337.

Ericymba Cope, 1865, 336.

Cyprinopsis Blanchard, 1866, 339.

Pachychilon Steindachner, 1866, 343.

Ctenopharyngodon Steindachner, 1866, 343.

Ptychobarbus Steindachner, 1866, 343.

Diptychus Steindachner, 1866, 343.

Schizopygopsis Steindachner, 1866, 343.

Phenacobius Cope, 1867, 344 .

Photogenis Cope, 1867, 344.

Enteromius Cope, 1867, 344.

Graodus Günther, 1868, 351.

Megarasbora Günther, 1868, 351.

Aphyocypris Günther, 1868, 351 .

Pteropsarion Günther, 1868, 351.

Mystacoleucus Günther, 1868, 351.

Opsaridium (Peters) Günther, 1868, 351.

Bola Günther, 1868, 351.

Schacra Günther, 1868, 351.

Ochetobius Günther, 1868, 351.

Squaliobarbus Günther, 1868, 351.

Eustira Günther, 1868, 351.

Securicula Günther, 1868, 351.

Cachius Günther, 1868, 351.

Oreonectes Günther, 1868, 351.

Gymnocypris Günther, 1868, 351.

Barynotus Günther, 1868, 351.

Xenocypsis Günther, 1868, 351.

Osteochilus Günther, 1868, 351.

Mayoa Day, 1869, 354.

Gobiobarbus Dybowski, 1869, 354.

Micraspius Dybowski, 1869, 354.

Pseudaspius Dybowski, 1869, 354.

Ladislavia Dybowski, 1869, 354.

Sarcocheilichthys Bleeker, 1869, 353.

Acrocheilichthys Bleeker, 1869, 353.

Abramocephalus Steindachner, 1869, 355.

Pseudobrama Bleeker, 1870, 355.

Luciobrama Bleeker, 1870, 355.

Rhinogobio Bleeker, 1870, 355.

Acanthorhodeus Bleeker, 1870, 355.

Saurogobio Bleeker, 1870, 355.
Hemitremia Cope, 1870, 356.

ANCHybopsis Cope, $1870,356$.

MyLOCYPRINUs Leidy, 1870, 359.

Lithichthys Cope, $1870,356$.

Diastichus Cope, $1870,356$.

Oligobelus Cope, 1870, 356.

Protoporus Cope, 1872, 363.

Myloleucus Cope, 1872, 363.

Sarcidium Cope, 1872, 363.

Coliscus Cope, 1872, 363.

Apocope Cope, 1872, 363.

Neocarassius ${ }^{102 b}$ Castelnau, 1872, 363 .

Gobiosoma Dybowski, 1872, 364 .

Onychodon Dybowski, 187., 364.

Barbodon Dybokski, 1872, 364.

Plagiognathus Dybowski, 1872, 364 .

Squalidus Dybowski, 1872, 364.

Megalobrama Dybowski, 1872, 364 .

Orfus Fitzinger, 1873, 369.

Zopa Fitzinger, 1873, 368.

Rubellus Fitzinger, 1873, 369.

Vimba Fitzinger, 1873, 368.

Habrolepis Fitzinger, 1873, 369.

Cephalopsis Fitzinger, 1873, 369.

Bathystoma Fitzinger, 1873, 369.

Machærochilus Fitzinger, 1873, 369.

Toxabramis Günther, 1873, 369.

Myloleucus Günther, 1873, 369.

Sinibarbus Sauvage, 1874, 376.

Eritrema Cope, 1876, 384.

Pristiodon Dybowski, 1877, 389.

Episema Cope \&.Jordan, 1877, 390.

Hemitrichas Peters, 1877, 392.

Aturius Dubalen, 1878, 394.

Cosmochilus Sauvage, 1878, 397.

Hydrophlox Jordan, 1878, 395.

Erogala Jordan \& Brayton, 1878, 395.

Couesius Jordan, 1878, 395.

Chriope Jordan, 1878, 396.

Symmetrurus Jordan, 1878, 396.

Zophendum Jordan, 1878, 396.

Agenigobio Sauvage, 1878, 397.

Heteroleuciscus Sauvage, 1874, 376.

Megagobio Kessler, 1876, 386.

Lythrurus Jordan, 1876, 386.

Erinemus Jordan, 1876, 386.

${ }^{1028}$ This nominal genus is no doubt based on an escaped gold fish. There are no Cyprinide in Australia nor in South America. 
Hexapsephus Günther, 1876, 385.

BRACHYSPONDYLUS ${ }^{\text {102c }}$ von der Marck, 1876.

Aspiorhynchus Kessler, 1879, 400.

Enoplophthalmus Sauvage, 1880, 405.

Paraleuciscus Sauvage, 1880, 406.

Probarbus Sauvage, 1880, 405.

Opsopcodus Hay, 1880, 403.

Semilabeo Peters, 1880, 405.

Mylopharyngodon Peters, 1880, 404.

Distœchodon Peters, 1880, 404.

Paratylognathus Sauvage, 1880, 405.

Stypodon Garman, 1881, 415.

Parachela Steindachner, 1881, 417.

Tirodon Hay, 1882, 419.

Coccotis Jordan, 1882, 419.

Erimystax Jordan, 1882, 419.

Trycherodon Forbes, 1882, 420.

Pachychilon Steindachner, 1882, 421.

Cosmochilus Sauvage, 1882, 421.

Gymnognathus Sauvage, 1884, 429.

Siphateles Cope, 1883, 421.

Tribolodon Sauvage, 1883, 425.

Hypargyrus Forbes, 1884, 428.

Rhodeoides Thominot, 1884, 430.

Oxygeneum Forbes, 1885, 431.

Luxilinus Jordan, 1885, 432.

Hemiculterella Varpakhovskii, 1887, 439.

Miniellus Jordan, 1888, 442.

Platypharndon Herzenstein, 1888, 442.

Chuanchia Herzenstein, 1888, 442.

Acanthonotus (Tickell) Day, 1888, 440.

Metallites Schulze, 1889, 448.

Liparus Schulze, 1889, 448.

Rhynchocypris Günther, 1889, 446.

Parapelecus Günther, 1889, 447.

Scaphiodonichthys Vinciguerra, 1889, 448.

Scombrocypris ${ }^{123}$ Günther, 1889, 446.

Matsya Day, 1889, 445.

Spirlingus Fatio, 1890, 450.

Eucirrichthys Perugia, 1892, 459.

Gymnodiptychus Herzenstein, 1892, 458.

Acanthogobio Herzenstein, 1892, 458.

Pungtungia Herzenstein, 1892, 458.
Oxybarbus Vaillant, 1893, 463.

Aphelichthys Cope, 1893, 460.

Engraulicypris Günther, 1893, 461.

Nematabramis Boulenger, 1894, 464.

Neobola Vinciguerra, 1895, 470.

Evarra Woolman, 1895, 470.

Leucogobio Günther, 1896, 471.

Onychostoma Günther, 1896, 471.

Azteca Jordan \& Evermann, 1896, 473.

Orcella J. \& E., 1896, 473.

Iotichthys J. \& E., 1896, 473.

Opsopœa J. \& E., 1896, 473.

Yuriria J. \& E., 1896, 473.

Apiorhynchus Nikolskii, 1897, 477.

Apiostoma Nikolskii, 1897, 477.

Catlocarpio Boulenger, 1898, 478.

Aztecula Jordan \& Evermann, 1898, 482.

Chelæthiops Boulenger, 1899, 485.

Xystrosus Jordan \& Snyder, 1899, 487.

Falcula J. \& S., 1899, 487.

Xenendum J. \& S., 1899, 487.

Oreoleuciscus Varpachovskii, 1899, 488.

Orcula Jordan \& Evermann, 1900, 489.

Ischikauia J. \& S., 1900, 490.

Otakia J. \& S., 1900, 490.

Leptocypris Boulenger, 1900, 488.

Gyrinochilus Vaillant, 1902, 500.

Culticula Abbott, 1901, 491.

Pterocapoëta Günther, 1902, 498.

Zacco Jordan \& Evermann, 1902, 498.

Paracrossochilus Popta, 1902, 500.

Zezera Jordan \& Fowler, 1903, 505.

Biwia J. \& F., 1903, 505.

Abbottina J. \& F., 1903, 505.

Falcularius Jordan \& Snyder, 1903, 504.

Paracrossocheilus Popta, 1904, 510.

Paranotropis Fowler, 1904, 507.

Luciocyprinus Vaillant, 1904, 511.

Longurio $^{184}$ Jordan \& Starks, 1905, 514.

Fusania J. \& S., 1905, 514.

Coreius J. \& S., 1905, 514.

Neogastromyzon Popta, 1905, 515.

Xenopomichthys Pellegrin, 1905, 515.

Pararhodeus Berg, 1907, 521.

Plagiognathops Berg, 1907, 521.

${ }^{102 \mathrm{c}}$ Paleontogr., 22 : 411 ; or thotype $B$. saropterix Marck. (Omitted in "Genera of Fishes.")

${ }^{103}$ Duplicated under the misprint Scomberopsis in "Genera of Fishes," page 447.

104 The name Longurio, a synonym of Saurogobio, is preoccupied in Diptera. 
Aspiolucius Berg, 1907, 521.

Paraleucogobio Berg, 1907, 521.

Pogonocharax ${ }^{106}$ Regan, 1907, 526.

Pararasbora Regan, 1908, 530.

Mearnsella Seale \& Bean, 1908, 530.

Coccogenia Cocker 11 \& Callaway, 1909, 532.

Candidia Jordan \& Richardson, 1909, 533.

Margariscus Cockerell, 1909, 532.

Macrhybopsis Cockerell \& Allison, 1909, 532.

Temeculina Cockerell, 1909, 532.

Acapoëta Cockerell, 1910, 535.

Rhodeops Fowler, 1910, 535.

Hemigrammocypris Fowler, 1910, 535

Parabarbus Franz, 1910, 535.

Aspiopsis Zugmayer, 1912, 546.

Hesperoleucus Snyder, 1912, 546.

Ageneiogarra Garman, 1912, 543.

Myloleuciscus Garman, 1912, 543.

Acanthorutilus Berg, 1912, 542.

Coripareius Garman, 1912, 543.

Pararutilus Berg, 1912, 542.

Myloleucops Cockerell, 1913, 547.

Leucalburnus Berg, 1914, 552.

Pseudogobiops Berg, 1914, 552.

Chilogobio Berg, 1914, 552.

Schizocypris Regan, 1914, 554.

Tanakia Jordan \& Thompson, 1914, 553.
Chilogobio Berg, 191b, 555.

Lissochilus Weber \& Beaifort, 1916, 561.

Brevigobio Tanaka, 1916, 561.

Brachydanio Weber \& Beaufort, 1916, 561.

Capoëtobrama Berg, 1916, 558.

Acanthalburnus Berg, 1916, 558.

Leucalburnus Berg, 1916, 558.

Leucidius Snyder, 1917, 563.

Sambwa ${ }^{106}$ Annandale, 1918.

Microrasbora ${ }^{10 T}$ Annandale, 1918.

Georgichthys ${ }^{198}$ Nichols, 1918.

Spinibarbus Oshima, 1919, 570.

Phoxiscus Oshima, 1919, 570.

Scaphesthes Oshima, 1919, 570.

Aristichthys Oshima, 1919, 570.

Cultriculus Oshima, 1919, 570.

Formosania Oshima 1919, 570.

Acrossochilus Oshima, 1919, 570.

Raiamas Jordan, 1919, 567.

Extrarius Jordan, 1919, 567.

Lissocheilichthys Oshima, 1920, 572.

Leucisculus Oshima, 1920, 572 .

Rasborinus Oshima, 1920, 572.

Scaphiodontella Oshima, 1920, 572.

Exoglossops ${ }^{180}$ Fowler \& Bean, 1920.

Alisodon ${ }^{200}$ Hay, 1921.

${ }^{105}$ This generic name was based, according to Dr. J. T. Nichols, on an aquarium fish from India wrongly reported from Argentina, a cyprinoid, not a characim. It is a synonym of Esomus Swainson, 1839, and of Nuria Valenciennes, 1842.

${ }^{106}$ Records Ind. Mus., 14: 48; orthotype S. resplendens Annandale.

${ }^{107}$ Records Ind. Mus., 14 : 50 ; logotype M. rubescens Annandale.

${ }^{108}$ Nichols, Proc. Biol. Soc. Wash., $31: 17$; orthotype Georgichthys scaphignathus Nichols.

${ }^{100}$ "Fishes from Soochow, China," Proc. U. S. Nat. Mus., 58: pl. 311; type E. geei Fowler \& Bean.

${ }^{200}$ Proc. U. S. Nat. Mus., 58: 132; orthotype A. mirus Hay.(fossil) ; Pleistocene, Bulverde, Texas.

\section{Family 238. MEDID $\approx$}

Meda Girard, 1856, 269.

Plagopterus Cope, 1874, 375 .

Lepidomeda Cope, 1874, 375.

\section{Family 239. COBITID $\mathbb{E}$}

Cobitis Linnæus, 1758, 14.

Barbatula Linck, 1790, 49.

Misgurnus Lacép ̀̀de, 1803, 65.

\section{(Loaches)}

Nemacheilus Van Hasselt, 1823, 116.

Acanthopsis Van Hasselt, 1823, 116.

Oxygaster Van Hasselt, 1823, 116. 
Acanthophthalmus Van Hasselt, 1823, 116.

Botia Gray, 1831, 138.

Pterozygus De la Pylaie, 1835, 183.

Acanthopsis Agassiz, 1835, 180.

Somileptes Swainson, 1839, 204.

Acoura $^{201}$ Swainson, 1839, 204.

Canthophrys Swainson, 1839, 204.

Diacantha Swainson, 1839, 204.

Psilorhynchus McClelland, 1839, 195.

Schistura McClelland, 1839, 195.

Hymenphysa ${ }^{202}$ McClelland, 1839, 195.

Aperioptus Richardson, 1848, 240.

Cobitichthys Bleeker, 1858, 279.

Syncrossus Blyth, 1860, 294.

Prostheacanthus Blyth, 1860, 294.

Pangio Blyth, 1860, 294.

Apua Blyth, 1860, 294.

Acanthocobitis Peters, 1861, 308.
Paracobitis Bleeker, 1863, 322.

Lepidocephalich thys Bleeker, 1863, 322.

Platacan thus Day, 1865, 367.

Octonema ${ }^{202 a}$ Martens, 1868, 352.

Leptobotia Bleeker, 1870, 355.

Oreias Sauvage, 1874, 376.

Diplophysa Kessler, 1874, 376.

Pseudodon Kessler, 1874, 376.

Paramisgurnus Sauvage, 1878, 397.

Crossostoma Sauvage, 1878, 397.

Octonema Herzenstein, 1887, 438.

Lefua Herzenstein, 1888, 442.

Modigliania Perugia, 1893, 462.

Elxis Jordan \& Fowler, 1903, 505.

Orthrias J. \& F., 1903, 505.

Ussuria Nikolskii, 1904, 510.

Vaillantella Fowler, 1905, 512.

Gobiobotia Kreyenberg, 1911, 540.

Aborichthys Chaudhuri, 1913, 547.

${ }^{201}$ Spelled also Acourus by Swainson.

Usually corrected to Hymenophysa.

${ }^{202 a}$ This name is apparently not preoccupied and is not identical with Lefua.

\section{Family 240. HOMALOPTERID \&E}

Homaloptera Van Hasselt, 1823, 116.

Balitora Gray, 1833, 178.

Helgia Vinciguerra, 1889, 448.

Glaniopsis Boulenger, 1899, 485.

Ellopostoma Vaillant, 1902, 500.

Parhomaloptera Vaillant, 1902, 500.
Gyrinocheilus Vaillant, 1902, 500.

Homalopteroides Fowler, 1905, 513.

Lepturichthys Regan, 1911, 540.

Hemimyzon Regan, 1911, 540.

Blavania Hora, 1922. (See Index.)

\section{Family 241. ADIPOSIID स}

The genus Adiposia, with a well-developed adipose fin, approaches the Nematognathi, and may stand as a separate family pending investigation. Adiposia $^{203}$ Annandale \& Hora, 1921.

${ }^{303}$ "The Fish of Seistan," Records of the Indian Museum, 18: Part IV, pp. 157, 187; type Nemachilus macmahoni Chaudhuri.

\section{Order NEMATOGNATHII}

\section{Family 242. DIPLOMYSTID $\not E$}

Diplomystes Duméril, 1856, 269.

Diplomystes (Duméril) Bleeker, 1862, 311.

Family 243. ARIIDE (Tachysuride) (Sea Cat-fishes)

The name Arius may be provisionally retained in place of Tachysuras, known from a drawing only, perhaps intended for some fresh-water form. 
Tachysurus Lacépède, 1803, 66.

Bagre (Cuvier) Oken, 1817, 100.

Glanis Agassiz, 1829, 131.

Breviceps Swainson, 1838, 194.

Felichthys Swainson, 1839, 203.

Arius Cuvier \& Valenciennes, 1840, 206.

Galeichthys Cuv. \& Val., 1840, 206.

Batrachocephalus Bleeker, 1847, 233.

Osteogeneiosus Bleeker, 1847, 230.

Ketengus Bleeker, 1847, 230.

Ariodes Müller \& Troschel, 1849, 243.

Mystus Gronow, 1854, 259.

Ailurichthys ${ }^{204}$ Baird \& Girard, 1854, 255.

Genidens Castelnau, 1855, 264.

Hexanematichthys Bleeker, 1858, 278.

Selenaspis Bleeker, 1858, 278,

Cephalocassis Bleeker, 1858, 278.

Netuma Bleeker, 1858, 278.

Guiritinga Bleeker, 1858, 278.

Osteogeneiosus Bleeker, 1858, 278.

Batrachocephalus Bleeker, 1858, 278.

Ketengus Bleeker, 1858, 278.

${ }^{204}$ Often written Aelurichthys.

\section{Family 244.}

Cataphractus Catesby, 1771, 32.

Ageneiosus Lacépède, 1803, 66.

Doras Lacépède, 1803, 65.

Ceratorhynchus Agassiz, 1829, 131.

Centrochir Agassiz, 1829, 132.

Auchenipterus ${ }^{205}$ Cuvier \& Valenciennes, 1840.

Trachelyopterus Cuv. \& Val,, 1840, 206.

Euanemus Müller, 1843, 216.

Oxydoras Kner, 1855, 265.

Pseudodoras Bleeker, 1858, 278.

Hemidoras Bleeker, 1858, 278.

Davalla Bleeker, 1858, 278.

Trachycorystes Bleeker, 1858, 279.

Asterophysus Kner, 1858, 282.

Centromochlus Kner, 1858, 282.

Platydoras Bleeker, 1862, 311.

Lithodoras Bleeker, 1862, 311.

Rhinodoras Bleeker, 1862, 311.

Astrodoras Bleeker, 1862, 311.

Pterodoras Bleeker, 1862, 311.

Acanthodoras Bleeker, 1862, 311.

Amblydoras Bleeker, 1862, 311.

Parauchenipterus Bleeker, 1862, 311.
Hemipimelodus Bleeker, 1858, 278.

Ariopsis Gill, 1861, 303.

Parancistrus Bleeker, 1862, 311.

Hemiarius Bleeker, 1862, 311.

Pseudarius Bleeker, 1862, 311.

Catastoma (Kuhl \& Van Hasselt) Bleeker, $1862,312$.

Leptarius Gill, 1863, 324.

Notarius Gill, 1863, 324.

Paradiplomystes Bleeker, 1863, 312.

Rhineastes Cope, 1872, 364.

Astephus Cope, 1873, 368.

Neoarius Castelnau, 1877, 388.

Cathorops Jordan \& Gilbert, 1882, 420.

Nedystoma Ogilby, 1898, 483.

Cinetodus Ogilby, 1898, 483.

Aspistor Jordan \& Evermann, 1898, 482.

Pachyula Ogilby, 1898, 483.

Ancharius Steindachner, 1880, 406.

Nemapteryx Ogilby, 1908, 529 .

Doiichthys Weber, 1913, 551.

Tetranesodon Weber, 1913, 551.

\section{DORADID正}

Trachycorystes Bleeker, 1862, 311.

Pseudauchenipterus Bleeker, 1862, 311.

Auchenipterichthys Bleeker, 1862, 311.

Tetranematichthys Bleeker, 1862, 312 .

Pseudageneiosus Bleeker, 1862, 312.

Trachelyopterichthys Bleeker, 1862, 311.

Dianema Cope, 1872, 363.

Physopyxis Cope, 1872, 363.

Zathorax Cope, 1872, 363.

Glanidium Lütken, 1874, 376.

Wertheimeria Steindachner, 1876, 386.

Epapterus Cope, 1878, 393.

Pseudepapterus Steindachner, 1893.( See Index.)

Leptodoras Boulenger, 1898, 479.

Mormyrostoma Ribeiro, 1912, 545.

Parasturisoma Ribeiro, 1912, 545.

Tatia Ribeiro; 1912, 545.

Tympanopleura Eigenmann, 1912, 542.

Pseudepterus Steindachner, 1915, 558.

Entomocorus Eigenmann, 1917, 562.

Ceratocheilus Ribeiro, 1918, 564.

Tannayia Ribeiro, 1918, 564.

${ }^{200}$ Histoire naturelle des Poissons, 15: 207; orthotype Hypophthalmus nuchalis Spix; later made type of Euanemus. (Omitted in "Genera of Fishes.") 
Family 245. PLOTOSID E

Plotosus Lacépède, 1803, 66.

Tandanus Mitchell, 1839, '196.

Deportator Gistel, 1848, 237.

Paraplotosus $^{208}$ Bleeker, 1862.

Chœroplotosus Kner, 1864, 334.

Copidoglanis $^{205 a}$ Günther, 1864, 332.

Cnidoglanis Günther, 1864, 332.

Gastromyzon Günther, 1874, 375.

Neoplotosus Castelnau, 1875, 378.

Neosilurus Castelnau, 1877, 388.

Eumeda Castelnau, 1878, 393.
Cainosilurus Macleay, 1881, 416.

Endorrhis Ogilby, 1898, 483.

Euristhmus Ogilby, 1899, 487.

Anyperistius Ogilby, 1908, 529.

Porochilus Weber, 1913, 551.

Lambertia Perugia, 1894, 465.

Ostophycephalus ${ }^{207}$ Ogilby, 1899, 487.

Oloplotosus Weber, 1913, 551.

Anodontoglanis Rendahl, 1922 (See Index A).

${ }^{205 a}$ Misprinted Copiodoglanis.

200 "Systema Silurorum Revisum," 100; orthotype Plotosus albilabris Cuvier \& Valenciennes. (Omitted in "Genera of Fishes.")

${ }^{207}$ Based on a starveling example of Cnidoglanis, according to Waite.

\section{Family 246. SILURID IE}

Silurus Linnæus, 1758, 14.

Ompok Lacépède, 1803, 65.

Callichrous Hamilton, 1822, 114.

Wallago Bleeker, 1851, 247.

Glanis Agassiz, 1856, 267.

Belodontichthys Bleeker, 1858, 279.

Pseudosilurus Bleeker, 1858, 279.

Kryptopterus ${ }^{208}$ Bleeker, 1858, 279.

Kryptopterichthys Bleeker, 1858, 279.

Micronema Bleeker, 1858, 279.

\section{(Sheat-fishes)}

Phalacronotus Bleeker, 1858, 279.

Hemisilurus Bleeker, 1858, 279.

Silurichthys Bleeker, 1858, 278.

Silurodes Bleeker, 1860, 293, 312.

Pterocryptis Peters, 1861, 308.

Parasilurus Bleeker, 1862, 310.

Silurodon Kner, 1864, 334, 367.

Neosilurus Steindachner, 1867, 348.

Diastatomycter Vaillant, 1891, 456.

Apodoglanis Fowler, 1905, 512.

${ }^{208}$ Written Cryptopterus by purists.

\section{Family 247. AMEIURIDÆ}

(Horned Pout; Channel Cats)

Bagre Catesby, 1771, 31.

Noturus Rafinesque, 1818, 109.

Pilodictis ${ }^{200}$ Rafinesque, 1819, 110.

Ictalurus ${ }^{210}$ Rafinesque, 1820, 113.

Elliops Rafinesque, 1820, 113.

Leptops Rafinesque, 1820, 112.

Opladelus ${ }^{211}$ Rafinesque, 1820, 112.

Ameiurus $^{212}$ Rafinesque, 1820, 112.

Ilictis Rafinesque, 1820, 112.
Synechoglanis Gill, 1858, 280.

Schilbeodes Bleeker, 1858, 279.

Gronias Cope, 1864, 329.

Rabidus Jordan \& Evermann, 1896, 473.

Haustor J. \& E., 1896, 473.

Villarius Rutter, 1896, 475.

Istlarius Jordan \& Snyder, 1899, 486.

Trogloglanis Eigenmann, 1919, 566.

${ }^{208}$ Mythical, the name corrected to Pelodichthys.

${ }^{210}$ Corrected to Ichthalurus.

${ }^{211}$ Name corrected to Hopladelus.

${ }^{212}$ Also spelled Amiurus. 


\section{Family 248. BAGRID E}

Mystus Gronow, 1763, 21.

Branchiosteus Gill, 1862, 318.

Mystus (Gronow) Scopoli, 1777, 42.

Porcus Isidore Geoffroy St. Hilaire, 1818, 107.

Bucklandium König, 1825, 118.

Bagrus Cuvier \& Valenciennes, 1839, 194.

Olyra McClelland, 1842, 212.

Glyptocephalus Agassiz, 1844, 217.

Bagroides Bleeker, 1851, 248.

Macrones Duméril, 1856, 269.

Clarotes Kner, 1855, 265.

Pseudobagrus Bleeker, 1858, 278.

Melanodactylus Bleeker, 1858, 278.

Chrysichthys Bleeker, 1858, 278.

Octonematichthys Bleeker, 1858, 278.

Rita Bleeker, 1858, 278.

Auchenaspis Bleeker, 1858, 278.

Rama Bleeker, 1858, 279.

Leiocassis Bleeker, 1858, 278.

Bagrichthys Bleeker, 1860, 293, 312.

Pseudobagrus Bleeker, 1860, 293.

Hemibagrus Bleeker, 1862, 312.

Pseudobagrichthys Bleeker, 1862, 312.

Aspidobagrus Bleeker, 1862, 312.

Hypselobagrus Bleeker, 1862, 313

Heterobagrus Bleeker, 1864, 329.

Auchenoglanis Günther, 1864, 334.

Rhinobagrus Bleeker, 1865, 335.

Pelteobagrus Bleeker, 1865, 335.

Gogrius Day, 1867, 344.

Liobagrus Hilgendorf, 1878, 395.

Cranoglanis Peters, 1880, 405.

Neobagrus Bellotti, 1892, 457.

Chrysobagrus Boulenger, 1899, 485.

Gephyroglanis Boulenger, 1899, 485.

Notoglanidium Günther, 1902, 498.

Leptoglanis Boulenger, 1902, 496.

Fluvidraco Jordan \& Fowler, 1903, 505.

FAJUMIA $^{213}$ Stromer, 1904, 511.

Socnopea Stromer, 1904, 511.

Phyllonemus Boulenger, 1906, 517.

Parauchenoglanis Boulenger, 1911, 538.

Liauchenoglanis Boulenger, 1916, 558.

Amarginops Nichols \& Griscom, 1917, 562.

Gnathobagrus Nichols \& Griscom, 1917, 562.

Aoria Jordan, 1919, 567.

Macronoides ${ }^{214}$ Hora, 1921.

Laguvia ${ }^{215}$ Hora, 1921.

${ }^{213}$ Stromer compares this genus to Platystoma, a Brazilian pimelodid. But it probably belongs with Socnopeea to the Bagridce.

${ }^{214}$ Sunder Lal Hora, "Fish and Fisheries of Manipur," Rec. Ind. Mus., 22:

Part III, 179; logotype Batasio affinis Blyth.

216 "Fishes from the Eastern Himalayas," Rec. Ind. Mus., 22: Part V, 740; logotype Pimelodus asperus McClelland.

\section{Family 249. AMBIYCIPITID蚱}

Amblyceps Blyth, 1858, 279.

Akysis Bleeker, 1860, 293.

Acrochordonichthys Bleeker, 1858, 279.

Sosia Vaillant, 1902, 500.

\section{Family 250. SISORID $\mathbb{E}$}

Sisor Hamilton, 1822, 115.

Batasio Blyth, 1860, 294.

Glyptosternon ${ }^{216}$ McClelland, 1842, 212.

Erethistes Müller \& Troschel, 1849, 243.

Bagarius Bleeker, 1853, 253.

Gagata Bleeker, 1858, 279.

Hara Blyth, 1860, 294.

Aglyptosternon Bleeker, 1862, 312.

Euclyptosternum Günther, 1864, 332.

Callomystax Günther, 1864, 332.

Chiloglanis Peters, 1868, 353.

Nanera Day, 1876, 385.

teinia Steindachner, 1881, 417.

:oma Regan, 1905, 515.

${ }^{\text {a1s }}$ Spelled later Glyptosternum. 


\section{Family 251. AMPHILIID无}

Amphilius Günther, 1864, 332.

Chimarrichthys ${ }^{217}$ Sauvage, 1874. Doumea Sauvage, 1879, 401.

Anoplopterus Pfeffer, 1889, 448.

Peltura Perugia, 1892, 459.

Chimarrhoglanis Vaillant, 1897, 478.
Andersonia Boulenger, 1900, 488.

Phractura Boulenger, 1900, 488.

Belonoglanis Boulenger, 1902, 496.

Paraphractura Boulenger, 1902, 496.

Trachyglanis Boulenger, 1902, 496.

Paramphilius Pellegrin, 1907, 526. of Fishes.")

\section{Family 252. LEPIDOGLANID AE}

Said to resemble Chinarrhichthys but to have cycloid scales like a cyprinoid, a character hard to explain.

Lepidoglanis Vaillant, 1889, 448.

\section{Family 253. CHACIDE}

Chaca Gray, 1831, 138.

\section{Family 254. SCHLBEID压}

Schilbe (Cuvier) Oken, 1817, 100.

Ailia Gray, 1831, 138.

Acanthonotus Gray, 1831, 138.

Silonia Swainson, 1839, 203.

Clupisoma Swainson, 1839, 204.

Pachypterus Swainson, 1839, 204.

Pusichthys Swainson, 1839, 204.

Silundia Cuvier \& Valenciennes, 1840, 206.

Eutropius Müller \& Trosche1, 1849, 243.

Schilbeichthys Bleeker, 1858, 279.

Siluranodon Bleeker, 1858, 279.

Helicophagus Bleeker, 1858, 277.
Lais Bleeker, 1860, 293.

Eutropiichthys Bleeker, 1862, 310.

Pseudeutropius Bleeker, 1862, 310.

Gymnallabes Günther, 1867, 345.

Ailichthys Day, 1871, 361.

Anopleutropius Vaillant, 1893, 463.

Parailia Boulenger, 1899, 485.

Physailia Boulenger, 1901, 492.

Pseudolais Vaillant, 1902, 500.

Ansorgia Boulenger, 1912, 542.

Laides Jordan, 1919, 568.

Pareutropius ${ }^{218}$ Regan, 1920.

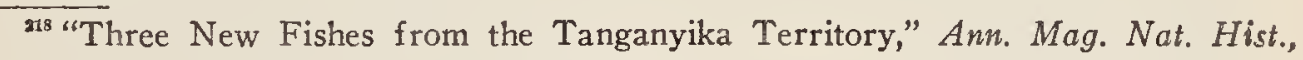
July, 1920, 103; type $P$. micristius Regan.

\section{Family 255. CLARIID无}

Clarias Gronow, 1763, 20.

Clarias (Gronow) Scopoli, 1777, 42.

Macropteronotus Lacépède, 1803, 65.

Heterobranchus Isidore Geoffroy St.

Hilaire, 1818, 107.

Heteropneustes Müller, 1839, 196.

Saccobranchus Cuvier \& Valenciennes, $1840,206$.
Phagorus McClelland, 1844, 220.

Cossyphus McClelland, 1844, 220.

Channalabes Günther, 1873, 369.

Clariallabes Boulenger, 1898, 576.

Allabenchelys Boulenger, 1902, 497.

Dinotopterus Boulenger, 1906, 516.

\section{Family 256. PANGASIID $\mathbb{E}$}

Pangasius Cuvier \& Valenciennes, 1840, Pseudopangasius Bleeker, 1862, 310. 206. 


\section{Family 257. MOCHOKID $\mathbb{E}$}

Synodontis Cuvier, 1817, 100.

Mochokus ${ }^{219}$ Joannis, 1835, 182.

Hemisynodontis Bleeker, 1862, 311.

Pseudosynodontis Bleeker, 1862, 311.

Brachysynodontis Bleeker, 1862, 311.

Leiosynodontis Bleeker, 1862, 311.

Atopochilus Sauvage, 1879, 401.
Oxyglanis Vinciguerra, 1898, 484.

Euchilichthys Boulenger, 1900, 488.

Microsynodontis Boulenger, 1903, 501.

Slatinia Werner, 1906, 521.

Acanthocleithron Nichols \& Griscom, $1917,562$.

${ }^{219}$ Spelled Mochocus by Günther.

\section{Family 258. MALAPTERURID丑 (Torpedinida)}

(Electric Cat-Fishes)

Torpedo o ${ }^{220}$ Forskål, 1771, 32.

Torpedo (Forskål) Gill, 1890.

Malapterurus Lacépède, 1803, 65.

${ }^{220}$ The species examined by Forskal is an electric cat-fish; the synonym is mixed with the electric ray.

\section{Family 259. HELOGENID $\mathbb{E}$}

Helogenes Günther, 1863, 326.

\section{Family 260. HYPOPHTHALMID $巴$}

Hypophthalmus Spix, 1829, 131.

Notophthalmus Hyrtl, 1859, 292.

Davalla Bleeker, 1858, 278.

\section{Family 261. PIMELODID $\mathbb{E}$}

Pimelodus Lacépède, 1803, 65.

Phractocephalus Agassiz, 1829, 131.

Platystoma Agassiz, 1829, 131.

Sorubim Spix, 1829, 132.

Pirarara Spix, 1829, 173.

Pteronotus Swainson, 1839, 204.

Calophysus Müller, 1843, 216.

Abron Gistel, 1848, 237.

Sciades Müller \& Troschel, 1849, 243.

Conostome $e^{221}$ Duméril, 1856, 269.

Conorhynchos Bleeker, 1858, 278.

Zungaro Bleeker, 1858, 278.

Rhamdia Bleeker, 1858, 278.

Pinirampus Bleeker, 1858, 278.

Sciadeichthys Bleeker, 1858, 278.

Heptapterus Bleeker, 1858, 278.

Piratinga Bleeker, 1858, 279.

Platynematichthys Bleeker, 1858, 279.

Piramutana Bleeker, 1858, 279.

Batrachoglanis Gill, 1858, 281.

Pimelonotus Gill, 1858, 281.

${ }^{221}$ In French form only.
Pseudohypophthalmus Bleeker, 1862, 312.

Pimelotropis Gill, 1859, 291.

Brachyplatystoma Bleeker, 1862, 312.

Hemisorubim Bleeker, 1862, 312.

Pseudoplatystoma Bleeker, 1862, 312.

Hemiplatystoma Bleeker, 1862, 312.

Sorubimichthys Bleeker, 1862, 312.

Platystomatichthys Bleeker, 1862, 312.

Leiarius Bleeker, 1862, 312 .

Pseudariodes Bleeker, 1862, 312.

Malacobagrus Bleeker, 1862, 312.

Parabagrus Bleeker, 1862, 312.

Pseudorhamdia Bleeker, 1862, 310.

Pseudopimelodus Bleeker, 1863, 322.

Notoglanis Günther, 1864, 332.

Bagropsis Lütken, 1874, 376.

Pimelodina Steindachner, 1876, 387.

Lophiosilurus Steindachner, 1876, 387.

Nannoglanis Boulenger, 1887, 437.

Rhamdella Eigenmann \& Eigenmann, $1888,440$.

Luciopimelodus E. \& E., 1888, 440. 
Pimelodella E. \& E., 1888, 440.

Duopalatinus E. \& E., 1888, 440.

Sciadeoides E. \& E., 1888, 440.

Steindachneria E. \& E., 1888, 440.

Nemuroglanis E. \& E., 1889, 445.

Acentronichthys E. \& E., 1889, 445.

Phrenatobius Goeldi, 1898, 480.

Paulicea Ihering, 1898, 480.

Perugia Eigenmann \& Norris, 1900, 489.

Iheringichthys E. \& N., 1900, 489.

Bergiella E. \&. N., 1900, 489.

Gœldiella E. \& N., 1900, 489.

Imparfinis E. \& N., 1900, 489.

Bergiaria E. \& N., 1901, 493.

Typhlobagrus Ribeiro, 1907, 526.

Rhamdioglanis Ihering, 1907, 524.

Tænionema Eigenmann \& Bean, 1907, 522.

Zungaropsis Steindachner, 1908, 531.

Megalonema Eigenmann, 1910, 535, 542.

Cephalosilurus Haseman, 1911, 539.
Platysilurus Haseman, 1911, 539.

Rhamdiopsis Haseman, 1911, 539.

Microglanis Eigenmann, 1912, 542.

Brachy glanis Eigenmann, 1912, 542.

Leptoglanis Eigenmann, 1912, 542.

Myoglanis Eigenmann, 1912, 542.

Chasmocranus Eigenmann, 1912, 542.

Nannorhamdia Regan, 1913, 550.

Cetopsorhamdia Eigenmann, 1916, 558.

Cheirocerus Eigenmann, 1917, 562.

Leptorhamdia Eigenmann, 1918.

Typhlobagrus Ribeiro, 1918, 564.

Steindachneridion Eigenmann \& Eigenmann, 1919, 566.

Laimumena Sauvage, 1884, 430.

Platy pogon Starks, 1913, 551.

Breviglanis ${ }^{222}$ Eigenmann, 1909.

Chasmocephalus $^{223}$ Eigenmann, 1909.

222 "Fresh Water Fish S. A.," 384; type B. frenata Eigenmann.

${ }^{223}$ "Catal. and Bibliogr. S. Am. Fresh Water Fishes," 1909, 384; type Ch. longior, Eigenmann. (Omitted in "Genera of Fishes.")

\section{Family 262. BUNOCEPHALIDE}

Bunocephalus Kner, 1855, 265.

Bunocephalichthys Bleeker, 1858, 279.

\section{Family 263.}

Aspredo Ginnow, 1763, 20.

Aspredo (Gronow) Scopoli, 1777, 42.

Platystacus Klein, 1779, 168.

Platystacus (Klein) Bloch, 1794, 53.

Platistus Rafinesque, 1815, 171.

Family 264

Eremophilus Humboldt, 1806, 76.

Thrycomycterus ${ }^{224}$ Humboldt \& Valenciennes, 1811, 170.

Pygidium Meyen, 1835, 182.

Trichomycterus Valenciennes, 1846, 230.

Vandellia Cuvier \& Valenciennes, 1846 , 407.

Nematogenys Girard, 1854, 258.

Pareiodon ${ }^{228}$ Kner, 1855, 265.

Stegophilus Reinhardt, 1858, 284.

${ }^{224}$ Later spelled Trichomycterus.

${ }^{225}$ Later spelled Pariodon.
Dysichthys Cope, 1874, 374.

Xiliphius Eigenmann, 1912, 543.

\section{ASPREDINID丑}

Cotylephorus Swainson, 1839, 204.

Aspredinichthys Bleeker, 1858, 279.

Agmus Eigenmann, 1912, 542.

Chamaigenes Eigenmann, 1912, 542.

\section{PYGIDIID $\approx$}

Astemomycterus Guichenot, 1860, 296.

Trachypoma Giebel, 1870, 357.

Pariolius Cope, 1872, 363.

Tridens Eigenmann \& Eigenmann, 1889, 446.

Pseudostegophilus E. \& E., 1889, 446.

Miuroglanis E. \& E., 1889, 446.

Acanthopoma Lütken, 1891, 455.

Henonemus Eigenmann \& Ward, 1907, 522. 
Homodiætus E. \& W., 1907, 522.

Hatcheria Eigenmann, 1909, 533.

Ochmacanthus Eigenmann, 1912, 542.

Paravandellia Ribeiro, 1912, 545.

Gyrinurus Ribeiro, 1912, 545.
Cobitiglanis Fowler, 1914, 553.

Pleurophysus Ribeiro, 1918, 564.

Urinophilus Eigenmann, 1918, 563.

Branchoica Eigenmann, 1918, 563.

Scleronema Eigenmann, 1918, 563.
Family 265.

Cetopsis Agassiz, 1829, 131.

Hemicetopsis Bleeker, 1862, 311.

Pseudocetopsis Bleeker, 1862, 311.

Paracetopsis (Guichenot') Bleeker, 1862, 312.

Rept. Princeton Univ. Exp. Patagonia, 111-398, 1910; type Cetopsis occidentals Steindachner. (Omitted in "Zoological Record.") Same as Paracetopsis Eigenmann \& Bean, preoccupied.

$$
\text { - Family } 266 .
$$

Astroblepus Humboldt, 1806, 76.

Cyclopium Swainson, 1839, 204.

Arges Cuvier \& Valenciennes, 1840, 206.

\section{Family 267. CALLICHTHYID $\mathbb{E}$}

Callichthys Gronow, 1763, 21.

Callichthys (Gronow) Scopoli, 1777, 42.

Cataphractus Bloch, 1793, 51.

Corydoras Lacépède, 1803,66 .

Cordorinus Rafinesque, 1815, 91.

Hoplisoma Swainson, 1839, 203.

Hoplosternum Gill, 1858, 281.

Scleromystax Günther, 1864, 332.
Brochis Cope, 1872, 363.

Chrenothorax Cope, 1878, 394.

Decapogon ${ }^{228}$ Eigenmann \& Eigenmann, $1888,440$.

Osteogaster Cope, 1894, 464.

Aspidoras thering 1907, 524.

Cascadura Ellis, 1913, 547.

Cataphractops Fowler, 1915, 556.

${ }^{226 a}$ Decapogon Ribeiro, p. 565, "Genera of Fishes," should be canceled.

\section{Family 268. LORICARIID $\mathbb{E}$}

Loricaria Linnæus, 1758, 14.

Plecostomus Gronow, 1763, 21.

Hypostomus Lacépède, 1803, 66.

Acanthicus Spix, 1829, 131.

Rhinelepis Spix, 1829, 131.

Sturisoma Swainson, 1839, 203.

Ancistrus Kner, 1854, 260.

Acestra Kner, 1854, 260.

Hemiodon Kner, 1854, 260.

Acanthodemus Castelnau, 1855, 264.

Pterygoplichthys Gill, 1858, 281.

Hemiancistrus Bleeker, 1862, 311.

Pseudancistrus Bleeker, 1862, 311.

Plecostomus (Gronow) Bleeker, 1862.

Pseudorinelepis Bleeker, 1862, 311.

Hemiloricaria Bleeker, 1862, 311.

Pseudacanthicus Bleeker, 1862, 311.
Parahemiodon Bleeker, 1862, 311.

Pseudoloricaria Bleeker, 1862, 311.

Loricariichthys Bleeker, 1862, 311.

Oxyloricaria Bleeker, 1862, 311.

Rineloricaria Bleeker, 1862, 311.

Pseudohemiodon Bleeker, 1862, 311.

Hemiodontich thys Bleeker, 1862, 311.

Cochliodon Heckel, 1864, 332.

Hypoptopoma Günther, 1868, 351.

Liposarcus Günther, 1864, 332.

Otocinclus Cope, 1872, 363.

Xenomystus Lütken, 1873, 370.

Harttia Steindachner, 1876, 387.

Hassar Eigenmann \& Eigenmann, 1888, 440.

Neoplecostomus E. \& E., 1888, 440.

Farlowella E. \& E., 1889, 445. 
Oxyropsis E. \& E., 1889; 445.

Hisonotus E. \& E., 1889, 445.

Parotocinclus E. \& E., 1889, 445.

Panaque E. \& E., 1889, 446.

Delturus E. \& E., 1889, 446.

Hemipsilichthys E. \& E., 1889, 446.

Microlepidogaster E. \& E., 1889, 445.

Aristommata Holmberg, 1893, 462.

Xenocara Regan, 1904, 510.

Reganella ${ }^{227}$ Eigenmann, 1905.

Thysanocara ${ }^{228}$ Regan, 1906, 520.

Pogonopoma Regan, 1907, 526.

Kronichthys Ribeiro, 1908, 530.
Corymbophanes Eigenmann, 1909, 532.

Lithogenes Eigenmann, 1909, 532.

Canthopomus $^{220}$ Eigenmann, 1909.

Acestridium Haseman, 1911, 539.

Lithoxus Eigenmann, 1912, 542.

Stoniella Fowler, 1914, 553.

Diapaletoplites Fowler, 1915, 556.

Leptoancistrus Meek \& Hildebrand, 1916, 559.

Pareiorhaphis ${ }^{230}$ Ribeiro, 1918.

Pseudotocinclus Nichols, 1919, 569.

Cheirododus $^{231}$ Eigenmann, 1921.

${ }^{227}$ Eigenmann, Science, 21: 793, May 18, 1905; orthotype Hemiodon depressus

Kner; substitute for Hemiodon; preoccupied in mollusks.

${ }^{229}$ Type Ancistrus cirrhosa Kner.

${ }^{220}$ Cat. Fresh Water Fishes S. A. 407; type Rhinelepis genibarbis Cuv. \& Val. (Omitted in "Genera of Fishes.")

${ }^{230}$ Rev. Soc. Sci. Rio de Janeiro, 2: 106; orthotype Hemipsilichthys calmoni Ribeiro.

${ }^{251}$ Orthotype Plecostomus hondre Regan.

\section{Order INIOMI}

The fishes of this order, mostly inhabitants of depths of the sea and thus more or less degenerate structurally, bear a strong external resemblance to the stomiatoid Isospondyli, with which they have been usually associated and with which they agree in possessing an adipose dorsal fin, as in the Salmonidce, Characinida, Silurida, and Percopsida. The persistence of this primitive character does not necessarily indicate close relationship among the fishes possessing it. In the Iniomi, the premaxillary is well developed, excluding the maxillary from the margin of the upper jaw.

\section{Family 269. CHEIROTHRICID在}

MEGAPUS Marck \& Schlüter, 1865, 339.

Cheirothrix ${ }^{232}$ Pictet \& Humbert, 1866 , 342.

${ }^{232}$ Spelled Chirothrix by authors.

\section{Family 270.}

Aulopus Cuvier, 1817, 98.

SARDinioides von der Marck, 1858, 283.
Exoccetordes Davis, 1885, 431.

Megristopus Landois, 1894, 465.

\section{Family 271. SYNODONTID正}

\section{(Lizard Fishes)}

Synodus Gronow, 1763, 20.

Saurus Catesby, 1771, 29.

Synodus (Gronow) Scopoli, 1777, 42.

Soarus ${ }^{23}$ Linck, 1790, 49.

\footnotetext{
${ }^{238} \mathrm{~A}$ misprint for Saurus, the genus undescribed and unidentifiable.
} 
Tirus Rafinesque, 1810, 80.

Saurus Cuvier, 1817, 98.

Alpismaris Risso, 1826, 120.

Laurida Swainson, 1839, 203.

Triurus Swainson, 1839, 203.

Saurida Cuvier \& Valenciennes, 1849, 242.

Sauridichthys Bleeker, 1858, 278.
Trachinocephalus Gill, 1861, 303 .

Bathysaurus Günther, 1878, 395.

Pelopsia Facciolà, 1883, 423.

Bathylaco Goode \& Bean, 1895, 467.

Goodella Ogilby, 1897, 477.

Xystodus Ogilby, 1910, 536.

\section{Family 272. HARPODONTID}

(Bombay Ducks)

Harpodon Le Sueur, 1825, 172.

\section{Family 273. CHLOROPHTHALMID $\approx$}

Chlorophthalmus Bonaparte, 1840, 142, 206.

${ }^{24}$ Proc. U. S. Nat. Mus., 111: 183; type H. chalybeius Goode. (Omitted in "Genera of Fishes.")

\section{Family 274. PARALEPID王 (Sudide)}

Sudis Rafinesque, 1810, 80.

Paralepis Cuvier, 1817, 104.

Prymnothonus Richardson, 1844, 223.

Arctozenus Gill, 1864, 330.

ANAPTERUs Sauvage, $1873,371$.

Symproptopterus Cocco, 1885, 430.

Lestidium Gilbert, 1905, 513.
Hyphalonedrus ${ }^{234}$ Goode, 1880.

Bathysauropsis Regan, 1911, 540.

2256 "Fish Fauna Cal. Tertiary," 250; orthotype Trossulus exoletus Jordan.

${ }^{236}$ Loc. cit., 250; orthotype Lestichthys porteousi Jordan.

Family 275. EVERMANELLIDE (Odontostomida; Scopelarchida)

The members of the small group are not all well known, and may prove incongruous.

Odontostomus Cocco, 1838, 191.

Neosudis Castelnau, 1873, 368.

Scopelarchus Alcock, 1896, 470.
Notolepis Dollo, $1908,527$.

Parasudis Regan, 1911, 540.

Lestidiops Hubbs, 1916, 559.

Notosudis Waite, 1916, 561.

Trossulus $^{225}$ Jordan, 1921.

LESTICHтHYs ${ }^{298}$ Jordan, 1921.

\section{Family 276. BATHYPTEROIDE}

Bathypterois Günther, 1878, 395.

Synapteretmus $^{277}$ Goode \& Bean, 1895.
Evermane1la Fowler, 1901, 493.

Dissomma Brauer, 1902, 497.

Promacheon Weber, 1913, 551.

237 "Oceanic Ichthyology," 64; orthotype Bathypterois quadrifilis Günther. (Omitted in "Genera of Fishes.")

\section{Family 277. BENTHOSAURID压}

Benthosaurus Goode \& Bean, 1886, 435. 


\section{Family 278. MYCTOPHIDE (Scopelide)}

\section{(Lantern Fishes)}

Of the numerous fossil genera mentioned below, several may belong to related families, rather than to Myctophida.

Myctophum Rafinesque, 1810, 82.

Scopelus Cuvier, 1817, 98.

Macrostoma Risso, 1826, 120.

Nyctophus Cocco, 1829, 133.

Alysia Lowe, 1839, 195.

Lampanyctus Bonaparte, 1840, 142, 206.

Rhinellus Agassiz, 1844, 217.

Osmeroldes Agassiz, 1844, 219.

Holosteus Agassiz, 1844, 219.

Acrognathus Agassiz, 1844, 219.

Phanerobranchus Cocco, 1846, 574.

Cyrtorhynchus Costa, 1855, 574 .

Rhamphornimia Costa, 1857, 275.

Sardinius von der Marck, 1858, 283.

Palimphemus Kner, 1862, 319.

Neoscopelus Johnson, 1863, 575.

LEPTosomus von der Marck, 1863, 328.

Microcerlia von der Marck, 1863, 328.

TACH YN ECTES von der Marck, 1863, 328.

Dasyscopelus Günther, 1864, 333.

Notoscopelus Günther, 1864, 333.

BRACH YSPONDYLUS Marck \& Schlüter, $1865,339$.

Dermatoptychus M. \& S., 1865, 339.

DACTYLopogon M. \& S., $1865,339$.

OPISTH OPTERY X ${ }^{228}$ Pictet \& Humbert, $1866,342$.
Hemisaurida Kner, 1867, 346.

Tydeus Sauvage, 1870,359 .

Parascopelus Sauvage, 1873, 371.

Gymnoscopelus Günther, 1873, 369.

Neoscopelus Castelnau, 1875, 378 .

ICHTHYoTRINGA Cope, 1878, 394.

SCopeloides Wettstein, 1886; 436.

Nannobrachium Günther, 1887, 438.

Omionon Bassani, 1888, 440.

Scopelengys ${ }^{239}$ Alcock, 1890, 449.

Stenobrachius Eigenmann \& Eigenmann, $1890,450$.

Catablemella E. \& E., 1890, 450.

Diaphus E. \& E., 1891, 454.

Tarletonbeania E. \& E., 1891, 454.

Rhinoscopelus Lütken, 1892, 458.

Benthosema Goode \& Bean, 1895, 467.

Collettia G. \& B., 1895, 467.

Lampadena G. \& B., 1895, 467.

Aëthoprora G. \& B., 1895, 467.

Electrona $^{2+0}$ G. \& B., 1895, 467.

Nematonotus Woodward, 1899, 488.

Centrobranchus Fowler, 1904, 507.

Scopelopsis ${ }^{241}$ Brauer, 1906.

Protoblepharon Steche, 1909, 534.

Nyctimaster ${ }^{242}$ Jordan, 1922.

\footnotetext{
${ }^{238}$ Misprinted Opistopteryx.

${ }^{239}$ Misprinted Scopelogenys in Zoological Record and in "Genera of Fishes."

240 "Oceanic Ichthyology," 91; orthotype Scopelus rissoi Cocco. (Omitted in "Genera of Fishes.")

211 "Die Tiefseéfische," 146; type S. multipunctatus Brauer. (Omitted in "Genera of Fishes.")

${ }^{212}$ Proc. U. S. Nat. Mus., 645, 1921 ; orthotype Lampanyctus jordani Gilbert.
}

\section{Family 279. IPNOPID $\nexists$}

Ipnops Günther, 1878, 395.

Family 280. OMOSUDID蚱

Omosudis Günther, 1887, 438.

\section{Family 281. MALACOSTEID㤅}

Malacosteus Ayres, 1849, 240.

Bathylychnus Brauer, 1902, 497. 


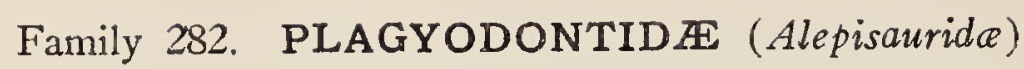 \\ (Hand-saw Fishes)}

Plagyodus ${ }^{2+3}$ (Steller) Pallas, 1811, 84.

Alepisaurus Lowe, 1833, 179.

Istieus Agassiz, 1844, 219.

Caulopus Gill, 1862, 314.
Plagyodus (Steller) Günther, 1867, 345.

Apateodus Woodward, 1901, 496.

Eugnathosaurus Regan, 1913, 550.

${ }^{248} \mathrm{This}$ name has been questioned as appearing in an oblique case only, as "Plagyodontem."

\section{Suborder CETUNCULI}

(New suborder)

The two families which follow are quite distinct from the other Iniomi, doubtless forming a separate suborder among the deep-sea fishes.

\section{Family 283. RONDELETIIDA}

Rondeletia Goode \& Bean, 1894, 465.

\section{Family 284. CETOMIMIDEE}

Cetomimus Goode \& Bean, 1894, 465. Cetostoma Zugmayer, 1914, 555.
Pelecinomimus Gilchrist, 1922. (See Index.)

\section{Suborder CHONDRICHTHYES}

A curious group of very uncertain relationships.

\section{Family 285. ATELEOPODID $\approx$}

Ateleopus Temminck \& Schlegel, 1846, 230.

Podateles Boulenger, 1902, 497.
Ijimaia Sauter, 1905, 516.

Parateleopus Smith \& Radcliffe, 1912, 544.

\section{Order XENOMI}

\section{Family 286. DALLIIDE}

Dallia Bean, 1879, 398.

(Alaska Black-fish)

\section{Order HAPLOMI}

The Haplomi are nearly related to the Isospondyli, although lacking the mesocoracoids characteristic of the latter order and of the Ostariophysi. Externally they have much in common with the Microcyprini or Cyprinodontes, with which most writers have united them. The maxillary, however, contintes, as in the Isospondyli, to form the side of the upper jaw, but in the Haplomi, as in all the families which follow in this linear series, it ceases to bear teeth, and is gradually relegated to a subordinate position. It would appear therefore that the Haplomi and Cyprinodontes are actually rather closely related, though definable as separate orders or suborders. The Xenomi are doubtless near the Haplomi, but are quite as distinct from the latter as the Cyprinodontes. 
The following remarks of Jordan \& Evermann ("Fish. N. M. Am.," I, 623) are still pertinent: "While our knowledge of the osteology and embryology of most of the families of fishes is very incomplete, it is evident that the relationships of the groups can not be shown in any linear series, or by any conceivable arrangement of orders and suborders. The living teleost fishes have sprung from many lines of descent, their relationships are extremely diverse, and their differences are of every possible degree of value. The ordinary schemes have magnified the importance of a few common characters, at the same time neglecting other diferences of equal importance. No system of arrangement which throws these fishes into large groups can ever be definite or permanent."

\section{Family 287. CROSSOGNATHID $\mathbb{E}$}

Crossognathus Pictet \& Loriol, 1859, 292.
Apsopelix Cope, 1871, 360.

Pelycorapis ${ }^{244}$ Cope, $1874,375$.

${ }^{24}$ Misprinted Pelecorapis.

\section{Family 288. ESOCID五 (Luciide)}

(Pikes)

Esox ${ }^{245}$ Linnæus, 1758, 14.

Lucius Geoffroy, 1767, 24.

Lucius Klein, 1775, 40.
Picorellus ${ }^{246}$ Rafinesque, $1820,112$.

Mascalongus Jordan, 1878, 396.

Kenoza Jordan \& Evermann, 1896, 473.

Lucius Rafinesque, $1810,80$.

${ }^{245}$ This use of Esox follows the decision of the International Commission of Zoological Nomenclature. The name has been also employed in place of Belone.

${ }^{240}$ A mythical pickerel.

Family 289. UMBRID $\mathbb{E}$

(Mud Minnows)

Umbra (Kramer) Gronow, 1763, 20.

Melanura Agassiz, 1853, 252.

Umbra (Gronow) Scopoli, 1777, 42.

\section{Order CYPRINODONTES}

\section{(Microcyprini)}

The Microcyprini or Cyprinodontes have been usually placed in one or two families. It seems to us that more should be recognized. These fishes bear considerable resemblance to the Haplomi, with which group they have usually been united. But the structure of the mouth is quite different, the premaxillary forming the whole side of the upper jaw, the toothless maxillary lying above it as a sort of secondary bone, as in all the percoid fishes. The posterior insertion of the dorsal fin is characteristic of both groups as, well as of most of the Synentognathi, far from which they should not be placed in a natural system. 
Hitherto the viviparous and ovipafous Cyprinodonts have been placed in a single family, but the differences within the group are extensive and if the species were not all of small size they would certainly be assigned to two or more family groups. As here understood, the oviparous species, with the anal fin not modified in the male, constitute the family of Cyprinodontida.

\section{Family 290. CYPRINODONTID死 (Killifishes)}

The Funduline, with pointed teeth, and the Cyprinodontince, with the teeth incisor-like, form a natural family widely diffused in the North Temperate Zone.

Fundulus Lacépède, 1803, 65.

Hydrargira ${ }^{247}$ Lacépède, 1803, 67.

Cyprinodon Lacépède, 1803, 68.

Prinodon Rafinesque, 1815, 90.

Lebia $^{248}$ (Cuvier) Oken, 1817, 99.

Aphanius Nardo, 1827, 121.

Aplocheilus ${ }^{249}$ McClelland, 1839, 195.

Panchax Cuvier \& Valenciennes, 1846, 407.

Encrotes Gistel, 1848, 236.

Zygonectes Agassiz, 1853, 252.

Tellia Gervais, 1853, 253.

Fundulichthys Bleeker, 1859, 288.

Adinia Girard, 1859, 290.

Lucania Girard, 1859, 290.

Micromugil Gulia, 1861, 307.

Rivulus Poey, 1860, 299.

Aplocheilichthys Bleeker, 1861, 321.

Lebistes $^{250}$ de Filippi, 1862.

Micristius Gill, 1865, 337.

Lycocyprinus Peters, 1868, 353.

Nothobranchius Peters, 1868, 353.
Problebias Sauvage, 1874, 376.

Pachystetus (Aymard) Sauvage, 1874, 376.

Cynolebias Steindachner, 1876, 387.

Xenisma Jordan, 1877, 390.

Jordanella Goode \& Bean, 1879, 399.

Borborys (Broussonet) G. \& B., 1885, 432.

Geph yrura Cope, 1891, 454.

Proballostomus Cope, 1891, 454.

Neolebias Steindachner, 1894, 465.

Pterolebias Garman, 1895, 467.

Fontinus Jordan \& Evermann, 1896, 473.

Pachylebias Woodward, 1901, 496.

Procatopus Boulenger, 1904, 506.

Oryzias Jordan \& Snyder, 1906, 519.

Mohanga Boulenger, 1911, 538.

Lamprichthys Regan, 1911, 540.

Galasaccus Fowler, 1916, 559.

Chriopeops Fowler, 1916, 559.

Oxyzygonectes Fowler, 1916, 559.

Parafundulus Eastman, 1917, 561.

${ }^{247}$ Usually corrected to Hydrargyra.

${ }^{248}$ Later spelled Lebias.

${ }^{240}$ Later corrected to Haplochilus.

${ }^{250}$ Archiv. Zool. Anat., etc., 1: 69; orthotype L. pœciloides de Filippi. (Omitted in "Genera of Fishes.")

\section{Family 291. ORESTIID $\mathbb{E}$}

This little group comprises allies of Fundulus, which have the lower pharyngeals enlarged and provided with molar teeth. The ventral fins are wanting. The family, probably once more widely diffused, is now confined to Lake Titicaca and to the desert streams about Death Valley, Nevada.

Orestias Cuvier \& Valenciennes, 1846, 228. Empetrichthys Gilbert, 1893, 461. 


\section{Family 292. CHARACODONTIDAE}

This family and the next include those viviparous Cyprinodonts in which the first rays of the male are somewhat modified but not transformed into an intromittent organ.

Characodon Günther, 1866, 340.

Zoogoneticus Meek, 1902, 500.

\section{Family 293. GOODEID瓜}

Girardinichthys Bleeker, 1860, 294. Limnurgus Günther, 1866, 340. Goodea Jordan, 1879, 400.
Xenendum Jordan \& Snyder, 1899, 487.

Skiffia Meek, 1902, 500.

Chapalichthys Meek, 1902, 500.

\section{Family 294. PECILID $\mathbb{E}$}

(Top Minnows)

This family includes provisionally those viviparous Cyprinodonts having the anal fin modified into an intromittent organ. All the Pceiliidee are confined to warmer parts of America. It is possible that the family should be further subdivided. The Anablepince with "four eyes" (the eye crossed by a partition) and the Gambusince with teeth as in the Funduline, may well be detached on account of differences in dentition. In the Pceciliince the teeth are slender and movable, while in the Gambusiince they are conic and fixed as in the Funduline. The name Paciliide is probably sufficiently distinct from Pocilide, a family of beetles.

Pæcilia Bloch \& Schneider, 1801, 59.

Mollienesia Le Sueur, 1821, 114.

Alazon Gistel, 1848, 237.

Xiphophorus Heckel, 1848, 238.

Heterancria Agassiz, 1853, 251.

Pcecilops Pomel, 1853, 254.

Limia Poey, 1854, 261.

Gambusia Poey, 1854, 260.

Girardinus ${ }^{251}$ Poey, 1854, 261.

Belonesox Kner, 1859, 292, 298.

Pseudoxiphophorus Bleeker, 1859, 287.

Hemixiphophorus Bleeker, 1859, 288.

Trifarcius Poey, 1860, 299.

Limia Poey, 1861, 308.

Pœcilodes Steindachner, 1863, 328.

Platypœcilus Günther, 1866, 341 .

Acropœcilia Hilgendorf, 1889, 447.

Glaridodon Garman, 1895, 467.

Cnestrodon Garman, 1895, 467.

Gambusinus Jordan \& Evermann, 1896, 473.

Glaridichthys Garman, 1896, 471.

Toxus Eigenmann, 1903, 501.

Cynodonichthys Meek, 1904, 510.
Paragambusia Meek, 1904, 510.

Acanthophacelus Eigenmann, 1907, 522, 533.

Ilyodon Eigenmann, 1907, 522.

Phalloptychus Eigenmann, 1907, 522, 559.

Phalloceros Eigenmann, 1907, 522.

Petalosoma ${ }^{252}$ Regan, 1908, 530.

Tomeurus Eigenmann, 1909, 533.

Alfaro Meek, 1912, 544.

Cynopœcilus Regan, 1912, 545.

Petalurichthys Regan, 1912, 545.

Gulapinnus Langer, 1913, 549.

Priapichthys Regan, 1913, 550.

Pseudopœcilia Regan, 1913, 550.

Priapella Regan, 1913,550.

Pocilopsis Regan, 1913, 550.

Brachyrhaphis Regan, 1913, 550.

Leptorhaphis Regan, 1913, 550.

Pamphoria Regan, 1913, 550.

Pamphorichthys Regan, 1913, 550.

Heterophallus Regan, 1914, 555.

Diphyacantha Henn, 1916, 559.

Phallatorhynus Henn, 1916, 559.

Neoheterandria Henn, 1916, 559.

${ }^{251}$ According to Garman this genus is distinct from Heterandria.

${ }^{202}$ Misprinted Palosoma in Dean's "Bibliography of Fishes." 


\section{Family 295. FITZROYIID I}

Fitzroyia Günther, 1866, 340.

Jenynsia Günther, 1866, 341.

\section{Fanily 296. ANABLEPID $\approx$}

\section{(Quatro Ojos)}

Arableps Gronow, 1763, 20.

Anableps (Gronow) Scopoli, 1777, 42.
Anableps (Gronow) Bloch \& Schneider, 1801, 59.

\section{Family 297. PHALLOSTETHID EE}

Phallostethus Regan, 1913, 550.

Neostethus Regan, 1916, 560.

\section{Family 298. AMBLYOPSID五}

(Cave Blind Fishes)

Amblyopsis Dekay, 1842, 210.

Chologaster Agassiz, 1853,251.
Typhlichthys Girard, 1859, 290.

Troglichthys Eigenmann, 1899, 485.

\section{Family 299. ADRIANICHTHYID臣}

Adrianichthys Weber, 1913, 552.

Xenopœcilus Regan, 1911, 540.

\section{Order SYNENTOGNATHI}

\section{Family 300 . FORFICID王}

Forfex Jordan, 1919, 568.

ZELOTES $^{259}$ Jordan, 1921.

${ }^{253}$ "Fossil Fish. S. Cal.," 36; orthotype Z. alhambrce Jordan.

\section{Family 300A. ROGENIID E}

Rogenio Jordan, 1907, 525.

\section{Family 301. XENESTHIDE}

The genus Xenesthes is based on a long and strong premaxillary bone, seven inches in length, armed anteriorly with a row of large teeth. The toothless maxillary is apparently united with this bone. As the Ichthyodectida have the premazillary short, forming the front only of the edge of the upper jaw, Xenesthes can not be allied to them. It is perhaps near the Belonida, although the type specimen shows none of the small teeth characteristic of the latter, and the snout could not have been attenuate. It bears also a certain resemblance to Plagyodus, but in the latter the bones are soft and smooth, while in Xenesthes they are rigid and rough. XeNesthes Jordan, 1907, 524.

\section{Family 302. BELONID㤅}

(Hound Fishes; Needle Fishes)

Mastacembelus Klein, 1775, 40.

Raphistoma ${ }^{254}$ Rafinesque, 1815, 91.

Belone Cuvier, 1817, 99.

Strongylura ${ }^{254 a}$ Van Hasselt, 1824.
Tylosurus Cocco, 1833, 178.

Ramphistoma Swainson, 1839, 203.

Potamorrhaphis Günther, 1866, 340.

Athlennes Jordan \& Fordice, 1886, 435.

${ }^{254}$ Written Ramphistoma by authors.

${ }^{254 a}$ Bull. Sci. Nat. Ferussac, 2: 374; orthotype Strongylura caudimaculata Van Hasselt $=$ Belone strongylura Van Hasselt, 1823. (Omitted in "Genera of Fishes.") 
Ablennes J. \& F., 1886, 435.

Eurycaulus Ogilby, 1908, 529.

Stenocaulus Ogilby, 1908, 529.

Xenentodon Regan, 1911, 540.
Petalichthys ${ }^{255}$ Regan, 1911.

Platybelone ${ }^{255 a}$ Fowler, 1919.

Tropidocaulus Ogilby, 1919, 569.

Beltion Jordan, 1920, 571.

${ }^{235}$ Ann. Mag. Nat. Hist., 332; orthotype P. capensis Regan. (Omitted in "Genera of Fishes.")

${ }^{255 a}$ Proc. Acad. Nat. Sci. Phila., 71:2; orthotype Belone platyura Bennett (same as Tropidocaulus of slightly later date).

\section{Family 303. SCOMBERESOCID $\mathbb{E}$}

(Sauries)

Scomberesox ${ }^{250}$ Lacépède, 1803, 67.

Sayris Rafinesque, 1810,81 .

Grammiconotus Achille Costa, 1862, 312.
Cololabis Gill, 1896, 473.

SCOM Beressus Jordan, 1920, 571.

${ }^{250}$ Often written Scombresox.

\section{Family 304. HEMIRAMPHID无}

(Half-beaks)

Hemi-Ramphus ${ }^{257}$ Cuvier, 1817, 99.

Dermogenys ${ }^{258}$ Van Hasselt, 1823, 172.

Cobitopsis Pomel, 1853, 254.

Hyporhamphus Gill, 1859, 289.

Euleptorhamphus Gill, 1859, 289, 326.

Zenarchopterus Gill, 1863, 326.

Oxyporhamphus Gill, 1863, 326.

Hemirhamphodon Bleeker, 1866, 340.

Arrhamphus Günther, 1866, 340.

Hemila m pronites Geinitz, 1868, 350.
Chriodorus Good'e \& Bean, 1882, 419.

Hemiexocœtus Fowler, 1901, 493.

Labidorhamphus Fowler, 1905, 513.

Eulepidorhamphus Fowler, 1919.

Rogenites Jordan, 1919, 568.

ZeLosis Jordan \& Gilbert, 1920, 571.

Rhamphodermogenys ${ }^{258}$ Fowler \& Bean, 1922.

Nomarhamphus Weber, 1922 (See Index A).

${ }^{257}$ Usually spelled Hemirhamphus.

${ }^{258}$ Spelled Dermatogenys by Bleeker.

${ }^{258 a}$ Loc. cit., 7 ; orthotype Hemirhamphus sajori Schlegel.

${ }^{250}$ Fowler \& Bean, "Fishes of Formosa and the Philippine Islands," Proc. U. S. Nat. Mus.; 152: 17; orthotype Dermogenys bakeri Fowler \& Bean.

\section{Family 305. EXOCCETID尼}

\section{(Flying Fishes)}

Exocœtus Linnæus, 1758, 15 .

Hirundo Catesby, 1771, 30 .

Pterichthus (Commerson) Lacépède, $1803,71$.

Cypselurus $^{200}$ Swainson, 1839, 203.

Cheilopogon Lowe, 1840, 207.

Ptenichthys Müller, 1843, 216.
Halocypselus Weinland, 1858, 284.

Parexocœtus Bleeker, 1866, 340.

Fodiator Jordan \& Meek, 1885, 433.

Exonautes Jordan \& Evermann, 1896, 472.

Evolantia Heller \& Snodgrass, 1903, 503.

Ptenonotus Ogilby, 1908, 529, 554.

${ }^{200}$ Spelled Cypsilurus by misprint, as decided by the International Commission of Nomenclature. 


\section{Order ANACANTHINI}

This large group, usually associated with the blennies and brotulids on the one hand and the flounders on the other, is quite distinct from both. Its origin and relationship are obscure. The absence of fin spines and the frequent presence of more than five ventral rays indicate its descent from some early type in which the ventral rays have not been reduced to I, 5, the normal number in percomorphous fishes, and no spines have developed in the fins. The anterior position of the ventrals separate these fishes from the forms with a duct to the air bladder, Physostomi, Malacopteri, Ostariophysi, Isospondyli, etc. Regan suggests their possible derivation from allies of Aulopidce or other primitive Iniomi.

\section{Family 306. MACROUROIDID $\approx$}

Macrouroides Smith \& Radicliffe, 1912, Squalogadus Gílbert \& Hubbs, 1916, 559. 544.

Family 307. MACROURID五 (Coryphanoidida; Macrurida)

(Grenadiers; Rat-tails)

Coryphænoides Gunner, 1761, 16.

Macrourus $^{231}$ Bloch, 1786, 44.

Trachyrincus ${ }^{202}$ Giorna, 1805, 74, 407.

Colorhynchus Giorna, 1805, 74.

Lepidoleprus Risso, 1810, 77.

Oxycephas Rafinesque, 1810, 79.

Krohnius Cocco, 1844, 219.

Coccolus (Kaup) Bonaparte, 1844, 219, 226, 284.

Lepturus Gronow, 1854, 259.

Malacocephalus Günther, 1862, 318.

Macruronus Günther, 1872, 365 .

Macruroplus Bleeker, 1874, 372 .

Paramacrurus Bleeker, 1874, 372.

Oxymacrurus Bleeker, 1874, 372.

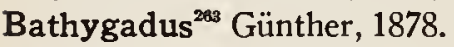

Lepidorhynchus Bleeker, 1879, 398.

Chalinura $^{284}$ Goode \& Bean, 1882, 419.

Hymenocephalus Giglioli \& Issel, 1884, 575.

Trachonurus Günther, 1887, 437.

Lionurus Günther, 1887, 437.

Mystaconurus Günther, 1887, 437.
Optonurus Günther, 1887, 437.

Nematonurus Günther, 1887, 437.

Cetonurus Günther, 1887, 437.

Ly conus Günther, 1887, 437.

Steindachneria Goode \& Bean, 1888, 441.

Abyssicola Goode \& Bean, 1895, 468.

Moseleya Goode and Bean, 1895, 468.

Steindachnerella Eigenmann, 1897, 476.

Cœlocephalus Gilbert \& Cramer, 1897, 477.

Albatrossia Jordan \& Gilbert, 1898, 482.

Bogoslovius J. \& E., 1898, 482.

Matæocephalus Berg, 1899, 484.

Dolloa Jordan, 1900, 489.

Gadomus Regan, 1903,.505.

Melanobranchus Regan, 1903, 505.

Nezumia Jordan \& Gilbert, 1904, 509.

Regania Jordan \& Gilbert, 1904, 509.

Cynomacrurus Dollo, 1909, 532.

Ateleobrachium $^{205}$ Gilbert \& Burke, 1912, 543.

Echinomacrurus Roule, 1916, 560.

Lyconodes Gilchrist, 1922. (See Index.)

${ }^{201}$ Usually corrected as Macrurus.

${ }^{203}$ Usually corrected to Trachyrhynchus.

${ }^{203}$ Ann. Mag. Nat. Hist., 23; orthotype Bathygadus cottoides Günther. (Omitted in "Genera of Fishes.")

${ }^{204}$ Written Chalinurus by Günther.

${ }^{205}$ Bull. U. S. Bur. Fish., $30: 94,1912$. (Erroneously given in "Genera of Fishes.") 
Hyomacrurus Gilbert \& Hubbs, 1920. Quincuncia G. \& H., 1920, 570.

(See Index.)

Hymenogadus G. \& H., 1920, 570.

Hyostomus Gilbert \& Hubbs, 1920, 570.

Atherodon G. \& H., 1920, 570.

Oxygadus G. \& H., 1920, 570 .

Ventrifossa G. \& H., 1920, 570.

Papyrocephalus G. \& H., 1920, 570.

Lucigadus G. \& H., 1920, 570.

Lucigadella G. \& H., 1920, 570.

\section{Family 308. BREGMACEROTID $\mathbb{E}$}

Bregmaceros (Cantor) Thompson, 1840, 208.

Asthenurus Tickell, 1865, 339,

Calloptilum Richardson, 1844, 222.

Auchenoceros Günther, 1889, 447.

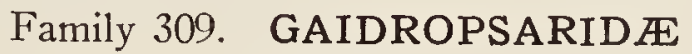

(Rocklings)

Enchelyopus Bloch \& Schneider, 1801, 57.

Gaidropsarus Rafinesque, $1810,81$.

Dropsarus Rafinesque, 1815, 88.

Onos Risso, 1826, 119.

Ciliata Couch, 1832, 176.

Couchia Thompson, 1856, 274.

Molvella Kaup, 1858, 281.

Motella Cuvier, 1829, 131.

Rhinonemus Gill, 1863, 325.

\section{Family 310. GADID $Æ$}

(Cod-fishes)

Gadus Linnæus, 1758, 12.

Callarias Klein, 1777, 42.

Phycis Röse, 1793, 51.

Phycis Bloch \& Schneider, 1801, 58.

Phycis Rafinesque, 1810, 79.

Strinsia Rafinesque, $1810,81$.

Eleginus Fischer, 1813, 85.

Trisopterus Rafinesque, 1814, 87.

Morrhua (Cuvier) Oken, 1817, 100.

Merlangus (Cuvier) Oken, 1817, 100.

Raniceps (Cuvier) Oken, 1817, 100.

Lota (Cuvier) Oken, 1817, 100.

Mustela (Cuvier) Oken, 1817, 100.

Brosme $^{286}$ (Cuvier) Oken, 1817, 100.

Lotta Risso, 1826, 119.

Morua Risso, 1826, 119.

Mora Risso, 1826, 119.

Molva Fleming, 1828, 122.

Brosmus Stark, 1828, 123.

Tilesia Swainson, 1839, 203.

Cephus Swainson, 1839, 203.

Lepidion Swainson, 1839, 203.

Gadella Lowe, 1843, 215.

Nemopteryx Agassiz, 1844, 219.

Asellus Valenciennes, 1844, 223.

Merlinus Agassiz, 1845, 224.
Pollachius (Nilsson) Bonaparte, 1845, 226.

Gastronemus Bonaparte, 1845, 226.

Tinimogaster Bonaparte, 1845, 226.

Uraleptus Costa, 1846, 574.

Cerdo Gistel, 1848, 235.

Gadiculus Guichenot, 1850, 246.

Lotella Kaup, 1858, 281.

Physiculus Kaup, 1858, 281.

Uraleptus Costa, 1858, 279.

Pteridium Filippi \& Verany, 1859, 289.

Palasobrosmius Rath, 1859, 292.

Palæogadus Rath, 1859, 292.

Hypsiptera Günther, 1860, 297.

Algoa Castelnau, 1861, 301.

Melanogrammus Gill, 1862, 316.

Brachygadus Gill, 1862, 316.

Læmonema Günther, 1862, 318.

Pseudophycis Günther, 1862, 318 .

Halargyreus Günther, 1862, 318.

Boreogadus Günther, 1862, 318.

Haloporphyrus Günther, 1862, 318.

Urophy cis Gill, 1863, 325.

Micromesistius Gill, 1863, 325.

Brachygadus Gill, 1863, 325 .

Leptogadus Gill, 1863, 325.

\footnotetext{
${ }^{260}$ Later written Brosmius by Cuvier.
} 
Odontogadus Gill, 1863, 325.

Microgadus Gill, 1863, 325.

Eglefinus Malm, 1877, 391.

Antimora ${ }^{267}$ Günther, 1878.

Melanonus Günther, 1878, 394.

Pleurogadus Bean, 1885, 433.

Salilota Günther, 1887, 437.

Brosmiculus Vaillant, 1888, 444.

Sympodoichthys Facciolà, 1888, 441.

Austrophycis Ogilby, 1897, 477.

Emphycus Jordan \& Evermann, 1898, 481.

Theragra Lucas, 1898, 481.

Microlepidium Garman, 1899, 486.
Leptophycis Garman, 1899, 486.

Rhinocephalus (Agassiz) Woodward, 1901, 223.

Tripterophycis Boulenger, 1902, 497.

Melanonosoma Gilchrist, 1902, 498, 501.

Læmonemodus Gilchrist, 1903, 503.

Gargilius $^{203}$ (Jensen) Schmidt, 1906.

Arnoldina Jordan \& Gilbert, 1919, 569.

ECLipes J. \&. G., 1919, 569.

Merria mina J. \& G., 1919, 569.

Verater Jordan, 1919, 567.

ARNOLdites ${ }^{269}$ Jordan, 1923.

\footnotetext{
${ }^{207}$ Ann. Mag. Nat. Hist., 2: 18; orthotype A. rostrata Günther. (Omitted in "Genera of Fishes.")

${ }^{288}$ Gargilius (Jensen, Adolf Severien) Schmidt (Ernest Johannes), "Contrib. Life Hist. Eel," 177, 1906; no description and no specific name given; a small deep-water gadoid. (See Holt \& Byrne, Fish. Ireland Invest., 5 : 58.)

${ }^{260}$ A new generic name to replace Arnoldina, preoccupied in Unionida (Hannibal, 1917).
}

\section{Family 311. MERLUCCIIDE} (Hakes)

Merluccius $^{270}$ Rafinesque, $1810,79$.

Onus Rafinesque, 1810,81 .

Merlangus Rafinesque, 1810,83 .

Stomodon Mitchill, $1814,86$.

Hydronus Minding, 1832, 176.
Merlus Guichenot, 1848, 238.

Merlucius Gronow, 1854, 259.

Homalopomus Girard, 1856, 269.

Epicopus Günther, 1860, 296.

${ }^{270}$ Often spelled Merlucius.

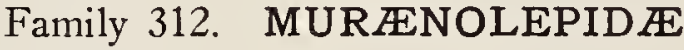

Murænolepis Günther, 1880, 403.

\section{Family 313. ERETMOPHORID平}

Hypsirhynchus Facciolà, 1884, 427, 468. Eretmophorus Giglioli, 1889, 446.

\section{Order SALMOPERCA}

Adipose fin present; dorsal fin with spines.

The Salmoperce and Xenarchi are detached fragments of a lost fresh water fauna of the Cretaceous or Eocene. They are closely related to each other, but neither has much affinity with other known fishes. The several orders which follow are often spoken of as transitional between soft-rayed and spiny-rayed fishes. The word is misleading. More accurately they are diverging offshoots from transitional forms now extinct; no linear series can express their varied relations, their line of evolution in some cases leading distinctly downward, that is, toward less 
than ancestral specialization. Some groups as the Percesoces that seem to lead upward toward the Percoidei may really be declining downward from perch-like ancestry.

In general the forward movement is marked by the development of spines in the fins, the thoracic attachment of the ventral fins, the reduction in the number of ventral rays to six, the first being normally spinous, the loss of the mesocoracoid, the relegation of the maxillary to a subordinate position in the upper jaw, the roughening of the scales, the obliteration of the duct to the air bladder, with a number of minor characters. These transitions do not appear uniformly, and they are sometimes disguised by special traits of degeneration among the varied groups naturally regarded as Acanthopterygians or spiny-rayed. But nowhere in the vast range of variety among fishes is it possible to arrange a linear series which shall express the actual fact. The most one can do is keep up a sort of marching abreast from the Ganoids, through the Isospondyli and transitional types to the specialized Acanthopteri and their peculiar offshoots. In this group and the next, which is closely related, the pneumatic duct is obsolete; dorsal and anal each with a few spines; ventral rays more than five.

\section{Family 314. PERCOPSID瓜}

(Sand Rollers; Trout Perch)

Adipose fin present; ventrals subabdominal, without spine.

Percopsis Agassiz, 1848, 232.

Columbia Eigenmann, 1892, 457.

Salmoperca Thompson, 1851, 249.

\section{Order XENARCHI}

Adipose fin wanting; ventrals subthoracic, with a spine.

\section{Family 315. APHREDODERID压} (Pirate Perch)

Aphredoderus ${ }^{271}$ Cuvier \& Valenciennes, $\quad$ Sternotremia Nelson, 1876, 386. 1833, 178.

ERISmatopterus Cope, 1870,356 .

Asternotremia Nelson, 1877, 390.

Trichophanes Cope, 1872, 364.

Amphiplaga Cope, 1877, 388.

${ }^{271}$ Corrected to Aphododerus and Aphrodedirus by authors.

\section{Family 316. ASINEOPID $\mathbb{E}$}

Asingops Cope, 1872, 356.

\section{Order ALLOTRIOGNATHI}

Suborder HISTICHTHYES

Family 317. VELIFERID压

Velifer Temminck \& Schlegel, 1850, 247. 


\section{Family 318. LOPHOTID王}

Lophotes Giorna, 1805, 74.

Leptopus Rafinesque, 1814, 86.
Podoleptus Rafinesque, 1815, 92.

Eumecichthys $^{272}$ Regan, 1907.

${ }^{272}$ Proc. Zool. Soc. Lond., 638, 1907 ; orthotype Lophotes fiskii Günther. (Omitied in "Genera of Fishes.")

\section{Suborder TAENIOSOMI}

Family $319 . \quad$ REGALECID㤅

(Oar Fishes; Sea Serpents)

Regalecus Brünnich, 1771, 28.

Regalecus Ascanius, 1788, 45.

Gymnetrus Bloch, 1788, 45.

Cephalepis Rafinesque, 1810, 82.
Xypterus Rafinesque, 1810, 82.

Epidesmus Ranzani, 1818, 107.

Xiphichthys Swainson, 1839, 201.

\section{Family 320. TRACHIPTERID瓜}

(King-of-the-Herring)

Trachipterus ${ }^{273}$ Gouan, 1770, 28.

Gymnogaster Brünnich, 1788, 168.

Bogmarus Bloch \& Schneider, 1801, 60.

Argyctius Rafinesque, 1810, 80.
Nemochirus Rafinesque, 1815, 86, 407.

Nemotherus Costa, 1834, 180.

Vogmarus Reid, 1849, 244.

${ }^{273}$ Usually corrected to Trachypterus.

\section{Suborder ATELAXIA}

Family 321. STYLEPHORIDE

Stylephorus Shaw, 1791, 50.

Gigantura Brauer, 1901, 492.

\section{Family 322 GIGANTURID $Æ$}

\section{Order SELENICHTHYES}

Ventrals many-rayed; basal bones of pectoral set horizontally; humeral arch and pelvic bones enormously enlarged; body very deep, compressed.

\section{Family 323. LAMPRID}

(Opah; Moon-fish)

Lampris Retzius, 1799, 54.

Chrysotosus Lacépède, 1803, 65.

Echemythes Gistel, 1848, 235.
Diatom ceCA Jordan \& J. Z. Gilbert, 1919, 569.

\section{Family 324. SEMIOPHORID $\mathbb{E}$}

Sem iophorus Agassiz, 1838, 191.

\section{Order HETEROSOMATA}

(Flounders; Flat Fishes)

Both eyes on the same side of the body.

The flounders and soles, having no spines and the ventral fins thoracic 
with an increased number of rays, should not be placed far from the percomorphous series. Boulenger suggests that the group may have close affinities with the Amphistiida and Zeida, constituting with these the order Zeorhombi. This view of the case is plausible, but it is far from established.

\section{Family 325. PSETTODIDA}

Psettodes Bennett, 1830, 174.

Sphagomorus Cope, 1867, 344.

\section{Family 326. BOTHIDE}

Solea Catesby, 1771, 31.

Psetta Klein, 1775, 38.

Europus Klein, 1775, 38.

Rhombus Klein, 1775, 38.

Citharus Röse, 1793, 52.

Bothus Rafinesque, 1810, 79.

Scophthalmus Rafinesque; $1810,82$.

Rhombus Cuvier, 1817, 100.

Rhomboides Goldfuss, 1820, 171.

Zeugopterus Gottsche, 1835, 182.

Citharus Reinhardt, 1838, 193.

Psetta Swainson, 1839, 203.

Platophrys Swainson, 1839, 203.

Syacium Ranzani, 1840, 207.

Peloria Cocco, 1844, 219.

Passer Valenciennes, 1855, 266.

Rhomboidichthys Bleeker, 1856, 267.

Lophopsetta Gill, 1861, 303, 331.

Citharus Bleeker, 1862, 310 .

Hemirhombus Bleeker, 1862, 310 .

Citharichthys Bleeker, 1862, 310 .

Arnoglossus Bleeker, 1862, 310 .

Orthopsetta Gill, 1862, 317.

Engyprosopon Günther, 1862, 319.

Phrynorhombus Günther, 1862, 319.

Lepidorhombus Günther, 1862, 319.

Metoponops Gill, 1864, 331.

Bascanius $^{274}$ Schiödte, 1868, 353.

Nematops Günther, 1880, 403.

Lophonectes Günther, 1880, 403.

Monolene Goode, 1880, 403.

Thyris Goode, 1880, 403.
Anticitharus Günther, 1880, 403.

Læops Günther, 1880, 403.

Thysanopsetta Günther, 1880, 403.

Lepidopsetta Günther, 1880, 403.

Etropus Jordan \& Gilbert, 1881, 416.

Lophorhombus Macleay, 1882, 421.

Delothyris Goode, 1883, 424.

Charybdia Facciolà, 1885, 431.

Aramaca Jordan \& Goss, 1885, 433.

Cyclopsetta Gill, 1888, 441.

Azevia Jordan \& Goss, 1888, 442.

Eucitharus Gill, 1888, 441.

Trichopsetta Gill, 1888, 441.

Engyophrys Jordan \& Bollman, 1889, 447.

Psettylis Alcock, 1890, 450.

Scianectes Alcock, 1890, 450.

Caulopsetta Gill, 1893, 461.

Chascanopsetta Alcock, 1894, 463.

Embassichthys Jordan \& Evermann, $1896,475$.

Boopsetta Alcock, 1896, 470.

Pelecanichthys Gilbert \& Cramer, 1897, 477.

Perissias Jordan \& Evermann, 1898, 481.

Apsetta Kyle, 1900, 490.

Scæops ${ }^{275}$ Jordan and Starks, 1904.

Tæniopsetta Gilbert, 1905, 513.

Mancopsetta Gill, 1905, 514.

Lambdopsetta Smith \& Pope, 1906, 520.

Trachypterophrys ${ }^{278}$ Franz, 1910.

Plagiopsetta Franz, 1910, 535.

Laiopteryx $x^{277}$ Weber, 1913, 551.

${ }^{274}$ An undetermined larva.

${ }^{275}$ Sccoops Jordan \& Starks, loc. cit., 623; orthotype Rhombus grandisquama Temminck \& Schlegel.

${ }^{270} \mathrm{Op}$. cit., 60; orthotype $P$. raptator Franz; a synonym of Chascanopsetta Alcock, according to McCulloch. (Omitted in "Genera of Fishes.")

${ }^{277}$ Misprinted Liopteryx in "Genera of Fishes." 
Pseudocitharichthys ${ }^{278}$ Weber, 1913.

Lepidoblepharon Weber, 1913, 551.

Eовотнus Eastman, 1914, 552.

Læoptichthys Hubbs, 1915, 556.

Citharoides Hubbs, 1915, 556.

Psettina Hubbs, 1915, 556.
Asterorhombus Tanaka, 1915, 558.

Scidorhombus Tanaka, 1915, 558.

ZoRorнombus Jordan, 1920, 571.

Paracitharus ${ }^{279}$ Regan, 1920.

Crossorhombus ${ }^{280}$ Regan, 1920.

Platotichthys ${ }^{281}$ Nichols, 1921.

${ }^{278}$ Op. cit. 413 ; orthotype $P$. aureus Weber. (Omitted in "Genera of Fishes.")

zтв "A Review of the Flat Fishes (Heterosomata) of Natal," Ann. Durban Museum, 2: 209, March 25, 1920; type Arnoglossus macrolepis Günther.

${ }^{280}$ Regan, op. cit., 211 ; type Platophrys dimorphus Günther.

${ }^{281}$ "List of Turks Island Fishes," Bull. Amer. Mus. Nat. Hist., $44: 21$; orthotype $P$. chartes Nichols.

\section{Family 327. PARALICHTHYIDE \\ (Bastard Halibuts)}

To the Bothida Regan refers all sinistral or "left-handed" flounders. The genera named below, however, seem to me quite as closely related to the Hippoglossidce as to Bothus and Platophrys. They may be allowed to stand as a separate transitional subfamily or family pending further investigation.

Paralichthys ${ }^{232}$ Girard, 1859.

Chænopsetta Gill, 1861, 303.

Uropsetta Gill, 1862, 317.

Pseudorhombus Bleeker, 1862, 310 .

Tephritis Günther, 1862, 318.

Tephrinectes Günther, 1862, 319.

Ancylopsetta Gill, 1864, 331.

Neorhombus Castelnau, 1875, 378.

Hippoglossina Steindachner, 1876, 387.

Teratorhombus Macleay, 1881, 416 .
Notosema Goode \& Bean, 1882, 419.

Xystreurys Jordan \& Gilbert, 1890, 451.

Lioglossina Gilbert, 1890, 451.

Verecundum Jordan, 1890, 452.

Gastropsetta Goode \& Bean, 1894, 463.

Ramularia Jordan \& Evermann, 1898, 481.

Rhombiscus Jordan \& Snyder, 1900, 490.

Evestues J. Z. Gilbert, 1905, 536.

Velifracta Jordan, 1907, 524.

Tarphops Jordan \& Thompson, 1914, 553.

${ }^{282}$ Omitted from No. 657, "Genera of Fishes"; orthotype Paralichthys maculosus Girard.

\section{Family 328. HIPPOGLOSSID $\mathbb{E}$}

(Halibuts)

Hippoglossus Cuvier, 1817, 100.

Hippoglossoides Gottsche, 1835, 182.

Reinhardtius Gill, 1861, 302.

Drepanopsetta Gill, 1861, 303.

Platysomatichthys Bleeker, 1862, 310.

Pomatopsetta Gill, 1864, 331.

Atheresthes Jordan \& Gilbert, 1880, 404.

Eopsetta Jordan \& Goss, 1885, 433.

Lyopsetta Jordan \& Goss, 1885, 433.
Verasper Jordan \& Gilbert, 1898, 481.

Cynopsetta Schmidt, 1903, 506, 519.

Xystrias ${ }^{283}$ Jordan \& Starks, 1904.

Veræqua J. \& S., 1904, 509.

Dexistes J. \& S., 1904, 509.

Araias J. \& S., 1904, 509.

Cleisthenes J. \& S., 1904, 509.

Protopsetta Schmidt, 1904, 511.

Acanthopsetta Schmidt, 1904, 511.

${ }^{283}$ Loc. cit., 623; orthotype Hippoglossus grigorjewi Herzenstein. (Omitted in "Genera of Fishes.") 


\section{Family 329. PLEURONECTID EE}

Pleuronectes Linnæus, 1758, 13.

Passer Klein, 1775, 38.

Platessa Cuvier, 1817, 100.

Microstomus Gottsche, 1835, 182.

Limanda Gottsche, 1835, 182.

Glyptocephalus Gottsche, 1835, 182.

Cynicoglossus Bonaparte, 1837, 187.

Cynoglossa Bonaparte, 1845, 226.

Platichthys Girard, 1854, 257.

Psettichthys Girard, 1854, 257.

Parophrys Girard, 1854, 257.

Pleuronichthys Girard, 1854, 257.

Hypsopsetta Gill, 1862, 575, 317.

Lepidopsetta Gill, 1862, 575, 317 .

Myzopsetta Gill, 1861, 308, 331.

Clidoderma Bleeker, 1862, 310.
Pseudopleuronectes Bleeker, 1862, 310.

Heteroprosopon Bleeker, 1862, 310.

Brachyprosopon Bleeker, 1862, 310.

Liopsetta Gill, 1864, 331.

Euchalarodus Gill, 1864, 331.

Pœcilopsetta Günther, 1880, 403.

Flesus Moreau, 1881, 416.

Isopsetta Lockington, 1882, 420.

Inopsetta Jordan \& Goss, 1885, 433.

Kareius Jordan \& Snyder, 1900, 490.

Alæops Jordan \& Starks, 1904, 509.

Limandella Jordan \& Starks, 1906, 519.

Gareus Hubbs, 1915, 556.

Pluviopsetta Tanaka, 1916, 560.

Tanakius Hubbs, 1918, 564.

Errex Jordan, 1919, 567.

Family 230. SAMARID压 (Paralichthodida)

Samaris Gray, 1831, 139.

Brachypleura Günther, 1862, 319.
Paralichthodes Gilchrist, 1902, 498.

Samariscus Gilbert, 1905, 513.

\section{Family 331. RHOMBOSOLEIDEE}

Psammodiscus Günther, 1862, 319.

Rhombosolea Günther, 1862, 319.

Ammotretis Günther, 1862, 319.
Peltorhamphus Günther, 1862, 319.

Oncopterus Steindachner, 1875, 381.

\section{Family 332. ACHIRID五}

Eyes and color dextral. Ventral of the right side continuous along the ridge of the abdomen and joined to the anal; caudal free from dorsal and anal.

Achirus Lacépède, 1803, 65.

Trinectes Rafinesque, 1832, 142.

Apionichthys Kaup, 1858, 282.

Soleotalpa Günther, 1862, 319.

Gymnachirus Kaup, 1858, 282.
Achiropsis Steindachner, 1876, 387.

Baiostoma Bean, 1882, 419.

Amate Jordan \& Starks, 1906, 519.

Soleonasus Eigenmann, 1912, 543.

Pnictes Jordan, 1919, 567.

\section{Family 333. SOLEID $\mathbb{E}$}

(Soles)

Eyes and color dextral; caudal free from dorsal and anal, as are the ventral fins also.

Solea Klein, $1775,40$.

Solea Quensel, 1806, 74.

Solea Rafinesque, 1810, 81.

Odontolepis Fischer, 1813, 85.

Monochirus Rafinesque, 1814, 87.

Solea Cuvier, 1817, 100.

Monochirus (Cuvier) Oken, 1817, 100.
Leptosoma Nardo, 1827, 121.

Monochir Cuvier, 1829, 131.

Monochirus Bonaparte, 1837, 141, 187.

Microchirus Bonaparte, 1837, 187.

Spanius Gistel, 1848, 236.

Monochirus Kaup, 1858, 282.

Aseraggodes Kaup, 1858, 282. 
Heteromycteris Kaup, 1858, 282.

Soleichthys Bleeker, 1860, 294.

Pegusa Günther, 1862, 319.

Microbuglossus Günther, 1862, 319.

Pardachirus Günther, 1862, 319.

Buglossus Günther, 1862, 319.

Liachirus Günther, 1862, 319.
Bowenia Haast, 1873, 369.

Quenselia Jordan, 1888, 442.

Pelotretis Waite, 1911, 541.

Phyllichthys McCulloch, 1916, 559.

Bathysolea Roule, 1916, 560.

Eusolea ${ }^{24}$ Roule, 1919.

${ }^{2 * 4}$ New name, perhaps not intended as a generic name distinct from Solea. Solea capellonis Steindachner the only species mentioned.

\section{Family 334. SYNAPTURID正}

Eyes dextral; ventrals distinct; dorsal and anal united with caudal.

Brachirus $^{285}$ Swainson, 1839, 203.

Synaptura Cantor, 1849, 241.

Achiroides Bleeker, 1851, 247.

Esopia Kaup, 1858, 282.

Euryglossa Kaup, 1858, 282.
Eurypleura Kaup, 1858, 282.

Anisochirus Günther, 1862, 319.

Zebrias Jordan \& Snyder, 1900, 490.

Austroglossus ${ }^{28}$ Regan, 1920.

${ }^{285}$ Not Brachyrus Swainson of page 71 . The name Brachirus is first used by Swainson, 1839, II, page 71 , for an ally of Pterois, with a brief diagnosis, no species being mentioned. On page 180 the same diagnosis is given without reference to species, the name being changed to Dendrochirus. On page 264 the definition is expanded, the word is spelled Brachyrus and two species, zebra and brachypterus, are mentioned. The first of these has been taken by the first reviser, Swain (1882), as type. On page 303 Swainson again uses the name Brachiruis, this time for a genus of Soles, mentioning five species, plagiusa, orientalis, zebra, commersoni (anus) jerreus, and pan.

Of these, plagiusa is a Symphurus, not related to the others and not agreeing with the generic diagnosis. In 1849, recognizing that the name Brachirus, which he changes to Brachyurus, was preoccupied, Cantor offered Synaptura as a substitute, at the same time redescribing commersonianus and zebra. Bleeker next described commersonianus as the sole species of Synaptura, referring the others to Brachirus, a plan inadmissable, as Synaptura was a substitute name for Brachirus, and thus subject to the same limitations. Kaup (1858) makes orientalis type of a new genus (Euryglossa) which Günther (1862) recognizes as a subgenus, leaving zebra, commersoniana and pan in Synaptura. Zebra is the type of Zebrias Jordan \& Snyder (1900); the two other species are congeneric, commersoniamus, after Bleeker, presumably serving as Günther's type. Swain, however, definitely named orientalis as type as the first species mentioned under Brachirus which corresponds to the author's definition. It is, however, more convenient as well as according better to the rules to regard commersonianus as type of Synaptura.

The name Brachirus must go with its first use, for Pterois zebra, thus replacing Dendrochirus.

${ }^{288} \mathrm{Op}$. cit., 217; orthotype Synaptura pectoralis Kaup; right pectoral longer than head; dorsal rays in increased number. 


\section{Family 335. CYNOGLOSSID $A$}

\section{(Tongue-fishes)}

Eyes and color sinistral; dorsal and anal joined to the caudal; pectorals wanting; ventrals, if present, free from anal.

Plagusia Browne, 1789, 46.

Symphurus Rafinesque, 1810, 81 .

Plagiusa Rafinesque, 1815, 88.

Plagusia (Browne) Cuvier, 1817. 101.

Cynoglossus Hamilton, 1822, 114.

Plagusia Swainson, 1839, 203.

Bibronia Cocco, 1844, 219.

Plagusia Bonaparte, 1845, 187, 226.

Euporista Gistel, 1848, 237.

Eupnœa Gistel, 1848, 238.

Cantoria Kaup, 1858, 282.

Grammichthys Kaup, 1858, 282.

Aphoristia Kaup, 1858, 282.
Icania Kaup, 1858, 282.

Trulla Kaup, 1858, 282.

Arelia Kaup, 1858, 282.

Glossichthys Gill, 1861, 303.

Ammopleurops Günther, 1862, 319, 278.

Paraplagusia Bleeker, 1865, 336.

Rhinoplagusia Bleeker, 1870, 355 .

Acedia Jordan, 1888, 442.

Usinosita ${ }^{287}$ Jordan \& Snyder, 1900, 490.

Areliscus Jordan \& Snyder, 1900, 490.

Cynoglossoides Von Bonde, 1922. (See Index.)

${ }^{287} \mathrm{Misprinted} U$ sinostia.

\section{Superorder ACANTHOPTER YGII}

\section{Order ZEOIDEI}

Family 336. ZEID E

(John Dories)

A family of uncertain relations, having perhaps some affinities with the flounders, with the increased number of ventral rays characteristic of the berycoid fishes, and the adnate post-temporal of Capros and the chætodonts.

Zeus Linnæus, 1758, 13.

Oreosoma Cuvier, 1829, 127.

Cyttus Günther, 1861,575 .

Cyttopsis Gill, 1862, 314.

Zenopsis Gill, 1862, 314.

Cyttordes Wettstein, 1886, 436.

Zenion Jordan \& Evermann, 1896, 473.

Rhombocyttus Gill, 1893, 461.

Capromimus Gill, 1893, 461.
Cyttosoma Gilchrist, 1903, 503, 504.

Zen Jordan, 1903, 504.

Stethopristes Gilbert, 1905, 513.

Cyttomimus Gilbert, 1905, 513.

Pseudocy ttus Gilchrist, 1906, 518.

Neocyttus ${ }^{288}$ Gilchrist, 1906.

Parazenopsis Cligny, 1909, 532.

Cyttula Weber, 1913, 551.

Allocyttus McCulloch, 1914, 554.

${ }^{28 s} \mathrm{Op}_{\text {. cit., }} 4: 153$; orthotype $N$. rhomboidalis Gilchrist. (Omitted in "Genera of Fishes.")

\section{Family 337. GRAMMICOLEPIDE}

Grammicolepis Poey, 1873, 371.

Vesposus ${ }^{280}$ Jordan, 1921.
Xenolepidichthys Gilchrist, 1922. (See Index.)

${ }^{289}$ Proc. U. S. Nat. Mus., $70: 649$; orthotype Vesposus egregius Jordan.

\section{Order XENOBERYCES}

\section{Family 338. STEPHANOBERYCID $\mathbb{E}$}




\section{Family 339. MELAMPHAID屃}

A small group of deep-sea fishes not altogether homogeneous.

Metopias Lowe, 1843, 215.

AulolePIS Geinitz, 1849, 408.

Anoplogaster Günther, 1859, 291.

Melamphaës Günther, 1864, 333.

Poromitra Goode \& Bean, 1881, 419.

Caulolepis Gill, 1883, 424.
Plectromus Gill, 1883, 424.

Scopelogadus Vaillant, 1888, 444.

Lophocephalus Osorio, 1906, 520.

Scopeloberyx Zugmayer, 1911, 541.

Poromitrella Zugmayer, 1911, 541.

\section{Order BERYCOIDEI}

\section{Family 340. POLYMIXIID无}

Polymixia Lowe, 1838, 192.

Nemobrama Valenciennes, 1844, 223.

PyCNOSTERINX ${ }^{200}$ Heckel, 1849, 243.
I MOGASTER Costa, 1857, 275.

Platycormus von der Marck, 1858, 283.

Dinemus Poey, 1860, 298.

${ }^{200}$ Misprinted in "Genera of Fishes." This genus and the two which follow are probably beryccid, but their relations are uncertain.

\section{Family 341. BERYCOPSIDE}

Berycopsis Dixon, 1850, 245.

Stenostoma Dixon, 1850, 245.

\section{Family 342. BERYCID压}

Beryx Cuvier, 1829, 127.

Pristigenys Agassiz, 1839, 191, 194.

Centroberyx Gill, 1862, 315.

MACROLEPIS von der Marck, 1863, 327, 346.

Heterolepis Costa, 1865, 336.

HEMigonolepis Steinla, 1868, 350.

Prionolepis Steinla 1868, 350.

Goniolepis Steinla, 1868, 350.
HeM ICyClolepis Steinla, 1868, 350.

SPINAcites Fritsch, 1893, 461.

Electrolepis Fritsch, 1893, 461.

Lobopterus Kramberger, 1895, 469.

Trachichthodes Gilchrist, 1903, 503.

Bradyurus Gill, 1904, 508.

Austroberyx McCulloch, 1911, 540.

\section{Family 343. HOPLOPTERYGID瓜}

Hoplopteryx Agassiz, 1838, 191.

\section{Family 344. DIRETMIDEE}

Diretmus Johnson, 1863, $326 . \quad$ Discus $^{201}$ Campbell, 1879, 399.

${ }^{201}$ A synonym of Dirctmus.

\section{Family 345. MONOCENTRIDE}

(Pine-cone Fishes)

Monocentris Bloch \& Schneider, 1801, 58 . Lepicantha Rafinesque, 1815, 89.

Lepisacanthus Lacépède, 1802, 62.

Ericius Tilesius, 1809, 76.

Cleidopus De Vis, 1883, 423.

\section{Family 346. ANOMALOPID正}

Heterophthalmus Bleeker, 1856, 267.

Anomalops Kner, 1868, 352.

Photoblepharon Weber, 1902, 501. 
Family 347. TRACHICHTHYID E

Trachichthys Shaw, 1799, 55.

Hoplostethus Cuvier \& Valenciennes, 1829, 133.

Acrogaster Agassiz, 1838, 191.

Sphenocephalus Agassiz, 1838, 191.
Aipichthys Steindachner, 1859, 293.

ACANTHOPHoRIA Kramberger, 1895, 469.

Paratrachichthys Waite, 1899, 488.

Gephyroberyx ${ }^{202}$ Boulenger, 1902, 497.

Leiogaster Weber, 1913, 551.

${ }^{202}$ Misprinted in "Genera of Fishes."

\section{Family 348. HOLOCENTRID $\mathbb{E}$}

(Soldier Fishes)

Holocentrus ${ }^{209}$ Gronow, 1763, 19.

Farer Forskål, 1775, 34.

Holocentrus (Gronow) Scopoli, 1777, 42.

Erythrimus $^{204}$ (Plumier) Lacépède, 1803, 72.

Corniger Agassiz, 1829, 132.

Myripristis Cuvier, 1829, 127.

Ostichthys (Langsdorff) Cuvier \& Valenciennes, 1829, 174.

Rhynchich thys Cuv. \& Val., 1831, 137.

Homonotus Dixon, 1850, 245.

Plectrypops Gill, 1862, 315.

Rhinoberyx Gill, 1862, 315 .

Rhamphoberyx Gill, 1863, 324.
Neomyripristis Castelnau, 1873, 367.

Holotrachys Günther, 1873, 369.

Neoniphon Castelnau, 1875, 378.

Harpage De Vis, 1884, 427.

Ostichthys (Langsdorff) Jordan \& Evermann, 1896, 473.

Flammeo J. \& E., 1898, 482.

Howella Ogilby, 1898, 483.

TraChich Th yoldes Woodward, 1902, 501.

Beanea Steindachner, 1902, 500.

Sargocentron Fowler, 1904, 507.

Adioryx Starks, 1908, 531.

Caproberyx Regan, 1911, 540.

${ }^{203}$ Often written Holocentrum.

${ }^{204}$ Also written Eritrimus by Plumier.

Family 349. DINOPTERYGID压

Dinopteryx Woodward, 1901, 496.

\section{Family 350. CTENOTHRISSID王}

Aulolepis Agassiz, 1844, 218.

Ctenothrissa Woodward, 1899, 488.

Family 351. CARISTIIDE

Caristius Gill \& Smith, 1905, 514.

Platyberyx Zugmayer, 1911, 541.

\section{Order THORACOSTEI \\ Suborder HEMIBRANCHII \\ (Phthinobranchii)}

Family 352. GASTEROSTEID $\mathbb{E}$

(Sticklebacks)

Gasterosteus Linnæus, 1758, 13.

Gasteracanthus Pallas, 1811, 84.

Spinachia Cuvier, 1817, 105.
Leiurus Swainson, 1839, 200.

Polycanthus Swainson, 1839, 200.

Apeltes Dekay, 1842, 210. 
Pungitius ${ }^{295}$ Coste, 1846.

Pygosteus (Brevoort) Gill, 1861, 302.

Gastræa Sauvage, 1874, 377.
Gasterostea Sauvage, 1874, 377.

Eucalia Jordan, 1876, 386.

Merriamella Jordan, 1907, 525.

${ }^{205}$ Coste, P., "Nidification des épinoches et des épinochettes," Mém. Savants Etrangers, Paris, 1846, 10:574-588. Quoted by Gill, "Smithsonian Report for 1905," 505, 1907; type Gasterosteus pungitius Linnæus; replaces Pygosteus Brevoort. I owe this note to William Converse Kendall, not having seen Coste's paper.

\section{Family 353. AULORHYNCHIDÆ}

Aulorhynchus Gill, 1861, 305.

Aulichthys Brevoort, 1862, 315 .

Auliscops Peters, 1866, 342.

\section{Family 354. PROTOSYNGNATHID王}

Protosyngnathus Von der Marck, 1876,

386.

\section{Suborder LOPHOBRANCHII}

\section{Family 355. SOLENOSTOMID\#}

Solenostomus ${ }^{298}$ Lacépède, $1803,67$.

Solenichthus ${ }^{207}$ Bleeker, $1865,336$.

Solenor h y chus Heckel, 1854, 259.

${ }^{200}$ Written Solenostoma by Rafinesque.

${ }^{297}$ Later written Solenostomatichthys by Bleeker.

Family 356. SYNGNATHIDE (Hippocampida)

(Pipe Fishes; Sea Horses)

The pipe fishes, elongate, with caudal fin, grade almost imperceptibly into the more specialized sea horses (Hippocampus), in which the caudal is wanting and the tail prehensile.

Syngnathus Linnæus, 1758, 16.

Acus P. L. S. Müller, 1767, 24.

Acus Valmont, 1791, 50.

Typhle Rafinesque, 1810, 78.

Siphostoma Rafinesque, 1810, 79.

Hippocampus Rafinesque, 1810, 79.

Nerophis Rafinesque, 1810, 82.

Hippocampus Leach, 1814, 85 .

Tiphlinus Rafinesque, 1815, 91.

Hippocampus Cuvier, 1817, 98.

Scyphius Risso, 1826, 119.

Nematosoma Eichwald, 1831, 176.

Acestra Jardine, 1831, 137.

Calamostoma Agassiz, 1833, 177.

Acus Swainson, 1839, 205.

Solegnathus ${ }^{208}$ Swainson, 1839, 205.

${ }^{209}$ Later spelled Solenognathus.

200 Spelled Stigmatophora by error.

${ }^{300}$ Spelled Corythroichthys by error.
Phyllopteryx Swainson, 1839, 205.

Hippichthys Bleeker, 1849, 241.

Syngnathoides Bleeker, 1851, 248.

Microphis Kaup, 1853, 254.

Hemimarsupium Kaup, 1853, 254.

Doryichthys Kaup, 1853, 254.

Chœroichthys Kaup, 1853, 254.

Stigmatopora ${ }^{298}$ Kaup, 1853, 254.

Doryrhamphus Kaup, 1853, 254.

Leptoichthys Kaup, 1853, 254.

Leptonotus Kaup, 1853, 253.

Ichthyocampus Kaup, 1853, 253.

Corythoichthys ${ }^{300}$ Kaup, 1853, 253.

Trachyrhampus Kaup, 1853, 253.

Halicampus Kaup, 1853, 253.

Gasterotokeus Heckel, 1853, 253. 
Acentronura Kaup, 1853, 253.

Cœlonotus Peters, 1855, 266.

Siphonostoma ${ }^{300 a}$ Kaup, 1856.

Hemithylacus Kaup, 1856, 272.

Solenognathus Bleeker, 1856, 268.

Haliichthys Gray, 1859.

Dermatostethus Gill, 1862, 317.

Pseudosyngnathus Kner, 1863, 327, 338.

Belonichthys Peters, 1868, 353.

Protocampus Günther, 1870, 357.

Entelurus Duméril, 1870, 357.

Nannocampus Günther, 1870, 357.

Urocampus Günther, 1870, 357.

Hymenolomus Duméril, 1870, 357.
Atelurus Duméril, 1870, 357.

Typhlus (Bibron) Bleeker, 1870, 357.

Osphyolax Cope, 1875, 379.

Penetropteryx Lunel, 1881, 416.

Acmonotus Philippi, 1896, 475.

Phycodurus Gill, 1896, 471.

Yozia Jordan \& Snyder, 1901, 495.

Castelnauina Fowler, 1907, 523.

Macleayina Fowler, 1907, 523.

Acanthognathus Duncker, 1912, 542.

Micrognathus Duncker, 1912, 542.

Apterygocampus Weber, 1913, 551.

Histiogamphelus McCulloch, 1914, 553.

Lissocampus ${ }^{302}$ Waite \& Hale, 1921.

${ }^{800}$ Op. cit., 46; orthotype Syngnathus typhle Linnæus. A synonym of Typhle. (Omitted in "Genera of Fishes.")

${ }^{302}$ Records South Australian Museum, 1 : 306, Jan. 29, 1921 ; orthotype L. caudalis Waite \& Hale.

\section{Order HYPOSTOMIDES}

\section{Family 357. PEGASIDE}

(Sea Moths)

Pegasus Linnæus, 1758, 16.

Cataphractus Gronow, 1763, 21.

Eurypegasus Bleeker, 1863, 322.

Parapegasus Bleeker, 1870, 357.
Leptopegasus Bleeker, 1873, 367.

Zalises Jordan \& Snyder, 1901, 494.

Acanthopegasus McCulloch, 1915, 556.

\section{Order AULOSTOMI}

\section{Family 358. AULOSTOMIDE}

(Trumpet Fishes)

Aulostomus ${ }^{802}$ Lacépède, 1803, 66.

Polypterichthys Bleeker, 1853, 252.

${ }^{802}$ Often written Aulostoma.

\section{Family 359. FISTULARIID压}

\section{(Cornet Fishes)}

Fistularia Linnæus, 1758, 14.

Solenostomus Gronow, 1763, 21.

Petimbuabo Catesby, 1771, 31.

Solenostomus Klein, 1778, 43.

Solenostomus Browne, 1789, 46.
Aulus (Commerson) Lacépède, 1803, 70.

Cannorhynchus Cantor, 1849, 241.

Flagellaria ${ }^{303}$ Gronow, 1854.

Solenostomus (Gronow) Gill, 1861, 302.

Protaulopsis Woodward, 1901, 496.

${ }^{{ }^{809}}$ Type F. fistularis Gronow, F. tabacaria Linnæus. (Omitted in "Genera of Fishes.")

Family 360. UROSPHENID

Urosphen Agassiz, 1844, 181, 218.

\section{Family 361. RHAMPHOSID瓜}

Rhamphosus Agassiz, 1844, 218. 
Family 362. CENTRISCID 2 (Amphisilida)

Centriscus Linnæus, 1758, 16.

Acentrachme Gill, 1862, 315.

Amphisilen Klein, 1775, 38.

Aulorhamphus Zigno, 1890, 453.

Amphisile Cuvier, 1817, 106.

Aoliscus Jordan \& Starks, 1902, 499.

\section{Family 363. MACRORHAMPHOSID $\mathbb{E}$}

Macrorhamphosus Lacépède, 1803, 66.

Limiculina Fowler, 1907, 523.

Macrognathus Gronow, 1854, 259.

Notopogon Regan, 1914, 555.

Orthichthys Gill, 1862, 315 .

Centriscops Gill, 1862, 315.

Scolopacichthys Regan, 1914, 555.

\section{Order LABYRINTHICI}

\section{Family 364. LUCIOCEPHALIDE}

Diplopterus $^{304}$ Gray, 1831, 138 .

Luciocephalus Bleeker, 1851, 247.

${ }^{304}$ This name is preoccupied; the genus is identical with Luciocephalus.

Family 365. OPHICEPHALID $\mathbb{E}$

Channa Gronow, 1763, 22.

Chrama (Gronow) Scopoli, 1777, 42.

${ }^{300}$ Usually spelled Ophiocephalus.

\section{Family 366. HELOSTOMID 王}

Helostoma (Kuhl) Cuvier, 1829, 129.

Spirobranchus Cuvier, 1829, 129.

\section{Family 367. POLYACANTHID正}

Polyacanthus (Kuhl) Cuvier, 1829, 129, 173.

\section{Family 368. OSPHRONEMID $\mathbb{E}$}

Osphronemus ${ }^{308}$ Lacépède, 1801, 61.

Trichogaster Bloch \& Schneider, 1801, 58.

Trichopodus Lacépède, 1802,61.

Macropodus ${ }^{807}$ Lacépède, 1802, 63.

Platypopus 'Lacépède, 1804, 169.

Trichopus Shaw, 1804, 73.

Colisa Cuvier \& Valenciennes, 1831, 137.

Ctenops McClelland, 1845, 227.

Pedites Gistel, 1848, 236.
Lithulcus Gistel, 1848, 237.

Betta Bleeker, 1850, 245.

Trichopsis Canestrini, 1860, 295.

Sphærichthys Canestrini, 1860, 295.

Parosphromenus Bleeker, 1879, 398.

Pseudosphromenus Bleeker, 1879, 398.

Micracanthus Sauvage, 1879, 400.

Parophiocephalus ${ }^{308}$ Popta, 1905, 515.

Oshimia Jordan, 1919, 567.

\footnotetext{
${ }^{808}$ Written Osphromenus by authors.

${ }^{307}$ Written Macropus by authors.

${ }^{208}$ A synonym of Betta.
}

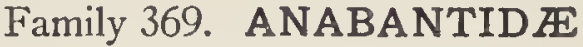

(Climbing Perch)

Anabas Cuvier, 1817, 106.

Ctenopoma Peters, 1846, 225.

Coius Hamilton, 1822, 114. 


\section{Order PERCOMORPHI}

\section{Suborder PERCESOCES}

\section{Family 370. NANNATHERINID $\mathbb{E}$}

Nannatherina ${ }^{800}$ Regan, 1906.

${ }^{800}$ Ann. Mag. Nat. Hist., 17: 451; orthotype N. ralstoni Regan. (Omitted in "Genera of Fishes.")

\section{Family 371. BEDOTIID $\approx$}

Bedotia Regan, 1903, 506.

\section{Family 372. MELANOT ENIID}

A group of river'fishes of the Australian region, closely related to the Atherinidce but showing certain traits which set them off as a distinct subfamily. The number of nominal genera had been inordinately multiplied.

Melanotænia Gill, 1862, 316.

Pseudomugil Kner, 1864, 334.

Strabo Kner \& Steindachner, 1866, 341.

Nematocentris Peters, 1870, 356.

Atherinosoma Castelnau, 1872, 363.

Zantecla Castelnau, 1873, 367.

Neoatherina Castelnau, 1875, 378.

Aida Castelnau, 1875, 378.

Aristeus Castelnau, 1879, 393, 399.
Rhombatractus ${ }^{810}$ Gill, 1894, 464.

Telmatherina Boulenger, 1897, 476.

Glossolepis Weber, 1907, 527.

Rhombosoma Regan, 1914, 554.

Rhadinocentrus Regan, 1914, 554.

Anisocentrus Regan, 1914, 554.

Centratherina ${ }^{811}$ Regan, 1914.

Chilatherina Regan, 1914, 554.

${ }^{810}$ The date 1879 on page 399, "Genera of Fishes," is an error.

${ }^{2 u}$ Regan, loc. cit.; orthotype Rhombatractus crassispinosus Weber. (Omitted in "Genera of Fishes.")

\section{Family 373. ATHERINID正}

(Silversides; Hardy Heads; Pescados del Rey; Peixerey)

Atherina Linnæus, $1758,15$.

Aphia ${ }^{\text {s11a }}$ Risso, 1826, 119.

Hepsetia Bonaparte, 1837, 187.

Menidia Bonaparte, 1837, 187.

Membras Bonaparte, 1837, 142, 187.

Chirostoma Swainson, 1839, 200.

Argyrea Dekay, 1842, 210.

Rhamphognathus Agassiz, 1844, 219.

Mesogaster Agassiz, 1844, 219.

Atherinoides Bleeker, 1853, 253.

Atherinichthys Bleeker, 1853, 253.

Heterognathus Girard, 1854, 258.

Basilichthys Girard, 1854, 258.

Atherinopsis Girard, 1854, 257.

Labidesthes Cope, 1870, 356.
Protistius Cope, 1874, 375.

Atherinops Steindachner, 1875, 381.

Atherinella Steindachner, 1875, 381.

Gastropterus Cope, 1878, 394.

Leuresthes Jordan \& Gilbert, 1880, 403.

Thyrina Jordan \& Culver, 1895, 468.

Tropidostethus Ogilby, 1895, 469.

Lethostole Jordan \& Evermann, 1896, 473.

Eurystole J. \& E., 1896, 473.

Kirtlandia J. \& E., 1896, 473.

Eslopsarum J. \& E., 1896, 472.

Tæniomembras Ogilby, 1898, 483.

Pisciregia Abbott, 1899, 484.

Iso Jordan \& Starks, 1901, 495.

Atherion J. \& S., 1901, 485.

s11 These minute translucent fishes were regarded by Cuvier as the young of Atherima. But Risso describes their egg-laying, and they may be really a distinct form. 
Melaniris Meek, 1902, 500.

Ischnomembras Fowler, 1903, 503.

Atherinomorus Fowler, 1903, 503.

Phoxargyrea Fowler, 1903, 503.

Odontesthes Evermann \& Kendall, 1906, 517.

Xenatherina Regan, 1907, 526.

Craterocephalus McCulloch, 1912, 544.

Pseudothyrina Ribeiro, 1915, 557.

Kronia Ribeiro, 1915, 557.
Colpichthys Hubbs, 1918, 564.

Austromenidia Hubbs, 1918, 564.

Thyrinops Hubbs, 1918, 564.

Hubbesia Jordan, 1919, 566.

Rheocles Jordan \& Hubbs, 1919, 567.

Zanteclites Jordan \& J. Z. Gilbert, 1919, 568.

Archomenidia Jordan \& Hubbs, 1919, 569. Melanorhinus ${ }^{312}$ Metzelaar, 1920.

${ }^{812}$ Metzelaar (Jan), "Over Tropisch Atlantisch Visschen, Amsterdam," 38; type Melanorhinus boeki Metzelaar.

\section{Family 374. 'MUGILID压}

\section{(Mullets)}

Mugil Linnæus, 1758, 15.

Albula Catesby, 1771, 30.

Cestreus Klein, 1777, 43.

Chelon Röse, 1793, 52.

Cephalus (Plumier) Lacépède, 1803, 73.

Agonostomus Bennett, 1830, 174.

Nestis Cuvier \& Valenciennes, 1836, 185.

Dajaus Cuv. \& Val., 1836, 185.

Cestræus Cuv. \& Val., 1836, 185.

Arnion Gistel; 1848, 237.

Joturus Poey, 1860, 299.

Myxus Günther, 1861, 307.
Rhinomugil Gill, 1863, 324.

Chænomugil Gill, 1863, 324.

Gonostomyxus Macdonald, 1869, 355.

Neomy xus Steindachner, 1878, 397.

Querimana Jordan \& Gilbert, 1882, 420.

Eschrichthys Macleay, 1883, 424.

Liza Jordan \& Swain, 1884, 428.

Trachystoma Ogilby, 1887, 438.

Neomugil Vaillant, 1894, 466.

Edalechilus Fowler, 1903, 502.

Squalomugil Ogilby, 1908, 529.

Xenorhynchichthys Regan, 1908, 530.

Family 375. SPHYRENID $\mathbb{E}$

(Barracudas)

Umbla Catesby, 1771, 29.

Sphyrana Klein, 1778, 43.

Sphyræna Röse, 1793, 52.

Sphyræna Bloch \& Schneider, 1801, 58.
Acus (Plumier) Lacépède, 1803, 73.

Spharina ${ }^{313}$ Swainson, 1839.

Agriosphyræna Fowler, 1903, 502.

${ }^{313}$ A misprint for Sphyrcena.

\section{Suborder RHEGNOPTERI}

Family 376. POLYNEMIDE

(Thread-fins)

Polynemus Linnæus, 1758, 15.

Trichidion Klein, 1775, 40.

Polydactylus Lacépède, 1803, 67.

Galeoides Günther, 1860, 297.

Pentanemus Günther, 1860, 296.
Trichidion (Klein) Gill, 1861, 302.

Polistonemus Gill, 1861, 305.

Eleutheronema Bleeker, 1862, 310.

Eleutherochir Bleeker, 1879, 398. 


\section{Series SCOMBRIFORMES}

\section{Family 377. SCOMBRIDA}

(Mackerels)

Scomber Linnæus, 1758, 14.

Pelamys Klein, 1775, 37.

Scomberomorus Lacépède, 1802, 62.

Macrorhynchus (Lacépède) Duméril, $1806,56,75$.

Polipturus Rafinesque, 1815, 89.

Cybium Cuvier, 1829, 129.

Apolectus Bennett, 1831, 173, 175, 284.

Apodontis Bennett, 1831, 173, 284.

Xiphopterus Agassiz, 1835, 181.

Dictyodus Owen, 1839, 196.

Sph yrænodus (Owen) Agassiz, 1841, 209, 219.

Palimphyes Agassiz, 1844, 218, 231.

Isurus (Egerton) Agassiz, 1844, 189, 218.

Gasteroschisma Richardson, 1845, 227.

Acropoma Owen, 1854, 260.

Eucynodus Owen, 1854, 260.

Cordylus Gronow, 1854, 259.

Acanthocybium Gill, 1862, 314.

Lepidocybium Gill, 1862, 314.

Stereodus Owen, 1865, 339.

Scomberodon Van Beneden, 1871, 360.

Chriomitra Lockington, 1879, 400.

Megalolepis Kramberger, 1879, 400.

Pneumatophorus Jordan \& Gilbert, 1882, 420.
Amphodon Storms, 1886, 436.

Lepidothynnus Günther, 1889, 447.

Cerlopoma (Agassiz) Woodward, 1901, 224.

Scombrinus (Agassiz) Woodward, 1901, $224,496$.

Rhonchus (Agassiz) Woodward, 1901, $224,496$.

IsURICH TH Ys Woodward, 1901, 496.

Eoccelopo Ma Woodward, 1901, 496.

SCOMBRAMPHoDon Woodward, 1901, 496.

Chenogaster Lahille, 1903, 505.

Kra mbergeria Simionescu, 1904, 511.

Sierra Fowler, 1905, 512.

Pelam ycybiu m Toula, 1905, 516.

Neocybium Leriche, 1906, 519.

Nesogrammus Evermann \& Seale, 1907, 522.

Rastrelliger Jordan \& Starks, 1908, 528.

Auxides Jordan, 1919, 568.

Tunita J. \& G., 1919, 569.

Turio J. \& G., 1920, 571.

Th yrSOCles J. \& G., 1920, 571.

Thyrsion Jordan, 1920, 571.

ZaPhleges Jordan, 1920, 571.

Ocystias Jordan, 1920, 571.

Xestias ${ }^{314}$ Jordan, 1921.

s14 "Fish Fauna, Cal. Tertiary," 270; type X. iratus Jordan.

\section{Family 378. THUNNID压}

(Tunnies; Albacores)

"The bonitos and tunnies are quite different from other teleostomous fish in having dark red muscles deeply situated on both sides of the vertebrai column."-(K. Kishinouye, Proc. Pan-Pacific Scientific Conference, 1: 229. 1921.)

Thynnus Browne, $1789,48$.

Thynnus Cuvier, 1817, 105.

Orcynus Cuvier, 1817, 105.

Sarda Cuvier, 1829, 129.

Auxis Cuvier, 1829, 129.

Pelamys Cuvier \& Valenciennes, 1831, 573.

\footnotetext{
${ }^{815}$ Misprinted Orycnopsis.

${ }^{818}$ Misprinted Grammatorycnus.
}

Palamita Bonaparte, 1831, 175.

Thunnus South, 1845, 227.

Creotroctes Gistel, 1848, 237.

Orycnus Gill, 1862, 315.

Gymnosarda Gill, 1862, 315 .

Orcynopsis $^{315}$ Gill, 1862, 314.

Grammatorcynus ${ }^{318}$ Gill, 1862, 314. 
Orycnus Gill 1862, 315.

Thynnichthys Giglioli, 1880, 402.

Pelamichthys Giglioli, 1880, 402.

Euthynnus (Lütken) Jordan \& Gilbert, $1882,420$.

Germo Jordan, 1888, 442.

Albacora Jordan, 1888, 442.
Cozlocephalus (Agassiz) Woodward, 1901, 219.

Pralacrus (Ag.) Woodward, 1901, 224.

EOTHYN NUS Woodward, 1901, 496.

Carinicers (Owen) Woodward, (1854) $1901,260,496$.

Ozymandias Jordan, 1919, 569.

\section{Family 379. GEMPYLIDÆ}

(Snake Mackerels; Escolares)

Acinaces Bory de St. Vincent, 1804, 170.

Macrorhynchus (Lacépède) Duméril, $1806,56$.

Thyrsites Cuvier, 1829, 129.

Gempylus Cuvier, 1829, 129 .

Lemnisoma Lesson, 1830, 140. 。

Ruvettus Cocco, 1833, 178.

Acanthoderma Cantraine, 1837, 188.

Aplurus Lowe, 1841, 209.

Prometheus Lowe, 1841, 209.

Epinnula Poey, 1854, 260.

Dicrotus Gumber, 1860, 297.

Thyrsitocepralus Rath, 1859, 292.

Thyrsitops Gill, 1862, 314.
Nesiarchus Johnson, 1862, 319.

Nealotus Johnson, $1865,338$.

Acanthonotus Sauvage, $1870,359$.

Hemith YRSites Sauvage, 1873, 371.

Gyrionemus Vaillant, 1888, 443.

Bathysoma Davis, $1890,450$.

Promethichthys Gill, 1893, 461.

Escolar Jordan \& Evermann, 1896, 468.

Bipinnula Jordan \& Evermann, 1896, 474.

Machærope Ogilby, 1898, 483.

Xenogramma Waite, 1904, 511.

Jordanidia Snyder, 1911, 541.

Rexia Waite, 1911, 541.

\section{Family 380. TRICHIURIDE (Lepidopida)}

(Hair-tails)

Trichiurus Linnæus, 1758, 12.

Gymnogaster Gronow, 1767, 22.

Lepidopus Gonau, 1770, 28.

Enchelyopus Klein, 1775, 57.

Vandellia Shaw, 1803, 73.

Scarcina Rafinesque, 1810, 79.

Xiphotheca ${ }^{817}$ Montagu, 1812, 84.

ANENCHELum Blainville, 1818, 107.

Aphanopus Lowe, 1839, 195.
Zypothyca Swainson, 1839, 200.

LEPIDOPIDES Heckel, 1850, 246.

Lepturus (Artedi) Gill, 1861, 302.

Enchelyopus (Klein) Bleeker, 1862, 310.

Eupleurogrammus Gill, 1862, 314.

Evoxymetopon Poey, 1863, 325.

Trichivrichth Ys Sauvage, 1873, 371.

Benthodesmus Goode \& Bean, 1882, 419.

Lepturacanthus Fowler, 1905, 512.

${ }^{217}$ Written Ziphotheca by Swainson.

\section{Series XIPHIIFORMES}

\section{Family 381. PALAORHYNCHID王}

PALéRHyNCHUM Blainville, 1818, 108.

HemiRhynCHus Agassiz, 1844, 219.
Homalorhy ychus Van Beneden, 1873, 367.

\section{Family 382. BLOCHIID IE}

Blochius Volta, 1796, 54.

Ceroriynchus Agassiz, 1844, 219.

Guiscus Gistel, 1848, 235. 


\section{Family 383. ISTIOPHORID平}

(Sail Fishes; Spear Fishes)

Istiophorus ${ }^{318}$ Lacépède, 1802, 62.

Makaira $^{\text {319 }}$ Lacépède, 1803, 65.

Notistium Herrmann, 1804, 74.

Tetrapturus Rafinesque, 1810, 80.

Skeponopodus Nardo, 1833, 179.
Zanclurus Swainson, 1839, 200.

ENCHEIZIPHIUS Rütimeyer, 1857, 276.

EMBALORHY NCH US Marsh, 1870, 359.

BRACH YRH YNCHUS Van Beneden, 1871, 360.

${ }^{318}$ Written Histiophorus by authors.

"210 Also spelled Machara and Macaria by authors.

Family 384. XIPHIID E

(Sword Fishes)

Xiphias Linnæus, 1758, 12.

ACEstrus (Agassiz) Woodward, 1901,

$221,496$.

\section{Family 385. XIPHIORHYNCHID 2 E}

Xiphiorhynchus Van Beneden, 1871, 360.

Oмmatolampes Fischer von Waldheim, $1850,246$.

\section{Series LUVARIFORMES}

Family 386. LUVARID压

Luvarus Rafinesque, 1810, 79.

Scrofaria Gistel, 1848, 235.

Ausonia Risso, 1826, 120.

\section{Series CORYPHAENIFORMES}

Family 387. CORYPH सENID $\approx$

(Dolphins; Dorados)

Coryphæna Linnæus, 1758, 12.

Hippurus Klein, 1779, 43.

Caranxomorus Lacépède, 1802, 61.

Coryphus (Commerson) Lacépède, 1802, 70.
Lepimphis Rafinesque, 1810, 79.

Lampugus Cuvier \& Valenciennes, 1833, 178.

Sarda Gronow, 1854, 259.

\section{Series BRAMIFORMES}

\section{Family 388. BRAMIDE}

(Sea-breams)

The fishes of this famity have some traits in common with the Selenichthyes, from which they may be derived.

Brama Bloch \& Schneider, 1801, 58.

Lepodus Rafinesque, 1810, 80.

Taractes Lowe, 1843, 215.

Tylometopon (Van Bemmelen) Bleeker, $1876,383$.
Argo Döderlein, 1883, 426.

Collybus Snyder, 1904, 511.

Eumegistus $^{\text {930 }}$ Jordan \& Jordan, 1923.

aso Jordan (David Starr) and Jordan (Eric Knight), "Fishes of Hawaii," Mem. Carnegie Museum, 35; orthotype Eumegistus illustris Jordan \& Jordan. 


\section{Family 389. STEINEGERIID E}

Steinegeria Jordan \& Evermann, 1886, 435.

Family 390. DIANID $\mathbb{E}$

Diana Risso, 1826, 119.

Astrodermus (Bonelli) Cuvier, 1829, 129.

Proctostegus Nardo, 1827, 121.

\section{Family 391. PTERACLID E}

Pteraclis Gronow, 1763, 22.

Pteraclis Gronow, 1772, 22.

Oligopodes (Risso) Cuvier, 1817, 105.

Pteridium Scopoli, 1777, 41.

Oligopus $^{320 z}$ Risso, 1826, 105.

Oligopodus Lacépède, 1800, 57.

Pterycombus Fries, 1837, 189.

Pteraclidus Rafinesque, $1815,88$.

Centropholis Hilgendorf, 1878, 395.

Bentenia Jordan \& Snyder, 1901, 494.

${ }^{8200}$ Type Oligopus niger Risso.

Family 392. ELEPHENORID \&

Elephenor Jordan, 1919, 568.

\section{Series STROMATEIFORMES}

Family 393. STROMATEIDE

Teeth in the gullet; scales small, mostly silvery ; first dorsal more or less rudimentary.

Stromateus Linnæus, 1758, 12.

Rhombus Lacépède, 1800, 56.

Lepterus Rafinesque, 1810, 80.

Fiatola Cuvier, 1817, 106.

Seserinus (Cuvier) Oken, 1817, 106.

Fiatola Risso, 1826, 119.

Peprilus Cuvier, 1829, 129.

\author{
Poronotus Gill, 1861, 302. \\ Psenopsis Gill, 1862, 314. \\ Aspidolepis Geinitz, 1868, 349. \\ Palometa Jordan \& Evermann, 1896, 474. \\ Pterorhombus Fowler, 1906, 517. \\ Eucrotus B. A. Bean, 1912, 541. \\ Toledia Ribeiro, 1915, 557.
}

Family 394. PAMPID E

The fishes of this group are closely similar to the Stromateide in character and appearance, differing, however, in the narrowly restricted gill openings.

Pampus Bonaparte, 1837, 187.

Stromateoides Bleeker, 1851, 248.

Chondroplites Gill, 1862, 314.
Leptolepis (Van Hasselt) Guichenot, 1867, 345.

Pampus (Bonaparte) Fowler, 1905, 513.

\section{Family 395. CENTROLOPHIDE}

This group or family is closely allied to the Stromateida, differing in the continuous dorsal, larger scales, and much more elongate form.

Centrolophus Lacépède, 1803, 64 .

Acentrolophus Nardo, 1827, 121.

Schedophilus Cocco, 1829, 133.
Leirus $^{321}$ Lowe, 1833, 179.

Mupus Cocco, 1833, 178.

Gymnocephalus Cocco, 1838, 191.

${ }^{321}$ Usually written Lirus. 
Pompilus Lowe, 1839, 195.

Palinurus ${ }^{322}$ Dekay, 1842, 288.

Crius Valenciennes, 1844, 223 .

Palinurichthys Bleeker, 1859, 288.

Palinurichthys Gill, 1860, 295.
Pammelas Günther, 1860, 297.

Hoplocoryphis Gill, 1862, 314.

Icichthys Jordan \& Gilbert, 1880, 404.

Ectenias Jordan \& Thompson, 1914, 553.

s2z "N. Y. Fauna Fishes," 118; orthotype P. perciformis Dekay; name preoccupied.
(Omitted in "Genera of Fishes.")

Family 396. NOMEIDE (Psenida)

This group is closely allied to the Stromateida, having, like the latter, teeth in the gullet. It differs in the presence of a well-developed first dorsal fin.

Nomeus Cuvier, 1817, 105.

Psenes Cuvier \& Valenciennes, 1833, 142.

Cubiceps Lowe, 1843, 215.

Seriolella Guichenot, 1848, 238.

Atimostoma Smith, 1849, 244.

Carangodes Heckel, 1856, 270.

Navarchus Filippi \& Verany, 1859, 291.

Neptomenus Günther, 1860, 297.

Trachelocirrus Doumet, 1863, 323.

Bathyseriola Alcock, 1890, 449.

Ariomma ${ }^{323}$ Jordan \& Snyder, 1904, 508.

Hyperoglyphe Günther, 1859, 291.

Icticus $^{224}$ Jordan \& Thompson, 1914, 553.

Probably not distinguishable from Cubiceps.

${ }^{224}$ Icticus, like Icichthys, Schedophilus, and Icosteus, is pelagic, the body soft and limp like a wet rag. Icticus has, however, a distinct spinous dorsal, and Icosteus is covered with tough naked skin beset on the lateral line and fin rays with prickles.

\section{Family 397. TETRAGONURID $\mathbb{E}$}

Allied to the Stromateida, according to Regan, the species having likewise teeth in the gullet. The external characters are, however, very different.

Tetragonurus Risso, 1810, 77. Ctenodax Macleay, 1886, 436.

\section{Series ICOSTEIFORMES}

\section{Family 398. ICOSTEID $\mathbb{E}$}

\section{(Rag Fishes)}

This family and the next seem related to the Centrolophida, but the bones are very feeble and the gullet teeth seem to be wanting.

Icosteus ${ }^{325}$ Lockington, 1880, $404 . \quad$ Schedophilopsis Steindachner, 1881, 417.

${ }^{225}$ In Icosteus the ventral rays, I, 4 ; in Acrotus the fin is entirely wanting.

\section{Family 399. ACROTID王}

Acrotus Bean, 1887, 437.

\section{Series CARANGIFORMES}

Vertebræ strong, 24 to 26 in number. 


\section{Family 400. APOLECTID正}

This group, with the general aspect of Stromateus, has the vertebræ $10+15$ as in Caranx. The species lack the gullet teeth characteristic of the Stromateida. The dorsal and anal are without spines, in the adult at least, and the keeled tail is without scutes. The soft dorsal is inserted farther forward than in Caranx, in front of middle of body.

Apolectus Cuvier \& Valenciennes, 1831, Parastromateus Bleeker, 1865, 336. $174,284$.

\section{Family 401. CARANGID瓜}

\section{(Cavallas)}

These fishes, having much in common with the scombroid fishes, have nevertheless the vertebræ $10+14=24$, as in typical percoids, toward which they form a gradual transition.

Glaucus Klein, 1775, 38.

Saurus Browne, 1789, 48.

Rhomboida Browne, 1789, 48.

Trachurus (Plumier) Lacépède, 1802, 72.

Trachinotus ${ }^{288}$ Lacépède, 1802, 61.

Pelamis (Plumier) Lacépède, 1802, 72.

Cæsiomorus Lacépède, 1802, 61.

Caranx Lacépède, 1802, 60.

Scomberoides Lacépède, 1802, 60 .

Centronotus Lacépède, 1802, 62.

Acanthinion Lacépède, 1803, 64.

Gallus Lacépède, 1803, 64.

Argyreiosus Lacépède, 1803, 64.

Selene Lacépède, 1803, 64.

Guaperza (Plumier) Lacépède, 1803, 72.

Hypacantha ${ }^{\text {277 }}$ Rafinesque, 1810, 80.

Trachurus Rafinesque, 1810, 79.

Tricropterus Rafinesque, 1810, 79.

Hypodis Rafinesque, 1810, 79.

Naucrates Rafinesque, 1810, 80.

Alectis Rafinesque, 1815, 88.

Baillonus Rafinesque, 1815, 89.

Orcynus Rafinesque, 1815, 89.

Seriola Cuvier, 1817, 105.

Citula Cuvier, 1817, 104.

Atropus (Cuvier) Oken, 1817, 105.

Lichia Cuvier, 1817, 105.
Blepharis Cuvier, 1817, 105, 129.

Vomer Cuvier, 1817, 105.

Teratichthys König, 1825, 172.

Micropteryx Agassiz, 1829, 132.

Scyris Cuvier, 1829, 129.

Olistus Cuvier, 1829, 129.

Chorinemus Cuvier \& Valenciennes, $1832,137$.

Pompilus Minding, 1832, 140.

Gallichthys Cuvier \& Valenciennes, 1833, 178.

Porthmeus Cuv. \& Val., 1833, 178.

Nauclerus Cuv. \& Val., 1833, 178.

Hynnis Cuv. \& Val., 1833, 178.

Acanthonemus Agassiz, 1834, 180.

Carangopsis Agassiz, 1835,181, 218.

Platysomus Swainson, 1839, 201.

Zonichthys Swainson, 1839, 200.

Alepes Swainson, 1839, 200.

Elagatis Bennett, 1840, 206.

Paropsis ${ }^{8278}$ Jenyns, 1842.

Ductor Agassiz, 1844, 218.

Pleionemus Agassiz, 1844, 218.

ARCh zus Agassiz, 1844, 218.

Xystophorus Richardson, 1844, 223.

Carangus ${ }^{329}$ Agassiz, 1845.

Selenia ${ }^{220}$ Bonaparte, 1845, 226.

Often corrected to Trachynotus.

227 Misprinted Hypacantus; corrected in appendix to Hypacantha.

Fishes.")

mara "Voyage 'Beagle'," 65; orthotype P. signata Jenyns. (Omitted in "Genera of

${ }^{23}$ This is the first use of Carangus as a generic name. No explanation is given, merely a reference to Cuvier \& Valenciennes. The presumable type is Scomber carangus Bloch.

The name Selenia is preoccupied; Uraspis, with the bucklers hooked forward is a distinct genus. 
Carangoides Bleeker, 1851, 248.

Selar ${ }^{330}$ Bleeker, 1851, 248.

Decapterus Bleeker, 1851, 248.

Gnathanodon Bleeker, 1851, 247.

Leioglossus Bleeker, 1851, 248.

Megalaspis Bleeker, 1851, 248.

Selaroides Bleeker, 1851, 248.

Leptaspis Bleeker, 1852, 250.

Carangichthys Bleeker, 1852, 250.

Seriolichthys Bleeker, 1854, 255.

Thynnus Gronow, 1854, 259.

Trachurus Gronow, 1854, 259.

Vomeropsis, Heckel, 1854, 259.

Uraspis Bleeker, 1855, 264.

Bothrolæmus Holbrook, 1855, 265.

Aipich t hys 'Steindachner, 1859, 298.

Carangus $^{331}$ Girard, 1859, 291.

Doliodon Girard, 1859, 291.

Chloroscombrus Girard, 1859, 291.

ARCH EOIDEs Rath, 1859, 292.

Decaptus Poey, 1861, 309.

Blepharichthys Gill, 1861, 302.

Hemicaranx ${ }^{332}$ Bleeker, 1862.

Carangops Gill, 1862, 316.

Trachurops Gill, 1862, 315.

Gymnepignathus Gill, 1862, 316.

Eustomatodus Gill, 1862, 316.

Evepigymnus Gill, 1862, 316.

Paratractus Gill, 1862, 317.

Halatract Iss Gill, 1862, 317.
Naucratopsis Gill, 1862, 317.

Irex Valenciennes, 1862, 320.

Glaucus (Klein) Bleeker, 1863, 321.

Oligoplites Gill, 1863, 324.

Micropus Kner, 1868, 352.

Pseudovomer Sauvage, 1870, 359.

Desmichtн ys Sauvage, 1877, 392.

Acanthonemopsis Bosniaski, 1878, 393.

Parequula Sauvage, 1880, 406.

Lepidomegas Thominot, 1880, 406.

Hypocaranx Klunzinger, 1884, 429.

Parona Berg, 1895, 466.

Zalocys Jordan \& McGregor, 1898, 482.

Campogramma Regan, 1903, 505.

Rastrum Fowler, 1904, 507.

Rhaphiolepis Fowler, 1905, 512.

Vexillicaranx Fowler, 1905, 512.

Elaphotoxon Fowler, 1905, 512.

Eleria Jordan \& Seale, 1905, 514.

Glaucus (Klein) Fowler, 1906, 517.

Pampanoa Fowler, 1906, 517.

Ulua Jordan \& Snyder, 1908, 528.

Glaucus (Klein) Jordan \& Hubbs, 1917, 562.

Orqueta Jordan, 1919, 567.

LoM POCH ITES Jordan, 1920, 571.

Atule ${ }^{333}$ Jordan, 1923.

Longirostrum ${ }^{234}$ Wakiya, 1923.

Seriolina $^{\text {334a }}$ Wakiya, 1923.

${ }^{330}$ Logotype, by first reviser, Caran $x$ boops, hence equivalent to Trachurops Gill, which name Selar must replace.

as "Carangus Griffith," page 180, "Genera of Fishes," should be suppressed; Carangues is merely quoted as a French name.

283 Versl. Kong. Akad. Wet., 14: 134; orthotype H. marginatus Bleeker. (Omitted in "Genera of Fishes.")

233 "Fishes of Hawaii," Carnegie Mus., in press; orthotype Caranx affinis Rüppell.

${ }^{234}$ Yoshiro Wakiya, "Carangoid Fishes of Japan," Ms., 1923; type Caranx platessa

Cuvier \& Valenciennes.

${ }^{834 a}$ Loc, cit.; orthotype Seriola intermedia Temminck \& Schlege (gill rakers obsolete).

\section{Family 402. NEMATISTIID五}

\section{(Peacock Fishes)}

Nematistius Gill, 1862, 316.

Seriolophus Guichenot, 1867, 345.

\section{Family 403. MENIDE}

Vertebræ $10+15=25$

Mene Lacépède, 1803, 68.

Meneus Rafinesque, 1815, 90.
Gasteronemus Agassiz, 1833, 177.

Gasteracanthus ${ }^{385}$ Agassiz, 1833.

${ }^{235} \mathrm{~A}$ manuscript name, printed but not adopted by Agassiz, being preoccupied; replaced by Gastronemus. Poissons Fossiles, 5: 20; orthotype G. rhomboidalis Agassiz ms. 


\section{Family 404. POMATOMIDE}

(Blue Fishes)

Saltatrix Catesby, 1771, 30.

Pomatomus Lacépède, 1803, 63.

Gonenion Rafinesque, 1810, 80.

Lopharis Rafinesque, 1810, 80.

Temnodon Cuvier, 1817, 106.
Sypterus Eichwald, 1841, 208.

Chromis Gronow, 1854, 259.

Sparactodon Rochebrune, 1880, 405.

LophaR Jordan \& Gilbert, 1919, 569.

\section{Family 405. RACHYCENTRID\&E}

(Sergeant Fishes)

Rachycentron ${ }^{336}$ Kaup, 1826, 121.

Spinax (Commerson), Cuvier \& Valęnciennes, 1831, 175.
Elacate Cuvier \& Valenciennes, 1831, 129.

Meladerma Swainson, 1839, 200.

${ }^{236}$ Name later corrected to Rachycentrum.

\section{Family 406. LACTARIID王}

Lactarius Cuvier \& Valenciennes, 1833, Platylepes Swainson, 1839, 200. 178.

\section{Family 407. LEIOGNATHID在 ${ }^{337}$}

Leiognathus Lacépède, 1803, 64.

Halex Commerson, 1803, 71.

Equula Cuvier, 1817, 105.

Gazza Rüppell, 1835, 184.

Argyrlepes Swainson, 1839, 200.
Secutor Gistel, 1848, 235.

Deveximentum Fowler, 1904, 507.

Eubleekeria Fowler, 1904, 507.

Equulites Fowler, 1904, 507.

Aurigequula ${ }^{338}$ Fowler, 1918.

${ }^{287}$ The resemblance of Leiognathus to Gerres seems to be superficial, not indicating any special affinity.

${ }^{\$ 3}$ Proc. Acad. Nat. Sci. Phila., 70: 17; orthotype Leiognathus fasciatus Fowler.

\section{Family 408. BATHYCLUPEID压}

A deep-water group of uncertain affinities.

Bathyclupea Alcock, 1891, 454.

\section{Series KURTIFORMES}

Family 409. KURTID $\mathbb{E}$

A single genus of uncertain relationships.

Kurtus ${ }^{239}$ Bloch, 1786, 44.

${ }^{330}$ Corrected to Cyrtus and Curtus by authors.

\section{Family 410. PERCID蚱}

\section{(Perch)}

Perca Linnæus, 1758, 13.

Cernua Schäfer, 1761, 16.

Schraitzer Schäfer, 1761, 16.

Asperulus Schäfer, 1761, 16.

Asper Schäfer, 1761, 16.
Acerina Güldenstädt, 1774, 32.

Percis Klein, 1775, 39.

Asperulus Klein, 1775, 40.

Gymnocephalus Bloch, 1793, 51.

Cephimmus Rafinesque, $: 815,89$. 
Zingel (Cuvier) Oken, 1817, 104.

Sander (Cuvier) Oken, 1817, 104.

Acerina Cuvier, 1817, 104, 125.

Pogostoma ${ }^{840}$ Rafinesque, 1818, 108.

Aplocentrus ${ }^{340}$ Rafinesque, $1819,110$.

Leucops ${ }^{8+0}$ Rafinesque, 1819, 110.

Pomacampsis ${ }^{3+0}$ Rafinesque, 1820, 111.

Stizostedion $^{841}$ Rafinesque, 1820, 111.

Sandat Cloquet, 1827, 122.

Cernua Fleming, 1828, 123.

Sandrus Stark, 1828, 123.

Cingla Stark, 1828, 123.

Lucioperca Cuvier \& Valenciennes, 1828, 124.

Aspro Cuv. \& Val., 1828, 124.

Sandat Bory de St. Vincent, 1828, 124.

Acerina Cuvier, 1820, 129.

Schilus Krynicki, 1832, 176.

Podocys Agassiz, 1838, 191.

Percarina Nordmann, 1840, 207.
Pach ygaster Giebel, 1847, 231.

Peroptera Gistel, 1848, 237.

Gremilla Gistel, 1848, 234.

Sa NDRoserrus Gervais, 1852, 251.

Asperulus (Klein) Gill, 1861, 303.

Leptoperca Gill, 1861, 306.

Cynoperca Gill \& Jordan, 1877, 390.

Mimoperca Gill \& Jordan, 1877, 390.

Mroplosus Cope, 1877, 388.

Epitrachys Schulze, 1889, 448.

Podocephalus (Agassiz) Woodward, (1845) 1901, 224.

BRACH YGNATHUS (Agassiz) Woodward, (1845) 1901, 224.

Caloperca (Agassiz) Woodward, (1845) 1901, 224.

Percostoma (Agassiz) Woodward, (1845) $1901,224$.

Cristigorina Leriche, 1905, 515.

EOPERCA Jordan, 1919, 568.

${ }^{840}$ All these genera of Rafinesque appear to be mythical, an opinion derived from drawings in his private notebook preserved in the Smithsonian Institution.

${ }^{341}$ Name corrected to Stizostethium.

\section{Family 411. ETHEOSTOMID正}

\section{(Darters; Crawl-a-bottoms)}

These dwarf or rather concentrated perches are peculiar to the waters of the eastern United States. They differ from the Percidce in having six branchinstegals instead of seven, the head unarmed and the air-bladder obsolete or nearly so. Anal spines two, rarely one.

Etheostoma Rafinesque, 1819, 110.

Diplesion Rafinesque, 1820, 111.

Percina Haldeman, 1842, 211.

Pileoma Dekay, 1842, 210.

Boleosoma Dekay, 1842, 210.

Pœcilosoma Agassiz, 1850, 245.

Hadropterus Aggasiz, 1854, 254.

Pœcilichthys Agassiz, 1854, 254.

Catonotus Agassiz, 1854, 254.

Hyostoma Agassiz, 1854, 254.

Oligocephalus Girard, 1859, 290.

Arlina Girard, 1859, 290.

Estrella Girard, 1859, 290.

Boleichthys Girard, 1859, 290.

Alvordius Girard, 1859, 290.

Alvarius Girard, 1859, 290.
Pleurolepis (Agassiz) Putnam, 1863, 328.

Microperca Putnam, 1863, 328.

Hololepis ${ }^{\text {412 }}$ (Agassiz) Putnam, 1863.

Nothonotus (Agassiz) Putnam, 1863, 328.

Cottogaster Putnam, 1863, 328.

Asproperca Heckel, 1860, 295.

Hypohomus Cope, 1870, 355.

Astatichthys Vaillant, 1873, 371.

Plesioperca Vaillant, 1873, 371.

Imostoma Jordan, 1877, 390 .

Ammocrypta Jordan, 1877, 390.

Nanostoma (Putnam) Jordan, 1877, 390.

Ericosma Jordan \& Copeland, 1877, 390.'•

Rheocrypta Jordan, 1877, 390.

Vaillantia Jordan, 1878, 395.

Ioa Jordan \& Brayton, 1878, 395.

${ }^{241 \mathrm{a}}$ Omitted in "Genera of Fishes"; type Boleosoma barratti Holbrook (near Boleichthys or identical with it). 
Ulocentra Jordan, 1878, 395.

Serraria Gilbert, 1884, 428.

Crystallaria Jordan \& Gilbert, 1885, 432.

Rhothøeca Jordan, 1885, 432.

Copelandellus Jordan \& Evermann, 1896, 474.

Psychromaster J. \& E., 1896, 474.
Claricola J. \& E., 1896, 474

Nivicola Jordan, 1896, 474.

Rafinesquiellus Jordan \& Evermann, 1896, 474.

Torrentaria J. \& E., 1896, 474.

Swainia Jordan \& Evermann, 1896, 474.

Vigil Jordan, 1919, 567.

\section{Family 412. PERCICHTHYID}

This family and the next are confined to rivers of the Chilean region. They are intermediate between Percide and Moronida.

Percichthys Girard, 1854, 258.

Percosoma Gill, 1861, 304.

Deuteropterus Gill, 1861, 304.

\section{- Family 413. PERCILIID EE}

Percilia Girard, 1854, 258.

\section{Family 414. APOGONID王 (Amiide; Chilodipteride) (Cardinal Fishes)}

Anal spines two, rarely one; vent normal in position.

Amia Gronow, 1763, 20.

Apogon Lacépède, 1802, 62.

Cheilodipterus ${ }^{342}$ Lacépède, 1802, 63.

Aspro (Commerson) Lacépède, 1803, 70.

Ostorhinchus Lacépède, 1803, 63.

Dipterodon Lacépède, 1803, 63.

Epigonus Rafinesque, 1810, 83 .

Clodipterus Rafinesque, 1815, 89.

Macrolepis Rafinesque, 1815, 89.

Pomatomus (Risso) Cuvier \& Valenciennes, 1828, 124.

Apogonoides Bleeker, 1849, 240.

Microichthys Rüppell, 1852, 251.

Amia Gronow, 1854, 259.

Apogonichthys Bleeker, 1854, 256.

Monoprion Poey, 1860, 298.

Glossamia Gill, 1863, 323.

Lepidamia Gill, 1863, 323.

Archamia Gill, 1863, 323.

Paramia Bleeker, 1863, 322.

Pseudamia Bleeker, 1865, 336.

Mionorus Krefft, 1867, 346.

Pristiapogon Klunzinger, 1870, 358.

Dinolestes $^{3+3}$ Klunzinger, 1872, 366.
Lanioperca ${ }^{243}$ Günther, 1872, 365.

Neosphyræna ${ }^{343}$ Castelnau, 1872, 362.

Vincentia Castelnau, 1872, 363.

Percamia Bleeker, 1876, 382.

Telescops Bleeker, 1876, 382.

Gulliveria Castelnau, 1878, 393.

Pomatomichthys Giglioli, 1880, 402.

Mónosira Poey, 1881, 417.

Melanostoma Steindachner \& Döderlein, $1883,426$.

Synagrops Günther, 1887, 437.

Telescopias Jordan \& Snyder, 1901, 495.

Fowleria Jordan \& Evermann, 1903, 504.

Galeagra Heller \& Snodgrass, 1903, 503.

Foa Jordan \& Seale, 1905, 514.

Hynnodus Gilbert, 1905, 513.

Gymnapogon Regan, 1905, 515.

Astrapogon Fowler, 1907, 523.

Siphamia Weber, 1909, 534.

Rhabdamia Weber, 1909, 534.

Neamia Smith \& Radcliffe, 1912, 544.

Amioides Smith \& Radcliffe, 1912, 544.

Nectamia Jordan, 1917, 562.

Xystramia Jordan, 1917, 562.

${ }^{342}$ Written Chilodipterus by authors.

${ }^{343}$ These three generic names refer to the same fish, Dinolestes muelleri, of Australia. 
Zoramia Jordan, 1917, 562.

ERITima Jordan \& Gilbert, 1919, 569.

Adenapogon ${ }^{344}$ McCulloch, 1921.

Brephamia ${ }^{345}$ Jordan, 1922.
Scombrolabrax ${ }^{347}$ Roule, 1922.

Maccullochina ${ }^{\text {34t }}$ Jordan, 1923.

Scepterias ${ }^{848}$ Jordan \& Jordan, 1923.

${ }^{84}$ Records Austral. Mus., 13: 132; orthotype Apogon roseigaster Ramsay \& Ogilby.

sus "Fishes of Hawaii," Mem. Carnegie Museum, 43; orthotype Amia parvula Smith \& Radcliffe.

${ }^{318}$ Loc. cit. 44 ; orthotype Scombrops serratospinosa Smith \& Radcliffe.

${ }^{347}$ Roule, Bull. Sci. Oceanogr., No. 408, March 23, 1922; type S. heterolepis Roule; a deep-sea fish from Madeira. Spinous dorsal deeply divided; anal spine single; scales cycloid, very irregular as to size.

${ }^{848} \mathrm{Op}$. cit. 44 ; orthotype $S$. fragilis Jordan \& Jordan, from Hawaii.

\section{Family 415. SCOMBROPID㤅}

Allied to the Apogonidce, but with three or four anal spines. The number in the Apogonida, as in the Percida and Etheostomatide, is but two or rarely one.

Scombrops Temminck \& Schlegel, 1845, 227.

Sphyrænops Poey, 1860, 299.

Latebrus Poey, 1860, 298.

Amiichthys Poey, 1886, 435.

Hypoclydonia Goode \& Bean, 1895, 468.

Apogonops Ogilby, 1896, 475.

Parasphyrænops Bean, 1912, 542.

Neoscombrops Gilchrist, 1922. (See Index.)

\section{Family 416. ACROPOMATIDE}

Three anal spines; vent anterior.

Acropoma Temminck \& Schlegel, 1843.

216.

Parascombrops ${ }^{399}$ Alcock, 1890, 450.

Oxyodon Brauer, 1906, 517.

Brephostcma Alcock, 1889, 444.

${ }^{849} \mathrm{~A}$ synonym of Acropoma.

\section{Family 417. AMBASSIDÆ}

Chanda Hamilton, 1822, 114.

Ambassis (Commerson) Cuvier \& Valenciennes, 1828, 124.

Priopis (Kuhl \& Van Hasselt) Cuvier \& Valenciennes, 1830, 174.

Hamiltonia Swainson, 1839, 200.

Bogoda Bleeker, 1853, 253, 275.

Parambassis Bleeker, 1874, 374.

Pseudambassis Bleeker, 1876, 383.

Acanthoperca Castelnau, 1878, 393.

Pseudoambassis Castelnau, 1878, 393.

Tetracentrum Macleay, 1883, 425.

Family 418. CENTRARCHID五 (Eucentrarchide; Micropterida) (Black Bass; Sun Fishes; Pumpkin-seeds)

The name Centrarchus of Cuvier was framed for those sun fishes which had more than three anal spines. The first species mentioned (eneus) is an Ambloplites, and this, it appears, was the only one actually seen by the author. But he also includes, without question, the Labrus irideus and Labrus macropterus of Lacépède, irideus being formally indicated as type of Centrarchus by Agassiz in 1854, by Holbrook in 1860, and by Jordan \& 
Gilbert in 1882. There seems no reason for changing this arrangement as with Culver irideus was not a "species inquirenda."

Micropterus Lacépède, 1803, 63.

Pomoxis Rafinesque, 1818, 109.

Calliurus Rafinesque, 1819,110.

Lepomis $^{350}$ Rafinesque, 1819, 110.

Pomotis Rafinesque, 1819, 110.

Apomotis Rafinesque, 1819, 110.

Aplesion Rafinesque, 1820, 111.

Ichthelis $^{351}$ Rafinesque, 1820.

Dioplites Rafinesque, 1820, 111.

Nemocampsis Rafinesque, 1820, 111.

Ambloplites Rafinesque, 1820, 111.

Aplites Rafinesque, 1820, 111.

Telipomis Rafinesque, 1820, 111.

Huro Cuvier \& Valencienines, 1828, 124.

Centrarchus Cuvier, 1829, 126.

Pomotis Cuvier, 1829, 126.

Gristes $^{352}$ Cuvier, 1829, 126.

Bryttus Cuvier \& Valenciennes, 1831, 137.
Archoplites Gill, 1861, 305.

Mesogonistius Gill, 1864, 332.

Chænobryttus Gill, 1864, 332.

Hyperistius Gill, 1864, 332.

Eucentrarchus Gill, 1864, 332.

Acantharchus Gill, 1864, 332.

Enneacanthus Gill, 1864, 332.

Hemioplites Cope, 1867, 344.

Glossoplites $^{883}$ Jordan, 1876.

Helioperca Jordan, 1877, 390.

Xystroplites Jordan, 1877, 388.

Erichæta Jordan, 1877, 391.

Eupomotis Gill \& Jordan, 1877, 389.

Xenotis Jordan, $1877,390$.

Copelandia Jordan, 1877, 390.

Plioplarchus Cope, 1883, 422.

Oligoplarchus Cope, 1891, 454.

Centrarchites Cockerell, 1919, 566.

${ }^{850}$ Later corrected to Lepiopomus, an inacceptable alteration.

${ }^{351}$ Ichthyologia Ohiensis, 27; type Labrus auritus Linnæus; same as Lepomis.

(Omitted in "Genera of Fishes.")

${ }^{352}$ Later written Grystes.

353 "Manual Vertebrates," Ed. 1, 223; type Calliurus melanops Girard; same as Chanobryttus Gill. (Omitted in "Genera of Fishes.")

\section{Family 419. ELASSOMID五}

(Dwarf Sun Fishes)

Elassoma Jordan, 1877, 390.

\section{Family 420. KUHLIIDE}

\section{(Mountain Bass; Sesele)}

Kuhlia Gill, $1861,304$.

Moronopsis Gill, 1863, 324.

Nannoperca Günther, 1862, 307.

Paradules Bleeker, 1863, 322.

Paradules Klunzinger, 1872, 366.

Edelia Castelnau, 1873, 368.

Microperca Castelnau, 1873, 368.
Platysoma ${ }^{854}$ (Liénard) Scudder, (1832) 1882.

Herops De Vis, 1885, 431.

Boulengerina Fowler, 1904, 507, 523.

Safole Jordan, 1912, 543.

Parakuhlia Pellegrin, 1913, 549.

8st Platysoma Liénard (as "Platysome"), Proc. Zool Soc. Lond., 1832, II, 112; type Dules caudavittatus Cuvier \& Valenciennes; latinized by Scudder 1882; name preoccupied; same as Safole Jordan.

\section{Family 421. CENTROPOMID E}

(Robalos)

Macrocephalus Browne, $1789,47$.

Centropomus Lacépède, 1803, 63.

Oxylabrax Bleeker, 1876, 382.
Macrocephalus (Browne) Bleeker, 1876, 383.

Platycephalus Ribeiro, 1902, 500. 


\section{Family 422. LATID死}

This group and the next two are usually united with the Serranide (including Epinephelide), forming thus a vast and divergent. family, which for our purpose may be conveniently separated into some of its component parts. These are usually and perhaps justly regarded as subfamilies. Boulenger places Lates with the Centropomida and Regan adds the Ambassida also.

Lates Cuvier \& Valenciennes, 1828, $124 . \quad$ Paralates Sauvage, 1883, 425.

Psammoperca Richardson, 1844, 223. Platylates Storms, 1887, 439.

Cnidon Müller \& Troschel, 1849, 243. Pseudolates Priem, 1898, 483.

Hypopterus Gill, 1861, 304.

Prolates Priem, 1899, 487.

Pseudolates Alleyne \& Macleay, 1877, 387. Luciolates Boulenger, 1914, 552.

\section{Family 423. MORONID王}

(White Bass)

Maxillary without supplemental bone. The fossil forms referred to this group and the preceding can not be placed with accuracy.

\section{Labrax Klein, 1775, 39 .}

Morone Mitchill, 1814, 86.

Roccus Mitchill, 1814, 86.

Lepibema Rafinesque, 1820, 111.

Labrax (Klein) Cuvier, 1829.

S merdis Agassiz, 1833, 177.

Cyclopoma Agassiz, 1833, 177.
Dapalis Gistel, 1848, 237.

Platacanthus Fischer von Waldheim, $1850,246$.

Dicentrarchus Gill, 1860, 296.

Percalates Ramsay \& Ogilby, 1887, 439.

Chrysoperca Fowler, 1907, 523.

\section{Family 424. OLIGORID㤅}

Maxillary with a supplemental bone, as in Epinephelus. This group is very close to the Moronida, both being usually merged in the Serranida.

Perca-Labrax Temminck'\& Schlegel, $1842,213$.

Lateobrax Bleeker, 1857, 274.

Percalabrax (Schlegel) Günther, 1859, 291.
Oligorus Günther, 1859, 291.

Odontolabrax Bleeker, 1873, 367.

Homodemus De Vis, 1885, 431.

Malakichthys Döderlein, 1883, 426.

Satsuma Smith \& Pope, 1906, 520.

\section{Family 425. NIPHONID压}

Niphon ${ }^{355}$ Cuvier \& Valenciennes, 1828.

${ }^{855}$ Cuvier \& Valenciennes, Hist. Poss., 2: 131; orthotype Niphon spinosus Cuvier \& Valenciennes. (Omitted in "Genera of Fishes.")

\section{Family 426. EPINEPHELID不}

(Sea Bass; Groupers; Garrupas)

The great group of genera commonly called percoid (although centering rather around Epinephelus, Perca being a somewhat aberrant form) is here provisionally divided into several families obviously of very unequal value. Several of them usually and very properly, no doubt, given the rank 
of subfamily. Even after the removal of various outlying genera, the Epinephelida are very much diversified and further subdivision is natural. Whatever rank the divisions may receive, the presence of supplemental maxillary by which character the Epinephelidce are set off from the Serranida is a matter of importance, perhaps adequate to define a separate family, although in some groups, as the Centrarchide, it disappears by degrees.

Cugupuguacu Catesby, 1771, 30.

Daba Forskål, 1775, 34.

Louti Forskål, 1775, 34.

Bodianus $^{388}$ Block, 1790, 49.

Epinephelus Bloch, 1793, 51.

Grammistes Bloch \& Schneider, 1801, 58.

Cephalopholis B. \& S., 1801, 59.

Alphestes B. \& S., 1801, 59.

Chrysomelanus (Plumier) Lacépède, 1803, 72.

Notognidion Rafinesque, $1810,80$.

Polyprion (Cuvier) Oken, 1817, 104, 117.

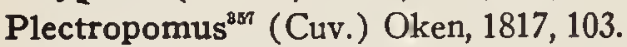

Rypticus ${ }^{388}$ Cuvier, 1829, 125.

Macquaria Cuvier \& Valenciennes, 1830, 135.

Merou Bonaparte, 1831, 175.

Cerna Bonaparte, 1837, 141, 187.

Uriphaëton Swainson, 1839, 198.

Variola Swainson, 1839, 198.

Cynichthys Swainson, 1839, 198.

Cromileptes Swainson, 1839, 198.

Centrogenys Richardson, 1842, 212.

Aulacocephalus Temminck \& Schlegel, $1842,213$.

Myriodon Brisout de Barneville, 1847, 230.

Paschalestes Gistel, 1848, 236.

Serranichthys Bleeker, 1855, 264.

Smecticus Valenciennes, 1855, 266.

Stereolepis Ayres, 1858, 277.

Trachypoma Günther, 1859, 291.

Pogonoperca Günther, 1859, 291.

Anyperodon Günther, 1859, 291.

Promicropterus Gill, 1861, 302.

Liopropoma Gill, 1861, 304.

Dermatolepis Gill, 1861, 304.

Prospinus Poey, 1861, 308.

Siniperca Gill, 1862, 313.
Lioperca Gill, 1862, 315.

Schistorus Gill, 1862, 315.

Gonioplectrus Gill, 1862, 315.

Plectroplites Gill, 1862, 315 .

Acanthistius Gill, 1862, 315.

Labroperca Gill, 1862, 315.

Mycteroperca Gill, 1862, 315.

Hyporthodus Gill, 1862, 304.

Chorististium Gill, 1862, 313.

Petrometopon Gill, 1865, 337.

Enneacentrus Gill, 1865, 337.

Trisotropis Gill, $1865,337$.

Plectroperca Peters, 1865, 339.

Priacanthichthys Day, 1868, 349.

Promicrops Poey, 1868, 353.

Eleutheractis Cope, 1871, 361.

Ctenolates Günther, 1871, 361.

Menephorus Poey, 1871, 362.

Murrayia Castelnau, 1872, 362.

Riverina Castelnau, 1872, 362.

Actenolepis Dybowski, 1872, 364.

Bostockia Castelnau, 1873, 368.

Odontolabrax Bleeker, 1873, 367.

Hectoria Castelnau, 1873, 368.

Pikea Steindachner, 1874, 377.

Phaëthonichthys Bleeker, 1876, 382.

Hemilutjanus Bleeker, 1876, 382.

Homalogrystes Alleynes \& Macleay, $1877,387$.

Itaiara Vaillant \& Bocourt, 1878, 397.

Megaperca Hilgendorf, 1878, 395.

Doderleinia Steindachner, 1883, 426.

Acanthocephalus Döderlein, 1883, 426.

Labracopsis Steindachner \& Döderlein, $1883,426$.

Garrupa Jordan \& Eigenmann, 1890, 452.

Dinoperca Boulenger, 1895, 466.

Pseudalphestes Boulenger, 1895, 466.

${ }^{260}$ Bodianus belongs here by first restriction, not by tautonomy, as Bodianus bodianus is a labroid fish.

${ }^{257}$ Later written Plectropoma.

${ }^{208}$ Later corrected to Rhypticus. 
Pomodon Boulenger, 1895, 466.

Xystroperca Jordan \& Evermann, 1896, 474.

Archoperca J. \& E., 1896, 474.

Enneistus J. \& E., 1896, 474.

Coreoperca Herzenstein, 1896, 472.

Centristhmus Garman, 1899, 486.

Epinephelides Ogílby, 1899, 487.

Bryttosus Jordan \& Snyder, 1900, 490.

Eteliscus J. \& S., 1900, 490.
Corusculus J. \& E., 1901, 494.

Gilbertella Waite, 1902, 501.

Ethaloperca Fowler, 1904, 507.

Gennadius Jordan \& Seale, 1907, 525.

Epelytes Evermann \& Radcliffe, 1917, 562.

Mustelichthys Tanaka, 1918, 565.

EMMACH ERE Jordan \& J. Z. Gilbert, 1919, 569.

Rhabdosebastes ${ }^{359}$ Fowler \& Bean, 1922.

${ }^{250}$ Fowler \& Bean, "Fishes from Formosa and the Philippine Islands," Proc. U. S, Nat. Mus., 62: 60; orthotype Sebastes stolicza Day; same as Gennadius Jordan \& Seale, both being synonyms of Centrogenys. This highly aberrant form with the lower pharyngeals united, bears a deceptive resemblance to species of Sebastes.

\section{Family 427. SERRANID正 \\ (Sea Bass)}

Supplemental maxillary wanting.

Anthias Bloch, 1792, 50.

Hepatus Röse, 1793, 52.

Aylopon Rafinesque, 1810, 80.

Serranus Cuvier, 1817, 103.

Dules Cuvier, 1828, 126.

Centropristes ${ }^{800}$ Cuvier, $1829,126$.

Dulichthys Bonaparte, 1831, 175.

Acanus Agassiz, 1838,191.

Callanthias Lowe, 1839, 195.

Prionodes Jenyns, 1842, 211.

Caprodon 'Temminck \& Schlegel, 1843, 216.

Diplectrum Holbrook, 1855, 265.

Paralabrax Girard, 1856, 269.

Luspia Costa, 1858, 574.

Atractoperca Gill, 1861, 305.

Triloburus Gill, 1861, 302.

Brachyrhinus Gill, 1862, 315.

Hypoplectrus Gill, 1862, 315.

Hypoplectrodes Gill, 1862, 315 .

Mentiperca Gill, 1862, 314.

Haliperca Gill, 1862, 315.

Gonioperca Gill, 1862, 315 .

Pronotogrammus Gill, 1863, 323.

Paranthias Guichenot, 1868, 352.

Holanthias Günther, 1869, 351.

Paracentropristes Klunzinger, 1870, 358 .

Pseudoserranus Klunzinger, 1870, 358.

Cæsioperca Castelnau, 1872, 375.
Pseudanthias Bleeker, 1873, 367.

Plectranthias Bleeker, 1873, 367.

Dactylanthias Bleeker, 1873, 367.

Hemianthias Steindachner, 1874, 377.

Neoanthias Castelnau, 1878, 393.

Symphysanodon Bleeker, 1878, 393.

Cratinus Steindachner, 1878, 397.

Colpognathus Klunzinger, 1880, 575.

Bathyanthias Günther, 1880, 403.

Properca Sauvage, 1880, 405.

Creolus Jordan \& Gilbert, 1882, 420.

Hyposerranus Klunzinger, 1884, 429.

Neoniphon De Vis, 1885, 431.

Gilbertia ${ }^{361}$ Jordan \& Eigenmann, 1890, 452.

Serranellus J. \& E., 1890, 452.

Chelidoperca Boulenger, 1895, 466.

Ocyanthias Jordan \& Evermann, 1896, 474.

Anagramma Ogilby, 1899, 437.

Grammatonotus Gilbert, 1905, 513.

Sayonara Jordan \& Seale, 1906, 518.

Eudulus Fowler, 1907, 523.

Callidulus Fowler, 1907, 523.

Xenanthias Regan, 1908, 529

Zalanthias Jordan \& Richardson, 1910, 536.

Sacura Jordan \& Richardson, 1910, 536.

${ }^{300}$ Later written Centropristis.

${ }^{861}$ This genus is probably distinct from Hypoplectrodes. 
Sphenanthias Weber, 1913, 551.

Pteranthias Weber, 1913, 551.

Lepidoperca Regan, 1914, 554.

Serranops Regan, 1914, 554.

Franzia Jordan \& Thompson, 1914, 554.

Rosanthias Tanaka, 1917, 563.
Selenanthias Tanaka, 1918, 565 .

Leptanthias Tanaka, 1918, 565.

Rhyacanthias ${ }^{362}$ Jordan, 1921.

Percanthia s ${ }^{383}$ Tanaka, 1922.

Parosphenanthias Gilchrist, 1922. (See Index.)

${ }^{3 e 2}$ Jordan, U.S. Nat. Mus., $59: 547$; orthotype R. carlsmithi Jordan.

${ }^{303}$ Tanaka, Fishes Japan, 32: 591; orthotype Callanthias japonicus Franz.

\section{Family 428. PLESIOPID $\approx$}

This family and the next three are closely related, and also allied to the Anthiince among the Serranide.

Plesiops (Cuvier) Oken, 1817, 102.

Pharopteryx Rüppell, 1828, 122, 251.

Trachinops ${ }^{36 t}$ Günther, 1861.

Ruppelia Castelnau, $1873,367$.

Bleeckeria Castelnau, 1873, 368.
Paraplesiops Bleeker, 1875, 378, 383.

Cirrhiptera (Kuhl \& Van Hasselt)

Bleeker, 1876, 383.

Tosana Smith \& Pope, 1906, 520.

Belonepterygion McCulloch, 1915, 556.

${ }^{804}$ Günther, Cat. Fish, 3: 366; type Trachinops taniatus Günther. (Omitted in "Genera of Fishes.")

\section{Family 429. ACANTHOCLINID无}

Acanthoclinus Jenyns, 1842, 212.

Acanthoplesiops Regan, 1912, 545.

\section{Family 430. PSEUDOPLESIOPID $\mathbb{E}$}

Pseudoplesiops Bleeker, 1858, 278.

\section{Family 431. PSEUDOCHROMID瓜}

Pseudochromis Rüppell, 1835, 184.

Labristoma Swainson, 1839, 200.

Cichlops Müller \& Troschel, 1858, 278.

Gramma Poey, 1868, 353.

Dampieria Castelnau, 1875, 378.

Pseudogramma Bleeker, 1875, 378.
Leptochromis Bleeker, "1876, 382.

Labracinus (Schlegel) Bleeker, 1876, 383.

Stigmatonotus Peters, 1876,386.

Onar De Vis, $1885,431$.

Nesiotes De Vis, 1884, 427.

Nematochromis Weber, 1913, 551.

Rhegma Gilbert, 1900, 490.

\section{Family 432. RHEGMATIDE}

\section{Family 433. PRIACANTHID $\mathbb{E}$}

(Big-eyes)

Abuhamrur Forskål, 1771, 34 .

Priacanthus Cuvier, 1817, 104.

Boops Gronow, 1854, 258.
Bogota Blyth, 1860, 294, 383.

Pseudopriacanthus Bleeker, 1869, 353.

\section{Family 434. PEMPHERID}

(Catalufas) fishes.

A group of uncertain relationships, usually placed near the scombroid

Pempheris Cuvier, 1829, 128.

Parapriacanthus Steindachner, 1870, 360.

Pempherichthys Klunzinger, 1871, 361.

Leptobrama Steindachner, 1878, 397.
Neopempheris Macleay, 1881, 416.

Priacanthopsis Fowler, 1906, 517.

Catalufa Snyder, 1911, 541.

Liopempheris Ogilby, 1913, 549. 


\section{Family 435. DIPLOPRIONID $\approx$}

Diploprion (Kuhl \& Van Hasselt) Cuvier

\& Valenciennes, 1828, 124.

\section{Family 436. LOBOTIDE}

\section{(Flashers)}

Coius Hamilton, 1822, 172.

Datnioides Bleeker, 1852, 252.

Lobotes Cuvier, 1829, 128.

Verrugato $^{364 a}$ Jordan, 1923.

${ }^{364 a} V$ errugato Jordan, 1924; new generic name; type Lobotes pacificus Gilbert; distinguished by the feebleness of head serrations.

\section{Family 437. GLAUCOSOMID $\mathbb{E}$}

Glaucosoma Temminck \& Schlegel, 1843, 216.

Breviperca ${ }^{385}$ Castelnau, 1873, 378.

Reganichthys Ogilby, 1915, 557.

${ }^{\text {ses }}$ Young of Glaucosoma.

\section{Family 438. ARRIPIDE}

Arripis Jenyns, 1842, 211.

Homod on Brisout de Barneville, 1847, 230.

\section{Family 439. XENICHTHYID压}

These genera are placed by Regan among the Pomadasida.

Xenichthys Gill, 1863, 324.

Xenocys Jordan \& Bollman, 1889, 447.

Xenistius Jordan \& Gilbert, 1882, 420.

\section{Family 440. HOPLOPAGRID $\approx$}

Hoplopagrus Gill, 1861, 304

\section{Family 441. LUTIANIDE}

(Snappers)

To this family Regan refers the allies of Casio, together with Aphareus, Propoma, and Hoplopagrus.

Salpa Catesby, 1771, 30.

Turdus Catesby, 1771, 30.

Naqua Forskål, 1771, 34.

Anthea Catesby, 1771, 31.

Hobar Forskål, 1775, 34.

Lutianus $^{386}$ Bloch, 1790, 49.

Scirnus (Commerson) Lacépède, 1802, 69.

Sarda (Plumier) Lacépède, 1802, 72.

Pagrus (Plumier) Lacépède, 1803, 72.

Sargus Plumier, 1803, 72.

Diacope Cuvier, 1815, 94.

Mesoprion Cuvier \& Valenciennes, 1828, 124.
Etelis Cuv. \& Val., 1828, 124.

Aprion Cuv. \& Val., 1830, 135.

Apsilus Cuv. \& Val., 1830, 135.

Elastoma Swainson, 1839, 198.

Chætopterus Temminck \& Schlegel, 1844, 223.

Genyoroge Cantor, 1849, 241.

Pristipomoides Bleeker, 1852, 250.

Macrops Duméril, 1856, 269.

Neomænis Girard, 1859, 291.

Macolor Bleeker, 1860, 294.

Ocyurus Gill, 1862, 315.

Proamblys Gill, 1862, 315.

\footnotetext{
${ }^{306}$ Also spelled Lutjanus.
} 
Rhomboplites Gill, 1862, 315.

Platyinius ${ }^{307}$ Gill, 1862, 315 .

Hypoplites Gill, 1862, 315 .

Evoplites Gill, 1862, 315.

Tropidinius (Gill) Poey, 1868, 353.

Neomesoprion Castelnau, 1875, 378.

Rabirubia Jordan \& Fesler, 1893, 462.

Raizero Jordan \& Fesler, 1893, 462.

Bowersia ${ }^{867}$ Jordan \& Evermann, 1903,

504.

Bennettia Fowler, 1904, 507.
Parkia Fowler, 1904, 507.

Etelides Jordan \& J. C. Thompson, 1905, 514.

Rooseveltia Jordan \& Evermann, 1906, 519.

Ulaula ${ }^{388}$ Jordan \& W. F. Thompson, 1911

Etelinus Jordan \& W. F. Thompson, 1911, 539.

Vegetichthys Tanaka, 1918, 565.

Rhomboplitoides ${ }^{809}$ Fowler, 1918.

${ }^{867}$ Platyinus and Bozersia are apparently synonymous with Pristipomoides.

${ }^{308}$ Jordan \& W. F. Thompson, Proc. U. S. Nat. Mus., 39: 459, 1911; type Bowersia ulaula Jordan \& Evermann, Chatopterus sieboldi Bleeker; replaces Chatopterus Temminck \& Schlegel, preoccupied.

${ }^{3}$ Fowler, Proc. Ac. Nat. Sci. Phila., $70: 33$; orthotype R. megalops Fowler. (Omitted in "Genera of Fishes.")

\section{Family 442. VERILID正}

Verilus Poey, 1860, 298.

\section{Family 443. APHAREID王}

Aphareus Cuvier \& Valenciennes, 1830,

135.

\section{Family 444. POMADASIDE (Hamulida; Pristipomida)}

(Grunts)

Ghanan Forskål, 1775, 34.

Gaterin Forskå1, 1775, 34.

Cæsio Lacépède, 1802, 61.

Plectorhinchus ${ }^{370}$ Lacépède, 1802, 61.

Pomadasys Lacépède, 1803, 64.

Cheloniger (Plumier) Lacépède, 1803, 73.

Diagramma Cuvier, 1815, 94.

Scolopsis ${ }^{371}$ Cuvier, 1815, 94.

Pristipomus $^{372}$ (Cuvier) Oken, 1817, 103.

Diabasis Desmarest, 1823, 116.

Anomalodon Bowdich, 1825, 119.

Hæmulon ${ }^{878}$ Cuvier, 1829, 128.

Conodon Cuvier \& Valenciennes, 1830, 135.

Lycogenis (Kuhl \& Van Hasselt) Cuv. \& Val., 1830, 174.

Hapalogenys Richardson, 1844, 222.

Pinjalo Bleeker, 1845, 225.

Heterodon Bleeker, 1845, 225.

Heterognathodon Bleeker, 1848, 233.
Hyperoglyphe Günther, 1859, 291.

Odontonectes Günther, 1859, 291.

Orthopristis Girard, 1859, 291.

Apostata (Heckel) Canestrini, 1860, 294.

Anisotremus Gill, 1860, 301.

Orthostœchus Gill, 1862, 316.

Microlepidotus Gill, 1862, 316.

Genytremus Gill, 1862, 316.

Genyatremus Gill, 1862, 316.

Pristocantharus Gill, 1862, 316.

Brachydeuterus Gill, 1862, 313.

Anarmostus Scudder, 1863, 328.

Bathystoma Scudder, 1863, 328.

Brachygenys (Scudder) Poey, 1868, 353.

Hæmulopsis Steindachner, 1869, 355.

Parapristipoma Bleeker, 1873, 367.

Paracæsio Bleeker, 1875, 378.

Paraconodon Bleeker, 1876,382.

Gymnocæsio Bleeker, 1876, 382.

Liocæsio Bleeker, 1876, 382.

${ }^{\mathrm{a} 70}$ Written Plectorhynchus by authors.

${ }^{27}$ Later called Scolopsides.

${ }^{372}$ Later written by Cuvier Pristipoma.

${ }^{37}$ Also written incorrectly $\mathrm{Hamylum}$. 
Pterocæsio Bleeker, 1876, 382.

Pseudopristipoma Sauvage, 1880, 405.

Propoma Günther, 1880, 403.

Lythrulon Jordan \& Swain, 1884, 428.

Eurumetopos Morton, 1887, 438.

Isaciella Jordan \& Fesler, 1893, 462.

Isacia Jordan \& Fesler, 1893, 462.

Rhonciscus Jordan \& Evermann, 1896, 472.

Rhencus J. \& E., 1896, 472.
Evapristis J. \& E., 1896, 472.

Parascolopsis Boulenger, 1901, 492.

Mylacrodon Regan, 1903, 505.

Euelatichthys Fowler, 1904, 508.

Spilotichthys Fowler, 1904, 508.

Diagrammella Pellegrin, 1912, 544, 549.

Leptoscolopsis Tanaka, 1915, 558.

Otoperca Boulenger, 1916, 558.

Dacymba Jordan \& Hubbs, 1917, 562.

\section{Fanily 445 . THERAPONID $\not E$}

Djabub Forskål, 1775, 34.

Therapon ${ }^{374}$ Cuvier, 1817, 104.

Coius Hamilton, 1822, 172.

Helotes Cuvier, 1829, 127.

Pelates Cuvier, 1829, 126.

Pterapon Gray, 1833, 139, 179.

Mesopristes ${ }^{378}$ Bleeker, 1845, 225, 382.
Datnioides (Brisout de Barneville)

Canestrini, 1860, 294.

Pseudohelotes Guimaraës, 1882, 419.

Autisthes De Vis, 1885, 431.

Hephæstus De Vis, 1885, 431.

Plagiogeneion Forbes, 1890, 450.

Eutherapon Fowler, 1904, 508.

${ }^{374}$ Misprinted Terapon.

${ }^{875}$ Replaces Datnia Cuvier \& Valenciennes, which is not the same as Datnia Cuvier, the latter a synonym of Sparus Linnæus.

\section{Family 446. BANJOSID $2 \mathrm{E}$}

Anoplus Temminck \& Schlegel, 1842, 213. Banjos Bleeker, 1876, 382.

\section{Family 447. LETHRINID丑}

Schour Forskål, 1775, 34

Lethrinus Cuvier, 1829, 128.

Pentapus ${ }^{278}$ Cuvier \& Valenciennes, 1830. 258.

Monotaxis Bennett, 1830, 136.

Leiopsis Bennett, 1830, 174.
Sphœrodon Rüppell, 1830, 184.

Mænioides ${ }^{\text {s7t }}$ Richardson, 1843.

Maina Gistel, 1848, 236.

Lethrinella Fowler, 1904, 508.

Lethrinichthys Jordan \& Thompson, 1912 , 544.

${ }^{376}$ Pentapus is transferred (with its synonyms, Leiopsis and Manoides) from the Pomadasida to this group by Regan.

${ }^{877}$ Richardson, "Icones Piscium," 8; logotype M. aurofrenatus Richardson = Sparus vittatus Cuvier \& Valenciennes, a synonym of Pentapus. (Omitted in "Genera of Fishes.") (McCulloch.)

\section{Family 448. NEOLETHRINID\&E}

Palate and whole roof of mouth said to be covered with small molar teeth.

Neolethrinus Castelnau, 1875, 378 .

\section{Family 449. SPARID $\approx$}

(Pargos)

The genera related to Sparus are very differently distributed by Regan from a study of the skeletons. The present arrangement, mainly according 
dentition, approximates more nearly the earlie rarrangement of Günther and is at least not unnatural. Regan adds to this group all the Mediterranean genera (Boops, Scatharus, Oblada, etc.) here placed in Girellida. He also adds Dentex and its relatives, leaving Nemipterus (and its synonyms) with Scolopsis and Heterognathodon to form a separate family of $\mathrm{Ne}$ mipteridce.

Sparus Linnæus, 1758, 13.

Cynadus Gronow, 1763, 19.

Aurata Catesby, 1771, 30.

Sargus Klein, 1775, 39.

Cynædus (Gronow) Scopoli, 1777, 42.

Mylio (Commerson) Lacépède, 1802, 70.

Diplodus Rafinesque, 1810, 82.

Sargus Cuvier, 1817, 102.

Pagrus Cuvier, 1817, 103.

Aurata (Cuvier) Oken, 1817, 103.

Labeo Bowdich, 1825, 118.

Aurata Risso, 1826, 120.

Charax Risso, 1826, 173.

Daurada Stark, 1828, 123.

Pagellus Cuvier, 1828, 128.

Datnia ${ }^{378}$ Cuvier, 1829, 126.

Chrysophris ${ }^{378}$ Cuvier, 1829, 128.

Boridia ${ }^{380}$ Cuvier \& Valenciennes, 1830 , 135.

Sparnodus Agassiz, 1838, 191.

Chrysoblephus Swainson, 1839, 199.

Spri.rodus Agassiz, 1839, 194.

Argyrops Swainson, 1839, 199.

Lithognathus Swainson, 1839, 199.

Calamus Swainson, 1839, 199.

Sclanurus Agassiz, 1845, 223.

Radamas Münster, 1846, 229.

Asima Giebel, 1848, 233, 242.
Cæso Gistel, 1848, 235.

Denius Gistel, 1848, 237.

Eudynama Gistel, 1848, 238.

Trigonodon Sismonda, 1849, 244.

Lagodon Holbrook, 1855, 265.

Dich elodus Giebel, 1857, 409.

Pagrichthys Bleeker, 1860, 293.

Lobodus Costa, 1866, 340.

Symphorus Günther, 1872, 365 .

Grammateus Poey, 1874, 376.

Sparoides Probst, 1874, 376.

Puntazzo Bleeker, 1876, 383.

Sparosoma Sauvage, $1883,425$.

Pseudosph arodon Noetling, 1885, 434.

Pagrosomus Gill, 1893, 461.

Sparosomus Gill, 1893, 461.

Otrynter Jordan \& Evermann, 1896, 472.

Salema J. \& E., 1896, 472.

Calopomus (Agassiz) Woodward, (1845) 1901, 224.

Taius Jordan \& Thompson, 1912, 544.

Evynnis ${ }^{381}$ J. \& T., 1912, 544.

Parargyrops $^{382}$ Tanaka, 1916, 560.

Stenesthes Jordan, 1917, 562.

Rhyth Mias Jordan \& Gilbert, 1920, 571.

Plectrites Jordan, 1920, 571.

Atkinsonella Jordan, 1920, 572.

${ }^{378}$ The original type species of this genus is said to be a Sparus.

${ }^{379}$ Also written Chrysophrys.

${ }^{380}$ This little genus is placed by Regan with the Pomadasida.

${ }^{381}$ This genus, otherwise close to Pagrosomus, differs from all other Sparoid fishes in having a few stout conical teeth on the vomer.

${ }^{362}$ Misprinted Paragyrops in "Genera of Fishes."

\section{Family 450. DENTICID压 (Nemipterida)}

Regan refers Dentex to the Sparida, leaving most of the other genera to form the Nemipterida.

Synagris Klein, 1775, 38.

Dentex Cuvier, 1815, 94.

Nemipterus Swainson, 1839, 199.

Synagris Günther, 1859, 291.

Sparopsis $^{383}$ Kner, 1868, 352.

Gymnocranius Klunzinger, $1870,358$.

${ }^{8 \otimes}$ Sparopsis and Anemura are apparently synonymous with Synagris as restricted. 
Polysteganus Klunzinger, 1870, 358.

Gnathodentex Bleeker, 1873, 367.

Paradentex Bleeker, 1876, 382.

Synagris (Klein) Bleeker, 1876, 383.
Ctenodentex Storms, 1896, 475, 484.

Anemura ${ }^{393}$ Fowler, 1904, 507.

Odontoglyphis Fowler, 1904, 507.

Euthyopteroma Fowler, 1904, 507.

\section{Family 451. GIRELLID $\mathbb{E}$}

Vegetable-feeders with incisor teeth, mostly movable, and no molars.

Coracinus Gronow, 1763, 19.

Boops Cuvier, 1815, 94.

Cantharus Cuvier, 1817, 103.

Dipterodon Cuvier, 1829, 128.

Oblada Cuvier, 1829, 128.

Crenidens Cuvier \& Valenciennes, 1830, 135.

Scatharus Cuv. \& Val., 1830, 135.

Box Cuv. \& Val., 1830, 135.

Sarpa Bonaparte, 1831, 175.

Girella Gray, 1833, 179.

Exocallus De la Pylaie, 1835, 183.

Melanichthys Temminck \& Schlegel, 1844, 223.

Spondyliosoma Cantor, 1849, 241.
Camarina Ayres, 1854, 255.

Doydixodon Valenciennes, 1855, 266.

Gymnocrotaphus Günther, 1859, 291.

Proteracanthus Günther, 1859, 291.

Tephræops Günther, 1859, 291.

Pachymetopon Günther, 1859, 291.

Incisidens Gill, 1862, 316.

Melambaphes Günther, 1863, 326.

Glyphodes Guichenot, 1864, 332.

Tripterodon Play fair, 1866, 343.

Girellich thys Klunzinger, 1872, 366.

Neotephræops Castel nau, 1872, 362.

Aplodon Duméril, 1883, 426.

Dichistius Gill, 1888, 441.

Girellops Regan, 1913, 550.

\section{Family 452. KYPHOSID压}

\section{(Pilot Fishes)}

Tahhmel Forskål, 1775, 34.

Kyphosus Lacépède, 1802, 61.

Pimelepterus Lacépède, 1803, 63.

Xyster Lacépède, 1803, 68.

Dorsuarius Lacépède, 1803, 68.
Xysterus Rafinesque, 1815, 90.

Seleima Bowdich, 1825, 119.

Opisthistius Gill, 1862, 316.

Sectator Jordan \& Fesler, 1893, 462.

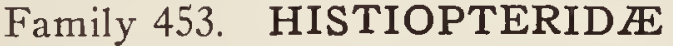

(Boar Fishes)

Pentaceros Cuvier, 1829, 125.

Histiopterus $^{384}$ Temminck \& Schlegel, 1843.

Richardsonia Castelnau, 1872, 362.

Pseudopentaceros Bleeker, 1876, 382.

Paristiopterus Bleeker, 1876, 382.

Prosoplismus Waite, 1903, 506.

Zanclistius Jordan, 1907, 524.

Evistias Jordan, 1907, 524.

Quadrarius Jordan, 1907, 524.

Quinquarius Jordan, 1907, 524.

Gilchristia Jordan, 1907, 524.

Pentaceropsis Steindachner \& Döderlein, $1883,426$.

${ }^{384}$ "Fauna Japonica," 86; orthotype Histiopterus typus Temminck \& Scllegel. (Omitted in "Genera of Fishes.")

Enoplosus Lacépède, 1803, 64.

\section{Family 454 . ENOPLOSID蚱}

\section{Family 455. INERMIID $\approx$}

This small family has no visible relation to the Emmelichthyida, to which group the genus Inermia has been referred. The narrow maxillary is 
devoid of scales and slips under the preorbital. The dorsal fins are separate, the first of many spines.

Dipterygonotus Bleeker, 1849, $240 . \quad$ Inermia Poey, 1861, 299.

\section{Family 456. MANIDE (Merolepida)}

Manas Klein, 1775, 39.

Spicara Rafinesque, 1810, 80.

Centracanthus ${ }^{\mathrm{885}}$ Rafinesque, 1810, 80.

Merolepis Rafinesque, 1810, 81.
Smaris Cuvier, 1815, 93.

Mæna Cuvier, 1829, 128.

Mænas (Klein) Bleeker, 1876, 382.

${ }^{885}$ Later spelled Centracantha, originally misspelled Centracantus.

\section{Family 457. EMMELICHTHYIDE (Erythrichthyida)}

This family differs widely from the Manida in the large, scaly uncovered maxillary. The dorsal fin is divided into two.

Emmelichthys Richardson, 1844, 223.

Erythrichthys Temminck \& Schlegel, $1845,227$.
Boxaodon Guichenot, 1848, 238.

Plagiogeneion Forbes, 1890, 450 .

Erythrocles Jordan, 1919, 567.

\section{Family 458. GERRID $Æ$ (Xystamida)}

\section{(Mojarras; Silver Perch)}

Mormyrus Catesby, 1771, 29.

Gerres (Cuvier) Quoy \& Gaimard, 1824, 117.

Diapterus Ranzani, 1840, 208.

Podager Gistel, 1848, 236.

Catochænum Cantor, 1849, 241.

Pentaprion Bleeker, 1850, 245.

Eucinostomus Baird, 1857, 274.

Synistius Gill, 1862, 315.
Clara Gill, 1862, 314.

Moharra Poey, 1875, 379.

Gerreomorpha Alleyne \& Macleay, 1877, 387.

Parequula Steindachner, 1879, 401.

Chthalmopteryx Ogilby, 1887, 438.

Xystæma Jordan \& Evermann, 1895, 468.

Ulæma J. \& E., 1895, 468.

Pertica Fowler, 1904, 508.

\section{Family 459. OPLEGNATHID $\approx$}

Oplegnathus Richardson, 1840, 208.

Scarostoma Kner, 1867, 346.

Scaradon Temminck \& Schlegel, 1844, 223.

\section{Family 460. MULLIDE} (Surmullets)

Mullus Linnæus, 1758, 14.

Upeneus ${ }^{888}$ Cuvier, 1829, 127.

Mulloides Bleeker, 1849, 240.

Upeneoides Bleeker, 1849, 240.

Megalepis Bianconi, 1855, 262.

Upeneichthys Bleeker, 1855, 263.
Acanthomullus Valenciennes, 1861, 309.

Pseudupeneus Bleeker, 1862, 310.

Parupeneus Bleeker, 1863, 322.

Mullhypeneus Poey, 1867, 347.

Brachymullus Bleeker, 1876, 383.

Pseudomulloides Ribeiro, 1915, 557, 560.

${ }^{286}$ Name corrected to Hypeneus by apthors. 


\section{Family 461. SCIENIDAE}

(Croakers; Roncadores)

Vertebræ of the usual number, $10+14$.

Sciæna Linnæus, 1758, 13

Alburmus Catesby, 1771, 30.

Cromis Browne, 1789, 47.

Johnius Bloch, 1793, 51 .

Lonchiurus seas Bloch, 1793, 51.

Pogonias Lacépède, 1802, 61.

Leiostomus seb Lacépède, 1803, 64.

Pogonathus Lacépède, 1803, 65.

Chromis (Plumier) Lacépède, 1803, 73.

Coracinus Pallas, 1811, 84.

Equietus Rafinesque, 1815, 89.

Stellifer (Cuvier) Oken, 1817, 104.

Umbrina Cuvier, 1817, 104.

Aplodinotus $^{\text {sscc }}$ Rafinesque, 1819, 110.

Amblodon Rafinesque, 1819, 110.

Bola Hamilton, 1822, 114.

Stelliferus Stark, 1828, 123.

Pachyurus Agassiz, 1829, 132.

Corvina Cuvier, 1829, 128.

Larimus Cuvier \& Valenciennes, 1830, 134.

Micropogon Cuv. \& Val.,1830, 135.

Lepipterus Cuv. \& Val., 1830, 135.

Micropogonias Bonaparte, 1831, 175.

Argyrosomus De la Pylaie, 1835, 184.

Cheilotrema Tschudi, 1846, 230.

Apeches Gistel, 1848, 236.

Melantha Gistel, 1848, 238.

Attilus Gistel, 1848, 238.

Homoprion Holbrook, 1855, 265.

Sciænoides Blyth, 1860, 294.

Collichthys Günther, 1860, 296.

Rhinoscion Gill, 1861, 304.

Plagioscion Gill, 1861, 304.

Anomiolepis Gill, 1861, 304.

Menticirrhus Gill, 1861, 304.

Pachypops Gill, 1861, 304.

Genyonemus Gill, 1861, 304.

${ }^{8802}$ Often written Lonchurus.

${ }^{3800}$ Often written Liostomus.

${ }^{28 s e}$ Corrected to Haploidonotus by Gill.
Haploidonotus (Rafinesque) Gill, 1861, 304.

Bairdiella Gill, 1861, 302.

Pseudotolithus Bleeker, 1861, 321.

Odontoscion Gill, 1862, 313.

Cirrhimens Gill, 1862, 313.

Amblyscion Gill, 1863, 324.

Ophioscion Gill, 1863, 324.

Diplolepis Steindachner, 1863, 328.

Hemisciæna Bleeker, 1863, 321.

Pseudosciæna Bleeker, 1863, 321.

Scianops Gill, 1863, 323.

Polycirrhus Bocourt, 1869, 354.

Paralonchurus Bocourt, 1869, 354.

Larimodon (Kaup) Bleeker, 1876, 383.

Eutychelithus Jordan, 1876, 386.

Pareques Gill, 1876, 385.

Roncador Jordan \& Gilbert, 1880, 403.

Corvula Jordan \& Eigenmann, 1889, 447.

Callaus Jordan \& Eigenmann, 1889, 447.

Polyclemus Berg, 1895, 466.

Asperina Ostroumoff, 1896, 475.

Elattarchus Jordan \& Evermann, 1896, 472.

Zonoscion J. \& E., 1896, 472.

Zaclemus Gilbert, 1896, 473.

Nector Jordan \& Evermann, 1898, 480.

Zestis J. \& E., 1898, 481.

Zestidium J. \& E., 1898, 481.

Stellicarens J. \& E., 1898, 481.

Sigmurus Gilbert, 1898, 481.

Cilus Delfin, 1900, 489.

Larimichthys Jordan \& Starks, 1905, 514.

Pseudomycterus Ogilby, 1908, 529.

Twingonia Pascoe, 1908, 529.

Othonias Jordan \& Thompson, 1911, 539.

Nibea J.\& T., 1911, 539. 


\section{Family 462. OTOLITHID王}

(Weak Fishes; Queen Fishes)

Vertebræ $14+10$, not $10+14$ as in Scianida and the types allied to Scicena, Serranus, Sparus, and Lutianus.

Otolithes $^{87}$ (Cuvier) Oken, 1817, 104.

Ancylodon (Cuvier) Oken, 1817, 104.

Macrodon Schinz, 1822, 115.

Nebris Cuvier \& Valenciennes, 1830, 134.

Nomalus Gistel, 1848, 234.

Seriphus Ayres, 1854, 255.

Cestreus Gronow, 1854, 258.

Cynoscion Gill, 1861, 304.

Atractoscion Gill, 1862, 313.

Archoscion Gill, 1862, 313.

Apseudobranchus Gill, 1862, 313.
Isopisthus Gill, 1862, 313.

Paraplesichthys (Kaup) Bleeker, 1876, 383.

Sagenichthys Berg, 1895, 466.

Buccone Jordan \& Evermann, 1896, 472.

ARCh жotolith us Stolley, 1910, 537.

Symphysoglyphus Ribeiro, 1913, 551.

Lom POQUiA Jordan \& Gilbert, 1919, 569.

Aristoscion Jordan, 1920, 571.

Ioscion $^{388}$ Jordan, 1921.

${ }^{857}$ Later written Otolithus by Cuvier. Jordan.

${ }^{388}$ Jordan, "Fishes of the California Tertiary," 283; orthotype Ioscion morgani

\section{Family 463. SILLAGINID无}

Sillago Cuvier, 1817, 101.

Sillaginodes Gill, 1861, 306.

Sillaginopsis Gill, $1861,306$.

Neosillago Castelnau, 1875, 378.

Iosillago Macleay, 1878, 396.

\section{Family 464. NANDID $巴$}

This group may require further subdivision.

Nandus Cuvier \& Valenciennes, 1831, 137.

Bedula Gray, 1833, 179.

Pomanotis Guichenot, 1847, 232.

Acharnes Müller \& Troschel, 1848, 239.
Pristolepis Jerdon, 1848, 239, 246.

Catopra Bleeker, 1851, 248.

Badis Bleeker, 1853, 253.

Paranandus Day, 1865, 336.

\section{Family 465. POLYCENTRID尼}

Monocirrhus Heckel, 1840, 207.

Polycentrus Müller \& Troschel, 1848, 239.

Polycentropsis Boulenger, 1901, 492.

\section{Family 466. MALACANTHID E}

This family and the next four bear strong resemblance to the trachiniform fishes, differing mainly in the thoracic insertion of the ventrals.

Malacanthus Cuvier, 1829, 130

Oceanops Jordan \& Seale, 1906, 519.

\section{Family 467. BRANCHIOSTEGIDE (Latilida)}

Coryphænoides Lacépède, 1802, 62.

Branchiostegus Rafinesque, 1815, 90.

Latilus Cuvier \& Valenciennes, 1830, 135.

Caulolatilus Gill, 1862, 316.
Dekaya Cooper, 1863, 323.

Prolatilus Gill, $1865,337$.

Lopholatilus Goode \& Beane, 1879, 400.

Hoplolatilus Günther, 1887, 438. 


\section{Family 468. LABRACOGLOSSID}

Platystethus Günther, 1860, 296.

Labracoglossa ${ }^{389}$ Peters, 1866, 342.

Cypselichthys Steindachner \& Döderlein, $1883,426$.
Evistius Gill, 1893, 461.

Bathystethus Gill, 1893, 461.

${ }^{889}$ This genus is without teeth in the gullet and has no apparent affinity with the Stromateida.

\section{Family 469. CEPOLID五}

Cepola Linnæus, 1764, 22.

Acanthocepola Bleeker, 1874, 372.

Tænia Röse, 1793, 52.

\section{Family 470. HENICICHTHYID瓜}

Henicichthys ${ }^{300}$ Tanaka, 1915, 558.

${ }^{300}$ Description in Japanese. The following is the translation as furnished me by Mr. Tanaka: Henichthys foraminosus (Henichthyidce new family) proposed in Japanese in Zool. Mag., 27 : No. 325, Nov. 15, 1915, p. 568. Head 24-5 in length (exclusive of caudal), depth 53-8. Eye lateral; mouth large, somewhat oblique; maxillary extending behind posterior border of eye; teeth in jaws acute, arranged in a row; vomer and palatines toothed. Two dorsals somewhat close together. Dorsal, VI, 11; anal, 11; pectoral 13; ventral I, 5 . Ventral fins close together, beneath pectoral; caudal nearly truncated. Scaleless; body and head with many pores which are arranged longitudinally and transversely. Color pale, plain. Length (exclusive of caudal) $5-5 \mathrm{~cm}$. Nagasaki. The species seems to be a trachinoid fish and also to be included in a new family.

\section{Series TRICHODONTIFORMES \\ Family 471. TRICHODONTIDE}

The trachinoid fishes (Jugulares) follow in a natural sequence, a fact not to be shown in a linear series.

Trichodon (Steller) Cuvier, 1829, 127.

Chætichthys Gistel, 1848, 238.

Arctoscopus Jordan \& Evermann, 1896, 474.

\section{Suborder CIRRHITOIDEI \\ Family 472. CIRRHITID王}

The fishes of this group bear some resemblance to the Cataphracti, which may be an indication of common origin.

Cirrhitus ${ }^{200 a}$ Lacépède, 1803, 65.

Cirrhitichthys ${ }^{3000}$ Bleeker, 1856, 274.

Oxycirrhites Bleeker, 1857, 274.

Amblycirrhitus Gill, 1862, 313.

Cirrhitopsis Gill, 1862, 313.

Lacepedia Castelnau, 1873, 367.

Neocirrhites Castelnau, 1873, 368.

Paracirrhites Bleeker, 1875, 378.
Zeodrius Castelnau, 1878, 393.

Paracirrhites Steindachner \& Döderlein, $1883,426$.

Psilocranium Macleay, 1884, 429.

Dactylophora De Vis, 1884, 427.

Cirrhitoidea Jenkins, 1903, 504.

Isobuna Jordan, 1907, 525.

Cyprinocirrhites Tanaka, 1917, 563.

${ }^{8008}$ Written Cirrhites by authors.

${ }^{8000}$ This name appears first in Ned. Tydschr. Dierk., 10:474, 1856. 


\section{Family 473. CHIRONEMIDE}

Chironemus Cuvier, 1828, 126.

Threpterius Richardson, 1850, 247.
Scicnoides (Solander) Richardson, 1850 , 247.

\section{Family 474. CHEILODACTYLID王}

Cheilodactylus Lacépède, 1803, 65.

Nemadactylus ${ }^{321}$ Richardson, 1839, 197.

Pteronemus Van der Hoeven, 1849, 243, 383.

Trichopterus Gronow, 1854, 259.
Chirodactylus Gill, 1862, 313.

Acantholatris Gill, 1862, 313.

Dactylopagrus Gill, 1862, 313.

Dactylosparus Gill, 1862, 314.

${ }^{801}$ Spelled also Nematodactylus.

\section{Family 475. APLODACTYLIDÆ}

Aplodactylus ${ }^{822}$ Cuvier \& Valenciennes, 1831, 137.

Crinodus Gill, 1862, 313.

Dactylosargus Gill, 1862, 313.

Goniistius Gill, 1862, 314.

Parhaplodactylus Thominot, 1883, 426.

${ }^{202}$ Often written Haplodactylus.

\section{Family 476. LATRIDÆE}

Latris Richardson, 1839, 197.

Mendosoma Guichenot, 1848, 238.

Latridopsis Gill, 1862, 314.

\section{Series GADOPSIFORMES}

Family 477. GADOPSID E

Gadopsis Richardson, 1844, 223.

\section{Series CAPRIFORMES}

A small group of uncertain relations, bearing a strong resemblance to the Zeide in form and having, like the Zeida and the Chotodontide, the post-temporal solidly joined to the skull. But the ventral rays are always I, 5, as in the Chetodonts, and the gill membranes free from the isthmus, as in Zeus. A comparative study of the skeletons of these transitional groups, Zeus, Capros, Toxotes, Scorpis, Ephippus, Platax, Drepane, and Amphistium, is much to be desired.

\section{Family 478. CAPROID $\$$}

Capros Lacépède, 1803, 65.

Caprophonus Müller \& Troschel, 1849, 243.

\section{Family 479.}

Antigonia Lowe, 1843, 215.

Hypsinotus Temminck \& Schlegel, 1844, 223.

\section{Family 480. AMPHISTIID}

A group of uncertain relations, apparently allied to the Ephippida, regarded by Boulenger as an ally of Zeus and perhaps ancestral to the 
Heterosomata. As Regan finds the ventrals I, 5, we have separated it from the zeoid fishes.

A mphistium Agassiz, 1835, 181, $218 . \quad$ Macrostoma ${ }^{803}$ Agassiz, 1838, 191.

${ }^{393}$ A genus of doubtful affinity, resembling a chætodont, the name preoccupied.

\section{Series EPHIPPIFORMES}

This group has many characters in common with the perciform fishes, especially in having the post-temporal not solidly united to the skull. It agrees, however; with the Squamipennes in many regards, notably the restriction of the gill openings to the sides; the compressed body and scaly fins mark a transition from the Scorpida and related forms to the Che todontida.

\section{Family 481. SCORPID正}

(Half-moon Fishes)

Scorpis Cuvier \& Valenciennes, 1831, 573. Agenor Castelnau, 1878, 393.

Atypus Günther, 1860, 296.

Atypichthys Günther, 1862, 575.

Diodyxodon Thominot, 1881, 417.

Helotosoma Kaup, 1863, 327.

Parapsettus Steindachner, 1875, 381.

Parascorpis Bleeker, 1875, 377.

Cæsiosoma Kaup, 1876, 383.

Tilodon Thominot, 1881, 417.

Neptotichthys Hutton, 1890, 451.

Medialuna Jordan \& Fesler, 1893, 462.

Atyposoma Boulenger, 1899, 485.

Neatypus Waite, 1905, 516.

\section{Family 482. MONODACTYLID $\mathbb{E}$}

A group of uncertain affinity, seeming close to the Xyphoside on the one hand and to the chætodontoid forms on the other. I place them provisionally with the Ephippida, but it may be that the genera will require to be rearranged. From the allies of Chatodon, the Scorpida, Monodactylide, Platacida, Drepanida, and Ephippida differ in not having the posttemporal coössified with the skull.

Monodactylus Lacépède, 1802, 61.

Centropodus Lacépède, 1802, 62.

Psettus (Commerson) Lacépède, 1802, 70.

Acanthopodus Lacépède, 1803, 64.

Platax Cuvier, 1817, 105.

\section{Family 483. PLATACID尼}

\section{Family 484. EPHIPPID E}

Chætodipterus Lacépède, 1803, 64.

Ephippus Cuvier, 1817, 105.

Ilarches Cantor, 1849, 241.

Parephippus Gill, 1861, 305.

Ephippites Von Ammon, 1901, 492.

Bramichthys Waite, 1905, 516.
Schuettea Steindachner, 1866, 343.

Psettias Jordan, 1906, 519.

\section{Family 485. DREPANID $\mathbb{E}$}

Drepane Cuvier \& Valenciennes, 1831,136 ,

175.

Enixe Gistel, 1848, 235.

Harpochirus Cantor, 1849, 241.

Drepanichthys ${ }^{394}$ Bonaparte, 1831.

Cryptosmilia Cope, 1867, 344.

${ }^{304}$ Bonaparte, "Saggio," etc. A substitute for Drepane, as are also Enixe and Harpochirus, Drepane being regarded as preoccupied by the earlier names, Drepana, Drepania, Drepanus, Drepanis, and Drepanius. Drepanichthys is quoted by Agassiz, "Nomenclator," 23, 1843. (Overlooked in "Genera of Fishes.") 


\section{Suborder SQUAMIPENNES \\ (Epelasmia)}

Post-temporal firmly attached to the skull, the interspace between the three forks filled in by bone; gill openings restricted to the sides; soft fins largely scaly.

\section{Series TOXOTIFORMES}

\section{Family 486. TOXOTID瓜}

This family, of uncertain relationship, agrees with the Chcetodontidce, according to Günther in the union of the post-temporal with the skull.

Toxotes Cuvier, 1817, 105.

Amblytoxotes Bleeker, 1876, 383.

Trompe Gistel, 1848, 237.

\section{Seriès CHAETODONTIFORMES}

\section{Family 487. SCATOPHAGID $\approx$}

In this family the post-temporal is solidly united to the skull as in Chetodontida.

Scatophagus Cuvier \& Valenciennes, 1831, Prenes Gistel, 1848, 237. 136.

Cacodoxus Cantor, 1849, 241.

\section{Family 488. CHETODONTID王}

(Butterfly Fishes; Coral Fishes)

Chætodon Linnæus, 1758, 13.

Acarauna Catesby, 1771, 32.

Tetragonoptrus Klein, 1775, 40.

Holacanthus Lacépède, 1803, 64.

Pomacanthus Lacépède, 1803, 64.

Chelmon ${ }^{385}$ Cuvier, 1817, 105.

Heniochus Cuvier, 1817, 105.

Taurichthys Cuvier, 1829, 128.

Rabdophorus Swainson, 1839, 198.

Microcanthus ${ }^{396}$ Swainson, 1839, 198.

Genicanthus Swainson, 1839, 198.

Megaprotodon Guichenot, 1848, 238.

Diphreutes Cantor, 1849, 241.

Centropyge Kaup, 1860, 298.

Linophora Kaup, 1860, 297.

Eteira Kaup, 1860, 297.

Citharœdus Kaup, 1860, 297.

Coradion Kaup, 1860, 297.

Therapaina Kaup, 1860, 298.

Sarothrodus Gill, 1861, 304, 316.

Prognathodes Gill, 1862, 316.
Pomacanthodes Gill, 1862, 316.

Tholichthys Günther, 1868, 351.

Tetragonoptrus (Klein) Bleeker, 1868, $348,383$.

Neochætodon Castelnau, 1873, 368.

Parachætodon Bleeker, 1874, 372.

Oxychætodon Bleeker, 1876, 383.

Chætodontops Bleeker, 1876, 383.

Lepidochætodon Bleeker, 1876, 383.

Acanthochætodon Bleeker, 1876, 383.

Chætodontoplus Bleeker, 1876, 383.

Gonochætod on Bleeker, 1876, 383.

Hemitaurichthys Bleeker, 1876, 383.

Chelmonops Bleeker, 1876, 383.

Hemichætodon Bleeker, 1876, 384.

Anisochætod on Klunzinger, 1884, 429.

Angelichthys Jordan \& Evermann, 1896, 473.

Forcipiger Jordan \& McGregor, 1898, $481,482$.

Osteochromis Franz, 1910, 535.

${ }^{806}$ Later written Chelmo.

${ }^{308}$ A misprint for Micracanthus. 
Vinculum McCulloch, 1914, 554.

Loa ${ }^{307}$ Jordan, 1921.
Tifia ${ }^{398}$ Jordan, 1923.

Xiphypops ${ }^{309}$ Jordan, 1923.

${ }^{307}$ Jordan, Proc. U. S. Nat. Mus., 653, 1921; type Loa excelsa Jordan.

${ }_{898}$ "Fishes of Hawaii," Mem. Carnegie Museum, 1923, 60; orthotype Chatodon corallicola Snyder.

${ }^{320}$ Loc. cit., 64; orthotype Holacanthus fisheri Snyder.

\section{Series ZANCLIFORMES}

\section{Family 489. ZANCLIDIE}

Zanclus (Commerson) Lacépède, 1803, 70.

$Z$ anclus (Commerson) Cuvier \& Valenciennes, 1831, 136.

\section{Series ACANTHURIFORMES}

Family 490. ACANTHURID正 (Teuthidida; Hepatide)

Hepatus $^{400}$ Gronow, 1763, 20.

Teuthis ${ }^{401}$ Linnæus, 1766; 23.

Rhombotides Klein, 1775, 38.

Acanthurus Forskål, 1775, 33.

Harpurus Forster, 1778, 43.

Teuthis Browne, 1789, 48.

Monoceros Bloch \& Schneider, 1801, 58.

Naso Lacépède, 1802, 61.

Naseus (Commerson) Lacépède, 1802, 69.

Aspisurus Lacépède, 1803, 64.

Prionurus Lacépède, 1804, 169.

Nasonus Rafinesque, 1815, 88.

Chirurgus Blainville, 1818, 108.

Priodon Qiny \& Gaimard, 1824, 118.

Axinurus Cuvier, 1829, 129.

Priodontichthys Bonaparte, 1831, 175.

Scopas Bonaparte, 1831, 175.

Ctenodon Bonaparte, 1833, 175.
Gonopterus Gronow, 1854, 258.

Gnathocentrum Guichenot, 1866, 341.
Keris ${ }^{402}$ Cuvier \& Valenciennes, 1835, 181.

Zebrasoma Swainson, 1839, 201.

Callicanthus Swainson, 1839, 201.

Ctenodon Swainson, 1839, 201.

Acronurus Gronow, 1854, 259.

Acronurus (Gronow) Günther, 1861, 307.

Rhombotides (Klein) Bleeker, 1863, 322.

Scopas $^{403}$ (Bonaparte) Kner, 1865.

Calamostoma Steindachner, 1863, 328.

Ctenodon (Bonaparte) Klunzinger, 1884, 429.

Ctenochætus Gill, 1884, 428.

Colocopus Gill, 1884, 428.

Apostasis Kramberger, 1891, 455.

Xesurus Jordan \& Evermann, 1896, 473.

Acanthocaulus Waite, 1900, 491.

Hepatus (Gronow) Jordan \& Seale, 1906.

Læphichthys Ogilby, 1916, 560.

${ }^{400}$ If Gronow's names (1763) are accepted Hepatus would replace Acanthurus. The original Teuthis of Linnæus, 1768, was exactly equivalent to the non-binomial Hepatus of Gronow. The generic distinction between the two species, hepatus and javus, was recognized by Forskål, 1775, who named the type of the former Acanthurus, the other Siganus. Cuvier \& Valenciennes used the names Acanthurus and Amphacanthus. Neither of these writers adopted Teuthis as a generic name. The first restriction of Teuthis was that of Cantor, who used it for the Siganus group, although by good rights he should have taken it instead of Acanthurus. For the present we follow Cantor and Günther.

${ }^{101}$ This name, commonly used as a synonym of Siganus, has been of ten misspelled as Teuthys, Theutis, etc.

${ }^{402}$ Corrected to Ceris.

403 "Novara Fische," 212; type Acanthurus scopas Cuvier \& Valenciennes. (Omitted in "Genera of Fishes.") 


\section{Family 491. PYGÆID压}

Pyg שus Agassiz, 1838, 191.

Parapygaus Pellegrin, 1907, 526.

Note.-The Plectognathi follow naturally as next in this descent, a fact which can not be shown in a linear series.

\section{Suborder AMPHACANTHI}

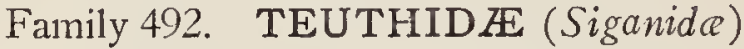

Teuthis ${ }^{404}$ Linnæus, 1766, 23 (in part).

Siganus Forskål, 1775, 32.

Centrogaster Houttuyn, 1782, 44.

Amphacanthus Bloch and Schneider, 1801, 59.
Buro Lacépède, 1803, 67.

Buronus Rafinesque, 1815, 90.

ARCH AEOTEUTH Is ${ }^{408}$ Wettstein, 1886, 436.

Lo Seale, 1906, 519.

The proper application of the name Teuthis is not yet definitely settled. Its first restriction, that of 'Cantor, 1849, makes it identical with Sigamus, but the first use of the name (pre-Linnæus) was for a species of Acanthurus.

${ }^{100}$ Misspelled Archoteuthis in "Genera of Fishes."

\section{Order CATAPHRACTI \\ (Loricati; Parcioplite; Sclerogeni)}

\section{Series SCORPAENIFORMES}

Family 493. SCORPÆNIDÆ

(Scorpion Fishes; Rock Cod)

Scorpæna Linnæus, 1758, 13

Pseudopterus Klein, 1775, 40.

Scorpius (Plumier) Lacépède, 1802, 72.

Tænianotus Lacépède, 1803, 63.

Panotus Rafinesque, 1815,89.

Pterois (Cuvier) Oken, 1817, 104.

AmpHeristus König, 1825, 118.

Apistes ${ }^{408}$ Cuvier, 1829, 127.

Sebastes Cuvier, 1829, 127.

Scorpænopsis Heckel, 1837, 189.

Pteropterus Swainson, 1839, 198.

Trichosomus Swainson, 1839, 197.

Trichophasia Swainson, 1839, 197.

Brachirus Swainson, 1839, 201.

Dendrochirus ${ }^{407}$ Swainson, 1839, 198.

Macrochyrus Swainson, 1839, 201.
Pteroleptus Swainson, 1839, 201.

Pteropterus Swainson, 1839, 201.

Brachyrus ${ }^{408}$ Swainson, 1839, 201.

Platypterus Swainson, 1839, 201.

Pterichthys Swainson, 1839, 201.

Gymnapistes Swainson, 1839, 201.

Goniognathus Agassiz, 1844, 218.

Hypodytes Gistel, 1848, 234.

Corythobatus Cantor, 1849, 241.

Prosopodasys Cantor, 1849, 241.

Scorpænichthys Girard, 1854, 257.

Pteroidichthys Bleeker, 1856, 267.

Scorpænichthys Bleeker, 1856, 267.

Ctenopoma Heckel, 1856, 270.

Scorpænodes Bleeker, 1857, 275.

Polemius Kaup, 1858, 282.

tos Spelled also Apistus.

${ }^{407}$ A synonym of Brachirus, used for the same group on page 71, loc. cit., Dentrochirus occurring on page 180, and Brachyrus on page 264.

${ }^{408}$ Spelled Brachirus by Swainson on page 71, loc. cit., Brachyrus on page 264; replaced by Dendrochirus on page 180, while the name Brachirus is on page 303, taken for a genus of the soles, Synaptura Cantor, of which genus the proper type is Synaptura commersoniana. 
Cocotropus Kaup, 1858, 282.

SCORPANOPTERUS Steindachner, 1859, 293.

Tetraroge Günther, 1860, 296.

Glyptauchen Günther, 1860, 296.

Pentaroge Günther, 1860, 296.

Centropogon Günther, 1860, 296.

Pontinus Poey, 1860, 299.

Sebastodes Gill, 1861, 305.

Sebastichthys Gill, 1862, 317.

Sebastopsis Gill, 1862, 316.

Setarches Johnson, 1863, 319.

Sebastoplus Gill, 1863, 324.

Sebastosomus Gill, 1864, 330.

Sebastomus Gill, 1864, 330.

Neosebastes Guichenot, 1867, 345.

Sebastopsis Sauvage, 1873, 371.

Pseudopterus (Klein) Bleeker, 1876, 384.

Paracentropogon Bleeker, 1876, 384.

Parascorpæna Bleeker, 1876, 384.

Parapterois Bleeker, 1876, 384.

Pteroidichthys Bleeker, 1876, 384.

Amblyapistus Bleeker, 1876, 384.

Cottapistus Bleeker, 1876, 384.

P'seudosebastes Sauvage, 1878, 397.

Eúsebastes Sauvage, 1878, 397.

Sebastapistes Gill, 1878, 397 .

Lioscorpius Günther, 1880, 403.

Sebastolobus Gill, 1881, 415.

Auctospina Eigenmann \& Beeson, 1893 , 460.

Primospina E. \& B., 1893, 460.

Acutomentum E. \& B., 1893, 460.

Pteropodus E. \& B., 1893, 460.

Helicolenus Goode \& Bean, 1895, 468.
Hispanicus Jordan \& Evermann, 1896, 474.

Eosebastes J. \& E., 1896, 474.

Rosicola J. \&. E., 1896, 474.

Emmelas J. \& E., 1898, 481.

Scorpernoides Priem, 1899, 487.

Ectreposebastes Garma11, 1899, 486.

Iracundus Jordan \& Evermann, 1903, 504.

Merinthe Snyder, 1904, 511.

Erisphex Jordan \& Starks, 1904, 509.

Decterias J. \& S., 1904, 509.

Ebosia J. \& S., 1904, 509.

Lythrichthys J. \& S., 1904, 509.

Sebastiscus J. \& S., 1904, 509.

Thysanichthys J. \& S., 1904, 509.

Daia Ogilby, 1904, 510.

Notesthes Ogilby, 1904, 510.

Liocranium Ogilby, 1904, 510.

Plectrogenium Gilbert, 1905, 513.

Sebastosemus Gill, 1905, 513.

Hypomacrus Evermann \& Seale, 1907, 522.

Pogonoscorpius Regan, 1908, 530.

Vespicula ${ }^{40 \theta}$ Jordan \& Richardson, 1910.

Apistops Ogilby, 1911, 540.

Kanekonia Tanaka, 1915, 558.

Sebastella Tanaka, 1918, 565.

Sebastavus Jordan \& Gilbert, 1919, 569.

Rhomarchus Jordan \& Gilbert, 1919, 569.

RixatoR Jordan \& Gilbert, 1920, 571.

Sebastoëssus Jordan, 1920, 571.

Sebastinus Jordan, 1920, 571.

Metzelaaria ${ }^{110}$ Jordan, 1923.

${ }^{400}$ Check List, "Fishes Philippines," 52; orthotype Prosopodasys gogorze Jordan \& Seale. (Omitted in "Genera of Fishes.")

${ }^{10}$ New genus; type Scorpcena tridecimspinosa Metzelaar from Surinam; allied to Auctospina and Pterodus, with thirteen dorsal spines, but with nasal and maxillaty tentacles.

\section{Family 494. CONGIOPODIDE (Agriopida)}

Congiopus ${ }^{411}$ Perry, 1811, 170.

Agriopus Cuvier, 1829, 127.

Cephalinus Gronow, 1854, 259.
Zanclorhynchus Günther, 1880, 403.

Snyderina Jordan \& Starks, 1901, 495.

Ocosia Jordan \& Starks, 1904, 509.

${ }^{111}$ Written Congiopodus by some writers.

\section{Family 495. APLOACTID蚱}

Aploactis Temminck \& Schlegel, 1843, 216.

Sthenopus Richardson, 1848, 240.
Trichopleura Kaup, 1858, 282.

Paraploactis Bleeker, 1865, 335, 384.

Aploactisoma Castelnau, 1872, 363. 


\section{Family 496. SYNANCEJID $\approx$}

Synance ja ${ }^{412}$ Bloch \& Schneider, 1803, 58.

Minous Cuvier \& Valenciennes, 1829, 133.

Pelor Cuvier, 1829, 127.

Spurco (Commerson) Cuvier \& Valenciennes, 1829, 174.

Synanchia ${ }^{413}$ Swainson, 1839, 201.

Erosa Swainson, 1839, 197.

Bufichthys Swainson, 1839, 201.

Trachicephalus Swainson, 1839, 201.

Synancidium Müller, 1843, 216.

Choridactylus ${ }^{414}$ Richardson, 1848, 240.

Glyptauchen ${ }^{415}$ Günther, 1860.

Polycaulus Günther, 1860, 296.

Uranoblepus Gill, 1861, 302.

Synanceichthy s ${ }^{416}$ Bleeker, 1863.
Leptosynanceia Bleeker, 1874, 374.

Synanchia (Swainson) Bleeker, 1874, 374.

Pseudosynanceia Day, 1875, 379.

Emmydrichthys Jordan \& Rutter, 1896, 472.

Choridactylodes Gilchrist, 1902, 498.

Inimicus Jordan \& Starks, 1904, 509.

Erosa (Swainson) Jordan \& Starks, 1904, 509.

Simopias Gill, 1905, 513.

Rhinopias Gill, 1905, 513.

Peloropsis Gilbert, 1905, 513.

Deleastes Seale, 1906, 520.

Lysodermus Smith \& Pope, 1906, 520.

${ }^{112}$ Usually written Synanceia; spelled by Swainson Synanchia.

${ }^{413}$ A variant of Synanceja.

${ }^{114}$ Name later written Chorismodactylus.

${ }^{413}$ "Cat. Fishes," 2: 121; orthotype Apistes panduratus Richardson. (Omitted in "Genera of Fishes.")

110 "Ternate," 11: 324; type Synanceja verrucosa Bloch \& Schneider. (Omitted in "Genera of Fishes.")

\section{Family 497. CARACANTHID正}

Micropus Gray, 1831, 139.

Caracanthus Kröyer, 1845, 227.

Amphiprionichthys Bleeker, 1855, 263.
Crossoderma Guichenot, 1869, 354.

Trachycephalus De Vis, 1884, 427.

\section{Family 498. PAT ECID无}

Patæcus Richardson, 1844, 222.

Neopatæcus Steindachner, 1884, 430.

\section{Family 498a. GNATHANACANTHID开 \\ (Velvet Fishes)}

Gnathanacanthus Bleeker, 1855, 263.

Beridia Castelnau, 1877, 388.

Holoxenus Günther, 1876, 385.

\section{Series HEXAGRAMMIFORMES}

\section{Family 499. ANOPLOPOMID正}

(Skil Fishes; Sable Fishes)

Anoplopoma Ayres, 1858, 277.

Scombrocottus Peters, 1872, 366.
Eoscorpius Jordan \& J. Z. Gilbert, 1919 , 569.

\section{Family 500. ERILEPID王 \\ (Fat-Priests)}

Myriolepis Lockington, 1880, 404.

Ebisus Jordan \& Snyder, 1901, 494.

Erilepis Gill, 1893, 461. 
Family 501. HEXAGRAMMID E

(Greenlings)

Hexagrammos ${ }^{4102}$ (Steller) Tilesius, $1809,76$.

Labrax Pallas, 1810, 77.

Lebius (Steller) Pallas, 1811, 84.

Chirus (Steller) Pallas, 1811, 84.

Chiropsis Girard, 1857, 275, 410.

Agrammus Günther, 1860, 296.
Grammatopleurus Gill, 1861, 305.

Pleurogrammus Gill, 1861, 305.

Acantholebius Gill, 1861, 305.

Octogrammus Bleeker, 1874, 372.

Hexagrammoides Gracianov, 1907, 524.

ACHrestogra M MUS ${ }^{417}$ Jordan, 1921.

ZEMIAGRA M M US ${ }^{418}$ Jordan, 1921.

a10 Usually corrected to Hexagrammus. $\&$ Gilbert.

"17 "Fossil Fishes Cal. Tertiary," 289; orthotype Hexagrammos achrestus Jordan

${ }^{48}$ Loc. cit., 289; orthotype Zemiagrammus isistius Jordan.

Family 502. OPHIODONTIDE

(Cultus Cods)

Ophiodon Girard, 1854, 257.

Oplopoma Girard, 1856, 269.

Family 503. OXYLEBIID $\mathbb{E}$

Oxylebius Gill, 1862, 316.

Family 504. ZANIOLEPID $\mathbb{E}$

Zaniolepis Girard, 1857, 275.

Xantocles Jordan, 1917, 562.

\section{Series PLATYCEPHALIFORMES}

Family 505. HOPLICHTHYIDEE

Hoplichthys ${ }^{410}$ Cuvier \& Valenciennes,

$1829,133$.

${ }^{110}$ Also spelled Oplichthys.

Family 506. PLATYCEPHALID $Æ$

(Flat-heads)

Platycephalus Bloch, 1795, 53.

Calliomorus Lacépède, 1800, 56 .

Centranodon Lacépède, 1803, 66.

Insidiator Jordan \& Snyder, 1900, 490.

Grammoplites Fowler, 1904, 508.

Bembras Cuvier \& Valenciennes, 1829 ,

Bembradium Gilbert, 1905, 513.

133.

Amora $^{420}$ Gray, 1833, 179.

Elates Jordan \& Seale, 1907, 525.

Rogadius Jordan \& Richardson, 1908, 528.

Bambradon J. \& R., 1908, 528.

Neoplatycephalus Castelnau, 1872, 362 .

Hyalorhynchus Ogilby, 1910, 536.

Parabembras Bleeker, 1874, 372.

Thysanophrys Ogilby, 1898, 483.

Inegocia Jordan \& Thompson, 1913, 549.

Onigocia J. \& T., 1913, 549.

${ }^{420}$ Name corrected to Anaora on a plate examined.

\section{Series COTTIFORMES}

Family 507. JORDANIID压

Ventral rays $I, 5$; head narrow; scales present, often reduced each to a few radiating spinules. 
"The scales of Jordania zonope treated with hot caustic potash are nothing but rows of strong ctenoid spines placed as they would be in true scales. In the dorsal region the rows are curved as they would be were they margins of ctenoid scales. In Lepidocottus brevis (Agassiz) the ctenoid elements are as in Jordania, but complete scales are present with circuli and basal radii as usual. It must be supposed that Jordania came from such an ancestor and represents the survival of certain elements of scale structure without the scales." (Cockerell, Science, Dec. 19, 1919, 568.)

Lepidocotrus Sauvage, 1875, 380.

Paricelinus Eigenmann \& Eigenmann, $1889,445$.
Jordania Starks, 1895, 469.

Alcidea Jordan \& Evermann, 1898, 481.

Eocotrus Woodward, 1901, 496.

\section{Family 508. ICELID $\approx$}

Hemilepidotus Cuvier, 1829, 127.

Triglops Reinhardt, 1832, 175.

Temnistia Richardson, 1836, 136.

Enophrys Swainson, 1839, 202.

Icelus Kröyer, 1845, 226.

Aspicottus Girard, 1854, 257.

Clypeocottus Ayres, 1854, 257.

Calycilepidotus Ayres, 1855, 282.

Artedius Girard, 1856, 269.

Ceratocottus Gill, 1859, 290.

Melletes Bean, 1879, 398.

Paraperca Sauvage, $1875,380$.

Chitonotus Lockington, 1881, 416.

Prionistius Bean, 1883, 421.

Icelinus Jordan, 1885, 433.

Artediellus Jordan, 1885, 433.

Radulinus Gilbert, 1890, 451.

Ruscarius Jordan \& Starks, 1895, 469.

Astrolytes J. \& S., 1895, 469.

Rastrinus Jordan \& Evermann, 1896, 474.
Axyrias Starks, 1896, 475.

Tarandichthys Jordan \& Evermann, 1896, 472.

Sternias ${ }^{421}$ J. \& E., 1898.

Stelgistrum Jordan \& Gilbert, 1898, 482.

Archistes J. \& G., 1898, 482.

Stlengis Jordan \& Starks, 1904, 509.

Schmidtia J. \& S., 1904, 509.

Daruma J. \& S., 1904, 509.

Ricuzenius J. \&. S., 1904, 509.

Schmidtina J. \& S., 1904, 510.

Stelgidonotus Jordan \& Thompson, 1905, 513.

Taurulus Gracianov, 1907, 524.

Agonocottus Pavlenko, 1910, 537.

Orthopnias Starks \& Mann, 1911, 541.

Archaulus Gilbert \& Burke, 1912, 543.

Thyriscus Gilbert \& Burke, 1912, 543.

Pterygiocottus ${ }^{422}$ Bean \& Weed, 1920.

422. "Fishes of North and Middle America," II, 1926; orthotype Triglops xenostethus Gilbert. (Omitted in "Genera of Fishes.")

422 "Notes on a Collection of Fishes from Vancouver Island, British Columbia,"

Trans. Roy. Soc. Canada, 13: 73; orthotype P. macouni Bean \& Weed; an ally of Artedius.

\section{Family 509. BLEPSIID $Æ$}

Skin covered with velvety prickles, form compressed.

Blepsias Cuvier, 1829, 127.

Peropus Lay \& Bennett, 1839, 125.

Nautichthys Girard, 1859, 290.
Histiocottus Gill, 1888, 441.

Nautiscus Jordan \& Evermann, 1898, 481.

\section{Family 510. SCORPANICHTHYIDA}

\section{(Cabezones)}

Ventral rays I, 5 ; head broad; skin smooth. Scorpænichthys Girard, 1854, 257. 


\section{Family 511. COTTIDE}

(Sculpins)

Ventral rays $I, 2$, to I, 4; skin naked or with imbedded plates or prickles; general form more or less depressed.

Even after the elimination of several aberrant genera, Jordania, Scorpanichthys, Ascelichthys, Synchirus, Psychrolutes, Blepsias, Abyssocottus, Hemitripterus, Ereunias, Cottocomephorus, Comephorus, Rhamphocottus, etc., the Cottidce are still extremely diversified and may, after study of the skeletons, require further subdivision into families or subfamilies. For purpose of laying stress on distinctions, we further separate certain more or less natural groups, the form centering about Icelus being apparently derived from the ancestors of Jordania, while Cottus, Myoxocephalus and their varied associates may have sprung from ancestors of Scorpanichthys. Both Jordania and Scorpanichthys have normal ventrals (I, 5), but little else in common. Most of the allies of Icelus and Hemilepidotus have developed scales, while none of the Cottidce as here restricted have true scales other than prickles or imbedded dermal plates. But in a general treatment of the group, such a minute subdivision of families may be found superfluous.

Cottus Linnæus, 1758, 12.

Uranoscopus Gronow, 1763, 19.

Myoxocephalus (Steller) Tilesius, 1811, 83.

Pegedictis ${ }^{423}$ Rafinesque, 1820, 113.

Trachydermus Heckel, 1837, 189.

Gymnocanthus ${ }^{424}$ Swainson, 1839, 202.

Uranidea Dekay, 1842, 210.

Centridermichthys Richardson, 1844 , 222.

Phobetor Kröyer, 1845, 226.

Scorpichthes Bonaparte, 1845, 226.

Podabrus Richardson, 1848, 240.

Aphobus Gistel, 1848, 237.

Pseudoblennius Temminck \& Schlegel, $1850,247$.

Pseudoclinus T. \& S., 1850, 247.

Cottopsis Girard, 1851, 249.
Acanthocottus Girard, 1851, 249.

Leptocottus Girard, 1854, 257.

Triglopsis Girard, 1854, 257.

Leiocottus Girard, 1856, 269.

Oligocottus Girard, 1856, 269.

Hoplocottus Kaup, 1858, 282.

Boreocottus Gill, 1859, 290.

Porocottus Gill, 1859, 290.

Ptyonotus Günther, 1860, 296.

Potamocottus Gill, 1861, 307.

Oncocottus Gill, 1861, 302, 313.

Clinocottus Gill, 1861, 305.

Blennicottus Gill, 1861, 305.

Megalocottus Gill, 1861, 305.

Bunocottus $^{425}$ Kner, 1868, 352.

Cottunculus Collett, 1875, 379.

Elaphocottus Sauvage, 1878, 397.

Tauridea Jordan \& Rice, 1878, 395.

${ }^{423}$ Description confused with that of Catonotus.

22 Corrected usually to Gymnacanthus.

${ }^{425}$ This genus, apparently cottoid, is described by Kner as lacking ventral fins, although these structures are plainly shown in his excellent figure. If the description is correct, the genus should stand as a separate family. The fish, Bunocoltus apus, is said to be from deep water, off Cape Horn. But no other cottoid is known south of the Tropic of Cancer. If Kner's account' is correct Bunocottus may be an aberrant scorpænoid. 
Malacocottus Bean, 1890, 450.

Dasycottus Bean, 1890, 450.

Argyrocottus Herzenstein, 1892, 458.

Zesticelus Jordan \& Evermann, 1896, 474.

Oxycottus J. \& E., 1898, 481.

Sigmistes Rutter, 1898, 482.

Dialarchus Greeley, 1901, 493.

Rusciculus Greeley, 1901, 493.

Eximia Greeley, 1901, 493.

Thecopterus H. M. Smith, 1904, 511.

Cottiusculus Schmidt, 1904, 509, 511.

Rheopresbe Jordan \& Starks, 1904, 509.

Ainocottus J. \& S., 1904, 509.

Crossias J. \& S., 1904, 509.
Elaphichthys J. \& S., 1904, 509.

Alcichthys J. \& S., 1904, 509.

Furcina J. \& S., 1904, 509.

Ocynectes J. \& S., 1904, 509.

Bero J. \& S., 1904, 509.

Vellitor J. \& S., 1904, 509.

Cephalocottus Gracianov, 1907, 524.

Mesocottus Gracianov, 1907, 524.

Rusulus Starks \& Mann, 1911, 541.

Trichocottus Soldatov \& Pavlenko, 1915, 557.

Taurocottus S. \& P., 1915, 557.

HAYIA Jordan \& Gilbert, 1919, 569.

Greeleya Jordan, 1920, 571.

\section{Family 512. ABYSSOCOTTID正}

Degenerate sculpins found in the depths of Lake Baikal.

Procottus Gracianov, 1902, 498.

Batrachocottus Berg, 1903, 501.

Abyssocottus Berg, 1906, 516.
Asprocottus Berg, 1906, 516.

Linnocottus Berg, 1906, 516.

Cottinella Berg, 19C7, 521.

\section{Family 513. ASCELICHTHYID压}

Ventral fins wanting; head broad; skins smooth. Ascelichthys Jordan \& Gilbert, 1880, 404.

\section{Family 514. PSYCHROLUTIDAE}

Psychrolutes Günther, 1861, 307.

Gilbertina Jordan \& Starks, 1895, 469.
Gilbertidia Berg, 1899, 484.

Eurymen Gilbert \& Burke, 1912, 543.

\section{Family 515 . NEOPHRYNICHTHYID王}

Until the New Zealand genus Neophrynichthys can be critically examined, it seems best to regard it as constituting a distinct family. As no other cottoid forms have been found south of the Tropic of Cancer, I associate with it provisionally a genus from Buenos Aires.

Neophrynichthys Günther, 1876, 385. Besnardia Lahille, 1913, 549.

Synchirus Bean, 1889, 444.

\section{Family 516. SYNCHIRIDEE}

\section{Family 517. EREUNIID E}

Ereunias Jordan \& Snyder, 1901, 493.

\section{Family 518. RHAMPHOCOTTIDE}

Rhamphocottus Günther, 1874, 375.

\section{Family 519. HEMITRIPTERID 訧}

(Sea Ravens)

Hemitripterus Cuvier, 1829, $117 . \quad$ Ulca Jordan \& Evermann, 1896, 472. 


\section{Family 520. COTTOCOMEPHORID}

Cottocomephorus Pellegrin, 1900, 491. Baicalocottus Berg, 1903, 501.

\section{Family 521. COMEPHORIDE}

(Baical Fishes)

Comephorus Lacépède, 1800, 56.

Elæorhoüs Pallas, 1811, 83.

\section{Family 522. AGONID丑}

(Sea Poachers; Alligator Fishes)

Percis Scopoli, 1777, 41.

Cataphractus Klein, 1777, 43.

Agonus ${ }^{428}$ Bloch \& Schneider, 1801.

Aspidophorus Lacépède, 1802, 62 .

Phalangistes Pallas, 1811, 83.

Cataphractus (Klein) Fleming, 1828, 123.

Hippocephalus Swainson, 1839, 202.

B rachyopsis Gill, 1861, 304, 305.

Agonopsis Gill, 1861, 305.

Leptagonus Giil, 1861, 305.

Hypsagonus Gill, 1861, 305.

Podothecus Gill, 1861, 304.

Paragonus Gill, 1862, 305.

Agonomalus Guichenot, 1866, 341 .

Paragonus Guichenot, 1869, 355.

Siphagonus Steindachner, 1876, 387.

Odontopyxis Lockington, 1879, 400.
Bothragonus (Gill) Jordan \& Gilbert, $1882,420$.

Bathyagonus Gilbert, 1890, 451.

Xenochirus Gilbert, 1890, 451.

Cheiragonus Herzenstein, 1890, 451.

Xystes Jordan \& Starks, 1895, 469.

Averruncus J. \& S., 1895, 469.

Stelgis Cramer, 1895, 469.

Pallasina Cramer, 1895, 469.

Stellerina Cramer, 1896, 474.

Sarritor Cramer, 1896, 474.

Occa Jordan \& Evermann, 1898, 481.

Draciscus Jordan \& Snyder, 1901, 493.

Xeneretmus Gilbert, 1903, 504.

Tilesina Schmidt, 1904, 511.

Asterotheca Gilbert, 1915, 556.

Paragonus Ribeiro, 1918, 564.

Ribeiroa Jordan, 1920, 571.

428 "Systema Ichthyologia," 104; type Cottus cataphractus Linnæus. (Omitted in "Genera of Fishes.")

\section{Family 523. ASPIDOPHOROIDID丑}

Aspidophoroides Lacépède, 1802, 62.

Anoplagonus Gill, 1861, 305.

Canthirhynchus Swainson, 1839, 202.

U1cina ${ }^{427}$ Cramer, 1896.

${ }^{427}$ In Jordan \& Evermann's "Check List," 1896, 449; type Aspidophoroides olriki Lütken. (Omitted in "Genera of Fishes.")

\section{Series CYCLOPTERIFORMES}

\section{Family 524. CYCLOPTERID $\mathbb{E}$}

(Lump Fishes)

Cyclopterus Linnæus, 1758, 12.

Oncotion Klein, 1777, 42.

Lumpus Rafinesque, 1815, 90.

Lumpus Cuvier, 1817, 171.

Family 525. LIPAROPID $\approx$

Aptocyclus De la Pylaie, 1835, 183.

Cyclopterich thys Steindachner, 1881, 417.
Eumicrotremus Gill, 1862, 317, 331.

Cyclopteroides Garman, 1892, 457.

Lethotremus Gilbert, 1895, 469.

Cyclolumpus Tanaka, 1912, 546.

Liparops Garman, 1892, 457. 


\section{Family 526. LIPARID $\mathbb{E}$ (Cyclogasterida)}

(Sea Snails)

Cyclogaster Gronow, 1763, 18.

Liparis Scopoli, 1777, 41.

Liparis Röse, 1793, 52.

Liparius Rafinesque, 1815, 90.

Massaria Gistel, 1848, 236.

Careproctus Kröyer, 1861, 308.

Neoliparis Steindachner, 1875, 381.

Paraliparis Collett, 1878, 393.

Amitra $^{428}$ Goode, 1880, 402.

Monomitra Goode, 1883, 424.

Gymnolycodes Vaillant, 1888, 444.

Enantioliparis Vaillant, 1888, 443.

Bathyphasma Gilbert, 1895, 467.

Gyrinichthys Gilbert, 1895, 467.

Rhinoliparis Gilbert, 1895, 467.

Hilgendorfia Goode \& Bean, 1895, 468.

Amitrichthys ${ }^{423}$ Jordan \& Evermann, 1896, 474.

Allurus J. \& E., 1896, 474.
Allochir J. \& E., 1896, 474.

Caremitra J. \& E., 1896, 474.

Lyoliparis J. \& E., 1896, 474.

Crystallichthys Jordan \& Gilbert, 1898, 482.

Prognurus J. \& G., 1898, 482.

Allinectes Jordan \& Evermann, 1898, 482.

Crystallias Jordan \& Snyder, 1902, 499.

Trismegistus J. \& S., 1904, 508.

Acantholiparis Gilbert \& Burke, 1912, 543.

Elassodiscus G. \& B., 1912, 543.

Polypera Burke, 1912, 542.

Nectoliparis ${ }^{420}$ Gilbert \& Burke, 1912, 543.

Lipariscus Gilbert, 1915, 556.

Cyclogaster (Gronow) Burke, 1912.

A synonym of Paraliparis proper, according to Sigurd Johnsen.

* According to Sigurd Johnsen of Bergen Nectoliparis is a larval Paraliparis. If so, it would seem to be an ally of $P$. holomelas, having a wide gill-slit, and may be generically distinct from Paraliparis, the type of which has a narrow slit as in the group called Amitrichthys.

\section{Series TRIGLIFORMES}

\section{Family 527. PERISTEDIID王}

Peristedion ${ }^{430}$ Lacépède, 1802, 62.

Octonus Rafinesque, 1810, 82.

Peristethus Kaup, 1858, 282.

Vulsiculus Jordan \& Evermann, 1896,
Gargariscus ${ }^{421}$ Smith, 1917.

Heminodus ${ }^{432}$ Smith, 1917.

Scalicus ${ }^{438}$ Jordan, 1922.

475.

${ }^{430}$ Written Peristethus by some authors.

a1 "New Genera of Deep Sea Gurnards (Peristcdiida) from the Philippine Islands," Proc. Biol Soc. Wash., 145; type Gargariscus semidentatus Smith. (Overlooked in "Genera of Fishes.")

42 Smith, op. cit., 146; type Heminodus philippinus Smith.

New genus: type Pcristcdion amiscus Jordan \& Starks; distinguished by the shovel-shaped snout without horns at the angles. Japan. 


\section{Family 528. TRIGLID正}

Trigla Linnæus, 1758, 14.

Corystion Klein, 1775, 41.

Prionotus Lacépède, 1802, 62.

Lepidotrigla Günther, 1860, 296.

Hoplonotus Guichenot, 1866, 341.

Ornichthys Swainson, 1839, 201.

Microtrigla Kaup, 1873, 369.

Chelidonichthys Kaup, 1873, 370.

Palænichthys Kaup, 1873, 370.

Lyrichthys Kaup, 1873, 370.

Trigloides Van Beneden, 1871, 360.
Chriolax Jordan \& Gilbert, 1878, 396.

Podopteryx Sauvage, 1880, 406.

Bellator Jordan \& Evermann, 1896, 475.

Merulinus J. \& E., 1898, 481.

Gurnardus J. \& E., 1898, 481.

Pterygotrigla Waite, 1899, 488.

Colotrigla Gill, 1905, 513.

Fissala Gill, 1905, 513.

Otohime Jordan \& Starks, 1907, 525.

Paratrigla Ogilby, 1911, 540.

Exolissus $^{434}$ Jordan, 1923.

${ }^{434}$ New genus: type Prionotus alepis Alcock, Nat. Hist. Notes, etc., 1889, 303. This genera differs from Prionotus in the absence of scales.

\section{Series DACTYLOPTERIFORMES}

\section{Family 529. DACTYLOPTERIDE (Cephalacanthide)}

Cephalacanthus Lacépède, 1803, 62.

Dactylopterus Lacépède, 180362.

Cephacandia Rafinesque, 1815, 89.

Gonocephalus Gronow, 1854, 258.
Dactyloptena Jordan \& Richardson, 1908, 528.

Daicocus J. \& R., 1908, 528.

Ebisinus J. \& R., 1908, 528.

\section{Order HOLCONOTI}

\section{Family 530. EMBIOTOCID王}

Ditrema Temminck \& Schlegel, 1844, 223.

Embiotoca Agassiz, 1853, 252.

Rhacochilus Agassiz, 1854 (May), 255.

Holconotus Agassiz, 1854 (May), 255.

Amphistichus Agassiz, 1854 (May), 255.

Cymatogaster Gibbons, 1854 (May 18), 257.

Hyperprosopon Gibbons, 1854 (May 18), 257.

Micrometrus Gibbons, 1854 (May 30), 257.

Pachylabrus Gibbons, 1854 (June 21), 257.

Mytilophagus Gibbons, 1854 (July), 257.

Cymatogaster Gibbons, 1854 (June 21 ; not of May 18), 257.

Damalichthys Girard, 1854 (August), 258.

Abeona Girard, 1854, 258.
Ennichthys Girard, 1854, 258.

Phanerodon Girard, 1854, 258.

Metrogaster (Agassiz) Alex. Agassiz, 1861, 300.

Bramopsis (Agassiz), A. Agassiz, 1861, 300.

Tæniotoca A. Agassiz, 1861, 300.

Hypsurus A. Agassiz, 1861, 300.

Brachyistius Gill, 1862, 316.

Hypocritichthys Gill, 1862, 316.

Manichthys (Kaup) Bleeker, 1876, 383.

Sema Jordan, 1878, 396.

Neoditrema Steindachner \& Döderlein, $1883,426$.

Zalembius Jordan \& Evermann, 1896, 473.

Tocichthys Hubbs, 1918, 563.

Amphigonopterus Hubbs, 1918, 563.

\section{Family 531. HYSTEROCARPID \&}

Hysterocarpus Gibbons, 1854, 257.

Dacentrus Jordan, 1878, 396.

Sargosomus (Agassiz) A. Agassiz, 1861, 300 . 


\section{Order CHROMIDES}

\section{Family 532. POMACENTRID 2}

Abudefduf Forskål, 1775, 33 .

Prochilus Klein, 1775, 39.

Amphiprion Bloch \& Schneider, 1801, 59.

Glyphisodon $^{435}$ Lacépède, 1803, 64.

Pomacentrus Lacépède, 1803, 64.

Chromis Cuvier; 1815, 93.

Premnas Cuvier, 1817, 106.

Heliases ${ }^{486}$ Cuvier, 1829, 128.

Dascyllus ${ }^{437}$ Cuvier, 1829, 128.

Pristotis Rüppell, 1835, 184.

Chrysiptera Swainson, 1839, 199.

Odonteus Agassiz, 1839, 194.

Stegastes Jenyns, 1842, 211.

Pirene Gistel, 1848, 235.

Tetradrachmum Cantor, 1849, 241.

Furcaria Poey, 1860, 299.

Hypsypops Gill, 1861, 305.

Lepidozygus Günther, 1862, 318.

Microspathodon Günther, 1862, 318.

Parma Günther, 1862, 318.

Euschistodus Gill, 1862, 315.

Acanthochromis Gill, 1863, 324.

Dischistodus Gill, 1863, 324 .

Ayresia Cooper, 1863, 323.

Pomataprion Gill, 1863, 324.
Onychognathus Troschel, 1866, 343.

Jerdonia Day, 1870, 356.

Paraglyphidodon Bleeker, 1876, 384.

Heptadecacanthus Alleyne \& Macleay, $1877,387$.

Parapomacentrus Bleeker, 1877, 387.

Glyphidodontops Bleeker, 1877, 387.

Brachypomacentrus Bleeker, 1877, 387.

Eupomacentrus Bleeker, 1877, 387.

Amblypomacentrus Bleeker, 1877, 387.

Daya ${ }^{438}$ Bleeker, 1877.

Hemiglyphidodon Bleeker, 1879, 398, 402.

Hermosilla Jenkins \& Evermảnn, 1888, 442.

Nexilarius ${ }^{239}$ Gilbert, 1896, 475.

Azurina Jordan \& McGregor, 1898, 481.

Nexilosus Heller \& Snodgrass, 1903, 503.

Actinicola Fowler, 1904, 508.

Cheiloprion Weber, 1913, 551, 561.

Hoplochromis ${ }^{40}$ Fowler, 1918.

Ctenoglyphiodon ${ }^{411}$ Fowler, 1918.

Azurella Jordan, 1919, 567.

Actinochromis ${ }^{42}$.

Centrochromis ${ }^{433}$ Norman, 1922.

${ }^{45}$ Often written Glyphidodon.

4so Also written Heliastes.

43 Also written Dascillus.

${ }^{43}$ Bleeker, Verh. Holl. Mij. Harlem, 71; orthotype Pomacentrus jerdoni Day. (Omitted in "Genera of Fishes.")

Misprinted Nexilaris.

${ }^{40}$ Proc. Acad. Nat. Sci. Phila., 70:66; orthotype Heliastes caruleus Cuv. \& Val.

${ }^{14}$ Proc. Acad. Nat. Sci. Phila., 1918, 59; type Abudefduf melanopselion Fowler.

"12 Orthotype $A$. lividus. (Further details lacking.)

${ }^{418}$ J. R. Norman, "Fishes from Tobago," Ann. Mag Nat. Hist., 9 : 533; orthotype Glyphidodon rudis Poey.

\section{Family 533. PRISCACARID $巴$}

This extinct group of the American Eocene differs from the Cichlida in the possession of vomerine teeth.

Priscacara Cope, 1877, $388 . \quad$ Cockerellites $^{444}$ Jordan, 1923.

${ }^{44}$ New genus, distinguished from Priscacara by the weak ventral spine and the long soft dorsal of 13 or 14 rays; orthotype Priscacara liops Cope. Named for Theodore Dru Alison Cockerell, of the University of Colorado. 


\section{Family 534. CICHLID丑}

This vast group covers a wide variety of forms, river fishes of both tropics, and doubtless requires subdivision.

Cichla Bloch \& Schneider, 1801, 59.

Chromis Cuvier, 1817, 102.

Etroplus Cuvier \& Valenciennes, 1830, 135.

Cichlaurus Swainson, 1839, 198.

Cichlasoma Swainson, 1839, 200.

Astronotus Swainson, 1839, 200.

Chætolabrus Swainson, 1839, 199.

Microgaster Swainson, 1839, 199.

Tilapia ${ }^{45}$ Andrew Smith, 1840, 244.

Uaru Heckel, 1840, 207.

Symphysod on Heckel, 1840, 207.

Pterophyllum Heckel, 1840, 207.

Acara Heckel, 1840, 207.

Heros Heckel, 1840, 207.

Chætobranchus Heckel, 1840, 207.

Geophagus Heckel, 1840, 207.

Crenicichla Heckel, 1840, 207.

Batrachops Heckel, 1840, 207.

Chætostoma $^{48}$ Heckel, 1846, 230.

Sarotherodon Rüppell, 1852, 251.

Coptodon Gervais, 1853, 253.

Herichthys Baird \& Girard, 1854, 255.

Plataxoides Castelnau, 1855, 264.

Hemichromis Peters, 1857, 276.

Parachromis Agassiz, 1858, 277.

Hypsophrys Agassiz, 1858, 277.

Amphilophus Agassiz, 1858, 277.

Baiodon Agassiz, 1858, 277.

Chromichthys Duméril, 1858, 280.

Haligenes Günther, 1859, 292.

Hoplarchus Kaup, 1860, 297.

Satanoperca Günther, 1862, 318.

Hygrogonus Günther, 1862, 318.

Mesops Günther, 1862, 318.

Mesonauta Günther, 1862, 318.

Petenia Günther, 1862, 318.

Chromis (Cuvier) Günther, 1862, 318.

Theraps Günther, 1862, 318.

Pseudetroplus (Bleeker) Günther, 1862, 318.

Nandopsis Gill, 1862, 315.
Melanogenes Bleeker, 1863, 321.

Neetroplus Günther, 1866, 341.

Paretroplus Bleeker, 1868, 348.

Paratilapia Bleeker, 1868, 348.

Acaropsis Steindachner, 1875, 381.

Crenicara Steindachner, 1875, 381.

Dicrossus (Agassiz) Steindachner, 1875, 381.

Saraca Steindachner, 1875, 381.

Paracara Bleeker, 1878, 392.

Ptychochromis Steindachner, 1880, 406.

Hoplotilapia Steindachner, 1888,443.

Haplochromis Hilgendorf, 1888, 442.

Oreochromis Günther, 1889, 446.

Lamprologus Schilthuis, 1890, 452.

Ctenochromis ${ }^{47}$ Pfeffer, 1893.

Retroculus Eigenmann \& Bray, 1894, 464.

Æquidens Eigenmann \& Bray, 1894, 464.

Pelmatochromis Steindachner, 1894, 465.

Docimodus Boulenger, 1896, 471.

Corematodus Boulenger, 1896, 471.

Boggiania Perugia, 1897, 478.

Chromidotilapia Boulenger, 1898, 479.

Telmatochromis Boulenger, 1898, 479.

Julidochromis Boulenger, 1898, 479.

Eretmodus Boulenger, 1898, 479.

Bathybates Boulenger, 1898, 479.

Tropheus Boulenger, 1898, 479.

Petrochromis Boulenger, 1898, 479.

Simochromis Boulenger, 1898, 479.

Perissod us Boulenger, 1898, 479.

Plecodus Boulenger, 1898, 479.

Ectodus Boulenger, 1898, 576.

Xenochromis Boulenger, 1899, 485.

Xenotilapia Boulenger, 1899, 485.

Trematocara Boulenger, 1899, 485.

Steatocranus Boulenger, 1899, 485.

Grammatotria Boulenger, 1899, 485.

Pseudoplesiops Boulenger, 1899, 485.

Spathodus Boulenger, 1900, 488.

Asprotilapia Boulenger, 1901, 492.

Gephyrochromis Boulenger, 1901, 492.

\footnotetext{
${ }^{46}$ Type Tilapia sparrmanni Smith.

${ }^{40}$ Written Chatostomus by Kner, 1854.

${ }^{47}$ Type Ctenochromis pectoralis, a synonym of Haplochromis. (Omitted in

"Genera of Fishes" and in Zoological Record.)
} 
Hemitilapia Boulenger, 1902, 497.

Cyrtocara Boulenger, 1902, 497.

Chilochromis Boulenger, 1902, 497.

Biotæecus Eigenmann \& Kennedy, 1903, 502.

Biotodoma Eigenmann \& Kennedy, 1903, 502.

Ophthalmotilapia Pellegrin, 1904, 510.

Boulengerochromis Pellegrin, 1904, 510.

Astatotilapia Pellegrin, 1904, 510.

Nannochromis Pellegrin, 1904, 510.

Astatoreochromis Pellegrin, 1904, 510.

Lepidolamprologus Pellegrin, 1904, 510.

Astatheros Pellegrin, 1904, 510.

Thorichthys Meek, 1904, 510.

Nannacara Regan, 1905, 515.

Parapetenia Regan, 1905, 515.

Astatochromis Pellegrin, 1905, 515.

Platytæniodus Boulenger, 1906, 517.

Haplotaxodon Boulenger, 1906, 517.

Cunningtonia Boulenger, 1906, 517.

Heterogramma Regan, 1906, 520.

Enantiopus Boulenger, 1906. (See Index.)

Chætobranchopsis Eigenmann \& Ward, 1907, 522.

Erythrichthys Meek, 1907, 525.

Palsochromis Sauvage, 1907, 526, 537.

Tomocichla Regan, 1908, 530.

Chilotilapia Boulenger, 1908, 527.

Bayonia Boulenger, 1911, 538.

Acarichthys Eigenmann, 1912, 543.

Stappersia Boulenger, 1914, 552.

Schubotzia Boulenger, 1914, 552.
Champsochromis ${ }^{48}$ Boulenger, 1916, 558.

Lobochilotes Boulenger, 1916, 558.

Gymnogeophagus Ribeiro, 1918, 565.

Nannacara Ribeiro, 1918, 564.

Pseudopercis Ribeiro, 1918, 564.

Otopharynx Regan, 1920, 572.

Limnotilapia Regan, 1920, 572.

Cyathopharynx Regan, 1920, 572.

Limnochromis Regan, 1920, 572.

Cyohotilapia Regan, 1920, 572.

Lipochromis Regan, 1920, 572.

Neochromis Regan, 1920, 572.

Cnestrostoma Regan, 1920, 572.

Mylochromis Regan, 1920, 572.

Sargochromis Regan, 1920, 572.

Labrochromis Regan, 1920, 572.

Serranochromis Regan, 1920, 572.

Clinodon Regan, 1920,572.

Callochromis Regan, 1920, 572.

Leptochromis Regan, 1920, 572.

Aulonocranus Regan, 1920, 572.

Hemibates Regan, 1920, 572.

Neotilapia Regan, 1920, 572.

Heterotilapia Regan, 1920, 572.

Tylochromis Regan, 1920, 572.

Pseudotropheus ${ }^{48}$ Regan, 1921.

Cynotilapia ${ }^{400}$ Regan, 1921.

Lethrinops ${ }^{451}$ Regan, 1921.

Rhamphochromis ${ }^{452}$ Regan, 1921.

Aulonocara ${ }^{453}$ Regan, 1921.

Heterochromis ${ }^{4582}$ Regan, 1922.

Parachromis ${ }^{436 \mathrm{~b}}$ Regan, 1922.

Macropleurodus Regan, 1922. (See Index.)

${ }^{448}$ A synonym of Haplochromis.

448 "Cichloid Fishes of Lake Nyassa," Proc. Zool. Soc. Lond., 681; orthotype Chromis williamsi Günther.

${ }^{450}$ Loc. cit., 684 ; orthotype C. afra Günther.

${ }^{451}$ Loc. cit., 718; orthotype Chromis lethrinus Günther.

${ }^{452}$ Loc. cit.; orthotype Hemichromis longiceps Günther.

${ }^{163}$ Loc. cit.; orthotype $A$. nyasse Regan.

435a "Classification of the Fishes of the Family Cichlide II"; African and Syrian Genera, Ann. Mag. Nat. Hist. X., September, 1922, 252; orthotype Paratilapia multidens Pellegrin.

${ }^{4531}$ Loc. cit., 251 ; orthotype Hemichromis sacer Günther. 


\section{Order PHARYNGOGNATHI}

\section{Family 535. PHARYNGOPILID E (Phyllodontida)}

A small extinct family of Labroid fishes, with the upper pharyngeals united into one, the median teeth enlarged.

Phyllodus Agassiz, 1844, 217.

LABRoDON Gervais, 1857, 275.

Num mopalatus Rouault, 1858, 284.

Diaphyodus Schaffhäut1, 1863, 328.
Pharyngopilus Cocchi, 1864, 329.

Egertonia Cocchi, 1864, 329.

PARAPHyllodus ${ }^{454}$ Sauvage, 1875.

${ }^{454}$ Bull. Geol. Soc. France, 3: 615; name given to species of Phvllodus with the median teeth small. (Omitted in "Genera of Fishes.")

\section{Family 536. LABRID $\mathbb{E}$}

This family may be naturally separated into the tropical forms, having the vertebræ in normal number $(10+15=25)$, and those with the number increased $(10+17=27$ to $20+21=41)$. Those with the increased numbers of vertebræ inhabit the colder seas.

Labrus Linnæus, 1758, 13.

Scarus Gronow, 1763, 19.

Suillus Catesby, 1771, 30.

Cicla Klein, 1775, 39.

Helops Browne, 1789, 47.

Bodianus ${ }^{45}$ Bloch, 1790, 49.

Cicla Röse, 1793, 52.

Hiatula Lacépède, 1800, 57.

Harpe Lacépède, 1803, 63.

Symphodus Rafinesque, 1810, 79.

Tautoga Mitchill, 1814, 86.

Corycus $^{\text {ss }}$ Cuvier, 1815, 93.

Crenilabrus Cuvier, 1815, 93.

Diastodon Bowdick, 1825, 119.

Lachnolaimus ${ }^{457}$ Cuvier, 1829, 130.

Clepticus Cuvier, 1829, 130.

Ctenolabrus Cuvier \& Valenciennes, 1839, 194.

Acantholabrus C. \& V., 1839, 194.

Cossyphus C. \& V., 1839, 194.

Malapterus ${ }^{48}$ C. \& V., 1839.

Cynædus Swainson, 1839, 200.

Chcerodon Bleeker; 1847, 230.
Ronchifex Gistel, 1848, 235.

Platylamus Dixon, 1850, 245.

Chœrops Rüppell, 1852, 251.

Pteragogus Peters, 1855, 266.

Xiphocheilus Bleeker, 1856, 268.

Cossyphodes Bleeker, 1860, 293.

Pseudodax $^{458}$ Bleeker, 1861.

Hypsigenys Günther, 1861, 308.

Semicossyphus Günther, 1861, 308.

Tautogolabrus Günther, 1862, 318.

Trochocopus Günther, 1862, 318.

Decodon Günther, 1862, 318.

Lepidaplois Gill, 1862, 314, 325.

Euhypsocara Gill, 1863, 325.

Gymnopropoma Gill, 1863, 325.

Achœrodus Gill, 1863, 325.

Pimelometopon Gill, 1864, 330.

Heterochœrops Steindachner, 1866, 343.

Phacodus Cope, 1869, 354.

Crommyodus Cope, 1870, 356.

Protautoga Leidy, 1873, 370.

Torresia Castelnau, 1875, 378.

Platychœrops Klunzinger, 1880, 404.

\footnotetext{
${ }^{4 s 5} \mathrm{By}$ tautonomy, not by first restriction.

${ }^{456}$ Later written Coricus.

${ }^{467}$ Corrected later to Lachnolomus.

${ }^{4}$ Poissons, 12 : 355; type $M$. reticulatus Cuvier \& Valenciennes; spelled Malacopterus by Günther. (Omitted in "Genera of Fishes.")

${ }^{\star \infty}$ Bleeker, "Scaroidei," 2; orthotype Odax moluccensis Cuvier \& Valenciennes. (Omitted in "Genera of Fishes.")
} 
Stylodus Sauvage, 1882, 421.

Pseudosph TrRodon Noetling, 1885, 434.

Graus Philippi, 1887, 438.

Lappanella Jordan, 1890, 452.

Protautoga di Alessandri, 1896, 471.

Teleogramma Boulenger, 1899, 453.

Verreo Jordan \& Snyder, 1902, 499.

\section{Family 537. CORIDÆ}

Platiglossus Klein, 1775, 40.

G'omphosus Lacépède, 1802, 61.

Coris Lacépède, 1802, 61.

Hemipteronotus Lacépède, 1802, 61.

Acarauna Sewastianof, 1802, 68.

Elops (Commerson) Lacépède, 1802, 70.

Hologymnosus Lacépède, 1802, 63.

Cheilinus Lacépède, 1802, 63.

Cheilio Lacépède, 1803, 63.

Tautoga Mitchill, 1814, 86.

Micropodus Rafinesque, 1815, 89.

Xyrichthys ${ }^{400}$ Cuvier, 1815, 93.

Epibulus Cuvier, 1815, 93.

Aygula Rafinesque, 1815, 90.

Julis Cuvier, 1815, 94.

Novacula Cuvier, 1817, 102.

Anampses (Cuvier) Quoy \& Gaimard, 1824, 117.

Amorphocephalus Bowdich, 1825, 119.

Elops (Commerson) Bonaparte, 1831, 175.

Halichœres Rüppell, 1835, 184.

Thalassoma Swainson, 1839, 199.

Crassilabrus Swainson, 1839, 199.

Urichthys Swainson, 1839, 199.

Ichthycallus Swainson, 1839, 200.

Eupemis Swainson, 1839, 200.

Chlorichthys Swainson, 1839, 200.

Thalliurus Swainson, 1839, 200.

Cirrhilabrus Temminck \& Schlegel, 1845, 227.

Neanis Gistel, 1848, 236.

Cheilinoides Bleeker, 1851, 248.

Labroides Bleeker, 1851, 248.
Verriculus Jordan \& Evermann, 1903, 504.

Chœropsodes Gilchrist \& Thompson, 1909, 533.

Gillidia Eastman, 1914, 553.

Eolabroides Eastman, 1914, 552.

Labrichthys Bleeker, 1854, 256.

Duymæria Bleeker, 1856, 267.

Xiphocheilus $^{481}$ Bleeker, 1856, 268.

Labrastrum Guichenot, 1860, 296.

Stethojulis Günther, 1861, 308.

Hemigymnus Günther, 1861, 308.

Doratonotus Günther, 1862, 318.

Cymolutes Günther, 1862, 318.

Ophthalmolepis ${ }^{42}$ Bleeker, 1862, 300.

Macropharyngodon Bleeker, 1862, 309.

Platyglossus (Klein) Bleeker, 1862, 309.

Hemicoris Bleeker, 1862, 309.

Pseudojulis Bleeker,1862, 309.

Pseudocheilinus Bleeker, 1862, 309.

Pseudocoris Bleeker, 1862, 309.

Guntheria Bleeker, 1862, 309.

Pseudolabrus Bleeker, 1862, 309.

Hemitautoga Bleeker, 1862, 309.

Diproctacanthus Bleeker, 1862, 309.

Novaculichthys Bleeker, 1862, 309.

Leptojulis Bleeker, 1862, 309.

Oxycheilinus $^{483}$ Gill, 1862, 315.

Malacocentrus Gill, 1862, 314.

Chœrojulis Gill, 1862, 314.

Iniistius Gill, 1862, 314.

Dimalacocentrus Gill, 1863, 325.

Oxyjulis Gill, 1863, 325.

Cheiliopsis Steindachner, 1863, 328

Thysanocheilus Kner, 1865, 338.

Parajulis Bleeker, 1865, 335.

Cheilolabrus Alleyne \& Macleay, 1877, 387.

Austrolabrus Steindachner, 1884, 430.

${ }^{400}$ Spelled also Xirichthys.

"er Corrected to Xiphochilus.

${ }^{422}$ The date of this paper (No. 712) is 1862, and the genera which follow are included in it as well as in No. 754. The type of Hemicoris is Halichoeres variegatus Rüppell; that of Guntheria, G. caruleovittatus Bleeker; that of Hemitautoga, Labrus centiquadrus Lacépède; and of Novaculichthys, Labrus taniourus Lacépède.

${ }^{*}$ Orthotype Cheilimus arenatus. 
Julichthys De Vis, 1885, 431.

Eupetrichthys Ramsey \& Ogilby, 1887, 439.

Xyrula Jordan, $1890,452$.

Pictilabrus Gill, 1891, 455.

Julidio Jordan \& Evermann, 1896, 473.

Emmeekia J. \& E., 1896, 473.
Iridio J. \&. E., 1896, 473.

Ampheces Jordan \& Snyder, 1902, 499.

Octocynodon Fowler, 1904, 508.

Chœropsodes Gilchrist \& Thompson, 1909, 533.

Bermudichthys Nichols, 1920, 572.

Hinalea ${ }^{484}$ Jordan \& Jordan, 1923.

${ }^{464}$ "Fishes of Hawaii," Mem. Carnegie Mus. 69; orthotype Julis axillaris Quoy and Gaimard; differs from Stethojulis in lacking posterior canines.

\section{Family 538. NEOLABRID $\mathbb{E}$}

Allied to the Coridce, but with the weak dorsal spines only 3 or 4 in number.

Neolabrus Steindachner, 1875, 381.

\section{Family 539. SPARISOMIDE (Scarichthyida)}

This group is well distinguished from the Scarida, by its very different pharyngeal dentition as well as by numerous other characters of fins and teeth.

Leptoscarus Swainson, 1839, 199.

Sparisoma Swainson, 1839, 199.

Scarus (Forskål) Bleeker, 1849, 240.

Scarichthys Bleeker, 1859, 287.

Taurinichthys Cocchi, 1864, 329.

Cryptotomus Cope, 1871, 361.
Heteroscarus Castelnau, 1872, 363.

Calotomus Gilbert, 1890, 451.

Scaroides Tould, 1899, 534.

Scaridea Jenkins, 1901, 493.

Xenoscarus Evermann \& Radcliffe, 1917, 562.

\section{Family 540. SCARID $F$ (Callyodontida)}

Callyodon Gronow, 1763, 19.

Psittacus Catesby, 1771, 31.

Novacula Catesby, 1771, 31.

Scarus ${ }^{485}$ Forskål, 1775, 33.

Callyodon $^{486}$ (Gronow) Scopoli, 1777, 42.

Callyodon (Gronow) Bloch, 1788, 45.

Mormyra Browne, 1789, 46.

Calliodon Bloch \& Schneider, 1801, 59.

Odax (Commerson) Lacépède, 1802, 70.
Aper (Plumier) Lacépède, 1803, 72.

Calliodon Cuvier, 1829, 130.

Hemistoma Swainson, 1839, 199.

Petronason Swainson, 1839, 199.

Erychthys Swainson, 1839, 199.

Chlorurus Swainson, 1839, 199.

Amphiscarus Swainson, 1839, 199.

Euscarus Jordan \& Evermann, 1896, 473.

Loro J. \& E., 1896, 473.

${ }^{405} \mathrm{I}$ use this familiar name pending final decision as to the retention of Gronow's non-binomial post-Linnæan generic names.

${ }^{468}$ Also often written Calliodon.

\section{Family 541. ODACID $\mathbb{E}$}

Odax Cuvier, 1829, 130.

Olisthops ${ }^{487}$ Richardson, 1850, 247.

Coridodax Günther, 1862, 318.
Heteroscarus Castelnau, 1872, 363.

Neoodax Castelnau, 1875, 378.

${ }^{107}$ Spelled Olistherops by Günther.

\section{Family 542. SIPHONOGNATHIDE}

Siphonognathus Richardson, 1857, 276. 


\section{Order GOBIOIDEA}

\section{Family 543. RHYACICHTHYID王 (Platypterida)}

Platyptera (Kuhl \& Van Hasselt)

Cuvier, 1829, 1300, 189.

\section{Family 544. ELEOTRID丑}

(Sleepers)

Eleotris Gronow, 1763, 20.

Pelmatia Browne, 1789, 47.

Gobiomorus Lacépède, 1800, 57.

Gobiomoroides Lacépède, 1800, 57.

Eleotris Bloch \& Schneider, 1801, 58.

Bostrychus Lacépède, 1802, 61.

Bostrychoides Lacépède, 1802, 61 .

Asellus (Plumier) Lacépède, 1802, 71.

Bostrichthys Duméril, 1806, 75.

Bostrictis Rafinesque, 1815, 88.

Psilus Fischer, 1813, 85.

Psiloides Fischer, 1813, 85.

Pterops Rafinesque, 1815, 88.

Ictiopogon Rafinesque, 1815, 92.

Epiphthalmus Rafinesque, 1815, 90.

Prochilus Cuvier, 1817, 104.

Asterropteryx Rüppell, 1828, 122.

Philypnus Cuvier \& Valenciennes, 1837, 188.

Cestreus McClelland, 1842, 212.

Culius Bleeker, 1856, 267, 372.

Butis Bleeker, 1856, 267, 372.

Belobranchus Bleeker, 1856, 268, 372 .

Eleotriodes Bleeker, 1857, 275, 373.

Lembus Günther, 1859, 291.

Erotelis Paey, 1860, 299.

Dormitator Gill, 1861, 302, 316.

Mogurnda Gill, 1863, 326.

Gobiomorphus Gill, 1863, 326.

Ophiocara Gill, 1863, 326.

Hypseleotris Gill, 1863, 326.

Odonteleotris Gill, 1863, 326.
Calleleotris Gill, 1863, 326.

Ptereleotris Gill, 1863, 326.

Valenciennea Bleeker, 1868, 348.

Valenciennesia Bleeker, 1874, 373 .

Amblyeleotris Bleeker, 1874, 372 .

Eleotrioides Bleeker, 1874, 372.

Guavina Bleeker, 1874, 372.

Oxyeleotris Bleeker, 1874, 372.

Gymneleotris Bleeker, 1874, 372.

Gymnobutis Bleeker, 1874, 372.

Prionobutis Bleeker, 1874, 372 .

Odontobutis Bleeker, 1874, 372.

Priolepis (Ehrenberg) Bleeker, 1874, 373.

Brachyeleotris Bleeker, 1874, 373.

Hetereleotris Bleeker, 1874, 373.

Pogoneleotris Bleeker, 1875, 377.

Giuris Sauvage, 1879, 401.

Ioglossus Bean, 1882, 419, 420.

Alexurus Jordan, 1895, 468.

Krefftius Ogilby, 1897, 478.

Carassiops Ogilby, 1897, 477.

Mulgoa Ogilby, 1897, 478.

Ophiorrhinus Ogilby, 1897, 478.

Caulichthys Ogilby, 1898, 483.

Vireosa Jordan \& Snyder, 1901, 494.

Eviota Jenkins, 1903, 504.

Parioglossus ${ }^{488}$ Regan, 1912, 545.

Microeleotris Meek \& Hildebrand, 1916, 559.

Hemieleotris M. \& H., 1916, 560.

Leptophilypnus M. \& H., 1916, 559.

${ }^{468}$ Misprinted Pariglossus by authors.

Family 545. GOBIID无

(Gobies)

Gobius Linnæus, 1758, 12.

Gobio Klein, 1779, 43.

Benthophilus Eichwald, 1831, 176.

Oplopomus (Ehrenberg) Cuvier \& Valenciennes, 1837, 188.

Cryptocentrus (Ehrenberg) Cuvier \& Valenciennes, 1837, 188, 374.
Apocryptes Cuvier \& Valenciennes, 1837, 188.

Hexacanthus Nordmann, 1838, 193.

Ruppellia Swainson, 1839, 202.

Gobileptes Swainson, 1839, 198.

Brachyochirus Nardo, 1841, 209.

Chæturichthys Richardson, 1844, 222. 
Gobiodon (Kuhl \& Van Hasselt) Bleeker, 1856, 267.

Oxyurichthys Bleeker, 1858, 279.

Lepidogobius Gill, 1858, 280.

Tridentiger Gill, 1858, 280.

Triænophorus Gill, 1858, 280.

Triænophorichthys Gill, 1858, 280.

Chænogobius Gill, 1858, 280.

Ctenogobius Gill, 1858, 281.

Gobionellus Girard, 1859, 291.

Gobiosoma Girard, 1859, 291.

Euctenogobius Gill, 1859, 291.

Evorthodus Gil1, 1859, 289.

Acanthogobius Gill, 1859, 290.

Glossogobius Gi1l, 1859, 290.

Rhinogobius Gill, 1859, 290.

Luciogobius Gill, 1859, 290.

Smaragdus Poey, 1860, 299.

Chonophorus Poey, 1860, 299.

Awaous Steindachner, 1860, 299.

Cyclogobius Steindachner, 1860, 299.

Oplopomus Steindachner, 1860, 299.

Gobiopsis Steindachner, 1860, 299.

Oxyurichthys Bleeker, 1860, 294.

Latrunculus Günther, 1861, 307.

Lentipes Günther, 1861, 307.

Lophogobius Gill, 1862, 316.

Eucyclogobius Gill, 1862, 316.

Gillichthys Cooper, 1863, 323.

Boreogobius Gill, 1863, 326.

Crystallogobius Gill, 1863, 326.

Gymnogobius Gill, 1863, 326.

Ophiogobius Gill, 1863, 326.

Synechogobius Gill, 1863, 326.

Pterogobius Gill, 1863, 326.

Coryphopterus Gill, 1863, 325.

Deltentosteus Gill, 1863, 325.

Pomatoschistus Gill, 1863, 325.

Cotylopus Guichenot, 1864, 332 .

Gillia Günther, 1864, 334.

Orthostomus Kner, 1868, 352.

Gobiichthys Klunzinger, $1871,361$.

Paragobiodon Bleeker, 1873, 367.

Lophiogobius Günther, 1873, 369.

Ellerya Castelnau, 1873, 367.

Alepidogobius Bleeker, 1874, 373.

Gobiopterus Bleeker, 1874, 373.

Leptogobius Bleeker, 1874, 373.

Triænopogon Bleeker, 1874, 373.
Sicydiops Bleeker, 1874, 373.

Microsicydium Bleeker, 1874, 373.

Brachygobius Bleeker, 1874, 373.

Callogobius Bleeker, 1874, 373.

Platygobius Bleeker, 1874, 373.

Mesogobius Bleeker, 1874, 373.

Stenogobius Bleeker, 1874, 373.

Hemigobius Bleeker, 1874, 373.

Oligolepis Bleeker, 1874, 373 .

Gnatholepis Bleeker, 1874, 373.

Hypogymnogobius Bleeker, 1874, 373.

Actinogobius Bleeker, 1874, 373.

Cephalogobius Bleeker, 1874, 373.

Centrogobius Bleeker, 1874, 373.

Acentrogobius Bleeker, 1874, 373.

Porogobius Bleeker, 1874, 373.

Pseudogobiodon Bleeker, 1874, 373.

Oxymetopon Bleeker, 1874, 373.

Paragobiodon Bleeker, 1874, 373.

Amblygobius Bleeker, 1874, 373.

Zonogobius Bleeker, 1874, 374.

Odontogobius Bleeker, 1874, 374.

Stigma togobius Bleeker, 1874, 374.

Amblychæturichthys Bleeker, 1874, 374.

Parachæturichthys Bleeker, 1874, 374.

Apocryptodon Bleeker, 1874, 374.

Parapocrytes Bleeker, 1874, 374.

Gobileptes (Swainson) Bleeker, 1874, 374.

Pseudapocryptes Bleeker, 1874, 374.

Orthostomus Kner, 1874, 373.

Gobiopus Gill, 1874, 375.

Microgobius Poey, 1875, 379.

Latrunculodes Collett, 1875, 379.

Leptogobius Bleeker, 1875, 377.

Apocryptichthys Day, 1876, 385.

Paroxyurichthys Bleeker, 1876, 384.

Lebetus Winther, 1877, 392.

Perccottus Dybowski, 1877, 389.

Bathygobius ${ }^{489}$ Bleeker, 1878, 393.

Typhlogobius Steindachner, 1880, 406.

Leucopsarion Hilgendorf, 1880, 403.

Othonops Rosa Smith (Eigenmann), 1881, 417.

Saccostoma Sauvage, 1882, 421.

Leme De Vis, 1884, 427.

Clevelandia Eigenmann \& Eigenmann, $1888,440$.

Barbulifer E. \&. E., 1888, 440.

\footnotetext{
${ }^{460}$ An unfortunate name for a very common shallow-water form.
} 
Bollmannia Jordan, 1889, 447.

Chriolepis Gilbert, 1891, 455.

Salarigobius Pfeffer, 1893, 462.

Garmannia Jordan, 1895, 576.

Quietula Jordan \& Evermann, 1895, 469.

Aboma Jordan \& Starks, 1895, 468.

Evermannia Jordan, 1895, 468.

Stiphodon ${ }^{400}$ Weber, 1895, 470.

Sicya Jordan \& Evermann, 1896, 474.

Zalypnus J. \& E., 1896, 474.

Lythrypnus J. \& E., 1896, 474.

Ilypnus J. \& E., 1896, 474.

Sicyosus J. \& E., 1898, 482.

Enypnias J. \& E., 1898, 481.

Trichopharynx Ogilby, 1898, 483.

Austrogobio Ogilby, 1898, 483.

Oreogobius Boulenger, 1899, 485.

Mapo Smitt, 1899, 487.

Lebistes Smitt, 1899, 487.

Caffrogobius Smitt, 1899, 487.

Mugilogobius Smitt, 1899, 487.

Proterorhinus Smitt, 1899, 487.

Eichwaldia Smitt, 1899, 487.

Trifissus Jordan \& Snyder, 1900, 490.

Chasmias J. \& S., 1901, 494.

Eutæniichthys J. \& S., 1901, 494.

Clariger J. \& S., 1901, 494.

Astrabe J. \& S., 1901, 494.

Ainosus J. \& S., 1901, 494.

Sagamia J. \& S., 1901, 494.

Suruga J. \& S., 1901, 494.

Chloëa J. \& S., 1901, 494.

Hazeus J. \& S., 1901, 494.

Chasmichthys J. \& S., 1901, 494, 504.

Mistichthys H. M. Smith, 1902, 500.

Chlamydes Jenkins, 1903, 504.

Vitraria Jordan \& Evermann, 1903, 504.

Quisquilius J. \& E., 1903, 504.
Elacatinus Jordan, 1904, 508.

Pycnomma Rutter, 1904, 511.

Allogobius Waite, 1904, 511.

Gigantogobius Fowler, 1905, 513.

Kelloggella Jordan \& Seale, 1905, 514, 519.

Drombus J. \& S., 1905, 514.

Waitea J. \& S., 1906, 519.

Pselaphias J. \& S., 1906, 519.

Exyrias J. \& S., 1906, 519.

Mars J. \& S., 1906, 519.

Vailima J. \& S., 1906, 519.

Vaimosa J. \& S., 1906, 519.

Vitreola J. \& S., 1906, 519.

Trimma J. \& S., 1906, 519.

Caragobius Smith \& Seale, 1906, 520.

Illana S. \& S., 1906, 520.

Ranulina Jordan \& Starks, 1906, 519.

Creisson Jordan \& Seale, 1907, 525.

Xenisthmus Snyder, 1908, 531.

Doryptena Snyder, 1908, 531.

Aparrius Jordan \& Richardson, 1908, 528.

Macgregorella Seale, 1909, 533.

Biat Seale, 1909, 533.

Expedio Snyder, 1909, 534.

Inu Snyder, 1909, 534.

Nematogobius Boulenger, 1910, 534.

Mucogobius McCulloch, 1912, 544.

Schismatogobius Beaufort, 1912, 542.

Pleurosicya Weber, 1913, 551.

Lubricogobius Tanaka, 1915, 558.

Evermannichthys ${ }^{471}$ Metzelaar, 1920.

Xenogobius ${ }^{42}$ Metzelaar, 1920.

Micropercops $^{43}$ Fowler \& Gee, 1920.

U1cigobius ${ }^{44}$ Fowler, 1920.

Aprolepis ${ }^{45}$ Hubbs, 1921.

Radcliffella ${ }^{476}$ Hubbs, 1921.

${ }^{470}$ The reference on page 542 in "Genera of Fishes" should be canceled.

${ }^{471}$ Over Tropisch Atlantisch Visschen, 139; type Evermannichthys spongicola

Metzelaar.

${ }^{472}$ Op. cit., 140; type Xenogobius weberi Metzelaar.

${ }^{473}$ Proc. U. S. Nat. Mus., 58: 318; type Micropercops dabryi Fowler \& Bean.

${ }^{474}$ Proc. Acad. Nat. Sci. Phila., 1918, 69: type Drombus maculipinnis Fowler.

${ }^{475}$ Occ. Papers Univ. Michigan, 99 : 1; orthotype Aprolepis barbara Hubbs.

${ }^{476}$ Occ. Papers Univ. Mich., $99: 2$; orthotype Garmannia spongicola Radcliffe. 
Family 546. PERIOPHTHALMID王

Apocryptes ${ }^{47}$ Osbeck, 1762, 17.

Periophthalmus Bloch \& Schneider, $1801,58$.

Scartelaos Swainson, 1839, 202.

Apocryptes (Osbeck) Cuvier \& Valenciennes, 1837, 188.
Boleophthalmus C. \& V., 1837, 188.

Euchoristopus Gill, 1863, 326.

Boleops Gill, 1863, 326.

Periophthalmodon Bleeker, 1874, 374.

${ }^{477}$ A binomial name, but first issued in 1757 , before the "Systema Naturæ" of Linnæus; reprinted in 1762 . The name was revived by Cuvier \& Valenciennes, its designated type being that of Osbeck, identical with Bolcophthalmus, described on a subsequent page of the "Histoire Naturelle des Poissons." The name Apocryptes was later transferred to a different group.

\section{Family 547. GOBIOIDID $\mathbb{E}$}

Gobioides Lacépède, 1800, 57.

Tænioides Lacépède, $180 C, 57$.

Plecopodus Rafinesque, 1815, 90.

Gymnurus Rafinesque, $1815,88$.

Amblyopus Cuvier \& Valenciennes, 1837, 188.

Psilosomus Swainson, 1839, 198.
Ognichodes Swainson, 1839, 202.

Tyntlastes Günther, 1862, 319.

Brachyamblyopus Bileeker, 1874, 374.

Odontamblyopus Bleeker, 1874, 374.

Cayennia Sauvage, 1880, 405.

Paragobioides Kendall \& Goldsborough, 1911, 540 .

\section{Family 548. TRYPAUCHENID $Æ$}

Trypauchen $^{478}$ Cuvier \& Valenciennes, Ctenotrypauchen Steindachner, 1867, 1837.

Trypauchenichthys Bleeker, 1860, 293, Trypauchenopsis Volz, 1903, 506. 374.

Trypauchenophrys Franz, 1910, 535.

${ }^{478}$ "Histoire Naturelle des Poissons," XII, 162; type Gobius vagina Bloch \& Schneider. (Omitted in "Genera of Fishes.")

\section{Family 549. DOLIICHTHYID E}

Doliichthys Sauvage, 1874, 376.

\section{Family 550. PSAMMICHTHYIDÆ}

Psammichthys Regan, 1908, 530.

\section{Order DISCOCEPHALI}

\section{Family 551. OPISTHOMYZONID $A$}

Opisthomyzon Cope, 1889, 445.

\section{Family 552. ECHENEID $A$}

(Remoras; Shark-pilots)

Echeneis Linnæus, 1758, 12.

Remora Forster, 1771, 28.

Remora Catesby, 1771, 31.

Phtheirichthys Gill, 1862, 316.

Remilegia Gill, 1862, 316.

Remora Gill, 1862, 316.
Rhombochirus Gill, 1863, 324.

Remoropsis Gill, 1863, 324.

Leptecheneis Gill, 1864, 330.

Remorina Jordan \& Evermann, 1896, 475 . 


\section{Order JUGULARES (Deripia)}

\section{Series TRACHINIFORMES}

Family 553. TRACHINID瓜

This group forms a natural sequence from Trichodontida, Champsodontide, etc.

Trachinus Linnæus, 1758, 12.

Corystion Rafinesque, 1810, 79.

Pseudotrachinus Bleeker, 1862, 309.
Echiichthys Bleeker, 1862, 309. Pseudeleginus Sauvage, $1873,371$. Trachinopsis Sauvage, 1875, 380.

\section{Family 554. CALLIPTERYGID瓜}

Calltpteryx Agassiz, 1838, 191.

\section{Series NOTOTHENIIFORMES}

\section{Family. 555. NOTOTHENIID $Æ$}

Eleginus Cuvier \& Valenciennes, 1830 , 135.

Aphritis Cuvier \& Valenciennes, 1831, 137.

Notothenia Richardson, 1844, 222.

Macronotothen Gill, 1861, 306.

Eleginops Gill, 1861, 306, 455.

Pseudaphritis Castelnau, 1872, 575.

Phricus Berg, 1895, 466.

Dissostichus Smitt, 1898, 483.

Racovitzia Dollo, 1900, 489.

Artedidraco Lönnberg, 1905, 515.

Dolloidraco Roule, 1913, 551.

Pogonophryne Regan, 1914, 554.

Histiodraco Regan, 1914, 555.

\section{Family 556. BATHYDRACONID $\mathbb{E}$}

Bathydraco Günther, 1878, 394.

Gerlachea Dollo, 1900, 489.

Prinodraco Regan, 1914, 554.

Cygnodraco Waite, 1916, 561.

Aconichthys Waite, 1916, 561.

\section{Family 557. CHANNICHTHYID瓜}

Channichthys Richardson, 1844, 222.

Pagetodes Richardson, 1844, 222.

Chænichthys Richardson, 1844, 222.

Champsocephalus Gill, 1861, 306.

Ponerodon Alcock, 1890, 449.
Cryodraco Dollo, 1900, 489.

Chænocephalus Regan, 1913, 550.

Pagetopsis Regan, 1913, 550.

Chænodraco Regan, 1914, 554.

Dacodraco Waite, 1916, 561.

\section{Family 558. BOVICTID瓜}

Bovictus $^{470}$ Cuvier \& Valenciennes, 1831, Cottoperca Steindachner, 1875, 381. 137. Aurion Waite, 1916, 561.

${ }^{470}$ Later corrected to Bovichthys.

Family 559. HARPAGIFERID $\approx$

Harpagifer Richardson, 1844, 222 Sclerocottus Fischer (J. G.), 1885, 431. 


\section{Series CALLIONYMIFORMES}

\section{Family 560. DRACONETTID $\mathbb{E}$}

Draconetta Jordan \& Fowler, 1903, 505. Centrodraco Regan, 1913, 550.

\section{Family 561-562. CALLIONYMID五}

(Dragonets)

Callionymus Linnæus, 1758, 12.

Synchiropus Gill, 1859, 290.

Dactylopus Gill, 1859, 290.

Vulsus Günther, 1861, 307.
Calliurichthys Jordan \& Fowler, 1903, 505.

Draculo Snyder, 1911, 541.

Calymmichthys Jordan \& Thompson, 1914, 553.

\section{Series PERCOPHIDIFORMES \\ Family 563. PERCOPHIDID $\approx$}

Percophis Quoy \& Gaimard, 1824, 118.

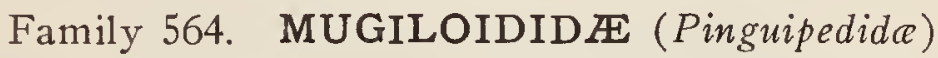

Mugiloides Lacépède, 1803, 67.

Myxonum Rafinesque, 1815, 90.

\section{Family 565. PARAPERCID $\approx$}

Percis Bloch \& Schneider, 1801, 58.

Parapercis Bleeker, 1863, 322.

Parapercis Steindachner, 1884, 430.

Neopercis Steindachner, 1885, 434.

Bathypercis Alcock, 1893, 460.

\section{Family 566. PTEROPSARID丑 (Bembropide)}

Bembrops Steindachner, 1876, 387.

Hypsicometes Goode, 1880, 403.

Bathypercis Alcock, 1880, 403.

Chrionema Gilbert, 1905, 513.
Macrias Gill \& Townsend, 1901, 493.

Pleuragramma Boulenger, 1902, 496.

Osurus Jordan \& Evermann, 1903, 506.

Chilias Ogilby, 1910, 536.

${ }^{480}$ A synonym of Acanthaphritis according to Regan, but the two genera differ in several respects.

\section{Family 567. HEMEROCETID丑 ,}

Hemerocœtes Cuvier \& Valenciennes,

$1837,189$.

\section{Family 568. CHIMARRHICHTHYID蚱}

Chimarrhichthys Haast, 1874, 375.

\section{Family 569. CREEDIID $\approx$}

Creedia Ogilby, 1898, 483.

\section{Family 570. LIMNICHTHYID压}

Limnichthys Waite, 1904, 511.

Schizochirus Waite, 1904, 511. 


\section{Family 571. TRICHONOTID E}

Trichonotus Bloch \& Schneider, 1801, 58. Lesueurina Fowler, 1907, 523.

Tæniolabrus Steindachner, 1867, 348.

Squamicreedia ${ }^{481}$ Rendahl, 1921.

Kræmeria Steindachner, 1906, 521.

"81 "Results of Dr. E. Mjöberg's Swedish Scientific Expeditions to Australia,191013," XVIII; Hjalmar Rendahl, "Fische," 20, 1921; orthotype Squamicredia obtusa Rendahl.

\section{Family 572. OXUDERCIDÆ}

Oxuderces Valenciennes, 1842, 213.

\section{Series AMMODYTIFORMES \\ Family 573. AMMODYTID $\mathbb{E}$}

(Sand-lances)

Ammodytes Linnæus, 1758, 12.

Hyperoplus Günther, 1862, 318.

Argyrotænia Gill, 1861.

\section{Family 574. BLEEKERIIDÆ}

Bleekeria Günther, 1862, 318.

Embolichthys Jordan, 1903, 504.

Rhynchias Gill, 1898, 482.

\section{Family 575. HYPOPTYCHID $Æ$}

Hypoptychus Steindachner, 1880, 406.

\section{Series BATHYMASTERIFORMES \\ Family 576. BATHYMASTERIDE}

(Ronquils)

Bathymaster Cope, 1873, 368.

Rathbunella Jordan \& Evermann, 1896,

Ronquilus Jordan \& Starks, 1895, 469. 474.

\section{Family 577. ZAPRORID王}

A group of uncertain relationships, having no ventral fins; the skeleton not studied. It may belong near Bathymaster, but it is perhaps an ally of Centrolophus, possibly of Cryptacanthodes.

Zaprora Jordan, 1896, 472.

Arkosteus Jordan \& Gilbert, 1920, 571.

\section{Series URANOSCOPIFORMES}

Family 578. CHIASMODONTID五

(Black Swallowers)

Chiasmodon ${ }^{482}$ Johnson, 1863, 327.

Pseudoseopelus Lütken, 1892, 458.
Dysalotus McGilchrist, 1905, 515.

Odontonema Weber, 1913, 551.

${ }^{42}$ Spelled Chiasmodus by Günther.

\section{Family 579. OPISTHOGNATHID $巴$}

(Jaw Fishes)

Opisthognathus (Cuvier) Oken, 1817, 101.

Gnathypops Gill, 1862, 316.
Lonchopisthus Gill, 1862, 316.

Stalix Jordan \& Snyder, 1902, 499.

Merogymnus Ogilby, 1908, 529. 


\section{Family 580. OWSTONIIDE}

Owstonia Tanaka, 1908, 531.

\section{Family 581. CHAMPSODONTID A}

Champsodon Günther, 1867, 345.

Centropercis Ogilby, 1895, 469.

\section{Family 582. URANOSCOPID $\mathbb{E}$}

\section{(Star-gazers)}

Uranoscopus Linnæus, 1758, 12.

Ichthyscopus Swainson, 1839, 202.

Astroscopus Brevoort, 1860, 295.

Kathetostoma ${ }^{483}$ Günther, 1860, 296.

Agnus Günther, 1860, 296.

Upsilonphorus Gill, 1861, 305.

Nematagnus Gill, 1861, 305.
Genyagnus Gill, 1861, 305.

Gnathagnus Gill, 1861, 305.

Synnema Haast, 1873, 369.

Ariscopus Jordan \& Snyder, 1902, 499.

Execestides Jordan \& Thompson, 1905, 514.

${ }^{4}$ Later written Cathetostoma.

\section{Family 583. LEPTOSCOPID $\approx$}

Leptoscopus Gill, 1859, 290.

Crapatalus Günther, 1861, 308.

\section{Family 584. DACTYLOSCOPID 2 E}

Dactyloscopus Gill, 1859, 290.

Crapatalus Günther, 1861, 208.

Myxodagnus Gill, 1861, 305.

Dactylagnus Gill, 1862, 318.
Gillellus Gilbert', 1890, 451.

Esloscopus Jordan \& Evermann, 1896, 474.

\section{- Series BLENNIIFORMES}

\section{Family 585. CLINID王}

Clinus Cuvier, 1817, 101.

Tripterygion $^{44}$ Risso, 1826, 119.

Myxodes Cuvier, 1829, 129.

Cirrhibarbus Cuvier, 1829, 129.

Enneapterygius Rüppell, 1835, 184.

Cristiceps Cuvier \& Valenciennes, 1836, 185.

Pterygocephalus Agassiz, 1839, 194.

Clinitrachus Swainson, 1839, 197.

Blennophis Swainson, 1839, 202.

Labrisomus Swainson, 1839, 202.

Ophisomus Swainson, 1839, 202.

Lepisoma Dekay, 1842, 210.

Heterostichus Girard, 1854, 258.

Neoclinus ${ }^{485}$ Girard, 1858.

Pterognathus Girard, 1859, 290.
Entomacrodus Gill, 1859, 291.

Malacoctenus Gill, 1860, 295.

Gobioclinus Gill, 1860, 295.

Blennioclinus Gill, 1860, 295.

Auchenionchus Gill, 1860, 296.

Calliclinus Gill, 1860, 296.

Ophthalmolophus Gill, 1860, 296.

Auchenopterus Günther, 1861, 307.

Cremnobates Günther, 1861, 308.

Gibbonsia Cooper, 1863, 323.

Lepidoblennius Steindachner, 1867, 348.

Lepidoblennius Sauvage, 1874, 376.

Blakea Steindachner, 1876, 387.

Acanthoclinus Mocquard, 1885, 433.

Petraites Ogilby, 1885, 434.

Paraclinus Mocquard, 1888, 443.

${ }^{48}$ Also corrected to Tripterygium.

${ }^{485}$ Girard, U.S.Pac. R. R. Surv., 10:114; orthotype Neoclinus blanchardi Girard.

(Omitted in "Genera of Fishes.") 
Dialommus Gilbert, 1890, 451.

Cryptotrema Gilbert, 1890, 451.

Cologrammus Gill, 1893, 461.

Notoclinus Gill, 1893, 461.

Ericentrus Gill, 1893, 461.

Scleroptery $x$ De Vis, 1894, 465.

Enneanectes Jordan \& Gilbert, 1895, 468.

Exerpes Jordan \& Evermann, 1896, 472.

Mnierpes J. \& E., 1896, 474.

Starksia Jordan \& Evermann, 1896, 472.

Emmnion Jordan, 1897, 477.

Pterognathus (Girard) Jordan \& Evermann, 1898, 481.

Corallicola Jordan \& Evermann, 1898, 481

Gillias Evermann \& Marsh, 1899, 485.

${ }^{488}$ Trop. Atl. Visch., 157; orthotype Histioclinus veliger Metzelaar.

\section{Family 586. NOTOGRAPTID $\approx$}

Notograptus Günther, 1867,345 .

Blanchardia Castelnau, 1875, 378.
Auchenistius Evermann \& Marsh, 1899, 485.

Coralliozetus E. \& M., 1899, 485.

Zacalles Jordan \& Snyder, 1902, 499.

Ericteis Jordan, 1904, 508.

Acteis Jordan, 1904, 508.

Sauvagea Jordan \& Seale, 1906, 519.

Congrammus Fowler, 1906, 517.

Calliblennius Barbour, 1912, 541.

Helcogramma McCulloch \& Waite, 1918, 564.

Trianectes M. \& W., 1918, 564.

Histioclinus ${ }^{488}$ Metzelaar, 1921.

Tekla Nichols, 1922. (See Index.)

Sticharium Günther, 1867, 345.

\section{Family 587. PERONEDYIDE}

Body eel-shaped, without pectoral fins, scales rudimentary; three lateral lines; dorsal mostly of spines.

Peronedys Steindachner, 1884, 430. Eucentronotus Ogilby, 1898, 483.

\section{Family 588. OPHIOCLINID王}

A group of slender, eel-shaped blennies, with rudimentary scales, the fin rays mainly spinous, here provisionally associated, their actual relationship undetermined.

Ophioclinus Castelnau, 1872, 363.

Neogunellus Castelnau, 1875, 378.

Neoblennius Castelnau, 1875, 378.

Stenophus Castelnau, 1875, 378.

\section{Family 589. BLENNIID E}

Blennius Linnæus, 1758, 12.

Salaria Forskål, 1775, 33.

Blennus Klein, 1779, 44.

Alticus (Commerson) Lacépède, 1800, 69.

Salarias Cuvier, 1817, 101.

Pholis Cuvier, 1817, 101.

Petroscirtes Rüppell, 1828, 173.

Aspidontus Quoy \& Gaimard, 1834, 180.

Ichthyocoris Bonaparte, 1836, 142, 206.

Chasmodes Cuvier \& Valenciennes, 1836, 185.

Alticus $^{487}$ (Commerson) Cuv. \& Va1., 1836.

Blennechis Cuv. \& Val., 1836, 185.

Omobranchus (Ehrenberg) Cuv. \& Val., 1836, 185.

Blennitrachus Swainson, $1839,198$.

Rupiscartes Swainson, 1839, 202.

Erpichthys Swainson, 1839, 202.

Cirripectes Swainson, 1839, 202.

Blennophis ${ }^{488}$ Valenciennes, 1844.

Adonis Gronow, 1854, 258.

Entomacrodus Gill, 1859, 289.

Ophioblennius Gill, 1860, 296.

Hypleurochilus Gill, 1861, 302.

${ }^{487}$ "Histoire Naturelle Poissons," XI, 337; the name mentioned but not accepted.

${ }^{488}$ Orthotype Blennophis webbi Valenciennes; preoccupied; replaced by Ophioblennius Gill. 
Hypsoblennius Gill, 1861, 302.

Salarichthys ${ }^{489}$ Guichenot, $1867,346$.

Enchelyurus Peters, 1868, 353.

Alticus (Commerson) Bleeker, 1869, 353.

Isesthes Jordan \& Gilbert, 1882, 420.

Scartella Jordan, 1886, 435.

Lipophrys Gill, 1896, 471.

Scartes Jordan \& Evermann, 1896. 475.

Macrurrhynchus Ogilby, 1896, 475.

Homesthes Gilbert, 1898, 481.
Blenniolus Jordan \& Evermann, 1898, 481.

Scartichthys Jordan \& Evermann, 1898, 481.

OnColepis Bassani, 1898, 478.

Brannerella Gilbert, 1900, 489.

Graviceps Fowler, 1903, 502.

Exallias Jordan \& Evermann, 1905, 514.

Cyneichthys Ogilby, 1910, 536.

Parablennius Ribeiro, 1915, 557.

${ }^{400}$ Written also Salariichthys.

\section{Family 590. EMBLEMARIID $2 \mathrm{E}$}

This family and the three which follow are entirely provisional, designed to receive aberrant genera which by differences in dentition confuse the definition of Blenniida.

Emblemaria Jordan \& Gilbert, 1882, $420 . \quad$ Acanthemblemaria ${ }^{400}$ Metzelaar, 1920.

400 "Trop. Atl. Visschen," 159; orthotype A. spinosa Metzelaar.

\section{Family 591. RUNULIDÆ}

A group of naked blennies with the mouth small, transverse, and inferior, the members perhaps not related one to another.

Andamia Blyth, 1859, 291.

Heteroclinus Castelnau, 1872, 363.
Runula Jordan \& Bollman, 1889, 447.

Runulops Ogilby, 1910, 536.

\section{Family 592. ATOPOCLINID无}

Teeth solid, trenchant.

Atopoclinus Vaillant, 1894, 466.

\section{Family 593. CH ENOPSID无}

A provisional group of elongate, naked blennies of the tropics, perhaps unrelated among themselves, and resembling on one hand the Pholide and on the other the Clinida.

Pholidichthys Bleeker, 1856, 267.

Gunnellichthys Bleeker, 1858, 277.

Chænopsis Gill, 1863, 323.

Stathmonotus Bean, 1885, 430.
Psednoblennius Jenkins \& Evermann, 1888, 442.

Lucioblennius Gilbert, 1890, 451.

\section{Family 594. CEBIDICHTHYID无}

The three genera here associated differ from the allied Pholida in having the posterior half of the dorsal fin of soft rays.

Cebidichthys $^{101}$ Ayres, 1855, 262.

Zoarchias Jordan \& Snyder, 1902, 499.

Neozoarces Steindachner, 1880, 406.

${ }^{401}$ Often misspelled Cebedichthys. 


\section{Family 595. PHOLID $Æ$}

A varied group of subarctic blennies which may require further subdivision.

Pholis Gronow, 1763, 19.

Pholis (Gronow) Scopoli, 1777, 42.

Pholis Röse, 1793, 52.

Pholidus Rafinesque, 1815, 88.

Murænoides Lacépède, 1800, 56.

Centronotus Bloch \& Schneider, 1801, 58.

Dactyleptus Rafinesque, 1815, 88.

Gunnellus Cuvier \& Valenciennes, 1836, 185.

Ophisomus Swainson, 1839, 202.

Chirolophis Swainson, 1839, 202.

Carelophus Kröyer, 1845,"226.

Dictyosoma Temminck \& Schlegel, 1845, 227.

Apodichthys Girard, 1854, 258.

Blenniops Nilsson, 1855, 266.

Anoplarchus Gill, 1861, 305.

Asternopteryx (Rüppell) Günther, 1861, 307.

Urocentrus Kner, 1868, 352.

Opisthocentrus Kner, 1868, 352.
Gunnellops Bleeker, 1874, 372.

Plectobranchus Gilbert, 1890, 451.

Blenniophidium Boulenger, 1892, 457.

Plagiogrammus Bean, 1893, 460.

Xererpes Jordan \& Gilbert, 1895, 469.

Bryostemma Jordan \& Starks, 1895, 469.

Rhodymenichthys Jordan \& Evermann, $1896,475$.

Ulvicola Gilbert \& Starks, 1897, 477.

Alectrias Jordan \& Evermann, 1898, 482.

Pholidapus Bean \& Bean, 1896, 471.

Enedrias Jordan \& Evermann, 1898, 481.

Eulophias Smith, 1902, 500.

Azuma Jordan \& Snyder, 1902, 499.

Bryolophus J. \& S., 1902, 499.

Abryois J. \& S., 1902, 499.

Trigrammus Gracianov, 1907, 524.

Askoldia Pavlenko, 1910, 537.

Gymnoclinus Gilbert \& Burke, 1912, 543.

Alectridium Gilbert \& Burke, 1912, 543.

\section{Family 596. XIPHISTERID五 (Xiphidiontida)}

Xiphidion $^{422}$ Girard, 1857, 290.

Xiphister Jordan, $1879,400$.

Xiphistes Jordan \& Starks, 1895, 469.

Phytichthys ${ }^{433}$ Hubbs, 1923.

${ }^{492}$ Preoccupied by Xiphidion Seville, a genus of Orthoptera, the word later corrected to Xiphidium. Xiphidion was first used by Girard, U. S. Pac. R. R. Surv., 6: 20, 1857.

${ }^{483}$ Hubbs, ms. : new genus; orthotype Xiphister chirus Jordan \& Gilbert; substitute for Xiphistes, preoccupied in insects.

\section{Family 597. STICHÆID压}

Stichæus ${ }^{24}$ Reinhardt, 1837.

Eume sogrammus Gill, 1864, 331.

Stichæopsis Steindachner \& Kner, 1870, 360.

Notogrammus ${ }^{108}$ Bean, 1881.
Dinogunnellus Herzenstein, 1890, 451.

U1varius Jordan \& Evermann, 1896, 475.

Ernogrammus J. \& E., 1898, 481.

Ozorthe J.\& E., 1898, 481.

Trigrammus Gracianov, 1907, 524.

Dansk. Vid. Nat. Afh., 109; orthotype Sticha punctatus Reinhardt. (Omitted in "Genera of Fishes.")

${ }^{498}$ Bean, Proc. U.S. Nat. Mus., 4:147; orthotype N, rothrocki Bean. A synonym of Stichaus. (Omitted in "Genera of Fishes.") 
Family 598. LUMPENID压

Lumpenus ${ }^{408}$ Reinhardt, 1837.

Ctenodon Nilsson, 1855, 266.

Leptogunnellus Ayres, 1855, 262.

Leptoblennius Gill, 1860, 295.
Leptoclinus Gill, 1861, 302.

Centroblennius Gill, 1861, 302.

Anisarchus Gill, 1864, 331.

Poroclinus Bean, 1890, 450.

${ }^{190}$ Dansk. Vid. Selsk. Nat., 6: 110; orthotype Blennius lumpenus Fabricius, not of Linnæus. (Omitted in "Genera of Fishes.")

Family 599. PTILICHTHYID压

Ptilich thys Bean, 1881, 415.

\section{Family 600. CRYPTACANTHODID $~ \approx$}

Cryptacanthodes Storer, 1839, 197.

Delolepis Bean, 1881, 415.

Lyconectes Gilbert, 1895, 467.

Family 601. ANARHICHADID $\mathbb{E}$

Anarchichas ${ }^{407}$ Linnæus, $1758,12$.

Lathargus $^{488}$ Klein, 1775, 38.

Palanarrhichas LeHon, 1871, 361.

${ }^{407}$ Of ten spelled Anarrhichas.

${ }^{698}$ Misprinted Latargus.

\section{Family 602. ANARRHICHTHYID丑}

Anarrhichthys Ayres, 1855, 262.

Lycichthys Gill, 1877, 389.

LAPARUS (Agassiz) Woodward, (1845) 1901, 224.

\section{Family 603. XIPHASIID $\approx$}

Xiphasia $^{498}$ Swainson, 1839, 201.

Xiphogadus Günther, 1862, 318.

Nemophis Kaup, 1858, 281.

Plagiotremus Gill, 1863, 323.

${ }^{400}$ Also spelled Ziphasia by Swainson.

Family 604. XENOCEPHALID $\mathbb{E}$

Xenocephalus Kaup, 1858, 281.

\section{Series ZOARCIFORMES}

\section{Family 605. CONGROGADID $\mathbb{E}$}

Haliophis Rüppell, 1828, 122.

Machærium Richardson, 1843, 216.

Congrogadus Günther, 1862, 318.
Halidesmus Günther, 1871, 361.

Blennodesmus Günther, 1871, 361.

Hierichthys Jordan \& Fowler, 1902, 499.

Family 606. CERDALID王 (Microdesmida)

Microdesmus Günther, 1864, 333.

Cerdale Jordan \& Gilbert, 1881, 416.

Leptocerdale Weymouth, 1910, 537.

\section{Family 607. SCYTALINID $\mathrm{E}$}

Scytalina Jordan \& Gilbert, 1880, 404. Scytaliscus Jordan \& Gilbert, 1883, 424. 


\section{Family 608. ZOARCIDE (Lycodida)}

Enchelyopus Gronow, 1763, 19.

Zoarces $^{600}$ Cuvier, 1829, 129.

Lycodes Reinhardt, 1838, 193.

Gymnelis Reinhardt, 1838, 193.

Phycocœtes Jenyns, 1842, 212.

Iluocœtes Jenyns, 1842, 212.

Uronectes Günther, 1862, 318.

Macrozoarces Gill, 1863, 325.

Maynea Cunningham, 1871, 361.

Paralycodes Bleeker, 1874, 372.

Lycodalepis Bleeker, 1874, 372.

Lycodopsis Collett, 1879, 399.

Leurynnis Lockington, 1879, 400.

Hypolycodes Hector, 1880, 403.

Melanostigma Günther, 1881, 416.

Bassozetus Gill, 1883, 424.

Lycocara Gill, 1884, 428.

Lycenchelys Gill, 1884, 428.

Gymnelichthys Fischer, 1885, 431.

Lycodophis Vaillant, .1888, 444.

Lyconema Gilbert, 1895, 467.
Nemalycodes Herzenstein, 1896, 472.

Furcella Jordan \& Evermann, 1896, 475.

Aprodon Gilbert, 1890, 451.

Platea Steindachner, 1898, 484.

Lycias Jordan \& Evermann, 1898, 481.

Embryx J. \& E., 1898, 481.

Furcimanus J. \& E., 1898, 482.

Selachophidium Gilchrist, 1903, 503.

Petrotyx Heller \& Snodgrass, 1903, 503.

Eutyx Heller \& Snodgrass, 1903, 503.

Krusensterniella Schmidt, 1904, 511.

Hadropareia Schmidt, 1904, 511.

Pachycara Zugmayer, 1911, 541.

Mastigopterus Radcliffe, 1913, 549.

Ophthalmolycus Regan, 1913, 550.

Austrolycus Regan, 1913, 550.

Austrolycichthys Regan, 1913, 550.

Crossolycus Regan, 1913, 550.

Zoarcites Zugmeyer, 1914, 555.

Lycogramma Gilbert, 1915, 556.

${ }^{500}$ Also spelled Zoarchus.

Family 609. LYCODAPODID压

Lycodapus Gilbert, 1890, 451.

Snyderidia Gilbert, 1905, 513.

\section{Family 610. DEREPODICHTHYID尼}

Derepodichthys Gilbert, 1895, 467.

\section{Series BROTULIFORMES}

\section{Family 611. BROTULID E}

Brotula $^{501}$ Cuvier, 1829.

Bythites Reinhardt, 1837, 190.

Tilurus ${ }^{\mathrm{s02}}$ Kölliker, 1854, 254.

Dinematichthys Bleeker, 1855, 263.

Gadopsis Filippi, 1855, 265.

Brotulophis Kaup, 1858, 281.

Brotella Kaup, 1858, 281.

Hoplophycis Kaup, 1858, 281.

Sirembo Bleeker, 1858, 277.

Halias Ayres, 1859, 293.

Lucifuga Poey, 1860, 298.

Brosmophycis Gill, 1861, 305.
Nematobrotula Gill, 1863, 325.

Stygicola Gill, 1863, 325.

Hoplobrotula Gill, 1863, 325.

Acanthonus Günther, 1878, 394.

Bathyriectes Günther, 1878, 394.

Aphyonus Günther, 1878, 394.

Typhlonus Günther, 1878, 394.

Dicrolene Goode \& Bean, 1882, 419.

Lycodonus Goode \& Bean, 1882, 419.

Poromitra Goode \& Bean, 1882, 419.

Barathrodemus Goode \& Bean, 1882, 419.

${ }^{501}$ Régne Animal; type Enchelyopus barbatus Bloch and Schneider. (Omitted in "Genera of Fishes.")

${ }^{502}$ This genus and its allies, Tilurella, Tiluropis, and Grimaldichthys, are larval forms, supposed to be of brotulids. 
Bellottia Giglioli, 1883, 423.

Porogadus Goode \& Bean, 1885, 432.

Bathyonus Goode \& Bean, 1885, 432.

Neobythites Goode \& Bean, 1885, 432.

Barathronus Goode \& Bean, 1886, 435.

Myxocephalus Steindachner \& Döderlein, 1887, 439.

Catatyx Günther, 1887, 437.

Pteroidonus Günther, 1887, 437.

Mixonus Günther, 1887, 437.

Diplacanthopoma Günther, 1887, 437.

Nematonus Günther, 1887, 437.

Alexeterion Vaillant, 1888, 444.

Pycnocraspedum Alcock, 1889, 444.

Paradicrolene Alcock, 1889, 444.

Saccogaster Alcock, 1889, 444.

Glyptophidium Alcock, 1889, 444.

Dermatorus Alcock, 1890, 449.

Monomitopus Alcock, 1890, 449.

Paradicrolene Alcock, 1890, 449.

Bothrocara Bean, 1890, 450.

Lamprogrammus Alcock, 1891, 454.

Hephthocara Alcock, 1892, 457.

Benthocometes Goode \& Bean, 1895, 468.

Dicromita G. \& B., 1895, 468.

Celema G. \& B., 1895, 468.

Penopus G. \& B., 1895, 468.

Mœbia G. \& B., 1895, 468.

Alcockia r. \& B., 1895, 468.

Dermatopsis Ogilby, 1896, 475.
Monothrix Ogilby, 1897, 477.

Ogilbia Jordan \& Evermann, 1898, 480.

Diancistrus Ogilby, 1898, 483.

Holcomycteronus Garman, 1899, 486.

Pseudonus Garman, 1899, 486.

Leucicorus Garman, 1899, 486.

Sciadonus Garman, 1899, 486.

Eretmichthys Garman, 1899, 486.

Bothrocaropsis Garman, 1899, 486.

Watasea Jordan \& Snyder, 1901, 494.

Dipulus Waite, 1905, 516.

Bassobythites Brauer, 1906, 517.

Barathrites Zumayer, 1911, 541.

Leucochlamys ${ }^{503}$ Zugmayer, 1911.

Parabrotula ${ }^{504}$ Zugmayer, 1911.

Tilurella Roule, 1911, 541.

Tiluropsis Roule, 1911, 541.

Xenobythites Radcliffe, 1913, 549.

Pyramodon Radcliffe, 1913, 549.

Hypopleuron Radcliffe, 1913, 549.

Mastigopterus Radcliffe, 1913, 549.

Homostolus Radcliffe, 1913, 549.

Enchelybrotula Radcliffe, 1913, 549.

Hypopleuron Radcliffe, 1913, 549.

Luciobrotula Radcliffe, 1913, 549.

Grimaldichthys Roule, 1913, 551.

Pyramodon Regan, 1914, 555.

Spectrunculus Jordan \& Thompson, 1914, 553.

Cynophidium Regan, 1914, 554.

${ }^{{ }^{00}}$ Rés. Camp. Sci. Monaco, 131; type Leucochlamys cryptophthalmus Zugmayer. (Omitted in "Genera of Fishes.")

${ }^{\circ 04}$ Loc. cit., 129; orthotype Parabrotula plagiosphelmus Zugmayer. (Omitted in "Genera of Fishes.")

\section{Series OPHIDIIFORMES}

\section{Family 612. RHODICHTHYIDA}

Rhodichthys Collett, 1878, 393.

\section{Family 613. OPHIDIID $Æ$}

Ophidion $^{805}$ Linnæus, 1758, 12.

Xiphiurus Smith, 1849, 244.

Cepolophis Kaup, 1856, 272.

Genypterus Philippi, 1857, 276.

Leptophidium Gill, 1863, 324.
Otophidium Gill, 1885, 433.

Lepophidium Gill, 1895, 467.

Chilara Jordan \& Evermann, 1896, 475.

Rissola J. \& E., 1896, 475.

Pseudophidium Gracianov, 1907, 524.

${ }^{\text {sos }}$ Often spelled Ophidium. 


\title{
Series CARAPIFORMES
}

\section{Family 614. CARAPIDE (Fierasferid $e$ )}

\author{
(Pearl Fishes)
}

Carapus $^{508}$ Rafinesque, 1810.

Fierasfer (Cuvier) Oken, 1817, 93, 101.

Echiodon Thompson, 1837, 190.

Encheliophis Müller, 1843, 215.

Diaphasia Lowe, 1843, 215.

Oxybeles Richardson, 1844, 223.

Porobronchus Kaup, 1860, 297.
Helminthodes Gill, 1864, 331.

Helminthostoma Cocco, 1870, 357.

Vexillifer Gasco, 1870, 357.

Lefroyia Jones, 1874, 375.

Rhizoiketicus Vaillant, 1893, 463.

Jordanicus Gilbert, 1905, 513.

${ }^{800}$ A genus based on a definition borrowed from Lacépède and including two unrelated forms; one Fierasfer; one Gymnotus; no type named. By a decision of the International Commission of Zoological Nomenclature, Carapus is held to replace Fierasfer, as the species first referred to it by a subsequent author was Ohpidium imberbe Linnæus.

\section{Suborder HAPLODOCI}

\section{Family 615. BATRACHOIDIDE}

Batrachoides Lacépède, 1800, 57.

Batrachus Bloch \& Schneider, 1801, 57.

Batrictius Rafinesque, 1815, 88.

Opsanus Rafinesque, 1818, 108.

Amphichthys Swainson, 1839, 202.

Porichthys Girard, 1854, 258.

Thalassophryne Günther, 1861, 307.

Halophryne Gill, 1863, 324.

Pseudobatrachus Castelnau, 1875, 378.
Phrynotitan Gill, 1885, 432.

Marcgravia Jordan, 1886, 435.

Thalassothia Berg, 1895, 466.

Dæctor Jordan \& Evermann, 1898, 481.

Coryzichthys Ogilby, 1908, 529.

Halobatrachus Ogilby, 1908, 529.

Batrachomcus Ogilby, 1908, 529.

Marcgravichthys Ribeiro, 1915, 557.

Nautopædium Jordan, 1919, 567.

\section{Order XENOPTERYGII (Xenopteri)}

Family 616. GOBIESOCID死

Lepadogaster Gouan, 1770, 28.

Gobiesox Lacépède, $1800,57$.

Piescephalus Rafinesque, $1810,81$.

Megaphalus Rafinesque, 1815, 89.

Gouania Nardo, 1832, 176.

Rupisuga Swainson, 1839, 206.

Cotylis Müller, 1843, 215.

Sicyases Müller, 1843, 216.

Trachelochismus de Barneville, 1846, 228.

Chorisochismus de Barneville, 1846, 228.

Sicyogaster Brisout de Barneville, 1846, 228.

Tomicodon Brisout de Barneville, 1846, 228.

Apepton Gistel, 1848, 236.
Leptopterygius Troschel, 1860, 300.

Crepidogaster Günther, 1861, 307.

Diplocrepis Günther, 1861, 307.

Caularchus Gill, 1862, 317.

Mirbelia Canestrini, 1864, 329.

Rimicola Jordan \& Evermann, 1896, 472.

Arbaciosa J. \& E., 1896, 472.

Bryssetæres J. \& E., 1896, 472.

Caulistius J. \& E., 1896, 475.

Bryssophilus J. \& E., 1898, 481.

Aspasma Jordan \& Fowler, 1902, 498.

Lepadichthys Waite, 1904, 511.

Aspasmagaster Waite, 1907, 526.

Bulbiceps Jordan, 1919, 568.

Cotylichthys Jordan, 1919, 567. 


\section{Order PLECTOGNATHI}

\section{Suborder SCLERODERMI}

This group is closely related to the Squamipinnes, especially to the Acanthurida, from ancestors of which it must have been derived.

\section{Family 617. SPINACANTHID无}

Spinacanthus Agassiz, 1844, 219.

Protobalistum (Massalongo) Zigno, 1887, 439.

\section{Family 618. TRIACANTHID $\mathbb{E}$}

Triacanthus (Cuvier) Oken, 1817, 98. Acanthopleurus Agassiz, 1844, 217.

Triacanthodes Bleeker, 1858, 277.

\section{Family 619.}

Balistes Linnæus, 1758, 15.

Capriscus Klein, 1777, 43.

Capriscus Röse, 1793, 52.

Capriscus Rafinesque, $1810,82$.

Balistapus Tilesius, 1820, 113.

Xenodon Rüppell, 1835, 184.

Pachynathus ${ }^{507}$ Swainson, 1839, 199.

Zenodon ${ }^{\text {s08 }}$ Swainson, 1839, 204.

Rhinecanthus Swainson, 1839, 204.

Chalisoma Swainson, 1839, 204.

Canthidermis Swainson, 1839, 204.

Melichthys $^{509}$ Swainson, 1839, 204.

Capriscus Swainson, 1839, 204.

Leiurus Swainson, 1839, 205.
Hollardia Poey, 1861, 308.

Halimochirus Alcock, 1899, 484.

Tydemania Weber, 1913, 551.

\section{BALISTID正}

AnCistrodon $^{510}$ (Debey) Roemer, 1843.

Acanthoderma Agassiz, 1844, 217.

Agoreion Gistel, 1848, 237.

Odonus Gistel, 1848, 238.

Erythrodon Rüpell, 1852, 251.

Pyrodon Kaup, 1855, 265.

Xanthichthys Kaup, 1856, 274.

Pseudobalistes Bleeker, 1866, 340.

Parabalistes Bleeker, 1866, 340.

An KISTROdUs Koninck, 1870, 358.

Protacanthodes Gill, 1888, 441.

GRYPODON $^{511}$ Hay, 1899.

Abalistes Jordan \& Seale, 1906, 519.

Sufflamen Jordan, 1916, 559.

${ }^{507}$ Regarded by the International Commission as a misprint for Pachygnathus, a name already in use.

${ }^{508}$ Perhaps a misprint for Xenodon.

${ }^{000}$ Written later Melanichthys.

"10 "Texas," 420; orthotype Ancistrodon texanus Roemer. (Omitted in "Genera of Fishes.")

${ }^{\text {s11 }}$ Substitute for Ancistrodon; preoccupied in snakes.

\section{Family 620. MONACANTHID疋}

Unicornis Catesby, 1771, 31.

Monoceros (Plumier) Lacépède, 1800, 71.

Monacanthus (Cuvier) Oken, 1817, 98.

Alutera $^{\text {s12 }}$ (Cuvier) Oken, 1817, 98.

Amanses Gray, 1833, 139, 179.

Cantherines Swainson, 1839, 205.

Chætodermis Swainson, 1839, 205.
Trichoderma Swainson, 1839, 205.

Stephanolepis Gill, 1861, 304.

Ceratacanthus Gill, 1861, 303.

Pseudaluterius $^{\text {818 }}$ Bleeker, 1865, 336.

Pseudomonacanthus Bleeker, 1866, 340.

Acanthaluteres Bleeker, 1866, 340.

Brachaluteres Bleeker, 1866, 340.

${ }^{12}$ Variously written Aleuteres, Alutarius, Aleisterius, etc. 
Pseudaluteres ${ }^{518}$ Bleeker, 1866, 340.

Paraluteres Bleeker, 1866, 340.

Oxymonacanthus Bleeker, 1866, 340.

Paramonacan thus Bleeker, 1866, 340.

Liomonacanthus Bleeker, 1866, 340.
Paramonacanthus Steindachner, 1867, 348.

Osbeckia Jordan \& Evermann, 1896, 474.

Rudarius Jordan \& Fowler, 1902, 498.

Davidia Ribeiro, 1915, 557.

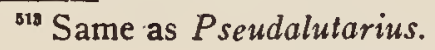

\section{Family 621. PSILOCEPHALID王}

Anacanthus Gray, 1833.

Pogonognathus Bleeker, 1849, 240.

Psilocephalus Swainson, 1839, 205.

\section{Suborder OSTRACODERMI}

\section{Family 622. OSTRACIIDA}

Ostracion Linnæus, 1758, 15.

Acarana Gray, 1833, 139, 179, 192.

Lactophrys Swainson, 1839, 204.

Rhinesomus Swainson, 1839, 204.

Tetrosomus Swainson, 1839, 204.

Platycanthus Swainson, 1839, 204.

Anoplocapros Kaup, 1855, 265.

Kentrocapros Kaup, 1855, 265.

Cibotion (Klein) Kaup, 1855, 265.

Capropygia Gray, 1855, 265.

Acanthostracion Bleeker, 1866, 339.

Chapinus Jordan \& Evermann, 1896, 474.

Lactoria Jordan \& Fowler, 1902, 498.

Caprichthys McCulloch \& Waite, 1915, 557.

\section{Suborder GYMNODONTES}

Family 623. TRIODONTID压

Triodon Cuvier, 1829, 131.

\section{Family 624. TETRAODONTID瓜 (Chonerhinida)}

Tetraodon $^{\text {s14 }}$ Linnæus, 1758, 15.

Orbis Catesby, 1771, 31.

Crayracion Klein, 1777, 12.

Ovoides $^{515}$ Lacépède, 1798.

Sphœroides ${ }^{515}$ Lacépède, 1798.

Ovoides Cuvier, 1800, 55.

Orbis (Plumier) Lacépède, 1800, 71.

Ovum Bloch \& Schneider, 1801, 60.

Spheroides (Lacépède) Duméril, 1806,

$55,75$.

Ovoides (Lacépède) Duméril, 1806, 75.

Orbis Fischer, 1813, 85.

Oonidus Rafinesque, 1815, 91.

Orbidus Rafinesque, 1815, 91.
Physogaster Müller, 1839, 196.

Arothron Müller, 1839, 196.

Cheilichthys Müller, 1839, 196.

Chelonodon Müller, 1839, 196.

Leiodon Swainson, 1839, 198.

Lagocephalus Swainson, 1839, 205.

Cirrhisomus Swainson, 1839, 205.

Leisomus Swainson, 1839, 205.

Gastrophysus Müller, 1843, 216.

Anchisomus Kaub, 1854, 261.

Holacanthus Gronow, 1854, 258.

Chonerhinos $^{\text {s10 }}$ Bleeker, 1854, 256.

Dichotomycter Bibron, 1855, 263.

Epipedorhynchus Bibron, 1855, 263.

"Often written Tetrodon.

${ }^{115}$ In the Allgemeine Literar Zeitung, Berlin, No. 287, 1798, is an unsigned review of Lacépède's Volumes I and II, Latin names are supplied page 674 to "les Ovoides," and page 675 to "les Spheroides." Sphoroides of this reviewer, 1798, replaces Spheroides Duméril, 1806. For this reference I am indebted to Mr. Remington Kellogg.

${ }^{\text {sio }}$ Usually corrected to Chonerhinus. 
Geneion Bibron, 1855, 263.

Catophorhynchus Bibron, 1855, 263.

Batrachops Bibron, 1855, 263.

Monotreta $^{817}$ Bibron, 1855, 263.

Ephippion Bibron, 1855, 263.

Aphanacanthus Bibron, 1855, 263.

Xenopterus Bibron, 1855, 263.

Amblyrhynchotus Bibron, 1855, 263.

Stenometopus Bibron, 1855, 263.

Dilobomycter Bibron, 1855, 263.

Promecocophalus Bibron, 1855, 262.
Apsicephalus Hollard, 1857, 275.

Brachycephalus Hollard, 1857, 275.

Pleuranacanthus (Bibron) Bleeker, 1865, 336.

Uranostoma (Bibron) Bleeker, 1865, 336.

Crayracion (Klein) Bleeker, 1865, 339.

Hemiconiatus Günther, 1870, 357.

Liosaccus Günther, 1870, 357.

Guentheridia Gilbert \& Starks, 1904, 508.

${ }^{* 11}$ Also spelled Monotretus.

\section{Family 625. CANTHIGASTERIDE (Tropidichthyide)}

(Sharp-nosed Puffers)

Canthigaster Swainson, 1839, 198.

Psilonotus Swainson, 1839, 205.

Prilonotus $^{518}$ (Kaup) Müller, 1854, 261.

Tropidichthys Bleeker, 1854, 256.
Rhynchotus Bibron, 1855, 263.

Anosmius Peters, 1855, 266.

Eumycterias Jenkins, 1901, 493.

${ }^{518}$ Perhaps a slip for Psilonotus.

\section{Family 626. DIODONTID正}

Diodon Linnæus, 1758, 15.

Orbis Müller (P. L. S.), 1767, 24.

Chilomycterus Bibron, 1846, 228.

Megalurites Costa, 1850, 245.

ENNEODON Heckel, 1854, 259.

Cyclichthys Kaup, 1855, 265.

Cyanichthys Kaup, 1855, 265.

Dicotylichthys Kaup, 1855, 265.

Heptadiodon Bronn, 1855, 264.

Atopomycterus $^{519}$ (Verraux) Bleeker,

Paradiodon Bleeker, 1865, 336.

Trichodiodon Bleeker, 1866, 340.

Trichocyclus Günther, 1870, 357.

Gymnodus Delfortrie, $1871,361$.

Progymnodon Dames, 1883, 422.

Lyosphæra Evermann \& Kendall, 1898, 480.

Euchilomycterus Waite, 1900, 491.

Allomycterus ${ }^{620}$ McCulloch, 1921. $1865,336$.

${ }^{2110}$ Orthotype $A$. diversispinis Verraux; incorrectly stated in "Genera of Fishes."

${ }^{200}$ Rec. Austr. Mus., 13: 141; orthotype Diodon jaculiferus Cuvier.

\section{Family 627. MOLIDE (Orthagoriscida)}

Mola Kœlreuter, 1770, 28.

Mola Linck, 1790, 49.

Mola Cuvier, $1798,54$.

Orthagoriscus Bloch \& Schneider, 1801, 60.

Cephalus Shaw, 1804, 73.

Orthragus Rafinesque, 1810, 78.

Diplanchias Rafinesque, 1810, 78.

Pedalion Swainson, 1838, 193.

Ozodura Ranzani, 1839, 196.
Tympanomium Ranzani, 1839, 196.

Trematopsis Ranzani, 1839, 196.

Pallasia Nardo, 1839, 196.

Ranzania Nardo, 1839, 196.

Molacanthus Swainson, 1839, 205.

Acanthosoma Dekay, 1842, 210.

Centaurus Kaup, 1855, 265.

Masturus Gill, 1884, 428.

Chelonopsis ${ }^{\text {s21 }}$ Jordan, 1900.

"Gr1 "Guide to the Study of Fishes," II, 425. This name, never intended as generic, is a printer's error for Mola chelonopsis, a fossil Mola. 


\section{Order PEDICULATI}

\section{Family 628. LOPHIID $Æ$}

Lophius Linnæus, 1758, 11.

Batrachus Klein, 1775, 40.

Lophidius Rafinesque, 1815, 92.

Lophiopsides Guichenot, 1867, 345.
Lophiomus Gill, 1882, 419.

Lophiodes Goode \& Bean, 1895, 468.

Chirolophius Regan, 1903, 505.

Sladenia Regan, 1908, 530.

\section{Family 629. ANTENNARIID $\approx$}

Antennarius (Commerson) Lacépède, 1798, 69.

Histrio ${ }^{522}$ Fischer, 1813, 84.

Antennarius (Commerson) Cuvier, 1817, 104.

Chironectes Cuvier, 1817, 96.

Batrachopus ${ }^{823}$ Goldfuss, 1820, 114, 172.

Capellaria Gistel, 1848, 235.

Pterophryne Gill, 1863, 324:
Saccarius Günther, 1861, 307.

Histiophryne Gill, 1863, 324.

Pterophrynoides ${ }^{544}$ Gill, 1878.

Tetrabrachium Günther, 1880, 403.

Histiocephalus Zigno, 1890, 453.

Histionotopterus Eastman, 1904, 507.

Trianectes McCulloch, 1918, 564.

Echinophryne McCulloch, 1918, 564.

Trichophryne McCulloch, 1918, 564.

${ }^{62}$ This name may be questioned on account of an error in the published diagnosis, which transposes the generic character of Lophius and Histrio.

${ }^{223}$ Misprinted Batrachops on page 114.

${ }^{324}$ Proc. U. S. Nat. Mus., 1:215; orthotype Lophius histrio Gill; a substitute for Pterophryne, regarded as preoccupied by Pterophrynus, a synonym of Histrio if the latter is eligible. (Omitted in "Genera of Fishes.")

\section{Family 630. BRACHIONICHTHYID $\approx$}

Brachionichthys Bleeker, 1855, 263. Sympterichthys Gill, 1878, 394.

\section{Family 631. CHAUNACID $\approx$}

Chaunax Lowe, 1846, 229.

Family 632. OGCOCEPHALIDE (Oncocephalida; Malthida)

Ogcocephalus ${ }^{525}$ Fischer, 1813, 85.

Malthe ${ }^{528}$ Cuvier, 1817, 105.

Halieutæa Cuvier \& Valenciennes, 1837, 189.

Astrocanthus Swainson, 1839, 205.

Halieutichthys Poey, 1863, 324.

Dibranchus Peters, 1875, 379.

Halieutella Goode \& Bean, 1885, 432.

Allector Heller \& Snodgrass, 1903, 503.

Cœlophrys Brauer, 1902, 497.

Brephostoma Alcock, 1889, 444.
Halieutopsis Garman, 1899, 486.

Dibranchichthys Garman, 1899, 486.

Dibranchopsis Garman, 1899, 486.

Halicmetus Alcock, 1891, 454.

Malthopsis Alcock, 1891, 454.

Zalieutes $^{627}$ Jordan \& Evermann, 1896.

Eschynichthys Ogilby, 1907, 526.

Tathicarpus Ogilby, 1907, 526.

Rhycherus Ogilby, 1907, 526.

Dermatias Radcliffe, 1912, 544.

${ }^{625}$ Sometimes corrected to Oncocephalus.

${ }^{626}$ Afterwards written Malthea.

${ }^{627}$ Jordan \& Evermann, "Check List," 511; type Malthe elater Jordan \& Gilbert. (Omitted in "Genera of Fishes.") 


\section{Family 633. CERATIID正}

Ceratias Kröyer, 1845, 227.

Mancalias Gill, 1878, 394.

Typhlopsaras Gill, 1883, 423.

Cryptopsaras Gi11, 1883, 423.

Miopsaras Gilbert, 1905, 513.
Paraceratias Tanaka, 1908, 531.

Neoceratias Pappenheim, 1914, 554.

Monoceratias Gilbert, 1915, 556.

Thaumatichthys Smith \& Radcliffe, $1912,546$.

\section{Family 634. HIMANTOLOPHIDE}

Himantolophus Reinhardt, 1838, $193 . \quad$ Diceratias Günther, 1887, 437.

Oneirodes ${ }^{528}$ Lütken, 1871, 362.

Corynolophus $^{529}$ Gill, 1878. Linophryne Collett, 1886, 434.

Egæonichthys Clarke, 1878, 394, 575.

${ }^{523}$ Misprinted Oneiroides in "Genera of Fishes."

${ }^{520}$ Proc. U. S. Nat. Mus., 219; type Himantolophus reinhardti Lütken. (Omitted in "Genera of Fishes.")

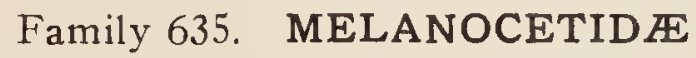

Melanocetus Günther, 1864, $333 . \quad$ Lyocetus Günther, 1887, 437.

\section{Family 636. CAULOPHRYNID在}

Caulophryne Goode \& Bean, 1895, 468.

\section{Family 637. ACERATIID $\mathbb{E}$}

Aceratias Brauer, 1902, 497.

Haplophryne Regan, 1912, 545.

Family 638. GIGACTINID瓜

Gigantactis Brauer, 1902, 497. 



\section{INDEX A}

\section{Generic Names Not Included in "Genera of Fishes."}

In each of the four parts of the work entitled "Genera of Fishes," is an index of the names included, namely: in Part 1, 1758 to 1833 ; in Part 2, 1833 to 1858; in Part 3,1859 to 1880 ; in Part 4, 1881 to 1920 . The present list supplements these, including names overlooked or published since 1920 .

Acanthemblemaria, 233
Acrorhabdus, 112
Actinochromis, 218
Acanthenchelys, 133
Agonus, 215
Aleposomus, 122
Alisodon, 144
Allomycterus, 241
Allosomus, 124
Alticus, 232
Amphotistius, 104
Auchenipterus, 146
Ancistrodon, 239
Anodontoglanis', 147
Antacanthus, 93
Antimora, 164
Aprolepis, 226
Archæonectes, 110
Arnoldites, 164
Asymmetron, 81
Atractophor'us, 245
Atule, 185
Aulonocara, 220
Aurigequula, 186
Austroglossus, 170
Axelia, 109
Bathygadus, 162
Bathymacrops, 245
Bertoniolus, 137
Birgeria, 112
Blavania ${ }^{2}, 145$
Blennophis, 232

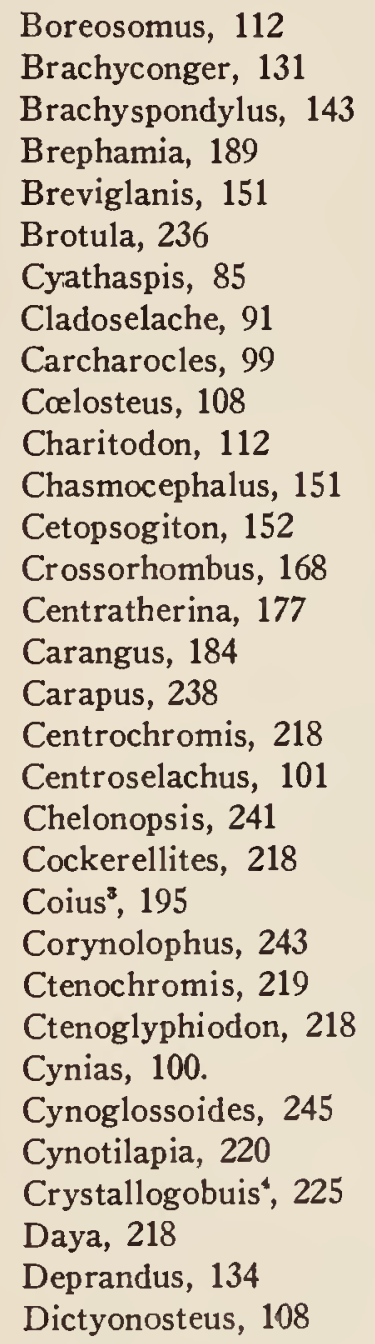

Diplocercides, 109
Drepanichthys, 205
Electrona, 155
Endeconger, 131
Enantcopus', 220
Eosemionotus, 114
Eulepidorhamphus, 161
Eumecichthys, 166
Eumegistus, 181
Eusolea, 170
Evermannichthys, 226
Exoglossops, 144
Exolissus, 217
Gargariscus, 216
Gargilius, 164
Georgichthys, 144
Glaucolepis, 112
Glyptauchen, 210
Grypodon, 239
Gyrace, 100
Hemicaranx, 185
Heminodus, 216
Heterochromis, 220
Heteronarce, 104
Hinalea, 223
Histioclinus, 232
Histiopterus, 199
Holodus, 97
Hololepis, 187
Homea, 82
Hoplochromis, 218
Hoplodus, 94

${ }^{1}$ Anodontoglanis Rendahl, "Fish, N. W. Australia." Zool. Mus. Kristiania, 1922, 168 ; orthotype $A$. dahli Rendahl.

'Blavania Hora, Rec. Ind. Mus., 1920, 202; orthotype B. annandalei Hora.

${ }^{8}$ Coius Hamilton, "Genera of Fishes," 1822, 172.

${ }^{4}$ Crystallogobius (Latrunculodes) and Boreogobius (Latrunculus, preoccupied), are minute, transparent, annual gobies. Aphia, with forked caudal fin, is an atherine, not a goby.

'Enantiopus Boulenger, loc. cit., 1906; orthotype E. melanogenys Boul. 
Hyomacrurus ${ }^{\circ}, 163$

Hyphalonedrus, 154

Ioscion, 202

Ischnacanthus, 91

Laguvia, 148

Lebistes, 158

Leioderma, 109

Leptochilichthys, 122

Lestichthys, 154

Lethrinops, 220

Lissocampus, 175

Loa, 207

Longirostrum, 185

Lumpenus, 235

Leucochlamys, 237

Lyconodes, 245

Maccullochina, 189

Macronoides, 148

Macropleurodus ${ }^{7}, 220$

Mænioides, 197

Malapterus, 221

Margrethia, 127

Melanorhinus, 178

Meridensia, 112

Metzelaaria, 209

Microrasbora, 144

Micropercops, 226

Mylacanthus, 109

Neocyttus, 171

Neoclinus, 231

Neoscombrops, 245

Niphon, 191

Notidanion, 97

Notogrammus, 234

Nomorhamphus ${ }^{8}, 161$

Nyctimaster, 155

Omochelys, 133
Onychopristis, 102

Oroptychus, 95

Ovoides, 240

Oxytomodus, 96

Pachythrissops, 117

Parabrotula, 237

Paracitharus, 168

Parachromis, 220

Paradistichodus, 138

Paralepidotus, 112

Paraphyllodus, 221

Paraplotosus, 147

Parasphenanchias, 245.

Pareutropius, 149

Paropsis, 184

- Peleunominus, 245

Percanthias, 194

Perleidus, 112

Petalichthys, 161

Phenacobrycon, 137

Phractolæmus, 123

Physodon, 100

Phytichthys, 234

Platotichthys, 168

Platybelone, 161

Platysoma, 190

Prionopleurus, 114

Psammodus, 93

Pseudepapterus ${ }^{\circ}, 146$

Pseudocitharichthyes, 168

Pseudodax, 221

Pseudotropheus, 220

Psilichthys, 111

Pterophrynoides, 242

Pterygiocottus, 212

Pungitius, 174

Radcliffella, 226
Rhabdosebastes, 193

Rhamphochromis, 220

Rhamphodermogenys, 161

Rhechias, 131

Rhomboplitoides, 196

Rhyacanthias $^{10}, 194$

Rouleina, 122

Sambwa, 144

Sassenia, 109

Scæops, 167

Scalicus, 216

Scepterias, 189

Scleracanthus, 109

Scombrolabrax, 189

Scopas, 207

Seriolina, 185

Sigmodus, 108

Simenchelys, 130

Siphonostoma, 175

Sphœroides, 240

Squamicreedia, 230

Sternias, 212

Stichæus, 234

Strongylura, 160

Synanceichthys, 210

Synapter etmus, 154

Stylophthalmordes ${ }^{11}, 127$

Stylorhynchus, 113

Tænioconger, 131

Tekla ${ }^{12}, 232$ (Note 532)

Tifia, 207

Tomodus, 96

Trachinops, 194

Trachypterophrys, 167

Trossulus, 154

Trypauchen, 227

Ulaula, 196

"."Genera of Fishes," p. 570; the name Hyostomus in proof-sheets changed to Hyomacrurus in publication. Hyomacrurus has priority.

"Mucropleurodus Regan, "Cichlid Fishes of Lake Victoria," Proc. Zool. Soc. Lond., 1922, 189; orthotype Paratilapia bicolor Boulenger.

"Nomorhamphus Weber \& de Beaufort, "Indo-Austr. Fishes IV," 1922, 141; orthotype $N$. celebensis W. \& de B.

'Denks. Akad. Wien, 1893, 83; orthotype Auchenipterus hasemani Steind.

${ }^{10}$ Rhyacanthias is apparently a synonym of Symphysansdon.

${ }^{1}$ Sanzo, Mem. Acad. Roma, 1920; orthotype S. lovianca Sanzo (larvæ of some Stomiatid). dachner.

${ }^{22}$ Tekla Nichols, "Copeia," 1922, 69; orthotype Cremnobates fasciatus Stein- 
Ulcigobius, 226

Ulcina, 215

Verrugato, 195

Vespicula, 209

Vesposus, 171
Wimania, 109

Xenocephalidæ, 235

Xenodolamia, 99

Xenogobius, 226

Xenolepidichthys, 245.
Xestias, 179

Xiphypops, 207

Xystrias, 168

Zalieutes, 242

Zelotes, 160

Note.-In the Report of the Fisheries and Marine Biological Survey of South Africa, received as these pages go through the press, are two important papers, the first on "The Heterosomata (Flat Fishes)" by Cecil von Bonde, the other "Deep Sea Fishes, Part I," by J. D. F. Gilchrist. The following new genera are proposed:

Cynoglossoides von Bonde, 1822, 23 ; orthotype Cynoglossus attenuatus Gilchrist (Cynoglosside).

Atractophorus Gilchrist, 48; orthotype A. annectens Gilchrist (Squalide).

Bathymacrops Gilchrist, 53 ; orthotype B. macrolepis G. (Bathylagide).

Pelecinomimus Gilchrist, 56; orthotype P. picklei G. (Cetomimida).

Lyconodes Gilchrist, 59; orthotype .L. argenteus G. (Macrourida).

Neoscombrops Gilchrist, 67; orthotype $N$. annectens G. (Scombropida).

Parasphenanthias Gilchrist, 69 ; orthotype $P$. zueberi G. (Serranide).

Xenolepidichthys Gilchrist, 73; orthotype X. dalgleishi G. (Grammicolepida). 


\section{INDEX B}

Names of Families and Higher Groups Included in the Present Paper.

Abyssocottidæ, 214

Acanthoclinidæ, 194

Acanthoëssidæ, 91

Acanthodei, 91

Acanthodidæ, 91

Acanthopterygii, 376

Acanthuridæ, 207

Acanthuriformes, 207

Aceratiidæ, 243

Achiridæ, 169

Acipenseridx, 113

Acropomatidæ, 189

Acrotidx, 183

Actinistia, 109

Actinopteri, 111

Adiposiidæ, 145

Adrianichthyidæ, 160

Aëtobatidæ, 105

Agonidæ, 215

Agriopidæ, 209

Alabidæ, 129

Alabiformes, 129

Alepisauridæ, 156

Alepocephalidæ, 122

Albulidæ, 118

Albuloidei, 118

Allotriognathi, 165

Alopiidæ, 99

Ambassidæ, 189

Amblycipitidæ, 148

Amblyopsidæ, 160

Ameiuridx, 147

Amiatidæ, 116

Amiidæ, 116

Amiidæ, 188

Amioidei, 115

Ammodytidæ, 230

Ammodytiformes, 230

Amphacanthi, 208

Amphiliidæ, 149

Amphioxi, 81

Amphioxidæ, 81
Amphioxididæ, 81

Amphipnoidæ, 129

Amphisilidæ, 176

Amphistiidæ, 204

Anabantidæ, 176

Anablepidæ, 160

Analithidæ, 103

Anarhichadidæ, 235

Anarrhichthyidæ, 235

- Anaspida, 87

Ancylostylidæ, 119

Anguillavidæ, 130

Anguillidæ, 130

Anguilloidei, 130

Anodontidx, 137

Anomalopidæ, 172

Anoplopomidæ, 210

Anostomidæ, 137

Anotopteridæ, 127

Antacea, 90

Antennariidæ, 242

Antiarcha, 86

Antigoniidæ, 204

Aphareidæ, 196

Aphredoderidæ, 165

Apodes, 129

Apogonidæ, 188

A ploactidæ, 209

Aplochitonidx, 125

Aplodactylidæ, 204

Apolectidæ, 184

Apteronotidæ, 138

Arapaimidæ, 123

Archæomænidæ, 116

Archencheli, 129

Argentinidæ, 125

Argidæ, 152

Ariidæ, 145

Arripidæ ${ }^{13}, 195$

Arthrodira, 88

Arthropteridæ, 103

Arthrothoraci, 88
Ascelichthyidæ, 214

Asineopidæ, 165

Aspredinidæ, 151

Asterodermidæ, 103

Asterodontidæ, 112

Asterosteidæ, 88

Aspidoganoidei, 84

Aspidophoroididæ, 215

Aspidorhynchidæ, 115

Aspidorhini, 85

Astraspidæ, 85

Astrolepidæ, 86

Astronesthidæ, 127

Atelaxia, 166

Ateleaspidæ, 86

Ateleopodidæ, 156

Atherinidæ, 177

Atopoclinidæ, 233

Aulopidæ, 153

Aulorhynchidæ, 174

Aulostomi, 175

Aulostomidæ, 175

Azygostei, 86

Bagridæ, 148

Balistidæ, 239

Banjosidæ, 197

Bathyclupeidæ, 186

Bathylagidæ, 125

Bathymasteriformes, 230

Bathydraconidæ, 228

Bathymasteridx, 230

Bathypteroidæ, 154

Bathythrissidæ, 119

Batoidei, 102

Batrachoididæ, 238

Bedotiidæ, 177

Belonidæ, 160

Belonorhynchidæ, 113

Bembropidæ, 229

Benthosauridæ, 154

Berycidæ, 172

Berycoidei, 172

${ }^{19}$ In words of this type classical authority prefers the shorter form to the less euphonious Arripidida, and the like. (Dr. H. R. Fairclough.) 
Berycopsidæ, 172

Birkeniidæ, 87

Bleekeriidæ, 230

Blenniidæ, 232

Blenniiformes, 231

Blepsiidæ, 212

Blochiidæ, 180

Bothide, 167

Bothrolepidæ, 86

Bovictidæ, 228

Brachionichthyidæ, 242

Brachiopteridæ, 105

Bramidæ, 181

Bramiformes, 181

Branchiostegidæ, 202

Branchiostomidæ, 81

Bregmacerotidæ, 163

Brotulidæ, 236

Brotuliformes, 236

Bunocephalidæ, 151

Calamoichthyidæ, 110

Callichthyidx, 152

Callionymidæ, 229

Callionymiformes, 229

Callipterygidæ, 228

Callorhynchidæ, 107

Callyodontidx, 223

Canthigasteridæ, 241

Capriformes, 204

Caproidæ, 204

Caracanthidæ, 210

Carangidæ, 184

Carangiformes, 183

Carapidæ, 238

Carcharhinidæ, 100

Carchariidæ, 99

Carencheli, 129

Caristiidæ, 173

Cataphracti, 208

Catopteridæ, 112

Catostomidx, 139

Caulophrynidæ, 243

Cebidichthyidæ, 233

Centriscidæ, 176

Centrobates, 104

Cephalacanthidæ, 217

Cephalaspidæ, 86

Cephalopteridæ, 105

Cepolidie, 203

Ceraspidæ, 85
Ceratiidæ, 243

Ceratodontidæ, 110

Cerdalidæ, 235

Cestraciontes, 92

Cestraciontidæ, 95

Cetomimidx, 156

Cetopsidæ, 152

Cetorhinidx, 99

Centrarchidæ, 189

Centrolophidæ, 182

Centropomidæ, 190

Cetunculi, 156

Chacidæ, 149

Chætobontidæ, 206

Chætodontiformes, 206

Chænopsidæ, 233

Champsodontidæ, 231

Chanidæ, 119

Channichthyidx, 228

Characidæ, 134

Characinidæ, 134

Characodontidæ, 159

Chaudhuriidæ, 129

Chauliodontidæ, 127

Chaunacidæ, 242

Cheilodactylidæ, 204

Chelonichthyidæ, 88

Cheirothricidæ, 153

Chiasmodontidæ, 230

Chilodipteridæ, 188

Chimæridæ, 106

Chimaeroidci, 105

Chimarrhichthyidæ, 229

Chirocentridæe, 119

Cirrostomi, 81

Chironemidæ, 204

Chismopnea, 105

Chlamydoselachidæ, 97

Chlorophthalmidæ, 154

Chondrenchelyidæ, 92

Chondrichthyes, 156

Chondropterygii, 90

Chondrostei, 111

Chondrosteidx, 113

Chonerhinidæ, 240

Chromides, 218

Cichlidæ, 219

Cirrhitidæ, 203

Cirrhitoidei, 203

Citharinidæ, 138
Cladistia, 110

Cladodontidæ, 92

Cladoselachea, 91

Cladoselachidæ, 91

Clariidæ, 149

Clinidæ, 231

Clupeidæ, 120

Clupeoidei, 119

Clupisudidæ, 123

Cobitidæ, 144

Coccosteidæ, 88

Cochliodontidæ, .94

Colacanthidæ, 109

Colacanthini, 109

Cœlopedidæ, 85

Colobodontidæ, 112

Colocephali, 133

Comephoridæ, 215

Congiopodidæ, 209

Congridæ, 130

Congrogadidx, 235

Congroidei, 130

Coregonidæ, 124

Coridx, 222

Coryphænidæ, 181

Coryphænoididæ, 162

Coryphæniformes, 181

Cottidæ, 213

Cottiformes, 211

Cottocomephoridæ, 215

Creediidæ, 229

Cromeriidæ, 123

Crossognathidæ, 157

Crossopterygii, 108

Cryptacanthodidæ, 235

Ctenodipneusta, 110

Ctenodipterini, 110

Ctenodontidæ, 110

Ctenothrissidæ, 173

Curimatidæ, 137

Cycliæ, 87

Cycloganoidei, 115

Cyclogasteridæ, 216

Cyclopteridæ, 215

Cyclopteriformes, 215

Cýclostomi, 82

Cyemidæ, 132

Cynoglossidx, 171

Cyprinidæ, 139

Cyprinodontes, 157 
Cyprinodontidæ, 158

Dactylopteridæ, 217

Dactylopteriformes, 217

Dactyloscopidæ, 231

Dalatiidæ, 102

Dalliidæ, 156

Dapediidæ, 114

Dasyatidæ, 104

Dasybatidæ, 104

Denticidæ, 198

Dercetidæ, 128

Dercetiformes, 128

Derepodichthyidæ, 236

Derichthyidæ, 129

Deripia, 228

Dermopteri, 82

Dianidæ, 182

Dictyopygidæ, 112

Dinichthyidæ, 88

Dinopterygidæ, 173

Diodontidæ, 241

Diphyodontidæ, 113

Diplacanthidæ, 91

Diplomystidæ, 145

Diploprionidæ, 195

Diplospondyli, 97

Dipneusta, 110

Dipnoi, 110

Dipteridæ, 110

Diretmidæ, 172

Discobatidæ, 103

Discocephali, 227

Doliichthyidæ, 227

Doradidæ, 146

Dorosomidre, 122

Dorypteridæ, 112

Draconettidæ, 229

Drepanaspidæ, 85

Drepanidæ, 205

Dussumieriidæ, 121

Dysommidx, 130

Echelidæ, 131

Echeneidæ, 227

Echinorhinidæ, 102

Edestidæ, 96

Elasmobranchii, 90

Elassomidæ, 190

Eleotridæ, 224

Elephenoridæ, 182

Electrophoridæ, 138
Elipesuridæ, 104

Elopidæ, 117

Elopoidea, 117

Embiotocidæ, 217

Emblemariidæ, 233

Emmelichthyidæ, 200

Encheliidæ, 131

Enchelycephali, 130

Enchodontidx, 126

Enchodontoidei, 126

Engraulidæ, 122

Enoplosidæ, 199

Epelasmia, 206

Ephippidæ, 205

Ephippiformes, 205

- Epigonichthyidæ, 81

Epinephelidæ, 191

Eptatretidæ, 82

Erilepidæ, 210

Erisichthidæ, 115

Eretmophoridæ, 164

Ereuniidæ, 214

Erythrichthyidæ, 200

Erythrinolepidæ, 119

Esocidæ, 157

Etheostomidæ, 187

Eucentrarchidæ, 189

Eugnathidæ, 116

Euphaneropidæ, 86

Furidæ, 116

Eurypharyngidæ, 134

Euselachii, 97

Eventognathi, 139

Evermanellidæ, 154

Exocœtidæ, 161

Fierasferidæ, 238

Fistulariidæ, 175

Fitzroyiidæ, 160

Flutidæ, 129

Forficidæ, 160

Gadidæ, 163

Gadopsidæ, 204

Gadopsiformes, 204

Gaidropsaridæ, 163

Galaxiidæ, 125

Galei, 97

Galeidæ, 100

Galeoidei, 100

Galeorhinidæ, 100

Ganoidei, 111
Ganolytidæ, 118

Gasteropelecidæ, 137

Gasterosteidæ, 173

Gempylidæ, 180

Geotriidæ, 83

Gerridæ, 200

Gigactindæ, 243

Gigactiniidæ, 243

Giganturidæ, 166

Ginglymodi, 115

Ginglystomidæ, 98

Girellidæ, 199

Glaniostomi, 111

Glaniostomi, 113

Glaucosomidæ, 195

Gnathanacanthidæ, 210

Gobiesocidæ, 238

Gobiidæ, 224

Gobioidea, 224

Gobioididæ, 227

Gonorhynchidæ, 120

Gonostomidæ, 127

Goodeidæ, 159

Grammicolepidæ, 171

Gymnarchidæ, 124

Gymnodontes, 240

Gymnonoti, 138

Gymnotidæ, 138

Gyracanthidæ, 92

Hæmulidæ, 196

Halecomorphi, 115

Halosauridæ, 128

Haplomi, 156

Harpagiferidæ, 228

Harpodontidæ, 154

Helogenidæ, 150

Helostomidæ, 176

Homalopteridæ, 145

Hemerocœetidæ, 229

Hemibranchii, 173

Hemiodontidæ, 137

Hemiramphidæ, 161

Hemiscylliidæ, 97

Hemitripteridæ, 214

Henicichthyidæ, 203

Hepatidæ, 207

Heterenchelyidæ, 133

Heterocerci, 111

Heterocongridæ, 131

Heterodontidæ, 95 
Heterognathi, 134

Heteromi, 128

Heterostraci, 85

Heterosomata, 166

Heterotidæ, 123

Hexagrammidæ, 211

Hexagrammiformes, 210

Hexanchidæ, 97

Himantolophidæ, 243

Hiodontidæ, 120

Hippocampidæ, 174

Hippoglossidæ, 168

Histichthyes, 165

Histiopteridæ, 199

Holconoti, 217

Hoplichthyidæ, 211

Holocentridæ, 173

Holocephali, 105

Holoptychidæ, 108

Holostei, 115

Holostomi, 129

Hoplopagridæ, 195

Hoplopleuri, 128

Hoplopterygidæ, 172

Hybodontidæ, 95

Hyperoartia, 83

Hyperotreta, 82

Hypophthalmidæ, 150

Hypoptychidæ, 230

Hypostomides, 175

Hypotremata, 102

Hysterocarpidæ, 217

Icelidæ, 212

Ichthyodectidæ, 119

Ichthyocephali, 129

Ichthyodorulites, 96

Ichthyodorulites, 107

Ichthyotomi, 91

Icosteidæ, 183

Icosteiformes, 183

Ictinocephalidæ, 91

Idiacanthidæ, 127

Ilyophidæ, 130

Inermiidæ, 199

Iniomi, 153

Ipnopidæ, 155

Ischnacanthidæ, 91

Isopholidæ, 116

Isospondyli, 116

Istiophoridæ, 181
Janassidæ, 93

Janassides, 92

Jordaniidæ, 211

Jugulares, 228

Kneriidæ, 123

Kuhliidæ, 190

Kurtidæ, 186

Kurtiformes, 186

Kyphosidæ, 199

Labracoglossidæ, 203

Labridæ, 221

Labyrinthici, 176

Lactariidæ, 186

Lamnidæ, 99

Lamnoidei, 99

Lampridæ, 166

Lasaniidæ, 87

Latidæ, 191

Latilidæ, 202

Latridæ, 204

Leiognathidæ, 186

Lepidoglanidæ, 149

Lepidopidæ, 180

Lepidosirenidæ, 111

Lepidostei, 115

Lepisosteidæ, 115

Lepidotidæ, 114

Leptocardii, 81

Leptocephalidæ, 130

Leptolepidæ, 117

Leptoscopidæ, 231

Lethrinidæ, 197

Limnichthyidæ, 229

Liodesmidæ, 116

Liparidæ, 216

Liparopidæ, 215

Lipogenyidæ, 128

Lobotidæ, 195

Lophiidæ, 242

Lophobranchii, 174

Lophotidæ, 166

Loricariidæ, 152

Loricati, 208

Luciidæ, 157

Luciocephalidæ, 176

Lumpenidæ, 235

Lutianidæ, 195

Luvaridæ, 181

Luvariformes, 181

Lycodapodidæ, 236
Lycodidæ, 236

Lyomeri, 111

Lyomeri, 134

Lyopomi, 128

Lysopteri, 111

Macristiidæ, 123

Macropetalichthyidæ, 88

Macrosemiidæ, 115

Macrorhamphosidæ, 176

Macrouridæ, 162

Macrouroididæ, 162

Macruridæ, 162

Mænidæ, 200

Malacanthidæ, 202

Malacopterygii, 116

Malacosteidæ, 155

Malapteruridæ, 150

Malthidæ, 242

Mantidæ, 105

Marsipobranchii, 82

Mastacembelidæ, 129

Masticura, 104

Maurolicidæ, 127

Medidæ, 144

Megalichthyidæ, 108

Megalopidæ, 118

Melamphaidæ, 172

Melanocetidæ, 243

Melanotæniidæ, 177

Menaspidæ, 86

Menidæ, 185

Merlucciidæ, 164

Merolepidæ, 200

Microcyprini, 157

Microdesmidæ, 235

Microstomidæ, 125

Micropteridæ, 189

Mitsukurinidæ, 99

Mobulidæ, 105

Mochokidæ, 150

Molidx, 241

Monacanthidæ, 239

Monocentridæ, 172

Monodactylidæ, 205

Monopteridæ, 129

Mordaciidæ, 83

Moringuidæ, 133

Mugiloididæ, 229

Mormyridæ, 124

Moronidæ, 191 
Mugilidæ, 178

Mullidæ, 200

Murænesocidæ, 130

Murænidæ, 133

Murænolepidæ, 164

Myctophidæ, 155

Myelozoa, 81

Myliobatidæ, 105

Mylostomidæ, 89

Myriacanthidæ, 106

Myridæ, 131

Myrocongridæ, 130

Myxinidæ, 82

Myzontes, 82

Nandidæ, 202

Nannatherinidæ, 177

Narcaciontes, 103

Narcaciontidæ, 103

Narcobatidæ, 103

Neenchelyidæ, 133

Nematistiidæ, 185

Nematognathii, 145

Nemichthyidæ, 132

Nemipteridæe, 198

Neolabridæ, 223

Neolethrinidæ, 197

Neophrynichthyidæ, 214

Nettastomidæ, 131

Niphonidæ, 191

Nomeidæ, 183

Notacanthidæ, 128

Notidani, 97

Notogoneidæ, 120

Notograptidæ, 232

Notopteridæ, 123

Notopteroidei, 123

Nototheniidæ, 228

Nototheniiformes, 228

Odacidæ, 223

Odontaspidæ, 99

Odontodontidæ, 86

Odontostomidæ, 154

Ogcocephalidæ, 242

Oligopleuridæ, 117

Oligoridæ, 191

Omosudidæ, 155

Onchidx, 92

Oncocephalidæ, 242

Onychodontidæ, 109

Ophicephalidæ, 176
Ophichthyidæ, 132

Ophidiidæ, 237

Ophioclinidæ, 232

Ophiodontidæ, 211

Opistharthri, 97

Opisthognathidæ, 230

Opisthomi, 129

Opisthomyzonidx, 227

Opisthoproctidæ, 127

Oplegnathidæ, 200

Orectolobidæ, 98

Orestiidæ, 158

Orodontidæ, 95

Orthacanthidæ, 91

Osmeridæ, 125

- Osphronemidæ, 176

Ostariophysi, 134

Osteoglossidæ, 123

Osteoglossoidea, 123

Osteolepidæ, 109

Osteostraci, 86

Ostraciidæ, 240

Ostracodermi, 240

Ostracodermi, 84

Ostracodermi, 240

Ostracophori, 84

Otolithidæ, 202

Oxudercidx, 230

Oxylebiidæ, 211

Oxynotidæ, 102

Owstoniidæ, 231

Pachycormidæ, 115

Pachyrhizodontidæ, 118

Palæoniscidæ, 111

Palæorhynchidæ, 180

Palæspondylidæe, 87

Pampidæ, 182

Pangasiidæ, 149

Pantodontidæ, 123

Paralepidæ, 154

Paralichthodidæ, 169

Paralichthyidæ, 168

Parapercidæ, 229

Pareioplitæ, 208

Patæcidæ, 210

Pediculati, 242

Pegasidæ, 175

Pelecopteridæ, 115

Pempheridæ, 194

Pentanchidæ, 98
Percesoces, 177

Percichthyidæ, 188

Percidæ, 186

Perciliidæ, 188

Percomorphi, 177

Percophididæ, 229

Percopsidæ, 165

Periophthalmidæ, 227

Peripristidæ, 94

Peristediidæ, 216

Peronedyidæ, 232

Petalodontidæ, 93

Petromyzonidæ, 83

Phallostethidæ, 160

Phaneropleuridæ, 110

Pharyngognathi, 221

Pharyngopilidæ, 221

Pholidæ, 234

Pholidophoridæ, 116

Pholidophoroidei, 116

Phractolæmidæ, 123

Phth inobranchii, 173

Phyllodontidæ, 221

Phyllolepidæ, 89

Physostomi, 116

Pimelodidx, 150

Pinguipedidæ, 229

Pisces, 108

Placodermi, 86

Placoganoidei, 86

Plagiostomata, 90

Plagyodont idx, 156

Platacidæe, 205

Platycephalidæ, 211

Platycephaliformes, 211

Platypteridæ, 224

Platyrhinidæ, 103

Platysomidæ, 112

Plecoglossidæ, 124

Plectognathi, 239

Plectospondyli, 134

Plesiopidæ, 194

Plethodontidæ, 119

Pleuracanthidæ, 91

Pleuronectidæ, 169

Pleuropterygii, 91

Pliotremidæ, 101

Plotosidæe, 147

Podopterygia, 111

Poecilidæ, 159 
Polyacanthidæ, 176

Polycentridx, 202

Polymixiidæ, 172

Polynemidæ, 178

Polyodontidæ, 113

Polypteridæ, 110

Polyspondyli, 92

Pomacentridæ, 218

Pomadasidæ, 196

Pomatomidæ, 186

Potamotrygonidæ, 104

Priacanthidæ, 194

Priscacaridææ, 218

Pristiophoridæ, 101

Pristidæ, 102

Pristipomidæ, 196

Pristodontidæ, 94

Pronotacanthidæ, 128

Prosarthri, 95

Protocephali, 84

Protopteridx, 111

Protoselachii, 97

Protosphyrænidæ, 115

Protospondyli, 115

Protosyngnathidæ, 174

Psammichthyidæ, 227

Psammodontidæ, 93

Psammosteidæ, 85

Psenidæ, 183

Psettodidæ, 167

Pseudochromidæ, 194

Pseudoplesiopidæ, 194

Pseudotriakidæ, 98

Psilocephalidæ, 240

Psychrolutidæ, 214

Pteraclidæ, 182

Pteraspidæ, 85

Pterichthyidæ, 86

Pternodonta, 97

Pteropsaridæ, 229

Pterothrissidæ, 119

Ptilichthyidæ, 235

Ptychodontidæ, 105

Ptyctodontidæ, 105

Pycnodonti, 114

Pycnodontidæ, 114

Pygæidæ, 208

Pygidiidæ, 151

Rachycentridæ, 186

Rajidæ, 103
Raphiosauridæ, 118

Regalecidæ, 166

Retropinnidæ, 125

Rhamphichthyidæ, 138

Rhamphobatidæ, 102

Rhamphocottidx, 214

Rhamphosidæ, 175

Rhegmatidæ, 194

Rhegnopteri, 178

Rhinellidæ, 128

Rhineodontidæ, 99

Rhinidæ, 102

Rhinobatidæ, 103

Rhinochimeridæ, 107

Rhinopteridæ, 105

Rhipidistia, 108

Rhizodontidæ, 108

Rhodichthyidæ, 237

Rhombodipteridæ, 109

Rhombosoleidæ, 169

Rhyacichthyidæ, 224

Rhynchobatidx, 102

Rogeniidæ, 160

Rondeletiidæ, 156

Runulidæ, 233

Saccopharyngidæ, 134

Salangidæ, 125

Salmonidæ, 124

Salmonoidei, 124

Salmopercæ, 164

Samaridæ, 169

Sarcura, 102

Saurodontidæ, 120

Sauromurænesocidæ, 130

Scarichthyidæ, 223

Scaridæ, 223

Scatophagidæ, 206

Schibeidæ, 149

Sciænidæ, 201

Sclerodermi, 239

Sclerogeni, 208

Scomberesocidæ, 161

Scombridæ, 179

Scombriformes, 179

Scombropidæ, 189

Scopelarchidæ, 154

Scopelidæ, 155

Scorpænichthyidæ, 212

Scorpænidæ, 208

Scorpæniformes, 208
Scorpidæ, 205

Scrotidæ, 112

Scyllioidei, 97

Scylliorhinidæ, 98

Scymnorhinidæ, 102

Scyphophori, 124

Scytalinidæ, 235

Selachii, 90

Selachophidichthyoidei, 97

Selachostomi, 113

Selenichthyes, 166

Selenosteidæ, 89

Semionotidæ, 114

Semiophoridæ, 166

Serranidæ, 193

Siganidæ, 208

Sillaginidæ, 202

Siluridæ, 147

Simenchelyidæ, 130

Siphonognathidæ, 223

Sirenoidei, 110

Sisoridæ, 148

Soleidæ, 169

Solenostomidæ, 174

Somniosidæ, 102

Sparidæ, 197

Sparisomidæ, 223

Sphœrodontidæ, 114

Sphyrænidæ, 178

Sphyrnidæ, 101

Spinacanthidæ, 239

Squalidæ, 101

Squaloidei, 101

Squaloraiidæ, 105

Squamipennes, 206

Squatinidæ, 102

Squatinoidei, 102

Stegophthalami, 88

Steinegeriidæ, 182

Stephanoberycidæ, 171

Sternarchidæ, 138

Sternopthychidæ, 127

Stichæidæ, 234

Stomiatidæ, 126

Stomiatioidei, 126

Stratodontidæ, 128

Stromateidæ, 182

Stromateiformes, 182

Stylephoridæ, 166

Stylodontidx, 114 
Stylophthalmidæ, 127

Sudidæ, 154

Symbranchia, 128

Synancejidæ, 210

Synaphobranchidæ, 130

Synapturidæ, 170

Synbranchidæ, 129

Synchiridæ, 214

Synentognathi, 160

Syngnathidæ, 174

Synodontidæ, 153

Tachysuridæ, 145

Tæniosomi, 166

Tamiobatidæ, 95

Tarrasiidæ, 109

Taxistia, 108

Tectospondyli, 101

Teleostei, 116

Temnothoraci, 88

Tetragonuridæ, 183

Tetraodontidæ, 240

Teuthidæ, 208

Teuthididæ, 207

Thelodontidx, 85

Theraponidx, 197

Thoracostei, 173

Thryptodontidæ, 119

Thunnidæ, 179

Thyestidæ, 86
Thymallidæ, 125

Titanichthyidæ, 89

Tomognathidæ, 126

Torpedinidæ, 103

Torpedinidæ, 150

Toxotidæ, 206

Toxotiformes, 206

Trachichthyidæ, 173

Trachinidæ, 228

Trachipteridæ, 166

Tremataspidæ, 86

Triacanthidæ, 239

Trichiuridæ, 180

Trichodontidæ, 203

Trichodontiformes, 203

Trichonotidæ, 230

Triglidæ, 217

Trigliformes, 216

Triodontidæ, 240

Trygonidx, 104

Trypauchenidæ, 227

Tropidichthyidæ, 241

Umbridæ, 157

Uranoscopidæ, 231

Uranoscopiformes, 230

Urenchelyidæ, 131

Uronemidæ, 110

Urosphenidæ, 175
Veliferidæ, 165

Verilidæ, 196

Xenacanthidx, 91

Xenarchi, 165

Xenesthidæ, 160

Xenichthyidæ, 195

Xenoberyces, 171

Xenocephalidæ, 235

Xenocongridæ, 130

Xenomi, 156

Xenopteri, 238

Xenopterygii, 238

Xiphasiidæe, 235

Xiphidiontidæ, 234

Xiphiidæ, 181

Xiphiiformes, 180

Xiphiorhynchidæ, 181

Xiphisteridæ, 234

Xiphostomidæ, 137

Xystæmidæ, 200

Zanclidæ, 207

Zancliformes, 207

Zaniolepidæ, 211

Zaproridæ, 230

Zeidæ, 171

Zeoidei, 171

Zoarcidæ, 236

Zoaricformes, 235 





\section{Date Due}

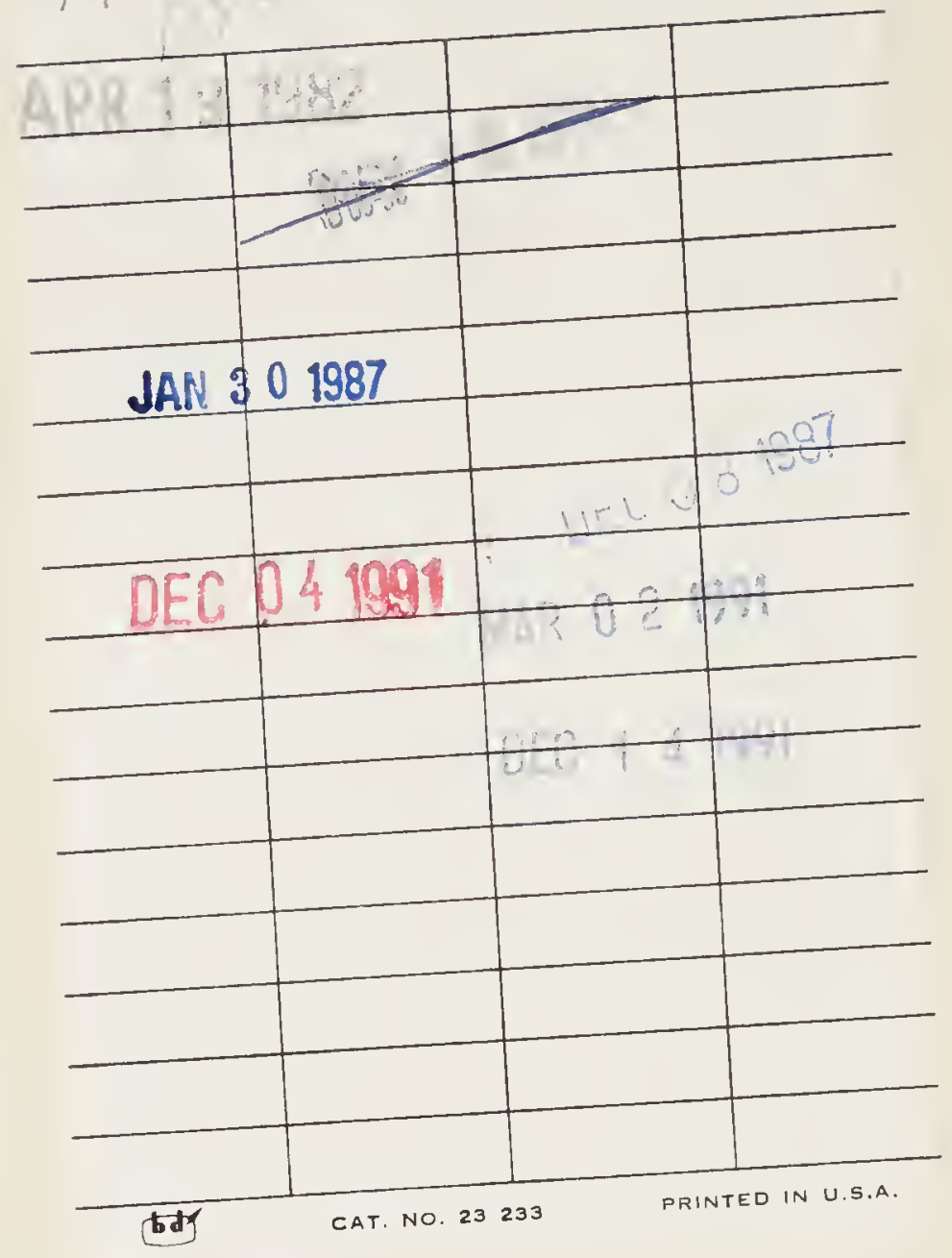




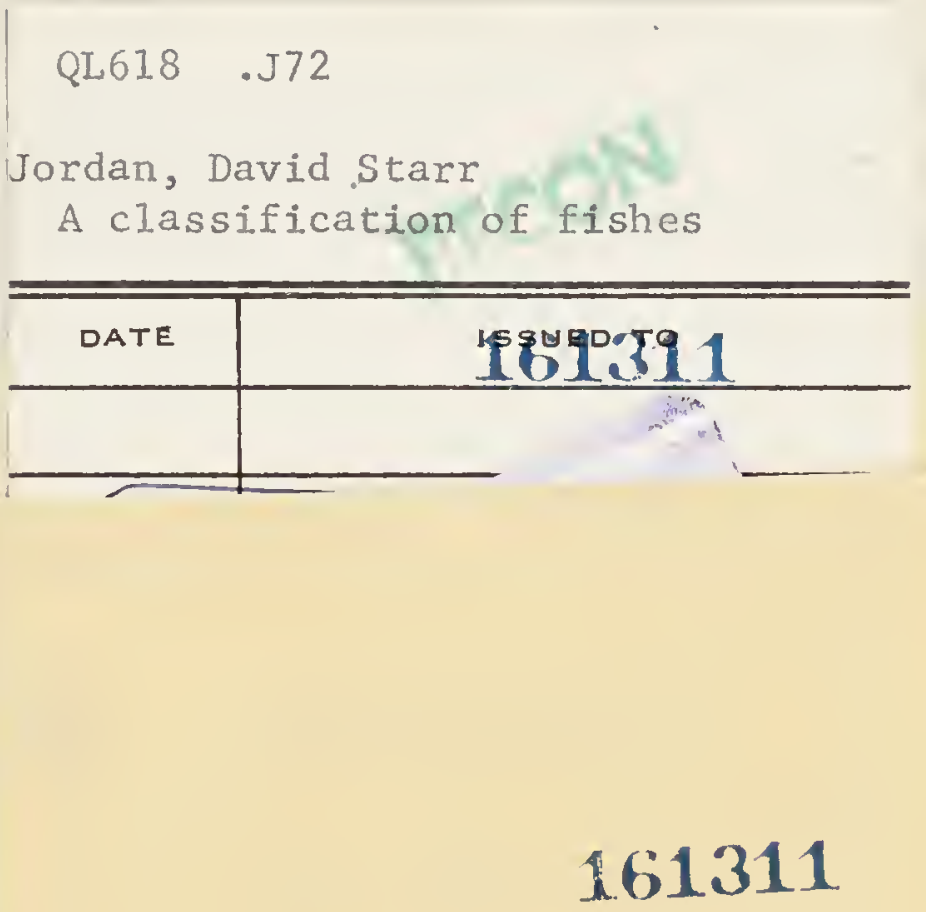


